\title{
Proceedings of the Oak Ridge Electron Linear Accelerator (ORELA) Workshop
}

February 2006

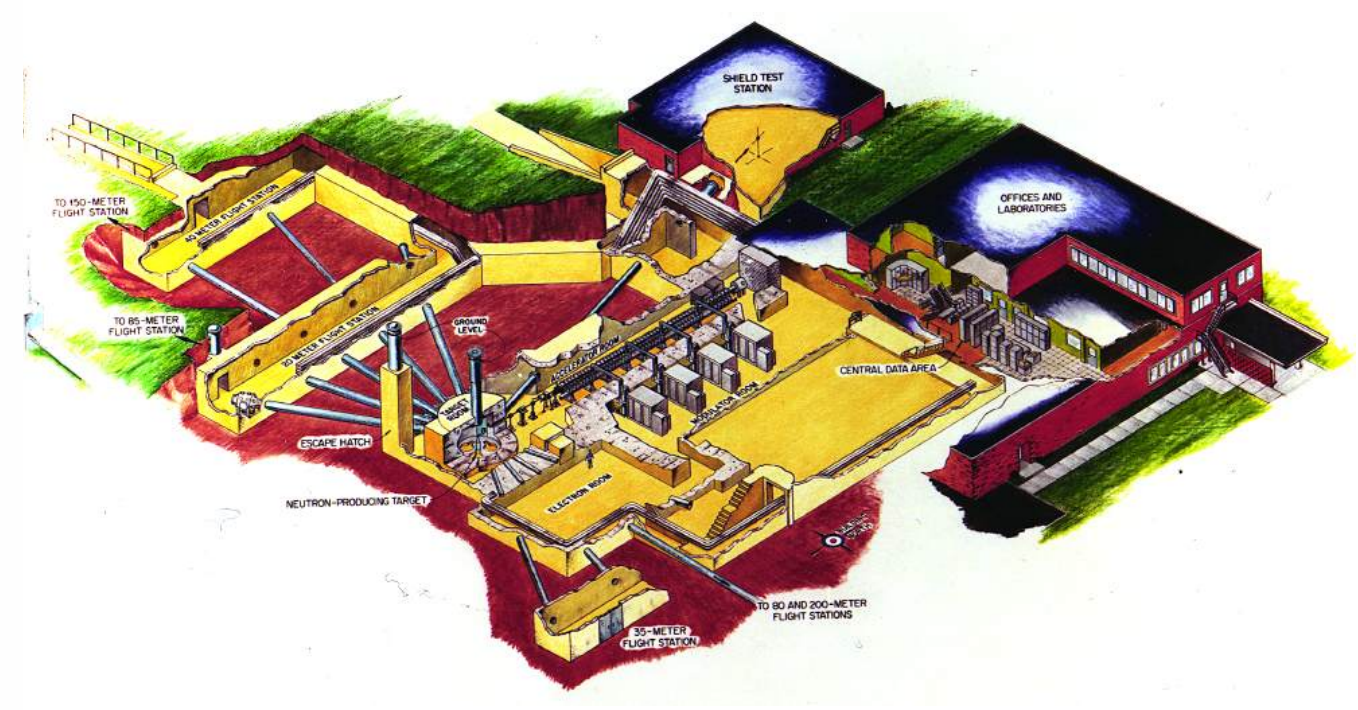

Held at

Oak Ridge National Laboratory

The Conference Center

Oak Ridge, Tennessee

July 14-15, 2005

Sponsored by:
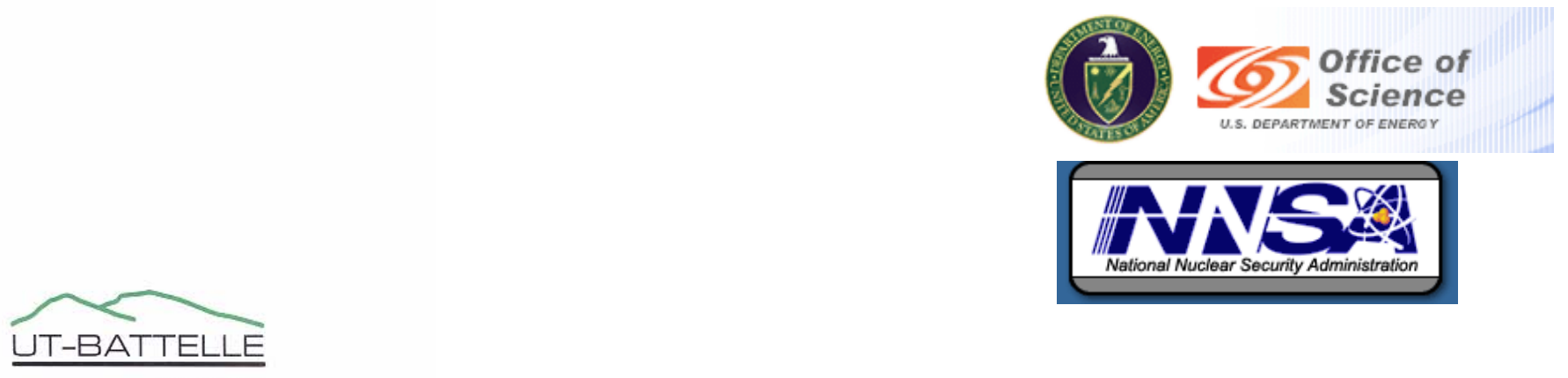


\section{DOCUMENT AVAILABILITY}

Reports produced after January 1, 1996, are generally available free via the U.S. Department of Energy (DOE) Information Bridge:

Web site: http://www.osti.gov/bridge

Reports produced before January 1, 1996, may be purchased by members of the public from the following source:

National Technical Information Service

5285 Port Royal Road

Springfield, VA 22161

Telephone: 703-605-6000 (1-800-553-6847)

TDD: 703-487-4639

Fax: 703-605-6900

E-mail: info@ ntis.gov

Web site: http://www.ntis.gov/support/ordernowabout.htm

Reports are available to DOE employees, DOE contractors, Energy Technology Data Exchange (ETDE) representatives, and International Nuclear Information System (INIS) representatives from the following source:

Office of Scientific and Technical Information

P.O. Box 62

Oak Ridge, TN 37831

Telephone: 865-576-8401

Fax: $865-576-5728$

E-mail: reports@osti.gov

Web site: http://www.osti.gov/contact.html

This report was prepared as an account of work sponsored by an agency of the United States Government. Neither the United States government nor any agency thereof, nor any of their employees, makes any warranty, express or implied, or assumes any legal liability or responsibility for the accuracy, completeness, or usefulness of any information, apparatus, product, or process disclosed, or represents that its use would not infringe privately owned rights. Reference herein to any specific commercial product, process, or service by trade name, trademark, manufacturer, or otherwise, does not necessarily constitute or imply its endorsement, recommendation, or favoring by the United States Government or any agency thereof. The views and opinions of authors expressed herein do not necessarily state or reflect those of the United States Government or any agency thereof. 
PROCEEDINGS OF THE

\title{
OAK RIDGE ELECTRON LINEAR ACCELERATOR (ORELA) WORKSHOP
}

\author{
Held at \\ Oak Ridge National Laboratory \\ Oak Ridge, Tennessee 37831, USA \\ July 14-15, 2005
}

Date Published: February 2006

Compiled by: Michael E. Dunn, Meeting Coordinator

\section{Contributing Authors:}

M. E. Dunn (ORNL)

Y. Danon (RPI)

F. S. Dietrich (LLNL)

P. Finck (ANL)
K. H. Guber (ORNL)

R. Haight (LANL)

A. K. Kerman (MIT)

P. E. Koehler (ORNL)
R. C. Little (LANL)

J. N. McKamy (NNSA)

G. E. Mitchell (NCSU)

M. G. Mustafa (LLNL)
D. W. Nigg (INL)

P. Oblozinsky (BNL)

J. E. Rushton (ORNL)

T. E. Valentine (ORNL)

Prepared by the

OAK RIDGE NATIONAL LABORATORY

P.O. Box 2008

Oak Ridge, Tennessee 37831-6285

managed by

UT-Battelle, LLC

for the

U.S. DEPARTMENT OF ENERGY

under contract DE-AC05-00OR22725 



\section{Proceedings of the \\ Oak Ridge Electron Linear Accelerator (ORELA) Workshop}

July 14-15, 2005

\section{CONTENTS}

Page

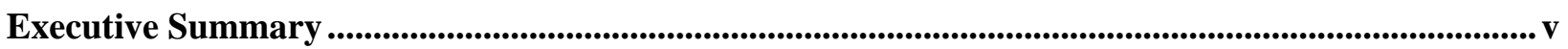

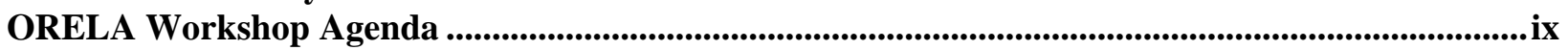

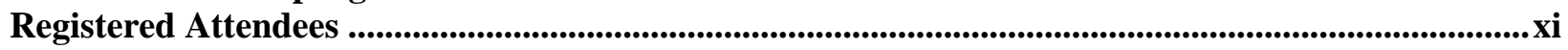

ORELA Workshop Organizing Committee ............................................................................................................xvii

Nuclear Data Needs Supporting Gen-IV Applications-INL Perspective and Initiatives ..................... 1

D. W. Nigg, J. D. Baker, C. A. McGrath, C. L. Riddle, A. Caffrey, J. Anderson,

J. D. Cole, M. W. Drigert, R. Aryaeinejad, Idaho National Laboratory;

R. V. F. Janssens, B. J. Micklich, Argonne National Laboratory;

G. Ter-akopian, Joint Institute for Nuclear Research, Dubna, Russia

NNDC Perspective on Nuclear Reaction Databases and ORELA Pavel Oblozinsky, National Nuclear Data Center, Brookhaven National Laboratory

Oak Ridge National Laboratory Neutron Sciences Overview

Paul E. Koehler, Physics Division, Oak Ridge National Laboratory

Neutron Cross-Section Measurements at ORELA

K. H. Guber, L. C. Leal, R. O. Sayer, P. E. Koehler, H. Derrien, D. Wiarda, and J. A. Harvey

Oak Ridge National Laboratory

LANSCE and ORELA Complementary Measurement Capabilities

Robert C. Haight, Los Alamos Neutron Science Center, Los Alamos National Laboratory

The Gaerttner LINAC Laboratory Review and Current Activity

Y. Danon, ${ }^{,}$R. C. Block, J. A. Burke, ${ }^{,}$N. J. Drindak, ${ }^{,} J$. G. Hoole, ${ }^{j}$ G. Leinweber,

Michel Rapp, ${ }^{a}$ Frank Saglime ${ }^{a}$

${ }^{a}$ Department of Mechanical Aerospace and Nuclear Engineering,

Rensselaer Polytechnic Institute, Troy, NY

${ }^{b}$ KAPL Inc., Lockheed Martin Corporation, Schenectady, NY

Importance of ORELA for Developing Nuclear Reaction Models

F. S. Dietrich, Lawrence Livermore National Laboratory

Nuclear Cross Sections for Stockpile Stewardship Applications at Livermore

M. G. Mustafa, AX Division, Lawrence Livermore National Laboratory

New Opportunities and Synergies for ORELA.

G. E. Mitchell, Department of Physics, North Carolina State University,

Triangle Universities Nuclear Laboratory 


\section{CONTENTS (continued)}

Page

Panel Discussion Minutes_Future Direction and Activities ...................................................................69

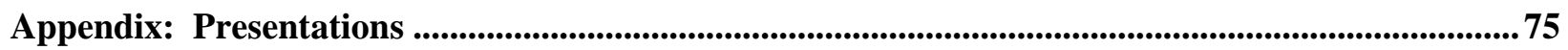




\section{Executive Summary}

The Oak Ridge National Laboratory (ORNL) organized a workshop at ORNL July 14-15, 2005, to highlight the unique measurement capabilities of the Oak Ridge Electron Linear Accelerator (ORELA) facility and to emphasize the important role of ORELA for performing differential cross-section measurements in the low-energy resonance region that is important for nuclear applications such as nuclear criticality safety, nuclear reactor and fuel cycle analysis, stockpile stewardship, weapons research, medical diagnosis, and nuclear astrophysics. The ORELA workshop (hereafter referred to as the Workshop) provided the opportunity to exchange ideas and information pertaining to nuclear crosssection measurements and their importance for nuclear applications from a variety of perspectives throughout the U.S. Department of Energy (DOE). Approximately 50 people, representing DOE, universities, and seven U.S. national laboratories, attended the Workshop. The objective of the Workshop was to emphasize the technical community endorsement for ORELA in meeting nuclear data challenges in the years to come. The Workshop further emphasized the need for a better understanding of the gaps in basic differential nuclear measurements and identified the efforts needed to return ORELA to a reliable functional measurement facility.

To accomplish the Workshop objective, nuclear data experts from national laboratories and universities were invited to provide talks emphasizing the unique and vital role of the ORELA facility for addressing nuclear data needs. The list of invited speakers with information pertaining to the presentation titles is provided in the Workshop Agenda. The first day of the workshop was largely devoted to talks from the invited speakers, and the presentation slides are provided in the Appendix. Nine speakers prepared full papers to complement the presentations in the Appendix, and the contributed papers are compiled in the subsequent sections of the proceedings. At the conclusion of the first day, a dinner meeting was held at the Tennessee Grill in Knoxville, Tennessee, and Jim Rushton, who is director of the ORNL Nuclear Science and Technology Division (NSTD), provided the keynote address, highlighting the "Future of Nuclear Energy in the U.S." The dinner meeting slides are also provided in the Appendix. On the second day, ORNL presented an ORELA facility improvement plan to reestablish reliable ORELA operation for nuclear data measurements. Following the facility improvement discussions, the Workshop participants were given a tour of the ORELA facility by ORNL staff. In order to close out the discussions and draw conclusions from the Workshop, Tim Valentine (NSTD) moderated an expert panel discussion with panelists from Rensselaer Polytechnic Institute (RPI), the Massachusetts Institute of Technology (MIT), the National Nuclear Security Administration (NNSA), Lawrence Livermore National Laboratory (LLNL), and Idaho National Laboratory (INL). The minutes of the panel discussion are provided in the proceedings.

Based on the presentations, papers, and discussions, the following points emerged from the Workshop:

- ORELA has a long history (30-40 years) of providing detailed cross-section data for supporting nuclear applications. Pavel Oblozinsky, who is the director of the National Nuclear Data Center (NNDC) at Brookhaven National Laboratory (BNL), provided the following statistics concerning ORELA's contribution to nuclear databases:

o Within the international Experimental Nuclear Reaction Database (EXFOR/CSISRS), ORELA has made contributions to 334 papers since 1971 with 10 measurement paper contributions between 2000 and 2005.

o In the ENDF/B-VI Release 8 cross-section database, 77 out of 329 materials are directly attributed to ORNL measurement and evaluation efforts that are based on research at ORELA. 
o Numerous ORELA measurements have been used to support the development of the fifth edition of the Atlas of Neutron Parameters by Mughabghab (NNDC), which will be published in 2006. Specifically, the ORELA data used to support the Atlas of Neutron Parameters include data for structural materials; fission products; light nuclei (e.g., ${ }^{16} \mathrm{O}$, ${ }^{19} \mathrm{~F},{ }^{35,37} \mathrm{Cl}$ ); nuclei in the Lu-Pb region; and heavy nuclei such as ${ }^{232} \mathrm{Th},{ }^{231} \mathrm{~Pa},{ }^{233,238} \mathrm{U}$, ${ }^{237} \mathrm{~Np}$, and ${ }^{241,242 \mathrm{~m}} \mathrm{Am}$.

o ORELA has provided measured data that are essential for nuclear model calculations (e.g., average resonance spacing for nuclei, neutron radiative widths for photon strength functions, neutron strength functions for optical model parameterization).

- Multiple DOE programs continue to need ORELA for basic measured nuclear data. These programs include but are not limited to the DOE Nuclear Criticality Safety Program (NCSP) support for Environmental Management (EM) (e.g., the Savannah River Site-titanium), Radioactive Waste (fission product cross sections for burnup credit), NNSA (Stockpile Stewardship and weapons production), Nuclear Energy (Gen III and IV nuclear reactors) and the DOE Office of Science (SC) (nuclear astrophysics). The sole program sponsor for ORELA is the NCSP, infrastructure (staff and building) support is provided by SC. There is significant collateral benefit to the DOE resulting from NCSP and SC funding of ORELA with the potential to contribute even more to Stockpile Stewardship needs in the future.

- Some of the current nuclear data needs include but are not limited to manganese (NCSP/EM), titanium (NCSP/EM), beryllium (NCSP/NNSA and EM), europium (NNSA-Stockpile Stewardship/Subcriticals) and vanadium (NCSP/NNSA weapons production). Based on information from cross-section evaluators and nuclear theory experts, the nuclear data community has obtained all the information that can be used from existing data measurements, and there remain computational problems with existing data in nuclear models (e.g., several percent biases in $k_{\text {eff }}$ calculations for beryllium benchmarks). Therefore, ORELA is needed to fill the gaps that remain in the existing experimental database.

- Based on presentations from nuclear theory experts, cross-section measurement specialists, and cross-section evaluators, computational models cannot single-handedly address all the data needs for supporting nuclear applications. In the past two or three decades, nuclear theory has not advanced to the point where cross sections can be accurately predicted from first principles. Therefore, ORELA is needed to help bridge the gap between nuclear theory and applications.

- ORELA is the only U.S. facility with the requisite energy resolution and beam intensity in the resonance energy region. The comparable RPI Gaerttner accelerator could serve as a backup facility with limitations (i.e., reduced resolution) at intermediate energies relative to ORELA and lower power (i.e., reduced capability to measure small cross sections or to use very small target samples) capabilities. The Argonne National Laboratory (ANL) Intense Pulsed Neutron Source (IPNS) cannot duplicate or replace ORELA. IPNS provides a complementary capability to that of ORELA, not a duplicative capability. IPNS does not have the resolution in the resonance region to match the ORELA capability. The Los Alamos Neutron Science Center (LANSCE) facility is a complementary capability to ORELA, as outlined in the paper by Bob Haight from Los Alamos National Laboratory (LANL).

ORELA is operated on a full cost-recovery basis with no single sponsor providing complete base funding for the facility. Consequently, different programmatic sponsors benefit by receiving accurate crosssection data measurements at a reduced cost to their respective programs; however, leveraging support for a complex facility such as ORELA has a distinct disadvantage in that the programmatic funds are only 
used to support program-specific measurements. As a result, ORELA has not received base funding to support major upgrades and significant maintenance operations that are essential to keep the facility in a state of readiness over the long term. As a result, ORELA has operated on a "sub-bare-minimum" budget for the past 10 to 15 years, and the facility has not been maintained at a level for continued reliable operation for the long term. During the Workshop, Jerry McKamy (NNSA/NA-117) used a hospital patient metaphor that accurately depicts the facility status. ORELA is currently in the intensive care unit (ICU) on life support, and refurbishment efforts are needed to get the "patient" off life support and out to an ordinary hospital room. McKamy further noted that the DOE NCSP is planning to fund immediate refurbishment tasks (\$1.5 M over three years) to help reestablish reliable ORELA operation (i.e., move ORELA from ICU to an ordinary hospital room). Furthermore, the NCSP will work to identify and carry out the actions needed to discharge ORELA from the "hospital" over the next five to seven years.

In accordance with the Workshop objectives, the technical community publicly endorsed the need for a reliable ORELA facility that can meet current and future nuclear data needs. These Workshop proceedings provide the formal documentation of the technical community endorsement for ORELA. Furthermore, the proceedings highlight the past and current contributions that ORELA has made to the nuclear industry. The Workshop further emphasized the operational and funding problems that currently plague the facility, thereby limiting ORELA's operational reliability. Despite the recent operational problems, ORELA is a uniquely capable measurement facility that must be part of the overall U.S. nuclear data measurement portfolio in order to support current and emerging nuclear applications. The Workshop proceedings further emphasize that ORNL, the technical community, and programmatic sponsors are eager to see ORELA reestablish reliable measurement operation and be readily available to address nuclear data challenges in the United States. 


\section{ORELA Workshop Agenda \\ Oak Ridge National Laboratory}

Building 5200, Room 202A (Tennessee)

July 14-15, 2005

Thursday, July 14, 2005

\begin{tabular}{|c|c|}
\hline 9:00 & Mike Dunn \\
\hline 9:05 & Michelle Buchanan \\
\hline $9: 15$ & Mike Dunn \\
\hline 9:30 & Jerry McKamy \\
\hline \multicolumn{2}{|l|}{$9: 45$} \\
\hline $10: 15$ & Dave Nigg \\
\hline $10: 45$ & Phil Finck \\
\hline $11: 15$ & Pavel Oblozinsky \\
\hline \multicolumn{2}{|l|}{$11: 45$} \\
\hline 1:00 & Paul Koehler \\
\hline $1: 30$ & Klaus Guber \\
\hline $2: 00$ & Bob Haight \\
\hline $2: 30$ & Yaron Danon \\
\hline \multicolumn{2}{|l|}{$3: 00$} \\
\hline $3: 30$ & Bob Little \\
\hline 4:00 & Frank Dietrich \\
\hline $4: 30$ & Mohammed Mustafa \\
\hline $5: 00$ & Gary Mitchell \\
\hline $5: 30$ & \\
\hline $7: 00$ & \\
\hline
\end{tabular}

\section{Presentation}

ORNL Introductory Remarks ORNL Associate Lab Welcome to ORNL Director Physical Sciences Directorate ORNL NNSA

INL ANL BNL

ORNL ORNL LANL RPI

LANL

LLNL

LLNL

TUNL

ORELA Status and Workshop Objective

NNSA Perspective on Nuclear Data for Supporting U.S. Nuclear Criticality Safety Program

Coffee Break

Nuclear Data Needs for Supporting GEN-IV Applications The Future of Nuclear Energy and the Role of Nuclear Data NNDC Perspective on Nuclear Reaction Databases and ORELA

Lunch

ORNL Neutron Sciences Overview

ORELA Overview and Capabilities in Resonance Region LANSCE and ORELA Complementary Measurement Capabilities The Gaerttner LINAC Laboratory Review and Current Activity

Coffee Break

LANL-ORNL Nuclear Data Evaluation Collaborations for Supporting NCSP

Importance of ORELA Measurements for Confirming Nuclear Reaction Models

Nuclear Cross Sections for Stockpile Stewardship Applications at Livermore

New Opportunities and Synergies for ORELA

End First Day

Dinner: Tennessee Grill

(http://www.tennesseegrill.com/TG/index.htm)

Chartered bus to Tennessee Grill from Jameson Inn at 6:30

Social (cash bar) 7:00; Dinner 7:30

Speaker: Jim Rushton, Acting Director Nuclear Science \& Technology Division

"Future of Nuclear Energy in the U.S."

Friday, July 15, 2005

$\begin{array}{ll}\text { 8:30 } & \text { Mike Dunn } \\ \text { 8:45 } & \text { Klaus Guber \& Paul } \\ & \text { Koehler }\end{array}$

ORNL ORNL

ORELA Facility Improvement Plan

ORELA Tour (Building 6010)

Panel Discussion: Future Direction \& Activities Moderator: Tim Valentine (ORNL)

Lunch

End Workshop 


\section{Registered Attendees \\ Oak Ridge Electron Linear Accelerator (ORELA) Workshop \\ July 14-15, 2005}

Arbanas, Goran

OAK RIDGE NATIONAL LABORATORY

PO Box 2008, Bldg. 5700, MS-6171

Oak Ridge, TN 37831-6171 USA

865-574-4984

arbanasg@ornl.gov

Briggs, Joseph Blair

IDAHO NATIONAL LABORATORY

2525 N. Fremont Avenue

Idaho Falls, ID 83415-3860 USA

208-526-7628

j.briggs@inl.gov

Cartegni, Lucia

UNIVERSITA DEGLI STUDI DI MILANO

via Illirico, 18

20133 Milano, Italy

(+39) 0270122958

luciacart@yahoo.it

Carter, Linda

9702 Garden Row Drive

Sugar Land, TX 77478 USA

Student

713-412-5443

carterlm@ornl.gov

Chadwick, Mark B.

LOS ALAMOS NATIONAL LABORATORY

B283, PADNWP, LANL

Los Alamos, NM 87545 USA

505-661-8812

mbchadwick@lanl.gov
Danon, Yaron

RENSSELAER POLYTECHNIC INSTITUTE

110 8th Street

Troy, NY 12180 USA

518-276-4008

danony@rpi.edu

Deason, Jerrad

PRAIRIE VIEW A\&M UNIVERSITY

803 Copeland

Lufkin, TX 76904 USA

936-366-7623

jdeason@ornl.gov

Derrien, Herve

OAK RIDGE NATIONAL LABORATORY

PO Box 2008, Bldg. 5700, MS-6171

Oak Ridge, TN 37831-6171 USA

865-574-7928

derrienh@ornl.gov

Dietrich, Frank

LAWRENCE LIVERMORE NATIONAL

LABORATORY

MS L-414, PO Box 808

Livermore, CA 94550 USA

925-422-4521

dietrich2@llnl.gov

Dunn, Michael Edward

OAK RIDGE NATIONAL LABORATORY

PO Box 2008, Bldg. 5700, MS-6170

Oak Ridge, TN 37831-6170 USA

865-574-5260

dunnme@ornl.gov 
Felty, James R.

SCIENCE APPLICATIONS

INTERNATIONAL CORPORATION

2418 Dickerson Street

Arlington, VA 22207 USA

301-903-5494

james.felty@dp.doe.gov

Ferrer, Rodolfo M.

PENN STATE UNIVERSITY

1013 South Allen Street, \#109

State College, PA 16801 USA

717-421-9404

mf166@psu.edu

Fujita, Edward Kei

ARGONNE NATIONAL LABORATORY

9700 S. Cass Avenue

Argonne, IL 60439 USA

630-252-4866

ekfujita@anl.gov

Garland, Marc Alan

UNIVERSITY OF SOUTH CAROLINA

1136 Autumn Circle

Columbia, SC 29206-4986 USA

803-546-1161

garlandmarc@earthlink.net

Gehin, Jess C.

OAK RIDGE NATIONAL LABORATORY

PO Box 2005, Bldg. 5700, MS-6172

Oak Ridge, TN 37831-6172 USA

865-576-5093

gehinjc@ornl.gov

Gill, Daniel F.

PENN STATE UNIVERSITY

219 Waupelani Drive, Apt. 10

State College, PA 16801 USA

724-699-6334

dfg121@psu.edu
Gouw, Reza Raymond

BECHTEL BETTIS, INC

PO Box 79

West Mifflin, PA 15122 USA

412-476-3962

gouwrr@bettis.gov

Greene, Norman Maurice

OAK RIDGE NATIONAL LABORATORY

PO Box 2008, Bldg. 5700, MS-6171

Oak Ridge, TN 37831-6170 USA

865-574-8712

greenenm@ornl.gov

Guber, Klaus Heinrich

OAK RIDGE NATIONAL LABORATORY

PO Box 2008, Bldg. 6010, MS-6356

Oak Ridge, TN 37831-6356 USA

865-574-6117

guberkh@ornl.gov

Haight, Robert Cameron

LOS ALAMOS NATIONAL LABORATORY MS H855

Los Alamos, NM 87545 USA

505-667-2829

haight@lanl.gov

Harvey, John Arthur

OAK RIDGE NATIONAL LABORATORY

PO Box 2008, Bldg. 6010, MS-6356

Oak Ridge, TN 37831-6356 USA

865-483-1495

harveyjm@icx.net

Heinrichs, David P.

LAWRENCE LIVERMORE NATIONAL LABORATORY

7000 East Avenue,

PO Box 808, L-198

Livermore, CA 94002 USA

925-424-5679

heinrichs1@llnl.gov 
Herman, Michal Wladyslaw

BROOKHAVEN NATIONAL LABORATORY

Bldg. 197D

Upton, NY 11973 USA

631-344-2802

mwherman@bnl.gov

Hoole, Jeffrey G.

KNOLLS ATOMIC POWER LABORATORY

PO Box 1072

Schenectady, NY 12301 USA

518-395-6979

hoole@kapl.gov

Ingersoll, Daniel T.

OAK RIDGE NATIONAL LABORATORY

PO Box 2008, Bldg. 5700, MS-6162

Oak Ridge, TN 37831-6162 USA

865-574-6102

ingersolldt@ornl.gov

Kahler, Albert Comstock III

BECHTEL BETTIS, INC

PO Box 79

West Mifflin, PA 15122 USA

412-476-5399

kahlerac@bettis.gov

Kawano, Toshihiko

LOS ALAMOS NATIONAL LABORATORY

MS B283, Theoretical Division

Los Alamos, NM 87545 USA

505-664-0513

kawano@lanl.gov

Kerman, Arthur K.

MASSACHUSETTS INSTITUTE OF TECHNOLOGY

5 Cambridge Center, 4th Floor

Cambridge, MA 02142 USA

617-253-7072

kerman@mitlns.mit.edu
King, Susan Michelle

OAK RIDGE NATIONAL LABORATORY

PO Box 2008, Bldg. 5700, MS-6171

Oak Ridge, TN 37831-6171 USA

865-576-8887

kingsm@ornl.gov

Kirk, Bernie

OAK RIDGE NATIONAL LABORATORY

PO Box 2008, Bldg. 5700, MS-6171

Oak Ridge, TN 37831-6171 USA

865-574-6176

kirkbl@ornl.gov

Klingensmith, Jesse J.

PENN STATE UNIVERSITY

1238 S. Pugh

State College, PA 16801 USA

865-241-0741

jxk390@psu.edu

Koehler, Paul E.

OAK RIDGE NATIONAL LABORATORY

PO Box 2008, Bldg. 6010, MS-6356

Oak Ridge, TN 37831-6356 USA

865-482-7714

koehlerpe@ornl.gov

Larson, Nancy

OAK RIDGE NATIONAL LABORATORY

PO Box 2008, Bldg. 5700, MS-6171

Oak Ridge, TN 37831-6171 USA

865-574-4659

LarsonNM@ornl.gov

Leal, Luiz C.

OAK RIDGE NATIONAL LABORATORY

PO Box 2008, Bldg. 5700, MS-6171

Oak Ridge, TN 37831-6171 USA

865-574-5281

leallc@ornl.gov 
Lemaire, Sebastien

LOS ALAMOS NATIONAL LABORATORY

MS B243

Los Alamos, NM 87545 USA

505-667-0174

lemaire@lanl.gov

Li, Kun

UNIVERSITY OF ILLINOIS AT URBANA CHAMPAIGN

502 W. Main St., \#117

Urbana, IL 61801 USA

217-721-3127

kunli@uiuc.edu

Little, Robert C.

LOS ALAMOS NATIONAL LABORATORY

MS F663, PO Box 1663

Los Alamos, NM 87545 USA

505-665-3487

rcl@lanl.gov

Lovett, Henry Ashton

UNIVERSITY OF SOUTH CAROLINA

835 Ohio Street

Columbia, SC 29201 USA

803-479-2415

hallovett@hotmail.com

Lubitz, Cecil Robert

KNOLLS ATOMIC POWER LABORATORY

PO Box 1072

Schenectady, NY 12301-1072 USA

518-395-7103

lubitz@kapl.gov

MacFarlane, Robert Edwin

LOS ALAMOS NATIONAL LABORATORY

MS B243

Los Alamos, NM 87545 USA

505-667-7742

ryxm@lanl.gov
McKamy, Jerry Neal

NATIONAL NUCLEAR SECURITY

ADMINISTRATION

1000 Independence Ave. SW

NA-117, Germantown Bldg.

Washington, DC 20585-1290 USA

301-903-8031

jerry.mckamy@nnsa.doe.gov

McKnight, Richard D.

ARGONNE NATIONAL LABORATORY

9700 S. Cass Avenue

Argonne, IL 60439 USA

630-252-6088

rdmcknight@anl.gov

Mitchell, Gary Earl

NORTH CAROLINA STATE UNIVERSITY

Dept. of Physics, Box 8202

Raleigh, NC 27695 USA

919-660-2638

mitchell@tunl.duke.edu

Mustafa, Mohammed G.

LAWRENCE LIVERMORE NATIONAL LABORATORY

7000 East Avenue

Livermore, CA 94550 USA

925-422-5523

mustafa1@llnl.gov

Nigg, David W.

IDAHO NATIONAL LABORATORY

PO Box 1625

Idaho Falls, ID 83415 USA

208-526-7627

dwn@inel.gov

Oblozinsky, Pavel

BROOKHAVEN NATIONAL LABORATORY

PO Box 5000

Upton, NY 11973 USA

631-344-2814

oblozinsky@bnl.gov 
Parks, Cecil

OAK RIDGE NATIONAL LABORATORY

PO Box 2008, Bldg. 5700, MS-6170

Oak Ridge, TN 37831-6170 USA

865-574-5280

parkscv@ornl.gov

Pritchard, Megan

OAK RIDGE NATIONAL LABORATORY

PO Box 2008, Bldg 7601, MS-6304

Oak Ridge, TN 37831-6304 USA

865-576-7505

pritchardml@ornl.gov

Ross, Rahn H.

WASHINGTON SAFETY MANAGEMENT SOLUTIONS

2131 Centennial Drive

Aiken, SC 29803 USA

803-502-9623

rahn.ross@wsms.com

Rushton, James

OAK RIDGE NATIONAL LABORATORY

PO Box 2008, Bldg. 5700, MS-6152

Oak Ridge, TN 37831-6152 USA

865-576-7000

rushtonje@ornl.gov

Slaybaugh, Rachel Nicole

PENN STATE UNIVERSITY

305 Ghaner Drive

State College, PA 16803 USA

570-850-3385

rslaybaugh@psu.edu

Sutton, Thomas Mitchell

KNOLLS ATOMIC POWER LABORATORY

PO Box 1072, MS-112

Schenectady, NY 12301-1072 USA

518-395-7047

sutton@kapl.gov
Urashkin, Alexander Y.

TEXAS A\&M UNIVERSITY

4110 College Main, Apt. 46

Bryan, TX 77801 USA

979-574-1219

urashkinay@ornl.gov

Valentine, Timothy E.

OAK RIDGE NATIONAL LABORATORY

PO Box 2008, Bldg. 4500N, MS-6248

Oak Ridge, TN 37831-6248 USA

865-574-0715

valentinete@ornl.gov

Wiarda, Dorothea

OAK RIDGE NATIONAL LABORATORY

PO Box 2008, Bldg. 5700, MS-6171

Oak Ridge, TN 37831-6171 USA

865-576-9838

wiardada@ornl.gov

Williams, Mark

OAK RIDGE NATIONAL LABORATORY

PO Box 2008, Bldg. 5700, MS-6170

Oak Ridge, TN 37831-6170 USA

865-576-5565

williamsml@ornl.gov

Williams, Susan Nicole

OAK RIDGE NATIONAL LABORATORY

2706 Parside

McKinney, TX 75070 USA

972-658-1038

williamssn@ornl.gov

Wright, Eric L.

FACULTY AND STUDENT TEAM

PROGRAM (FaST)

13411 Blackstock Lane

Houston, TX 77083 USA

832-640-5464

ew3000_16@hotmail.com 
Wright, Richard Quinten

OAK RIDGE NATIONAL LABORATORY

104 W. Newcomb Road

Oak Ridge, TN 37831 USA

865-576-8251

3wh@ornl.gov

Young, Glenn Reid

OAK RIDGE NATIONAL LABORATORY

PO Box 2008, Bldg. 6025, MS-6369

Oak Ridge, TN 37831-6369 USA

865-576-2770

younggr@ornl.gov 


\section{ORELA Workshop Organizing Committee}

Michael Dunn, Program Chair

Nuclear Science and Technology Division, ORNL

Klaus Guber

Nuclear Science and Technology Division, ORNL

Paul Koehler

Physics Division, ORNL

Mike Westfall

Nuclear Science and Technology Division, ORNL

Glenn Young

Physics Division Director, ORNL 
xviii 


\title{
Nuclear Data Needs Supporting Gen-IV Applications- INL Perspective and Initiatives
}

\author{
D. W. Nigg, J. D. Baker, C. A. McGrath, C. L. Riddle, A. Caffrey, \\ J. Anderson, J. D. Cole, M. W. Drigert, R. Aryaeinejad, \\ Idaho National Laboratory, P.O. Box 1625, Idaho Falls, ID, 83415 USA \\ R. V. F. Janssens, B. J. Micklich \\ Argonne National Laboratory, Chicago, IL, USA \\ G. Ter-akopian \\ Joint Institute for Nuclear Research, Dubna, Russia
}

\section{INTRODUCTION}

Studies conducted in connection with the U.S. Department of Energy (DOE) Generation IV (Gen-IV) and Advanced Fuel Cycle (AFC) programs show that the transuranic nuclides can strongly influence the neutronic behavior of some advanced nuclear energy systems of interest. Essentially all integral nuclear parameters computed using modern reactor physics codes and data libraries are affected by propagation of uncertainty in the underlying nuclear data used in the computational models. These parameters include

- criticality (multiplication factor)

- reactivity feedback coefficients (e.g., Doppler, coolant void)

- $\quad$ kinetics parameters (e.g., effective delayed neutron fraction)

- reactivity loss during irradiation (burnup swing)

- peak power value

- conversion ratio of sustainable cores

- transmutation potential of burner cores

- max dpa, maximum helium and hydrogen production, etc.

- decay heat, radiotoxicity, and neutron and gamma radiation levels

However, the necessary cross-section information may be unavailable with the required accuracy from the current nuclear databases for some key nuclides of interest. As a specific example, recent sensitivity analyses for the Very High Temperature Gen-IV reactor concept [1-3], which features a somewhat harder thermal neutron spectrum and a significantly higher fuel burnup target than is the case for standard lightwater reactors, show a potential need for improved cross sections for some isotopes, including ${ }^{238} \mathrm{U},{ }^{239} \mathrm{Pu}$, ${ }^{240} \mathrm{Pu},{ }^{241} \mathrm{Pu}$, and ${ }^{243} \mathrm{Am}$, primarily in the resonance energy range, in order to satisfy the defined accuracy requirements on key computed integral parameters. Other transuranic nuclides may be of similar importance for some of the other Gen-IV concepts, although the primary needs appear to be currently focused on a few plutonium and americium isotopes. The target accuracies identified in these studies are very stringent and will be a challenge to achieve in many cases.

In this article we briefly review the conclusions and recommendations of recent international workshops on nuclear data needs for Gen-IV. Following that is a discussion of two specific activities undertaken by the Idaho National Laboratory (INL) to participate in the international effort to address these needs. 


\section{NUCLEAR DATA WORKSHOPS}

There have been three recent workshops on nuclear data needs for Gen-IV. The first was held at Brookhaven National Laboratory. The participants were primarily from U.S. national laboratories and universities, with a few European participants. The second workshop was organized as an embedded meeting at the American Nuclear Society Topical Meeting on Reactor Physics held in Chicago during April 2004 (PHYSOR04). The third workshop was held in Antwerp, Belgium, in April 2005 and was primarily organized by the European Institute for Reference Materials and Measurements in Geel, Belgium, and was sponsored by DOE, Atomic Energy of Canada, and Euratom. This latter workshop was a follow-on to the two previous workshops with broader international participation, including representatives from France, Germany, Belgium, Romania, Italy, the Netherlands, Russia, USA, Canada, South Korea, Finland, the Czech Republic, Japan, Mexico, and various international organizations, including IRRM (Geel, Belgium), OECD-NEA, and IAEA. There were discussions of fuel development, intercomparison of evaluations, sensitivity studies, and basic discussions of nuclear data measurementsproviding a broad perspective and context.

Key conclusions of the Brookhaven workshop included the following:

- High burnup operation of the VHTR might require reevaluation of transuranic data (cross sections, decay data, and fission yields). Differential measurements may be needed for selected nuclides.

- Fast spectrum systems (GFR, LFR, and SFR) to be used within a closed fuel cycle require additional evaluation of data for transuranic nuclides, particularly minor actinides, as well as integral measurements for validation of differential (basic) data and their processing tools.

- Nonconventional structural, coolant, or fuel-matrix materials may necessitate new evaluations or measurements of basic data.

- A systematic approach based on sensitivity and uncertainty analysis is required for further specifying data needs. (This led to the sensitivity studies published in 2005.)

- Currently available experimental facilities, equipment, accelerator targets, and personnel required to support necessary differential nuclear data measurements should be able to address the anticipated need for data. Key U.S. facilities identified include those at LANL, ORNL, RPI, and the INL experimental apparatus located at ANL/IPNS.

- There should be a strong emphasis on maintenance of the relevant experimental capabilities and on development of a single national collaborative effort, coordinated with relevant international activities, that will provide the necessary information, with appropriate levels of validation, in a manner that makes best use of what will almost certainly be limited financial resources.

- A coordinated mechanism should be developed to facilitate the acquisition, maintenance, storage, distribution, and community use of sample targets, especially purified stable isotopes and actinides. An assessment of the nuclear materials available for this effort should be performed.

Key conclusions of the Antwerp workshop were as follows:

- Data uncertainties and assessment of their impact are keys to improvement of reactor and fuel cycle codes. New approaches to measurement and evaluation will be important in reducing the current uncertainties.

- In current fuel cycle scenarios uncertainties in ${ }^{238} \mathrm{U},{ }^{239} \mathrm{Pu}$, some of the higher plutonium isotopes and possibly americium will be more important than the higher actinides (ANL and CEA Studies).

- Some attention to more accuracy in fission product yields appears to be needed. 
- Several nonfuel materials (Bi, Pb, C, Si, Zr) may need additional improvement.

- There is a clear need for improved covariance matrices to use in uncertainty studies.

\section{THE INL NUCLEAR DATA INITIATIVE}

The Idaho National Laboratory (INL) has made significant contributions to the international nuclear database over the years. Cross-section measurements historically were performed using various techniques at the Materials Test Reactor in the early days of the laboratory. More recently, the INL has focused its efforts in nuclear physics on fundamental studies of the fission process using spontaneous fission sources as well as gated accelerator neutron sources with fissionable targets. The current nuclear data initiatives undertaken by the INL involve collaborations with Los Alamos National Laboratory (LANL) and, separately, with Argonne National Laboratory. The effort builds on historical INL capabilities not only in nuclear physics but also in radiochemistry.

The collaboration with Los Alamos involves production of actinide targets for use in capture and fission measurements performed by LANL at the Los Alamos Neutron Science Center (LANSCE). The current focus is on capture measurements at the LANSCE Lujan Center using the DANCE (Detector for Advanced Neutron Capture Experiments) detector array. DANCE, shown in Figure 1, is a $4 \pi$ calorimeter with $\mathrm{BaF}$ scintillation detectors. Targets are currently prepared for use in DANCE using an electrodeposition process, although development of a direct metal vapor deposition process for this purpose is currently under development at INL. Figure 2 shows a ${ }^{239} \mathrm{Pu}$ target delivered to LANL by INL in June 2005. Future plans call for preparation of ${ }^{242} \mathrm{Pu}$ targets as well as additional targets for other actinide studies using the LANL apparatus.

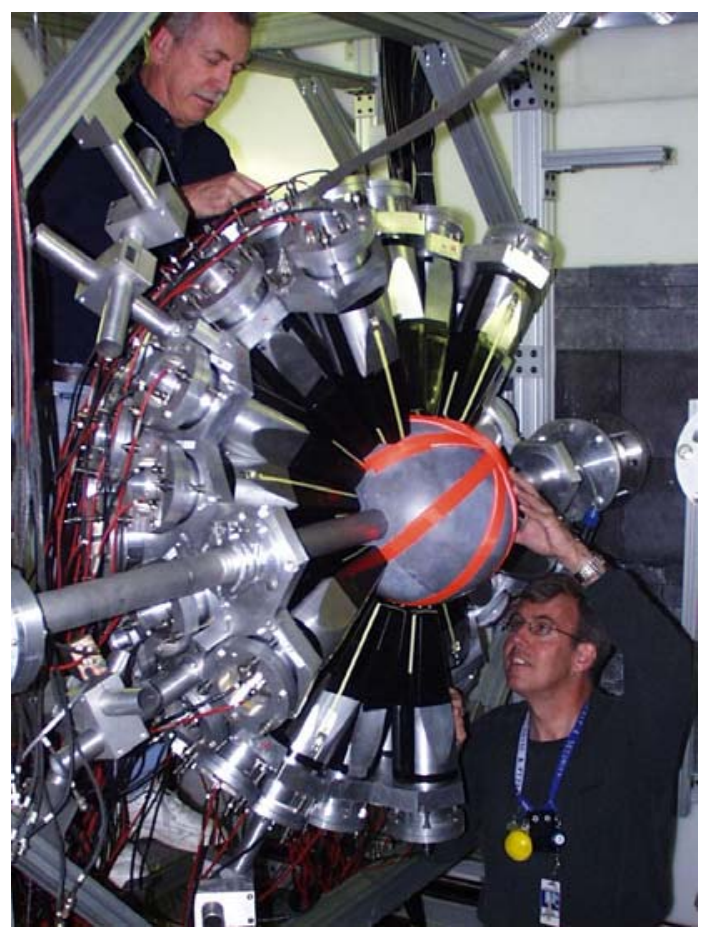

Figure 1. DANCE array under construction at LANL. Photo provided by J. Ullmann, LANL. 


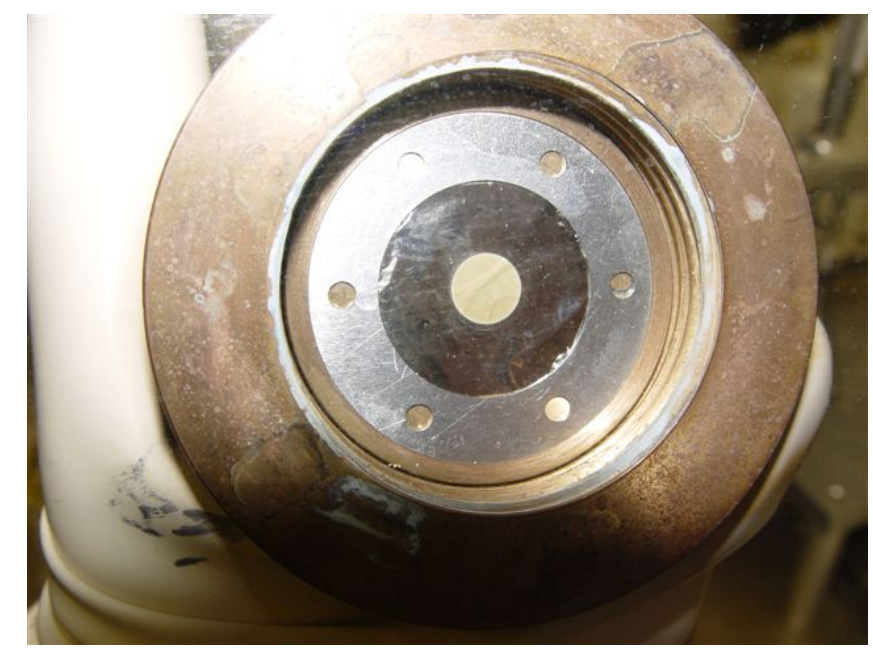

Figure 2. Plutonium-239 target prepared by INL for use in the LANL DANCE array.

The INL collaboration with ANL involves use of sophisticated detector arrays, supporting electronics, and data acquisition systems originally established by INL on a beamline of the Argonne Intense Pulsed Neutron Source (IPNS) Facility for use in fundamental studies of the fission process. The INL apparatus, which consists of an array of multiple types of multiple detectors operated in coincidence, with correlation of each observed event to the time of flight of the neutron that induced it, also offers an innovative method for cross-section measurements as well. The immediate measurement goals involve measurement of neutron-induced interaction cross sections for ${ }^{240} \mathrm{Pu},{ }^{242} \mathrm{Pu},{ }^{41} \mathrm{Pu}$, and possibly ${ }^{241} \mathrm{Am}$, with measurements for other nuclides of interest for advanced reactor physics applications to follow later.

The ANL/IPNS facility is a spallation neutron source with a moderated neutron beam that has a neutron spectrum at 12 and 20 meters, as illustrated in Figure 3. The results shown in Figure 3 are the average measured intensities and MCNP calculations performed at IPNS. For perspective, a direct comparison of flux intensity is shown between IPNS and the Los Alamos Lujan Center at lower neutron energies where explicit numbers, in similar units, are available, in particular for epithermal neutrons.

The uncertainty of the energy of a neutron that induces an interaction of interest in the target is determined largely by the uncertainty on the n-TOF from the time width of the proton pulse. In addition to this important parameter, the pulse rate and the flight path length interact to limit the useable energy range of the neutrons. At IPNS the pulse rate is $30 \mathrm{~Hz}$ and the proton pulse full width is $70 \mathrm{~ns}$. All beam lines at IPNS are heavily shielded and evacuated so that backgrounds are reduced. The low background at IPNS is an important factor for the long runs needed for experiments that achieve low statistical uncertainty. 


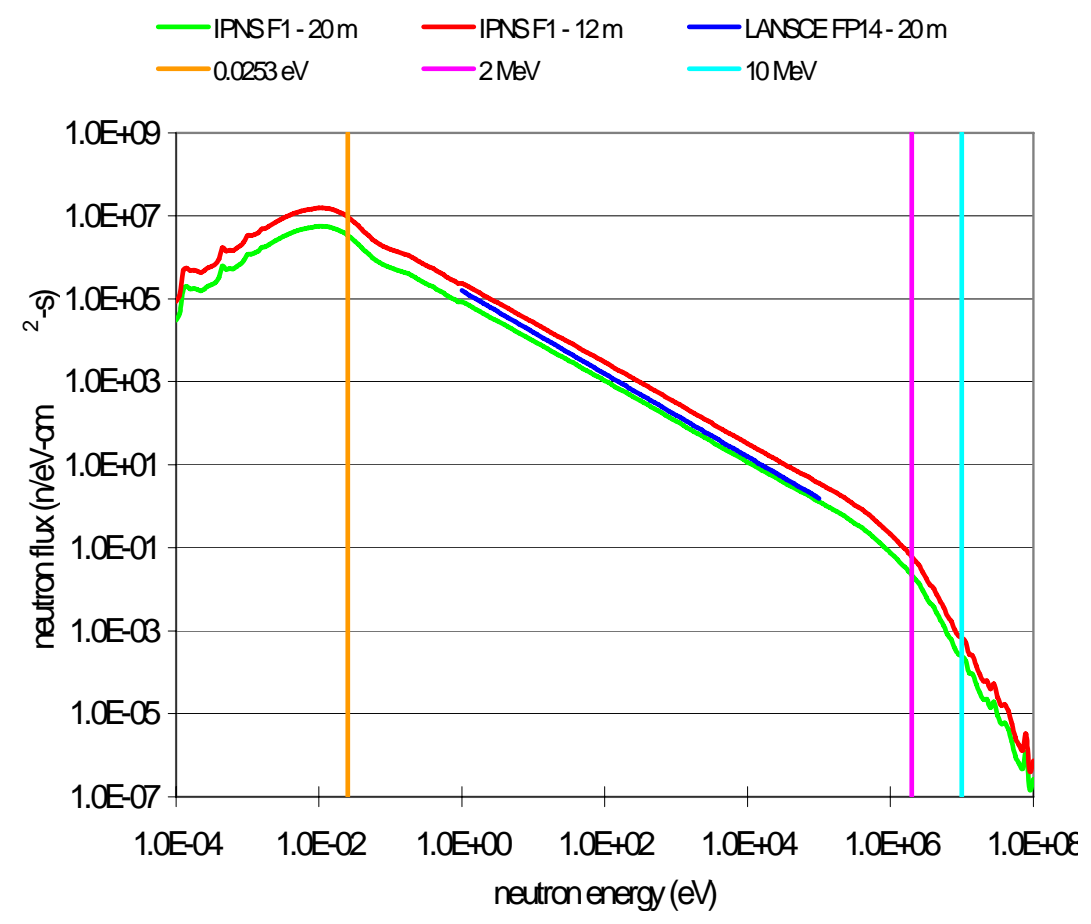

Figure 3. Plot of the neutron spectrum on the F3 beam line used for the INL apparatus for distances of 12 and 20 meters and the extrapolated values for FP14 at LANSCE. Energy markers are shown for the readers benefit.

The INL apparatus was originally installed at IPNS to perform experiments using induced fission of actinide targets for prompt information concerning fission yields by isotope pairs, nuclear structure information for prompt de-excitation of the fission products, multiplicity of both neutron and gamma rays by isotope pairs, and isotope pair distributions for fission cluster models. These efforts are extensions of spontaneous fission studies on ${ }^{252} \mathrm{Cf}$ and ${ }^{242} \mathrm{Pu}$ conducted with arrays of high-purity germanium (HPGe) detectors at INL, Oak Ridge National Laboratory (ORNL), Lawrence Berkeley National Laboratory (LBNL), and finally on GAMMASPHERE at both ANL and LBNL. This work has produced over 100 publications on the nuclear structure of fission products prior to beta decay, fission yields by isotope pairs, and explicit neutron multiplicity as correlated to specific fission pairs.

Two years ago efforts began to modify the INL apparatus at IPNS to provide the capability to measure neutron interaction cross sections as a function of incident neutron energy, branching ratios for the production of different isotopes by neutron capture or fission, and cross sections for the production of independent yields from actinide fission. The INL experimental technique allows a model-independent measurement of the neutron interaction cross section to be made over a continuous energy range from a few meV to above $2 \mathrm{MeV}$ without breaking the measurement into different energy segments. With approximately 4000 hours of beam time available for measurements in one year, low statistical uncertainty can be achieved.

The INL detector array, shown in Figure 4, is composed of 12 Compton-suppressed, high-purity germanium (CSHPGe) detectors, eight fast neutron detectors (BC501 liquid scintillator), and a stack of up to 32 silicon ( $\mathrm{Si}$ ) detectors interleaved with double-sided foils of actinide targets. The trigger electronics 
starts the digitization process if two of the CSHPGes, two neutron detectors, or a CSHPGe and a neutron detector produce a signal within a set coincidence time window. In this way three separate conditions can be used as independent triggers for determining whether a neutron interaction has occurred in a target. The coincidence is based on overlap timing with a time window of 50 to 100 nanoseconds.

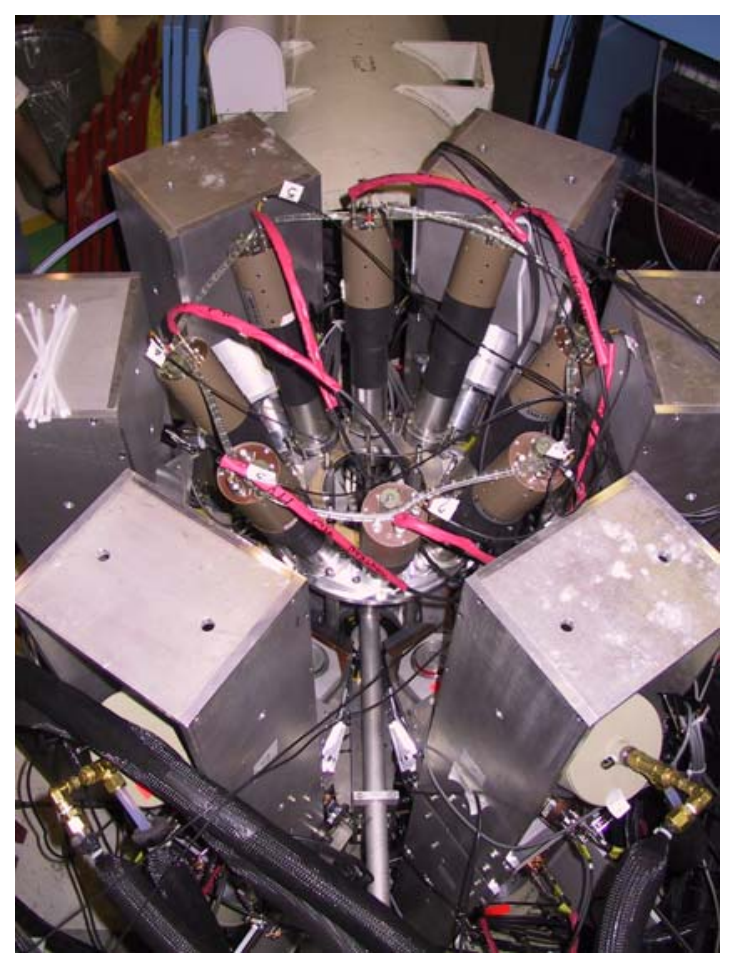

Figure 4. INL detector array for nuclear data measurements at ANL/IPNS.

The Si detectors are used to directly detect the fission fragments as these fragments recoil directly into the Si detectors. The Si detectors and the target foils are interleaved. Each actinide foil has a selected thickness that will allow the low-energy, light-mass fission fragment to escape the target and enter the Si detector, which is in contact with the target material. A discriminator is used to reject $\alpha$ particles and their pileup signal and accept only the fission fragment signal, which is a factor of ten greater in amplitude. These fission fragment signals in the Si detectors are used as a fourth trigger in the system. Si detectors are used instead of a fission chamber, primarily because the rise time of the output pulse is faster by roughly a factor of ten. In addition, since the Si detector is in contact with the actinide target, gamma rays observed from the fragment have no Doppler shift or broadening due to emission in flight. There are other advantages with size, less support electronics, better $\alpha$-particle discrimination, and low mass material. The energy output of each Si detector is also digitized and included in the data packet.

Signals from the four triggers are combined logically in various ways to produce an event signal. This event signal is used to trigger data acquisition of digitized detector signals, time relationships between the detector signals, and as a stop on a multi-stop time-digital converter (TDC). In this way a multiparameter data packet is acquired for each observed radiation event and is stored in list mode format. Since the system is configured to respond to coincidence events and the prompt timing of an event is from $10^{-22}$ seconds to $10^{-12}$ seconds, a single trigger can result in multiple radiation types being included in the event. The simplest example is that of a fission fragment being detected in an Si detector and single gamma ray or neutron radiation also observed as will be shown later. 
With the ability to select the events by post-experiment sorting the multiplicities of both neutrons and gamma rays can be determined. The production rates of the selected gamma rays arising from the prompt fission fragments or the excited isotopes produced by neutron capture are used to determine the appropriate reaction cross sections as a function of the incident neutron energy, determined from the event-by-event neutron TOF parameter. In addition to information required to extract absolute reaction cross sections, the acquired data sets also will contain the information needed to extract independent fission fragment yields that can be used to validate accepted values in a model-independent manner.

The most important aspect of this powerful ability to select events by sorting and then determining cross sections is to reduce background and events unassociated with the reaction channel of interest. This reduces the error on the cross section by allowing an event set to be selected that only contains events from that particular reaction channel. For example, problems associated with measuring the fission cross section for material with high spontaneous fission rates can be handled by sorting events based on different conditions. This method also reduces the total error whereas the traditional method of beam-on, beam-off does not remove the spontaneous fission events from the beam or data set. Selecting fission events by requiring an Si detector signal and then sorting gamma rays from different fission pairs will provide information on contributions for the two types of fission processes. The distribution of neutrons and thus associated fragment pairs from spontaneous fission are different for those produced by induced fission. This is caused by the differences in the excitation energy of the fissioning nucleus. In spontaneous fission the nucleus is in its ground state. For induced fission the nucleus will be at an excited state due to the energy brought in by the incident neutron and by the rearrangement of the population of the nuclear orbitals in the nuclear system after the neutron is captured.

The INL apparatus at IPNS thus has some key unique capabilities supported by the IPNS facility itself. The most important is the ability to take coincidence data associated with a particular nuclear event. An array of detectors could be operated in this manner at other facilities as well, but at IPNS two important enabling features are available: (1) an intense flux of neutrons and (2) the availability of the beam for long experimental measurements. These two facts allow low statistical uncertainties to be attained. Achieving a goal of $\sim 10^{9}$ events observed and stored by the data system requires over 100 days of beam time. This long experimental time is available at IPNS since the INL apparatus is on one beam line and does not affect experiments at other locations in the facility. The other unique capability associated with the INL protocol is related to the ability to perform nuclear-event-based data collection and post-event analysis by sorting data into subsets based on physics conditions. By imposing multiple conditions and sorts via software or computer processing in selecting data sets for detailed analysis, the "cross talk" between channels can be minimized in ways that are not possible in the hardware of the electronics. The simplest and easiest to understand is the Si detector trigger to separate fission events and all other events. The nonfission events can be further sorted. This capability has produced exceptional results in nuclear structure and spontaneous fission studies and is also applicable to the problems of measuring various neutron cross sections of actinide isotopes.

The post-event analysis capability based on data selection of reaction channels provides results that are self-consistent across the larger experimental data set. This means that the ability to use different approaches in sorting can provide results that provide consistency checks that are otherwise not available. An example of this is the case of the determination of a fission cross section by direct selection of the observation from the Si detectors and the cross section determined from sorting on gamma rays from the highest-yield fission fragments. Although the statistics in these two subsets of data will be different, the cross section should have the same result in both cases. This is a powerful tool to check the results and to provide a consistency not found in previous work. 
Targets used by INL at the IPN facility are fabricated in Russia by collaborators at the Joint Institute for Nuclear Research (JINR). The targets are metal foils, not oxides, on an appropriate backing and of the thickness needed to allow the light fission fragments to escape the target and enter the silicon detector that is in contact with the target material. This capability removes the need for large corrections of the incident neutron flux that must often be done in the case of oxide targets. In addition, the vapor deposition of metal onto a metal backing gives excellent stability to the targets and reduces the risk of contamination due to targets coming off the backing. The isotopic purity of the targets is greater than 98\% for the principal isotope. A detailed chemical analysis is provide for each target batch, and individual target characteristics, such as mass per unit area and total mass are provided.

Authorization was received in January 2005 for internal INL funding to perform a proof of principle experiment for cross section measurements at IPNS using ${ }^{239} \mathrm{Pu}$ standards. This experiment was recommended in a September 2004 international peer review of the INL/ANL proposal to perform some types of nuclear data measurements pertinent to the VHTR/Gen-4 program at IPNS. The experiment was successfully initiated in early May 2005, and data collection for the most recent accelerator operation cycle continued until the end of the cycle in late June. Significant results were obtained and were presented at the DOE AFCI/Gen-IV Physics Working Group meeting at ANL (July 19-20). Some key initial results are summarized below.

Figure 5 shows some initial time-of-flight (TOF) correlated fission event spectrum data from the INL silicon detectors over the energy range of interest from thermal to about $1 \mathrm{MeV}$. The prominent first resonance in plutonium at $0.3 \mathrm{eV}$ is labeled, as are a few of the higher resonances. (As one goes to the left in this plot the incident neutron energy increases to first order as the inverse square of the time of flight.)

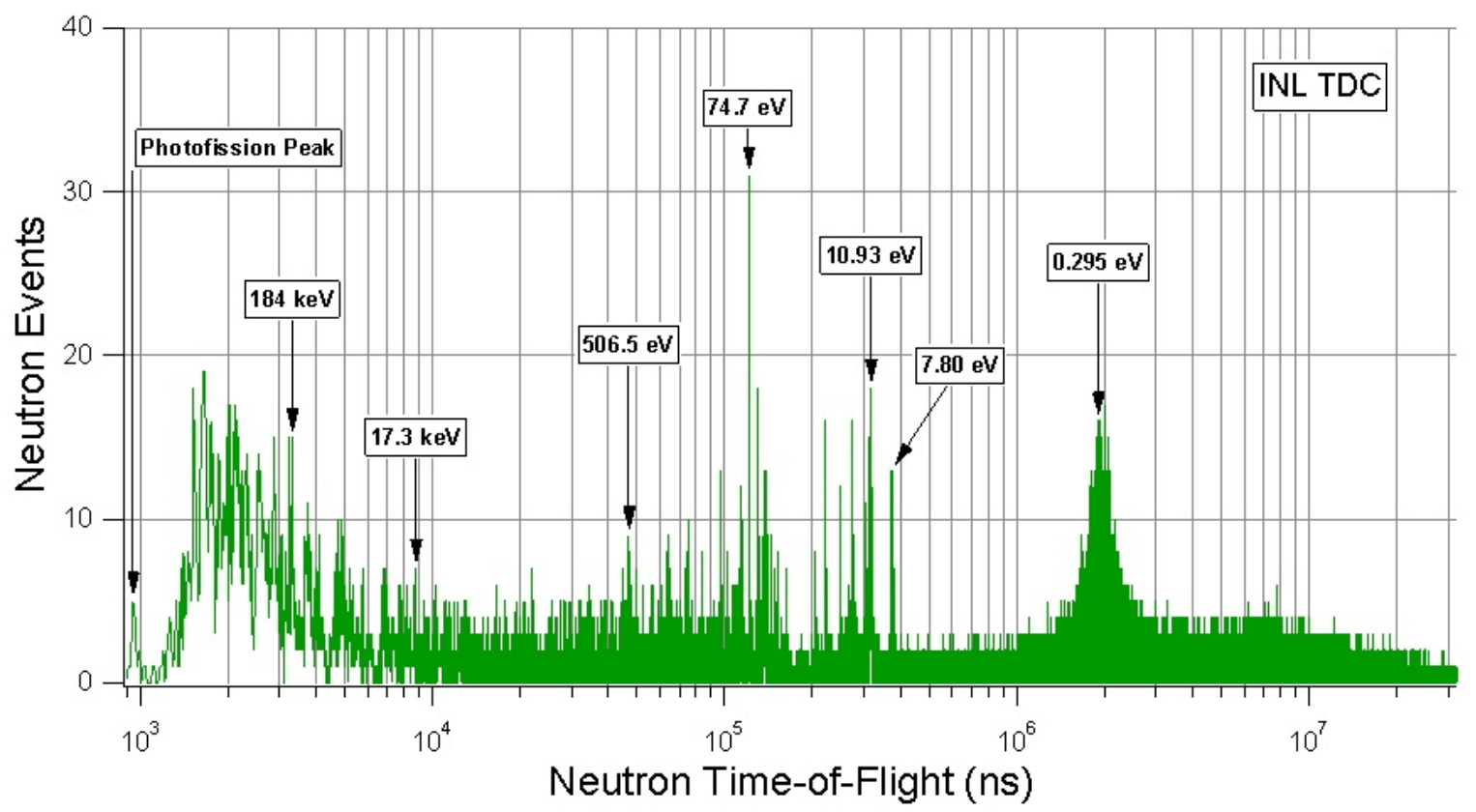

Figure 5. Initial ${ }^{239} \mathrm{Pu}$ Fission event spectrum from the IPNS nuclear data proof of principal experiment at ANL/IPNS. The neutron energy range spans eight decades. 
Figure 6 shows the same data with the energy range up to about $500 \mathrm{eV}$ expanded to show more detail of the measured data for the prominent resonances.

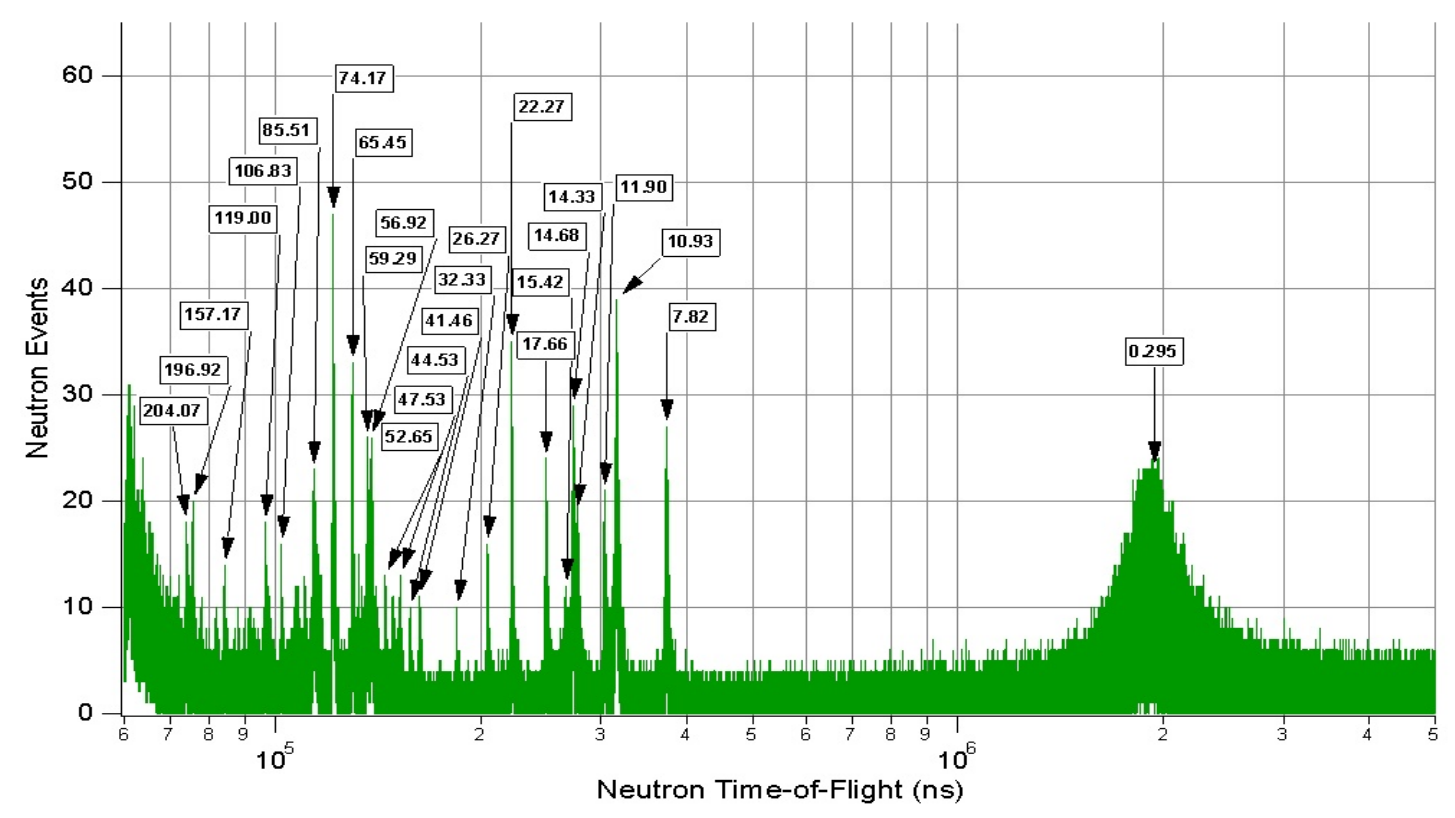

Figure 6. Fission event spectrum for ${ }^{239} \mathrm{Pu}$. Expanded energy scale.

Figure 7 shows a further expansion of the energy scale to show detail in the energy range around $200 \mathrm{eV}$. The measured data are given in the lower plot, while the ENDF evaluation of the corresponding cross section for the same energy range is given in the top plot for comparison. Note the remarkable fidelity of the measured data relative to the standard evaluation. The statistical uncertainty on the measurements in these preliminary results is on the order of $10 \%$ at the resolution shown. However, these early demonstration experiments only involve collection of data over a few days of run time. In a precision calibration, or for a measurement of the cross section for an unknown sample, the run time would be much longer to permit greater statistical accuracy, on the order of the requirements identified by the various uncertainty propagation studies. 

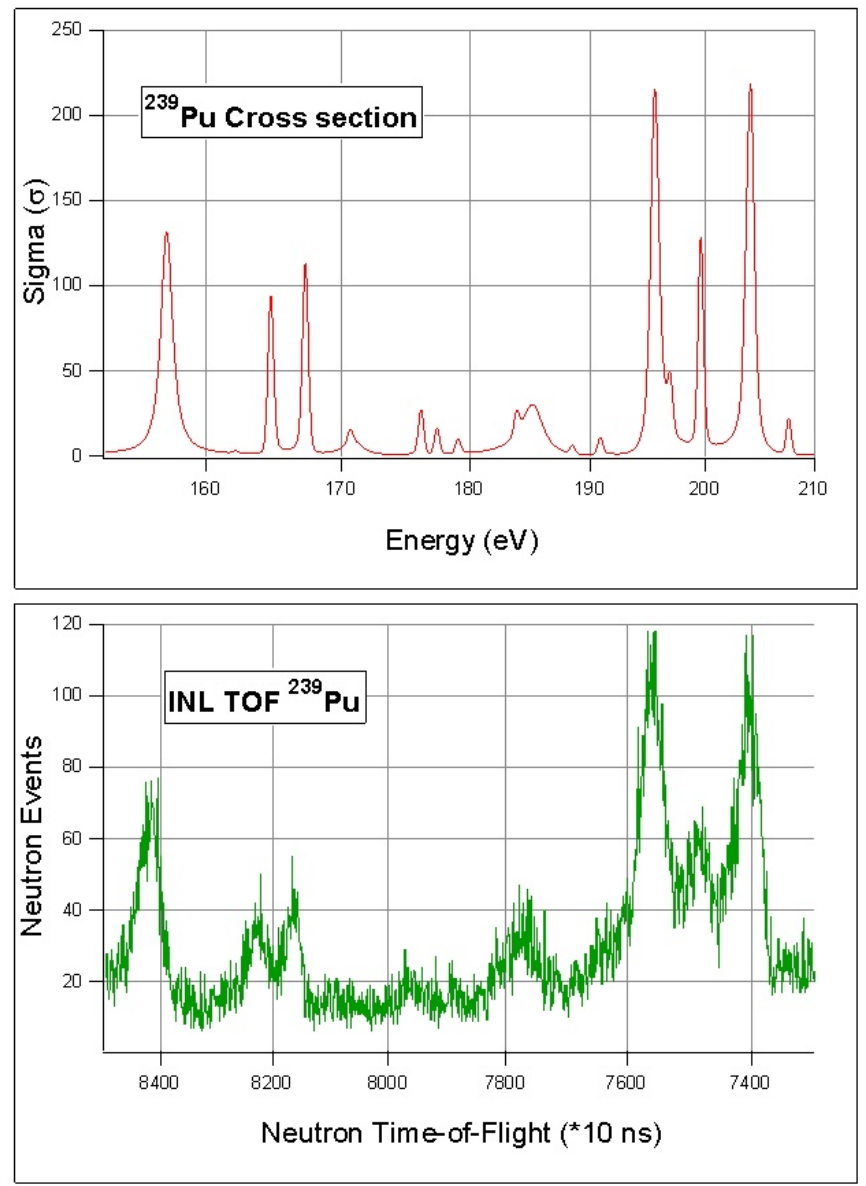

Figure 7. Comparison of the ENDF fission cross section (top) with the INL measured fission event spectrum.

Finally, and most significantly, it should be noted that the results given in Figures 5-7 are for "singles" data, i.e., data from the silicon fission detectors alone, with no coincidence gating. Figure 8 provides a striking initial example of what can be achieved by coincidence gating. In this case the signals from the gamma detectors in the INL/IPNS detector array are gated (by post-experiment sorting) in anticoincidence with the signals from the silicon fission detectors. This offers a self-consistent way of separating neutron capture events from fission events in a way that avoids the need to do individual experiments for each, or to normalize data to computational models. That is, an event detected by the gamma detectors is only "counted" if a fission is not detected at the same time. Figure 8 shows the results of this procedure for the energy range around $10 \mathrm{eV}$. The top plot once again shows the ENDF cross section (the standard for comparison) and the bottom plot shows the gated data, i.e., the neutron capture event spectrum (proportional to the capture cross section), separated from the fission event spectrum solely by experiment, in the same experiment—a key feature of the coincidence approach used at IPNS. 

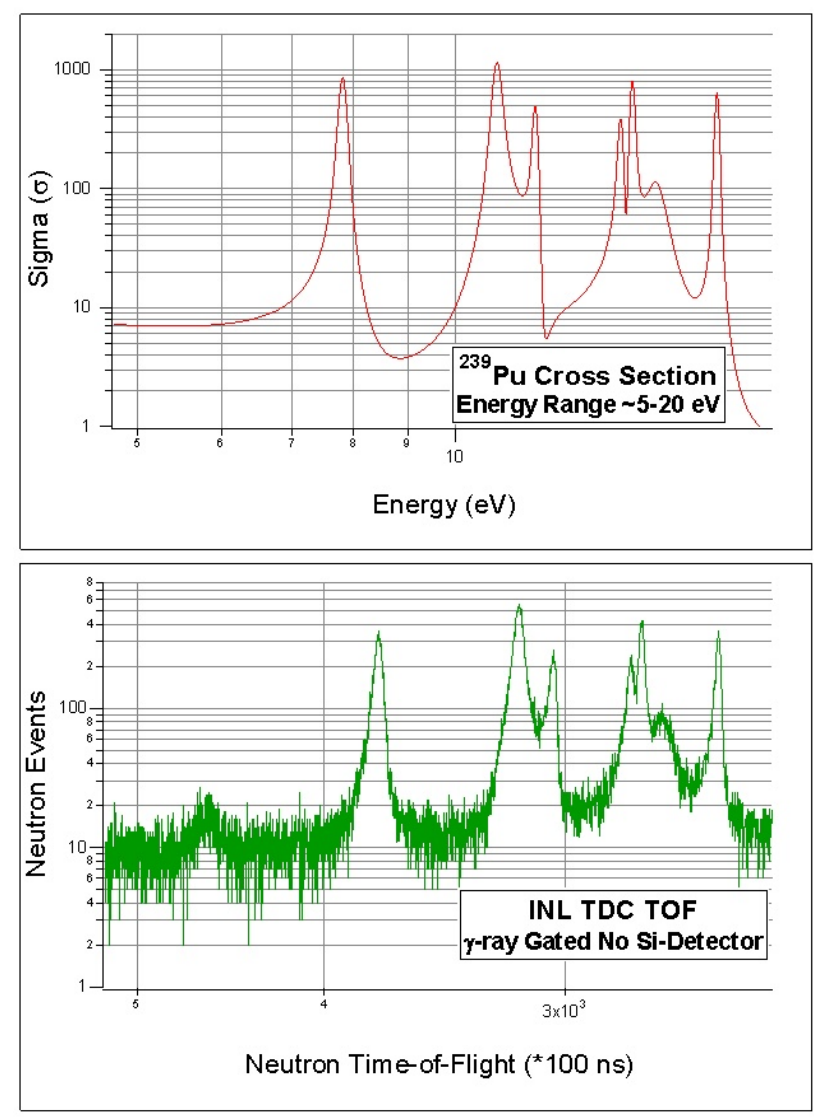

Figure 8. Capture event spectrum (bottom) for ${ }^{239} \mathrm{Pu}$ measured by INL using anticoincidence with fission, compared with the ENDF capture cross section evaluation (top).

Once again the high fidelity of the measured data is apparent. Furthermore, the coincidence approach also allows many other types of signals to be separated. For example, separating a neutron-induced event spectrum (i.e., cross section) from the very high background radioactivity present in some actinide targets of interest is very effectively enabled by this technique. Compensation for unwanted background due to target contamination is also possible. It may also be possible to resolve resonances in a manner that is not limited by the proton pulse width of the accelerator by capturing, through coincidence techniques, differences that may exist between the decay schemes of each resonance, although this remains to be demonstrated. Taken together, these features of the experimental apparatus and protocol offer the potential for improved accuracy in differential actinide cross section measurements important to VHTR as well as to other Gen-IV concepts.

\section{REFERENCES}

1. T. A. Taiwo and H. S. Khalil (2005a) "Nuclear Data Needs for Generation IV Nuclear Energy Systems - Summary of U.S. Workshop," International Workshop on Nuclear Data Needs for Generation IV Nuclear Energy Systems, Antwerp, Belgium, April 5-7, 2005.

2. G. Aliberti, G. Palmiotti, M. Salvatores, T. K. Kim, T. A. Taiwo, I. Kodeli, E. Sartori, J. C. Bosq, and J. Tommasi (2005) Sensitivity of Advanced Reactor and Fuel Cycle Performance Parameters to 
Nuclear Data Uncertainties," International Workshop on Nuclear Data Needs for Generation IV Nuclear Energy Systems, Antwerp, Belgium, April 5-7, 2005.

3. T. A. Taiwo, G. Palmiotti, G. Aliberti, M. Salvatores, and T. K. Kim (2005b) "Uncertainty and Target Accuracy Studies for the Very High Temperature Reactor (VHTR) Physics Parameters,” ANL-GenIV-051, August, 2005. 


\title{
NNDC Perspective on Nuclear Reaction Databases and ORELA
}

\author{
Pavel Oblozinsky \\ National Nuclear Data Center, Brookhaven National Laboratory \\ P.O. Box 5000, Upton, NY 11973
}

\begin{abstract}
We discuss perspective of the National Nuclear Data Center on nuclear reaction databases, focusing on contributions from the ORELA machine over more than 3 decades. We conclude that ORELA had profound impact on nuclear reaction databases, in particular CSISRS and ENDF. In addition, ORELA contributed considerable amount of data included in the recent Atlas of Neutron Resonances, along with data of critical importance for nuclear reaction model calculations by codes such as EMPIRE.
\end{abstract}

\section{INTRODUCTION}

The ORELA machine, commissioned in 1971, is unique experimental facility that produced a remarkable amount of neutron cross-section data and played a crucial role in the development of U.S. nuclear reaction databases over more than three decades.

We start our discussion by displaying the front page of the web service of the National Nuclear Data Center (NNDC) in Figure 1. Eight databases that can be seen at the top left portion of the figure include both nuclear reaction and nuclear structure databases [1]. Nuclear reaction databases (CINDA, CSISRS, ENDF) are shown in green; nuclear structure databases (XUNDL, ENSDF, MIRD) in blue, while mixed databases (NSR, NuDat) are shown in green-blue colors.

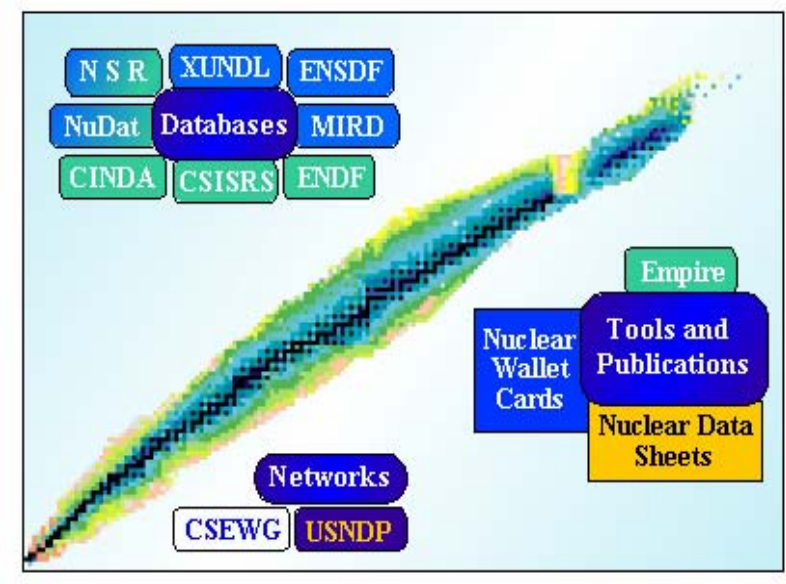

Figure 1. Front page of the web service of the National Nuclear Data Center (NNDC), www.nndc.bnl.gov, shows the NNDC portfolio of nuclear databases at top left.

In addition to basic nuclear reaction databases, we want to highlight two other products with important impact from ORELA, namely, Atlas of Neutron Resonances which should be published in 2006 [2], and nuclear reaction model code EMPIRE [3], which serves as advanced tool for nuclear reaction evaluations in fast neutron region. 


\section{BASIC NUCLEAR REACTION DATABASES}

The ORELA machine provided considerable input to several basic nuclear reaction databases:

- the bibliography databases CINDA and NSR,

- the experimental reaction database CSISRS, and

- the evaluated reaction database ENDF.

\section{Bibliography Databases}

CINDA (Computer Index to Nuclear Data) is the oldest nuclear database, containing references to 275,000 neutron-induced reactions from 55,000 works. The database was traditionally dedicated to neutron data and in this area it is widely considered to be comprehensive. In 2005, the database was extended to cover charged-particle reactions (in general, light incident charged-particles with mass A $<12$ and energy $\mathrm{E}<1 \mathrm{GeV}$ ) and photonuclear reactions. In the last couple of years, CINDA is losing its role as the primary database and it is taking over a role of an index to experimental database CSISRS.

NSR (Nuclear Science References) database contains references describing contents of 180,000 articles from more than 80 journals. The database, initially known as Nuclear Structure References, evolved into a broad bibliography database for nuclear science. The strength of the database is in keywords that allow efficient search over research topics, nucleus, reaction, author etc. The database is updated weekly by the NNDC, and it contains also references on neutron-induced reactions.

Neither CINDA nor NSR provides information on facilities making it somewhat difficult to quantify ORELA impact. A more favorable situation in this respect is with the experimental reaction database CSISRS where this information can be extracted relatively easily.

\section{Experimental Database}

CSISRS (Cross Section Information Storage and Retrieval System) is one of the key nuclear reaction databases, containing numerical information on experimental cross-section data. The database is also known as EXFOR (Exchange Format), a name derived from the mechanism of worldwide exchange of information among nuclear reaction data centers. The database currently contains cross sections from more than 15,500 experiments. Neutron reactions are considered to be covered to almost $100 \%$, chargedparticle reactions (up to some excuses these are reactions induced by light charged-particles with A $<12$ and $\mathrm{E}<1 \mathrm{GeV}$ ) are covered much less, while photonuclear reactions are covered only to about $20 \%$.

Information about contributions coming from the ORELA facility can be readily obtained from the CSISRS database. Results are summarized in Figure 2. In total, CSISRS contains experimental data from 334 papers produced by ORELA since 1971, with the peak productivity in the period of 1976-1980. In the last 10-15 years, ORELA productivity is down to the level of a few papers per year. 


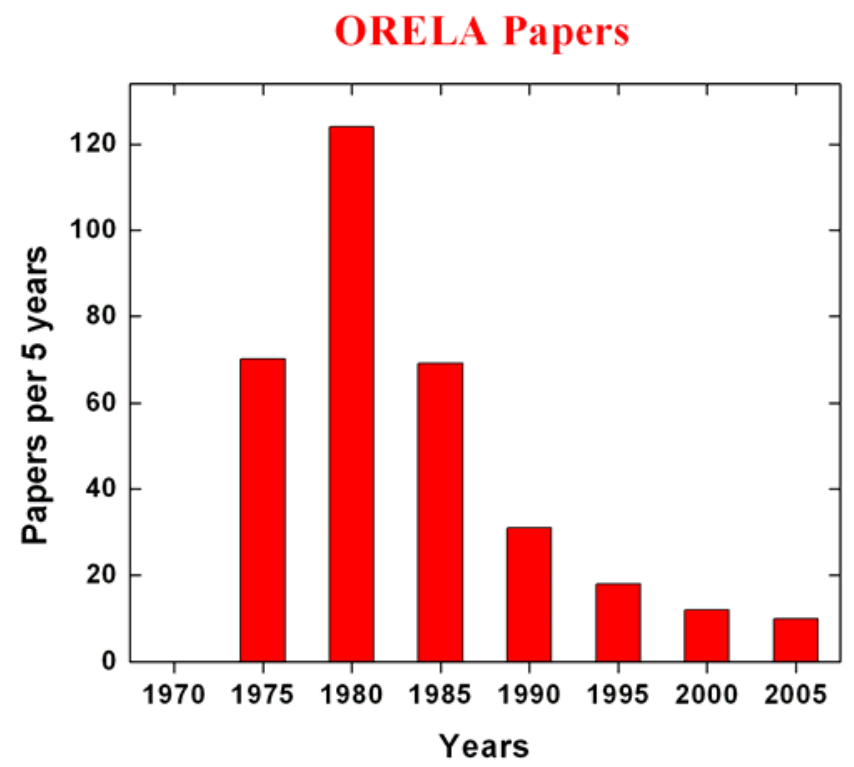

Figure 2. Number of ORELA papers compiled into experimental database CSISRS.

\section{Evaluated Database}

ENDF (Evaluated Nuclear Data File) is the database that contains evaluated nuclear reaction cross-section data for all nuclei relevant to applied nuclear technologies. The database currently covers 328 target materials (315 isotopes and 13 elements), interacting mostly with neutrons. However, interactions with protons and other light charged particles are also included. Incident energies up to $20 \mathrm{MeV}$ are mainly covered, but important cases with energies up to $150 \mathrm{MeV}$ are also included.

The current version of the database, ENDF/B-VI.8, was released in October 2001. A new version of the database, ENDF/B-VII, will be released in FY 2006. The database provides input to neutronics calculations, such as design of reactors, criticality assemblies, accelerators, as well as radiation shielding and protection.

ORNL contributions to ENDF, as summarized in Table 1, are largely based on ORELA measurements. In the current ENDF/B-VI.8, majority of evaluations supplied by ORNL falls in the category of fission products. Number of ORNL evaluations in the new ENDF/B-VII will most likely decrease somewhat, but this is more than offset by several important contributions in the actinide region. In addition, ORNL should supply 10 materials with covariance data in the resonance region, of importance to criticality safety applications. These data are produced by a new retroactive method that first simulates ORELA experiments, followed by their analysis to deduce covariances. 
Table 1. ORNL \& ORELA contribution of evaluated neutron reactions in the ENDF/B-VI.8 database released in 2001 and ENDF/B-VII database (2006)

\begin{tabular}{lcc}
\hline \multicolumn{1}{c}{ Materials } & ENDF/B-VI.8 & ENDF/B-VII \\
\hline $\begin{array}{l}\text { Total number of } \\
\text { materials }\end{array}$ & 328 & 348 \\
$\begin{array}{l}\text { Number of materials } \\
\text { from ORNL \& }\end{array}$ & $\begin{array}{l}\text { Mostly resonances for } \\
\text { fission products }\end{array}$ & $\begin{array}{l}\text { Several important actinides, } \\
10 \text { materials with covariances }\end{array}$ \\
\hline
\end{tabular}

\section{OTHER NUCLEAR REACTION DATABASES AND TOOLS}

In addition to basic nuclear reaction databases, ORELA contributed a considerable amount of data or supporting information for two other important nuclear reaction products maintained by the NNDC:

- Atlas of Neutron Resonances, and

- Nuclear Reaction Model Code EMPIRE.

\section{Atlas of Neutron Resonances}

Atlas of Neutron Resonances, authored by Said Mughabghab of the NNDC, is currently under completion. Elsevier should publish this impressive treatise in two volumes in 2006. The Atlas continues the tradition of well-known report BNL-325 and it represents its 5th edition. The previous 4th edition, published in two volumes by Academic Press in 1981 and 1984, was widely used and frequently cited.

The Atlas contains parameters of neutron resonances and thermal cross sections as well as average resonance parameters and quantities for 473 isotopes for all elements $Z=1-100$. The only exception is $\mathrm{Z}=85$ and 87 that are not covered because of entire lack of experimental data. The Atlas provides data resulting from careful evaluation by the leading expert with decades of experience and reputation in the neutron resonance field.

A large amount of data from ORELA measurements is used in the Atlas. In particular, one should mention numerous measurements produced in the past primarily by Harvey and Macklin, and more recent data measured primarily by Paul Koehler and Klaus Guber. These data were mostly dealing with structural materials, fission products and nuclei in the Lu-Pb region, with heavy nuclei such as ${ }^{232} \mathrm{Th}$, ${ }^{231} \mathrm{~Pa},{ }^{233,238} \mathrm{U},{ }^{237} \mathrm{~Np}$, and ${ }^{241,242 \mathrm{~m}} \mathrm{Am}$, and also with lighter nuclei ${ }^{16} \mathrm{O},{ }^{19} \mathrm{~F}$ and ${ }^{35,37} \mathrm{Cl}$.

\section{Nuclear Model Code EMPIRE}

The ORELA machine provided the experimental basis for other data of considerable interest. In particular, these data are important for model calculations, such as average resonance spacings $\mathrm{D}_{0}$ for nuclear level densities, neutron radiative widths for photon strength functions, and neutron strength functions for optical model parameterization.

As an example we show in Figure 3 nuclear level densities for the ${ }^{157} \mathrm{Gd}+\mathrm{n}$ system as used in the nuclear reaction model code EMPIRE [2]. Level densities are normalized, to discrete levels at low energies and 
to $1 / \mathrm{D}_{\mathbf{0}}$ at neutron binding energy, to get a realistic description of this quantity, probably the most important quantity for neutron cross-section calculations at low energies.

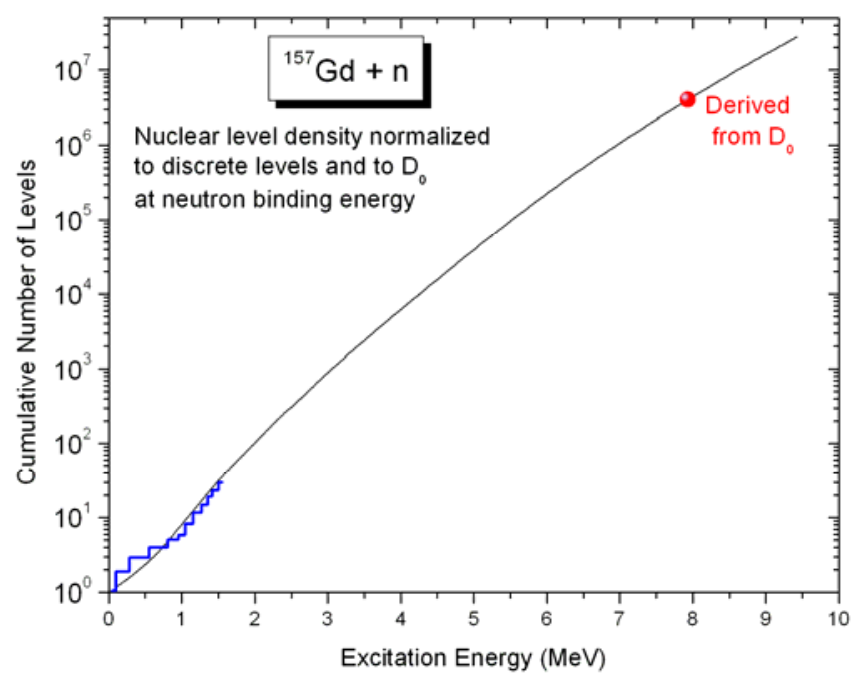

Figure 3. Nuclear level density for ${ }^{157} \mathrm{Gd}+\mathrm{n}$ normalized to discrete levels at low energies and to $1 / \mathrm{D}_{0}$ at neutron binding energy.

\section{CONCLUSIONS}

The ORELA machine, largely thanks to its exceptional capabilities in high-resolution neutron resonance cross section measurements, has made a profound impact on neutron reaction data and on nuclear reaction databases. By producing numerous experimental data of critical importance, the ORELA machine put its unique and lasting imprint on nuclear technology applications as well as on nuclear reaction physics.

\section{REFERENCES}

1. Nuclear Reaction Databases of the National Nuclear Data Center, www.nndc.bnl.gov.

2. S. Mughabghab, Atlas of Neutron Resonances (Elsevier, 2006), to be published.

3. M. Herman et al., Nuclear Reaction Model Code EMPIRE, www.nndc.bnl.gov/empire. 


\title{
Oak Ridge National Laboratory Neutron Sciences Overview
}

\author{
Paul E. Koehler \\ Physics Division, Oak Ridge National Laboratory, P.O. Box 2008, Oak Ridge, TN 37831
}

\begin{abstract}
The neutron sciences program at Oak Ridge National Laboratory (ORNL) is based at two major facilities, the Spallation Neutron Source (SNS) and the High Flux Isotope Reactor (HFIR), and a third, smaller facility, the Oak Ridge Electron Linear Accelerator (ORELA). By far the largest component of the program is in the field of neutron scattering, but there also are significant programs in isotope production, fundamental neutron physics, and basic and applied nuclear physics. I give a brief sketch of these three facilities and some of the research conducted at them, concentrating on the areas of most interest to this workshop.
\end{abstract}

\section{INTRODUCTION}

While surfing the web, if you catch the wave taking you to the external ORNL home page, you'll see a button labeled "Neutron Sciences." If you click this button, you'll learn that the field of neutron sciences at ORNL is dominated by two whale-sized facilities - the SNS and the HFIR. If you continue to surf to the nether regions, you'll find that there is a smaller, perhaps swordfish-sized, facility called ORELA, at which neutron science research also is done. Together, these three facilities present a wide range of capabilities and hence a broad spectrum of research in the neutron sciences. In the next section, I briefly will describe each facility. In the following section, I will give examples of the research, concentrating on areas of most interest to this workshop.

\section{ORNL’S THREE NEUTRON-SCIENCE FACILITIES}

HFIR [1] was first of the three facilities to come on line, with full-power operation starting in 1966. The HFIR reactor runs at $85 \mathrm{MW}$ using highly enriched ${ }^{235} \mathrm{U}$ fuel which is cooled and moderated by light water and reflected by Be in a flux-trap design. There are numerous irradiation positions, some of which allow insertion and removal of samples while the reactor is running, into fluxes as high as about $2 \times 10^{15}$ neutrons $/ \mathrm{cm}^{2} / \mathrm{s}$. There are four horizontal beam tubes as well as a new cold source and experiment hall. The facility is in the midst of an upgrade. Eventually, there will be 15 neutron scattering instruments, five of which will be part of the user program.

ORELA [2] came on line just a few years after HFIR, in 1969. Neutrons are generated at ORELA via photoneutron reactions when electrons from the accelerator slow down in a Ta target. Water cooling for the target also serves as a moderator. The resulting flux spans the energy from thermal to above $40 \mathrm{MeV}$. The electron beam from the ORELA accelerator is pulsed; hence, neutrons emanate from the ORELA target in pulses. This allows the energy of a neutron interacting with a sample or detector placed in the neutron beam to be determined using a time-of-flight technique. Because the width of the ORELA beam pulses are short (variable between 2 and $30 \mathrm{~ns}$ ) and distances between the neutron source and experiment can be relatively large (8-200 m), the energy of an interacting neutron can be determined with very good precision. This excellent energy resolution at ORELA can be very valuable for making high-accuracy cross-section measurements. Also, because the repetition rate at ORELA is variable between 1 and $1000 \mathrm{~Hz}$, the beam conditions can be tailored to suit a wide variety of experiments. 
Currently, there are nine beam lines at ORELA available for experiments and four standard "instruments" for neutron cross-section measurements. In addition, several one-of-a-kind experiments often are mounted on some of the flight paths.

The Spallation Neutron Source (SNS) is the newest neutron science facility at ORNL. The SNS is scheduled to begin operation in the last quarter of 2006. Neutrons will be generated at the SNS via spallation when a 1-GeV proton beam from the linear accelerator strikes a mercury target. The baseline design [3] is to achieve an average current of $1.4 \mathrm{~mA}$ for an average power of 1.4 MW. Pulses of protons of about 700 ns duration (with a FWHM of about $350 \mathrm{~ns}$ ) will be delivered to the spallation target at a repetition rate of $60 \mathrm{~Hz}$. The neutron flux at the SNS is expected to be up to ten times higher than any other such source in the world [4]. There will be four moderators surrounding the mercury target-three containing cold hydrogen and one containing ambient water. There will be 18 beam shutters and a total of 24 beam lines at the SNS [3].

\section{NEUTRON SCIENCE AT ORNL}

Neutron scattering is by far the largest component of the neutron sciences program at ORNL. All of the 15 instruments at HFIR are designed for neutron scattering experiments or to test components for neutron scattering beam lines. Of these 15, five are part of the user program and seven view the new cold source. All but one of the beam lines approved so far at the SNS will house instruments for neutron scattering. Five of the planned instruments are considered part of the SNS whereas the rest are being fielded by instrument development teams (IDTs). With these instruments, neutron scattering will be used to study problems in chemistry, complex fluids, crystalline and disordered materials, engineering, magnetism and superconductivity, polymers, and structural biology.

A substantial component of the program at HFIR is devoted to isotope production, activation analysis, and materials irradiation. HFIR is the West's sole source [5] of ${ }^{252} \mathrm{Cf}$, which is used in several medical, industrial, and scientific applications. Other isotopes produced at HFIR include ${ }^{43} \mathrm{~K}$, which is used for evaluation of coronary heart disease; ${ }^{103} \mathrm{Pd}$, which is used in the treatment of prostate cancer; ${ }^{153} \mathrm{Gd}$, which is used to measure bone loss; and ${ }^{188} \mathrm{~W}$, which is associated with treatment of cancer and arthritis.

The single beam line at HFIR and SNS not earmarked for neutron scattering will be devoted to experiments in fundamental neutron physics. The IDT building beam line 13 at the SNS, a collaboration of several national laboratories and universities, will be operated by the Physics Division at ORNL. Four choppers and a double-crystal monochromator will be used to divide the original neutron beam into two, and neutron guides will be used to preserve flux and reduce backgrounds [6]. One of the resulting neutron beams will be devoted to experiments requiring cold neutrons $(\mathrm{CN})$ and the other will provide neutrons with a wavelength of $0.89 \mathrm{~nm}$, which will be used to make ultracold neutrons (UCN) via a superthermal process in superfluid helium. Both beam lines will have secondary shutters so that they can be operated independently. It is expected that the $\mathrm{CN}$ beam line will be operational by mid-2007 and the UCN beam line by early 2010. When the SNS is operating at full power, the peak neutron intensity will be greater than at any other facility, and the time-averaged neutron fluence will be greater than that at any continuous neutron source in the United States.

The slate of experiments to be run on beam line 13 will be determined with the help of a program advisory committee. Each experiment may take from one to several years to complete. Although the particular experiments have not yet been chosen, it is anticipated that they will fall into three general categories: (i) neutron beta decay experiments to study the nature of the electroweak interaction, to test the unitarity of the Cabibbo-Kobayashi-Moskawa (CKM) matrix, to search for physics beyond the standard model, and to impact theories of nucleosynthesis during the big bang; (ii) studies of the weak 
interaction between hadrons by measuring parity nonconserving effects in simple two-particle systems such as n-p, n-d, and n- $\alpha$; and (iii) studies of the nature of time-reversal noninvariance and the origin of the cosmological baryon asymmetry via experiments to search for a nonzero electric dipole moment of the neutron.

The neutron science program at ORELA is very different from those at the SNS and HFIR. The largest component of the ORELA program is to obtain neutron capture, neutron total, and neutron-induced fission cross-section measurements for the Nuclear Criticality Safety Program (NCSP) [7]. In addition to these measurements, the overall NCSP program encompasses cross-section evaluations, cross-section modeling, integral measurements, nuclear criticality calculations, and evaluations of the impact of crosssection uncertainties on criticality safety margins. Recent ORELA measurements have shown that there are many problems with older cross-section data that have been used in criticality safety applications. For example, some of the old data were measured with energy resolution too poor for accurate selfshielding correction, had too restricted an energy range, or had large systematic errors due to undercorrected backgrounds or other problems such as missing resonances or resonances assigned to the wrong isotope.

Similar cross-section measurements are needed for other areas of applied nuclear physics such as programs studying the disposal and transmutation of nuclear waste and the next generation of nuclear reactors. It is anticipated that there will be many measurements at ORELA for these programs in the future.

Another component of the ORELA program is cross-section measurements for nuclear astrophysics [8]. These measurements are used to determine the rates of nuclear reactions needed to test and improve models of the big bang, stars, supernovae, and the chemical evolution of the galaxy as well as to obtain improved estimates of the age of the universe. Recent advances in astronomical observations, improvements and changes in astrophysical models (driven by ever faster and larger computers), and new nuclear physics measurement techniques have resulted in the need for more, new, and improved cross-section measurements. For example, recently it has been shown that making (n, $\alpha)$ measurements on intermediate-weight nuclides could be perhaps the best method for improving the rates of $(\gamma, \alpha)$ reactions needed for explosive nucleosynthesis calculations [9]. The rates for these reactions are very difficult or impossible to determine directly using current techniques, and the nuclear model used to calculate these rates is not very well constrained and hence has been unreliable. The first $(n, \alpha)$ measurements have demonstrated that they should be very useful for improving the nuclear model, but, as shown in Figure 1, have turned up some surprises [10]. More measurements of this type are needed to improve the accuracy of $(\gamma, \alpha)$ rates across the wide range of masses needed by the astrophysical models. 


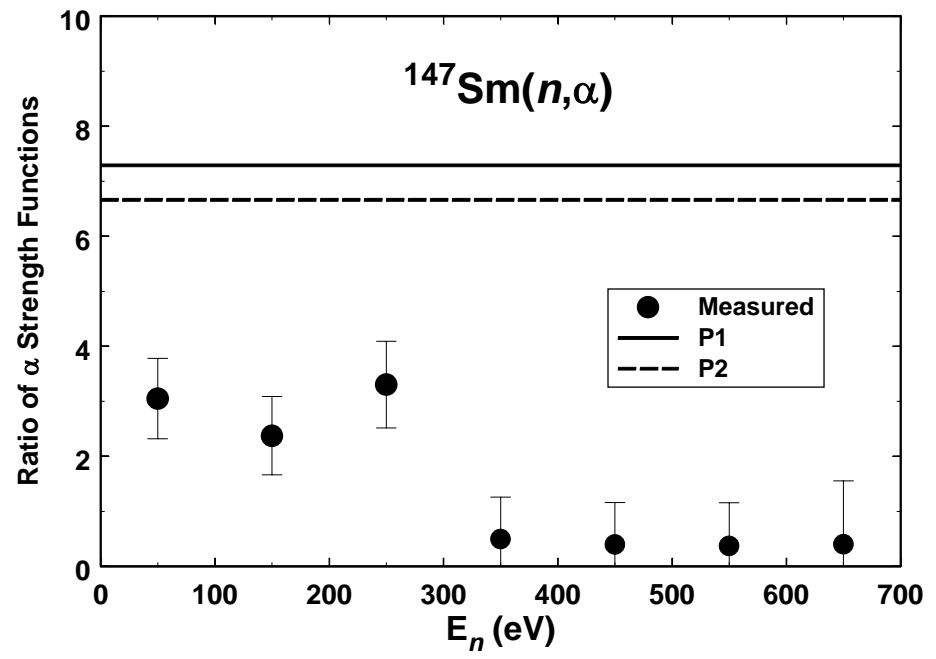

Figure 1. Ratio of $\alpha$ strength functions for 3 to 4 resonances populated in the ${ }^{147} \mathrm{Sm}(n, \alpha)$ reaction [10]. The circles with onestandard-deviation error bars were obtained from a resonance analysis of cross-section measurements at ORELA. The two curves labeled "P1" and "P2" show the expected ratios calculated using the nuclear statistical model with two different input $\alpha$-nucleus potentials. As can be seen, the statistical model calculations overpredict the measured ratio by more than a factor of two. However, even more worrisome is the abrupt change in the measured ratio at about $300 \mathrm{eV}$. Such an abrupt change cannot be calculated using a nuclear statistical model and currently is not understood. More measurements are needed to ascertain if these data are indeed correct as well as for other nuclides.

Excellent resolution across a wide energy range is a crucial feature of ORELA that makes it possible to obtain the high-accuracy cross-section measurements needed for today's applications. This high resolution is made possible by the short pulse width of the electron beam from the ORELA accelerator, the compact, under-moderated design of the neutron production target, and the availability of long flight paths. The price you pay for high resolution is reduced flux. At the SNS, the resolution is much worse but the flux is much larger. Hence, the capabilities of ORELA and the SNS for cross-section measurements are complementary. At ORELA, it is possible to make high-accuracy cross-section measurements using gram-sized samples while at the SNS it is possible to make measurements using much smaller samples. A workshop to explore possible uses of the SNS for experiments of interest to astrophysics, symmetries, and applied physics was held in spring 2002. Although it is clear from the proceedings of the workshop that a beam line at the SNS would make possible many new experiments in these areas [11], there currently are no plans to construct such a beam line. 


\section{CONCLUSIONS}

Neutron science research at ORNL is dominated by neutron scattering, but there also are world-class programs in isotope production, fundamental neutron physics, and basic and applied nuclear physics. Two large facilities, the HFIR and the SNS, when fully operational, will provide world-leading fluxes of steady-state cold and pulsed thermal neutrons, respectively. A third, smaller facility, ORELA, provides

the complementary capability of unsurpassed high-resolution beams of neutrons in the $\mathrm{eV}$ to $\mathrm{MeV}$ energy range.

\section{REFERENCES}

1. $\quad$ http://web.ornl.gov/sci/rrd/pages/hfir.html.

2. R. W. Peele, J. A. Harvey, F. C. Maienschein, L. W. Weston, D. K. Olsen, D. C. Larson, and R. L. Macklin, Technical Report No. ORNL/TM-8225, Oak Ridge National Laboratory, unpublished; http://www.phy.ornl.gov/nuclear/orela/.

3. Technical Report No. SNS 100000000-PL0001-R13; http://www.sns.gov/aboutsns/baseline.htm.

4. http://www.sns.gov/documentation/Basic_Fact_Sheet.pdf.

5. $\quad$ http://web.ornl.gov/sci/rrd/pages/brochure.pdf.

6. P. R. Huffman, G. L. Greene, R. R. Allen, V. Cianciolo, R. R. Huerto, P. Koehler, D. Desai, R. Mahurin, A. Yue, G. R. Palmquist, and W. M. Snow, J. Res. Natl. Inst. Stand. Technol. 110, 161 (2005).

7. K. Guber, contribution to these proceedings.

8. http://www.phy.ornl.gov/astrophysics/nuc/neutrons/whitepaper.pdf.

9. Yu. M. Gledenov, P. E. Koehler, J. Andrzejewski, K. H. Guber, and T. Rauscher, Phys. Rev. C 62, 042801(R) (2000).

10. P. E. Koehler, Yu. M. Gledenov, T. Rauscher, and C. Frohlich, Phys. Rev. C 69, 015803 (2004).

11. Astrophysics, Symmetries, and Applied Physics at Spallation Neutron Sources, edited by P. E. Koehler, R. C. Haight, C. R. Gould, and T. E. Valentine (World Scientific, Singapore, 2002). 


\title{
Neutron Cross-Section Measurements at ORELA
}

\author{
K. H. Guber, L. C. Leal, R. O. Sayer, P. E. Koehler, H. Derrien, D. Wiarda, and J. A. Harvey \\ Oak Ridge National Laboratory, ${ }^{1}$ P.O. Box 2008, Oak Ridge, TN 37831-6354, USA
}

\begin{abstract}
The Oak Ridge Electron Linear Accelerator (ORELA) is the only highpower white neutron source with excellent time resolution still operating in the United States and is ideally suited to measure fission, neutron total, and capture cross sections. For many nuclear criticality safety applications in the important neutron energy range from $1 \mathrm{eV}$ to $\sim 600 \mathrm{keV}$, many of the neutron cross sections from data libraries such as ENDF/B-VI or JENDL-3.2 exhibit large deficiencies. These deficiencies may occur in the resolved and unresolved-resonance regions. Consequently, these evaluated data may not be adequate for nuclear criticality calculations where effects such as self-shielding, multiple scattering, or Doppler broadening are important. To support the Nuclear Criticality Predictability Program, neutron cross-section measurements have been initiated at ORELA.
\end{abstract}

\section{INTRODUCTION}

For the last several years, concerns about existing nuclear data have been the prime motivator for new cross-section measurements at the Oak Ridge Electron Linear Accelerator (ORELA). Many older neutron cross-section evaluations from libraries such as ENDF/B-VI or JENDL-3.2 exhibit deficiencies or do not cover energy ranges that are important for criticality safety applications. Because many of the older evaluations were derived from measurements made with poor time-of-flight (TOF) resolution, the description of some data in the neutron energy range above several tens of $\mathrm{keV}$ is crude. Deficiencies may occur in the resolved- and unresolved-resonance regions. Therefore, some of these evaluated data may not be adequate for criticality calculations where effects such as self-shielding, multiple scattering, or Doppler broadening are important. Furthermore, many evaluations for nuclides having small neutron capture cross sections are erroneously large. Although their neutron capture cross sections are small, these nuclides can be important absorbers in many criticality calculations, and accurate cross-section data are essential. Of the several neutron sources in the United States, ORELA is the only operating highpower white neutron source with excellent time resolution in the energy range from thermal to about $1 \mathrm{MeV}$. Therefore, ORELA is ideally suited for measuring fission, neutron total, and capture cross sections in the energy range from $1 \mathrm{eV}$ to $\sim 600 \mathrm{keV}$, which is important for nuclear criticality safety applications. In fact, over 180 isotopes found in the evaluated libraries originated from ORELA crosssection measurements.

\footnotetext{
${ }^{1}$ Managed by UT-Battelle, LLC, under contract DE-AC05-00OR22725 with the U.S. Department of Energy.

The submitted manuscript has been authored by a contractor of the U.S. Government under contract No. DE-AC05-00OR22725. Accordingly, the U.S. Government retains a nonexclusive, royalty-free license to publish or reproduce the published form of this contribution, or allow others to do so, for U.S. Government purposes.
} 


\section{WHITE NEUTRON SOURCES IN THE UNITED STATES}

There are several white neutron sources still operating in the United States. Among them are the Los Alamos Neutron Scattering Science Center (LANSCE) facility at the Los Alamos National Laboratory (LANL) with the Lujan center and the Weapons Neutron Research Facility (WNR), the Intense Pulsed Neutron Source (IPNS) at the Argonne National Laboratory (ANL), the Gaerttner Linear Accelerator at Rensselaer Polytechnic Institute (RPI), and ORELA. LANSCE and IPNS are spallation neutron sources driven by proton accelerators, whereas the RPI linac and ORELA are electronaccelerator-driven neutron sources. Each facility has its own characteristics, depending on the design of the accelerator and the neutron production target. For example, the Lujan center and IPNS are optimized for a high neutron flux in the thermal neutron energy range, which requires rather large moderators and broad pulses of the primary protons. On the other hand, the electron-linac-driven sources use smaller neutron target moderator assemblies and have shorter, variable-width pulses. Two important parameters define the quality of a neutron source and are used for classification. First, there is the neutron flux and spectrum, which should be suitable for the desired cross section measurement of the isotope of interest. Second, there is the neutron energy resolution, which defines how well individual resonances in the cross section can be distinguished from one another. High resolution can be of major importance, even if only the unresolved or average cross-section region is of primary interest, because average cross sections for applications can most reliably be determined from average resonance parameters resulting from the analysis of high-resolution measurements.

One useful figure of merit for comparing facilities is the neutron flux divided by the square of the resolution as a function of neutron energy. Relevant facility parameters are compiled in Table 1. In Figure 1, the figure of merit for three facilities at given flight path lengths and primary beam characteristics are plotted for comparison.

Table 1. U.S. white neutron source parameters for the intermediate energy range

\begin{tabular}{lcccc}
\hline \multicolumn{1}{c}{ Facility parameters } & ORELA & LANSCE/Lujan & IPNS & RPI \\
\hline Sources & $\mathrm{e}^{-}$linac & p spallation & p spallation & $\mathrm{e}^{-}$linac \\
Particle E (MeV) & 140 & 800 & 450 & $>60$ \\
Flight path (m) & $10-200$ & $7-55$ & $\sim 6-20$ & $10-250$ \\
Pulse width (ns) & $2-30$ & 125 & 40 & $15-5000$ \\
Max power (kW) & 50 & 100 & 6.3 & $>10$ \\
Rep rate (Hz) & $1-1000$ & 20 & 30 & $1-500$ \\
Intrinsic resolution (ns/m) & 0.01 & 3.9 & 2.0 & 0.06 \\
Neutrons/s & $1 \times 10^{14}$ & $1.2 \times 10^{16}$ & $8.1 \times 10^{14}$ & $4 \times 10^{13}$ \\
\hline
\end{tabular}




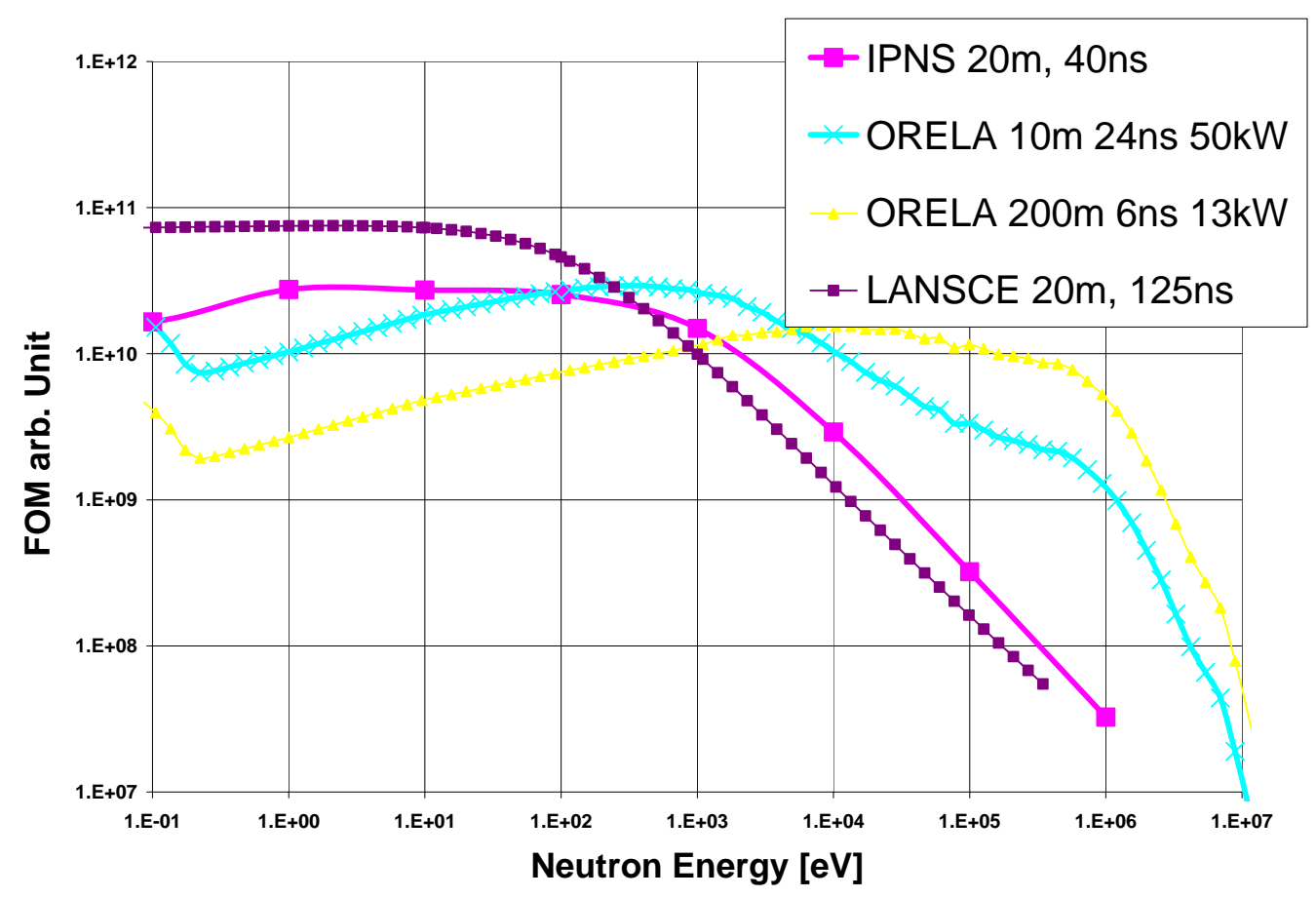

Figure 1. Comparison of figures of merit (neutron flux divided by the square of the resolution as a function of neutron energy) for the different neutron sources in the United States. In the case of LANSCE, the neutron flux was taken for the flight path of the Detector for Advanced Neutron Capture Experiments.

\section{NUCLEAR DATA}

Design and analysis codes for nuclear systems rely on evaluated cross sections and covariances from nuclear data libraries. These libraries, in turn, are built from cross-section measurements. Therefore, high-quality nuclear cross-section measurements are required for efficiency and safety analyses of new GEN-IV reactor designs; nuclear waste storage, transportation, and transmutation; and accelerator-driven subcritical systems.

Problems with existing nuclear data have emerged over the past few years, such as improper pulse-height weighting functions, neutron sensitivity backgrounds, poorly characterized samples, poor TOF resolution, and restricted energy ranges. Furthermore, corrigenda were published after discovering errors in the computer data reduction code (the correction factors ranged from 0.7480 to 1.1131 for 46 nuclides from ${ }^{24} \mathrm{Mg}$ to ${ }^{232} \mathrm{Th}$ [1] and from 0.9507 to 1.208 for 47 nuclides from ${ }^{23} \mathrm{Na}$ to ${ }^{206} \mathrm{~Pb}$ [2]).

The validity of the calculated pulse-height weighting function used in the neutron capture experiments was questioned after a $20 \%$ discrepancy was found in the neutron width of $1.15-\mathrm{keV}$ resonance in ${ }^{56} \mathrm{Fe}$. Corvi et al. [3] overcame this problem by using an experimentally determined weighting function. On the other hand, using the Monte Carlo code EGS4 [4], Perey et al. [5] showed that a careful calculation of the weighting function also could resolve this problem. 
The neutron sensitivity background appears to have been incorrectly accounted for in some of the old neutron capture data. This background is caused by neutrons scattered from the sample and subsequently captured in the detector or surroundings within the time corresponding to the width of the resonance. It has led to erroneously large capture areas in the old data for resonances having large neutron widths, as shown in Figure 2. In those cases it has led to incorrectly large capture kernels in the current ENDF/B-VI evaluations. The new neutron capture apparatus at ORELA has been improved in many ways compared to the old apparatus with the result that this neutron sensitivity background has been reduced to the point where it is no longer a problem.

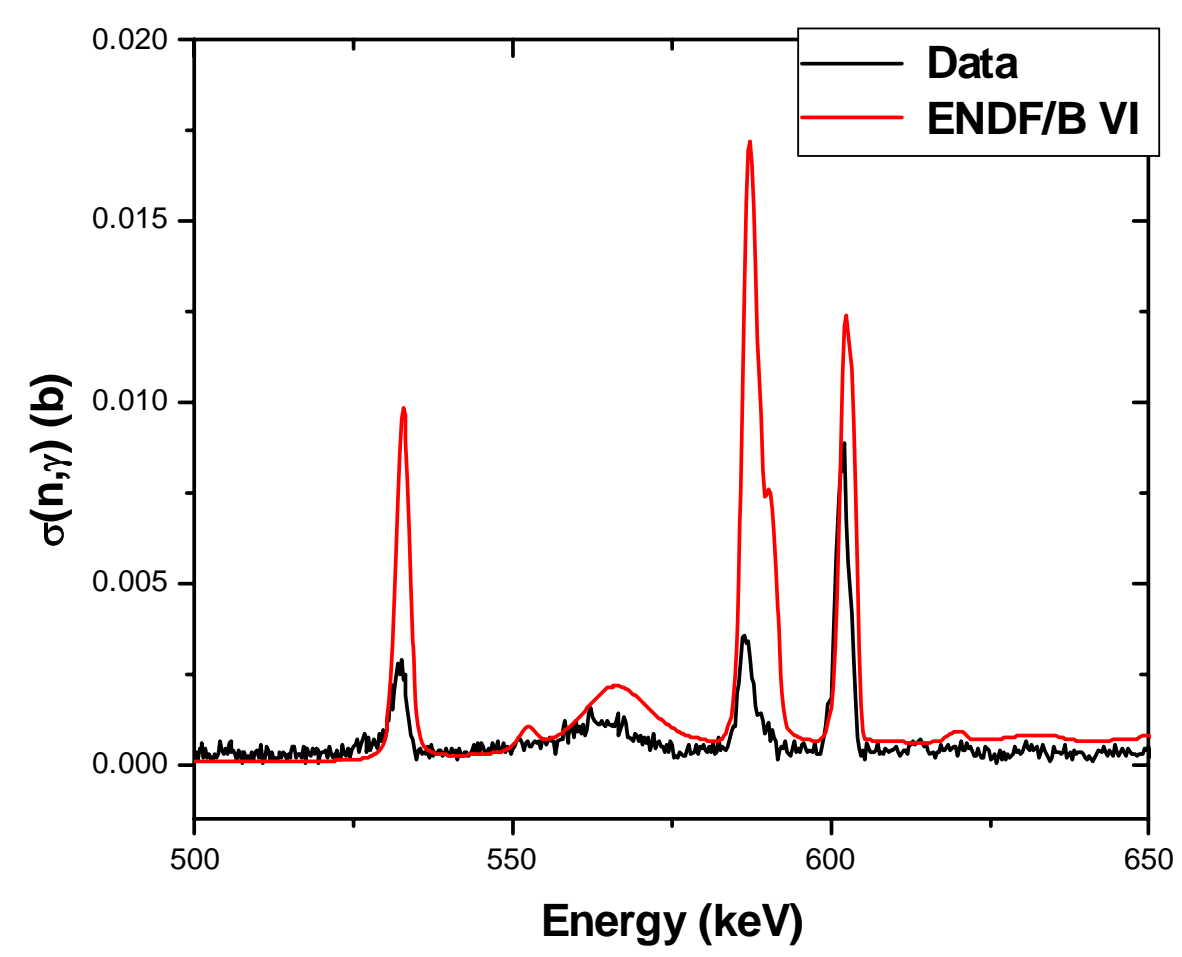

Figure 2. The large neutron sensitivity of older measurements led to many erroneously large resonance areas in current evaluations. The black curve represents the new ORELA experimental data. The red curve is the calculated cross section including all experimental effects using the ENDF/BVI evaluation (which is based on the older measurement) for Si.

Although efforts were made to use highly (isotopically) enriched and chemically pure samples, it appears that sometimes problems with a sample's chemical composition led to large systematic errors in some measurements. For example, some samples were available only as oxides, which can be hygroscopic and therefore pick up water fairly easily. A substantial water content in the sample can lead (via moderation effects) to erroneously large cross sections.

Because of computer storage system limitations, many of the older cross-section measurements were performed with data bins that were too coarse. As a consequence, those data sets sometimes have too few data points over narrow resonances to accurately calculate corrections for experimental effects such 
as Doppler broadening, self-shielding, and multiple scattering. In addition, many of the older experiments were run with a low-energy cut-off of around $3 \mathrm{keV}$. However, this missing energy range can be important for current nuclear criticality calculations. Hence, new measurements are needed in these cases.

\section{EXPERIMENTAL SETUP AT ORELA}

ORELA consists of a 180-MeV electron linear accelerator, a neutron-producing target, underground and evacuated flight tubes, sophisticated detectors, and data acquisition systems. The accelerator is highly flexible because of its variable repetition rate $(1-1000 \mathrm{~Hz})$ and burst width (2-30 ns). At full power, the average neutron flux is $10^{14}$ neutrons/s. Simultaneous measurements are possible at 18 detector stations on 10 separate flight paths at distances between 9 and $200 \mathrm{~m}$ from the neutron source. The TOF technique has been used in the energy range from thermal up to $50 \mathrm{MeV}$ to measure neutron total, capture, fission, elastic, inelastic, (n, $\alpha$ ), and $\gamma$-ray and neutron production cross sections.

\section{Capture Measurements}

Neutron capture experiments at ORELA are usually performed at the 40-m flight station, on flight path 7. A pair of deuterated benzene $\left(\mathrm{C}_{6} \mathrm{D}_{6}\right)$ detectors is used to detect the capture $\gamma$-rays; the pulse-heightweighting method is applied. Over the last couple of years, the system has been improved in many ways compared to the old ORELA apparatus [6]: First, most of the structural material surrounding the sample and detectors was reduced to decrease the background from sample-scattered neutrons (neutron sensitivity background). This was accomplished by replacing the massive $\mathrm{Al}$ sample changer and beam pipe with a thin carbon fiber tube. The steel detector housings were replaced with reduced-mass detector mounts. Second, the $\mathrm{C}_{6} \mathrm{~F}_{6}$ scintillator was replaced with a $\mathrm{C}_{6} \mathrm{D}_{6}$ scintillator, which has much lower neutron sensitivity. More details about these improvements can be found in the papers by Koehler et al. [7, 8]. For two resonances in ${ }^{88} \mathrm{Sr}$, at 289 and $325 \mathrm{keV}$ with neutron widths $\mathrm{g} \Gamma_{\mathrm{n}}=24,932$ and 22,082 eV, respectively, the measured capture widths were an average factor of five smaller than reported from measurements using the old system (after a correction for neutron sensitivity already had been applied to the old data). Third, calculation of the detector weighting functions has been improved by using the Monte Carlo code EGS4 and by including the sample and all structural materials within $30 \mathrm{~cm}$ of the detectors in the calculations.

Over the last few years, we used the new setup to perform several neutron capture cross-section experiments on elements with small capture cross sections ( $\mathrm{Al}, \mathrm{Cl}, \mathrm{F}, \mathrm{Si}$, and $\mathrm{K}$ ) that are of interest for nuclear criticality. A 1.27-cm-thick $\mathrm{Pb}$ filter was employed to reduce the $\gamma$-ray background from the neutron production target; and pulse overlap neutrons were eliminated with a $0.48-\mathrm{g} / \mathrm{cm}^{2}{ }^{10} \mathrm{~B}$ filter. Absolute cross sections were determined by using the saturated resonance technique, employing the 4.9-eV resonance in gold [9]. A 0.5-mm-thick ${ }^{6} \mathrm{Li}$-glass scintillator, placed $42.1 \mathrm{~cm}$ upstream of the sample position, was used to measure the energy dependence of the neutron flux. 


\section{Transmission Measurements}

High-resolution transmission experiments for determining the total cross sections are not only indispensable for evaluations but are also necessary for calculating self-shielding and multiple-scattering corrections for neutron capture cross-section measurements. Given the fact that capture experiments cannot be performed with an infinitesimally thin sample (in fact, sometimes the samples are quite thick), these corrections can be sizeable. Consequently, we made corresponding total cross-section measurements when needed. Also, transmission measurements sometimes can be more sensitive to certain resonances than $(n, \gamma)$ measurements. The neutron beam was collimated to about $2.54 \mathrm{~cm}$ on the samples and allowed only neutrons from the water moderator part of the neutron source to be used. The neutron detector was an 11.1-cm-diameter, 1.25 -cm-thick ${ }^{6} \mathrm{Li}$-glass scintillator positioned in the beam at a distance of $79.815 \mathrm{~m}$ from the neutron source. For background reduction, the scintillator was viewed edge-on by two 12.7-cm-diameter photomultipliers that were placed outside the neutron beam. To reduce systematic uncertainties, we cycled the samples and their compensators or corresponding empty containers periodically through the neutron beam; the neutron flux was recorded for each sample and cycle. Additional measurements with a thick polyethylene sample were used to determine the $\gamma$-ray background from the neutron source.

\section{EXPERIMENTAL RESULTS}

We found significant differences between our new capture and transmission data and the evaluations for $\mathrm{Al}, \mathrm{Cl}, \mathrm{F}, \mathrm{K}$, and Si obtained from the ENDF/B-VI or JENDL-3.2 nuclear data libraries. For these elements with small capture cross sections, our new results are even smaller than the previous ones. It is evident from our new data that in many previous cases, the capture widths were severely overestimated and resonances were missed as a result of large backgrounds. The new high-resolution total crosssection measurements reveal previously misassigned resonances and enabled us to extend the resolved resonance region to much higher energies. An example of the data is given in Figure 3, where we plotted the transmission data for natural metallic potassium and the corresponding capture data from our $\mathrm{K}_{2} \mathrm{CO}_{3}$ sample.

The discrepancies observed between our data and the evaluated data from the nuclear data libraries have two main causes. First, the use of improper weighting functions resulted in mismatched detector response functions. Second, underestimated neutron sensitivity of the experimental setups resulted in previous capture cross sections that were too large. Together with the better characterized samples, the superior TOF resolution, and the well-understood experimental setups and backgrounds, the new experimental data enable us to produce more reliable resonance parameters.

The neutron total and capture cross-section data were analyzed with SAMMY [10]. This code made the necessary corrections for the experimental effects, such as Doppler and resolution broadening, selfshielding, and multiple-scattering effects. The new resonance parameters were then used as a starting point for an evaluation. In this evaluation other existing experimental data sets were included whenever they were available and suitable. The new cross-section data set was then used for criticality benchmark calculations and was checked for inconsistencies. As an example, the ORNL evaluation for Si shows large discrepancies from capture cross sections found in the ENDF/B-VI nuclear data library. We observed two resonances for ${ }^{28} \mathrm{Si}$ that had not been previously reported. In addition, we determined that one resonance previously assigned to ${ }^{28} \mathrm{Si}$ is actually in ${ }^{30} \mathrm{Si}$. Furthermore, a reported resonance in ${ }^{30} \mathrm{Si}$ at $2.235 \mathrm{keV}$ was not visible in our new capture data and was not in transmission measurements of an enriched ${ }^{30} \mathrm{Si}$ sample. The result of this evaluation [11] is shown in Figure 4, where the unbroadened capture cross sections are plotted. For the correct capture of Si, the direct capture component has to be added to the resonant capture of the ORNL evaluation. 
In the case of $\mathrm{Cl}$ we found similar results [12]. The neutron capture cross section is too large in the ENDF/B-VI evaluation, and the resolved energy range is very limited compared with our data (Figure 5).
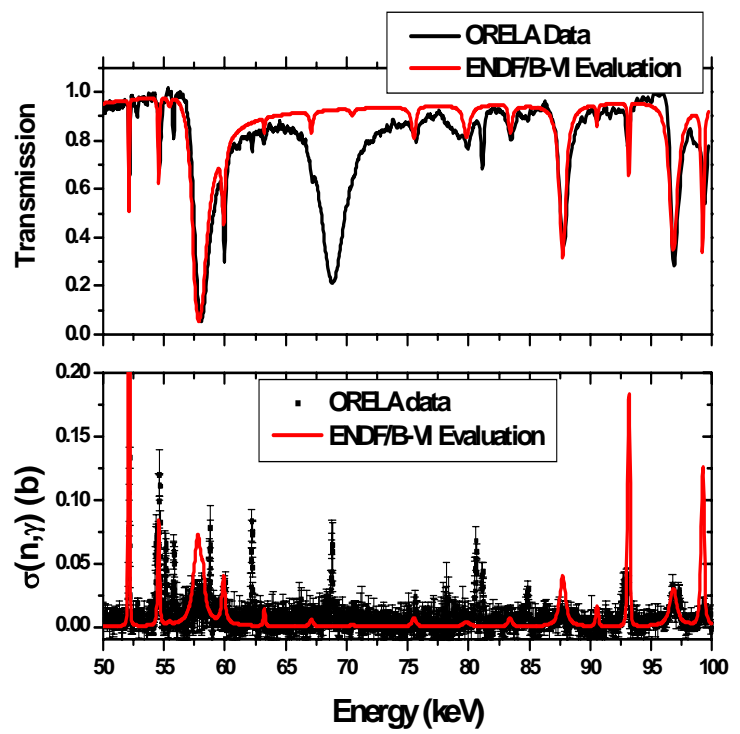

Figure 3. Transmission and capture of natural potassium compared with the transmission and capture calculated from JENDL3.2 parameters. The fact that the strong resonance at $68.8 \mathrm{keV}$ in transmission is not reported seem to be puzzling and could be very well a misprint in JENDL.

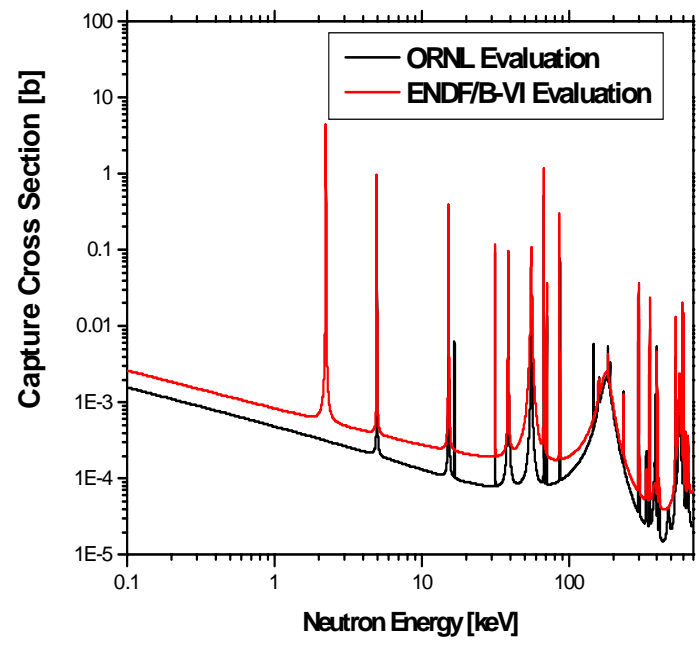

Figure 4. Evaluations of natural Si from ENDF/B-VI (red curve) compared with the ORNL evaluation (black curve). To obtain the correct ORNL neutron capture, the contribution of the direct capture calculation must be added to the resonant capture from the ORNL evaluation. 


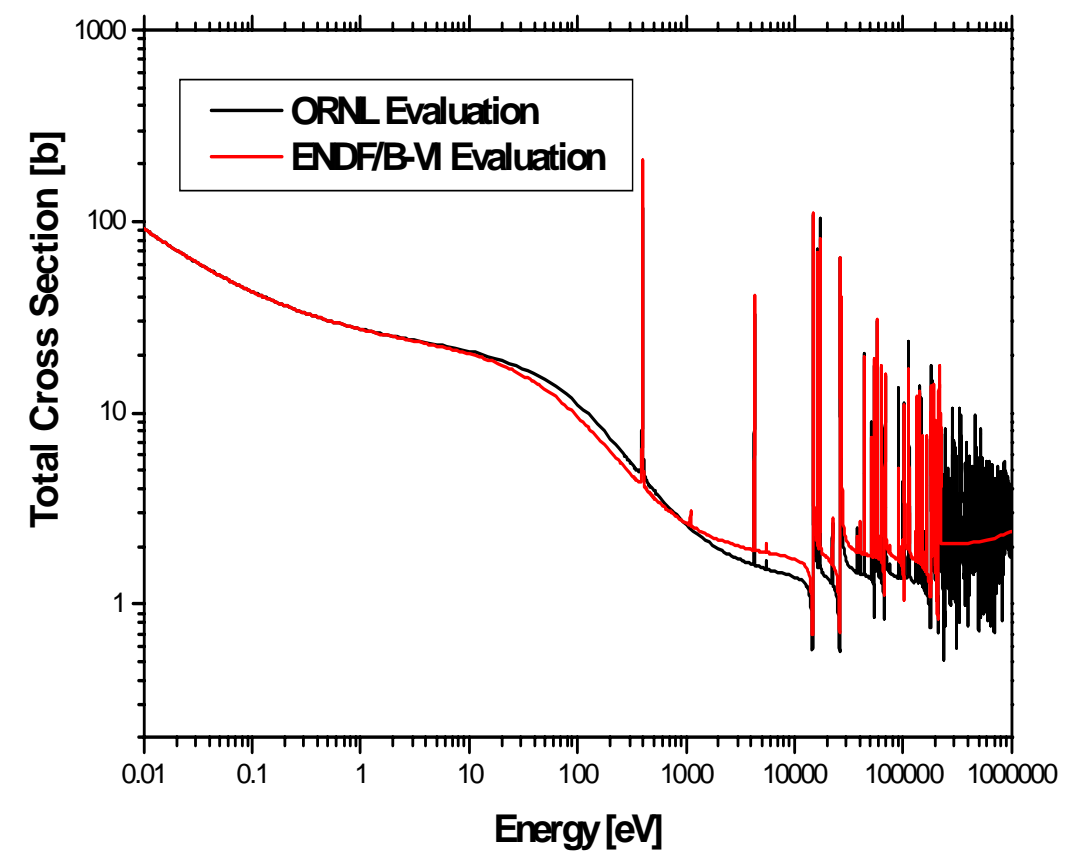

Figure 5. Evaluations of Cl from ENDF/B-VI (red curve) compared with the ORNL evaluation (black curve).

\section{CONCLUSIONS}

To support the Nuclear Criticality Safety Program, we performed new neutron total and capture measurements at ORELA over broad energy ranges. We then used the SAMMY multilevel R-matrix code. In all analyzed and evaluated cases, we were able to extend the resolved resonance region to much higher energies than the existing evaluations. These new evaluations should lead to much more reliable nuclear criticality calculations.

We would like to emphasize one particular finding. Over the past ten years, the results of our new neutron capture cross-section measurements at ORELA for samples with large scattering cross sections have shown the tendency to be smaller than the data found in the nuclear data libraries. Therefore, many of the older measurements for samples with small capture cross sections are questionable, or at least much more uncertain, especially if the applied corrections for neutron sensitivity were sizeable.

\section{ACKNOWLEDGMENTS}

ORNL is managed by UT-Battelle, LLC, for the U.S. Department of Energy under Contract No. DEAC05-00OR22725. The work that is presented in this paper was sponsored by the U.S. Department of Energy Nuclear Criticality Safety Program. 


\section{REFERENCES}

1. $\quad$ R. L. Macklin, and R. R. Winters, Nucl. Sci. Eng. 78, 110-111 (1981).

2. $\quad$ B. J. Allen, J. W. Boldeman, and R. L. Macklin, Nucl. Sci. Eng. 82, 230-231 (1982).

3. F. Corvi, A. Prevignano, and H. Liskien, Nucl. Instrum. Methods A265, 475-484 (1988).

4. W. R. Nelson, H. Hirayama, and D. W. O. Rogers, The EGS4 Code System, Report SLAC-265, 1985.

5. F. G. Perey, J. O. Johnson, T. A. Gabriel, R. L. Macklin, R. R. Winters, J. H. Todd, and N. W. Hill, in Nuclear Data for Science and Technology, edited by S. Igarasi, JAERI Conference Proceedings, Mito, Japan, May 30-June 3, 1988, JAERI Conference Proceedings, pp. 379-382.

6. $\quad$ R. L. Macklin and B. J. Allen, Nucl. Instrum. Methods, 91, 565 (1971).

7. P. E. Koehler, R. R. Spencer, R. R. Winters, K. H. Guber, J. A. Harvey, N. W. Hill, and M. S. Smith, Phys. Rev. C, 54, 1463-1477 (1996).

8. P. E. Koehler, R. R. Winters, K. H. Guber, T. Rauscher, J. A. Harvey, S. Raman, R R. Spencer, J. C. Blackmon, D. C. Larson, D. W. Bardanyan, and T. A. Lewis, Phys. Rev. C, 62, 055803-1 (2000).

9. $\quad$ R. L. Macklin, J. Halperin, and R. R. Winters, Nucl. Instrum. Methods, 164, 213 (1979).

10. N. M. Larson, ORNL/TM-9179/R6, Oak Ridge National Laboratory, 2003.

11. H. Derrien, L. C. Leal, K. H. Guber, T. E. Valentine, N. M. Larson, and T. Rauscher, ORNL/TM2001/271, Oak Ridge National Laboratory, 2002.

12. R. O. Sayer, K. H. Guber, L. C. Leal, and N. M. Larson, in Proceedings of the International Conference on Nuclear Data for Science and Technology, Santa Fe (2004), p. 386. 


\title{
LANSCE and ORELA Complementary Measurement Capabilities
}

\author{
Robert C. Haight \\ Los Alamos Neutron Science Center, Los Alamos National Laboratory \\ Los Alamos, NM 87545
}

\begin{abstract}
The complementary capabilities of the LANSCE and ORELA facilities for making measurements of neutron-induced reaction cross sections are summarized. The capabilities are complementary in the neutron source characteristics, the instruments available on the flight paths, and the missions of the two facilities. Some of the present measurements under way at LANSCE are summarized.
\end{abstract}

\section{INTRODUCTION}

LANSCE and ORELA have been used now for many years for nuclear physics experiments and nuclear data measurements for applied programs. This view of their complementary capabilities concentrates on these two pulsed, "white" (continuous in energy) neutron sources. Other white sources in the United States at the Intense Pulsed Neutron Source (IPNS) at Argonne National Laboratory and the Gaertner Laboratory Linear Accelerator at Rensselaer Polytechnic Institute, are described by others a this meeting. Other pulsed neutron sources are at the Institute for Reference Measurements and Materials at Geel, Belgium; at n_TOF at CERN; and at laboratories in Russia, Japan, and other locations.

LANSCE and ORELA have had for many years a close cooperation, driven in large measure by the common interest in neutron energy and defense programs. In the 1970s, for example, an LANL-ORNL collaboration at ORELA led by Keyworth et al. [1] made pioneering measurements of the spins of fission resonances using polarized targets of actinides. These data and others form the experimental database for evaluations of neutron-nuclear data for ${ }^{235} U$ [2] and other nuclides. In the area of gamma-ray production, this author was fortunate to work with J. K. Dickens to evaluate neutron-induced gamma production on tantalum. More recently, D. W. Larson of ORELA came to LANSCE to measure the gamma-ray production on iron [3]. In the field of technology, LANSCE has benefited from hardware developed at ORELA, e.g., by N. W Hill [4].

\section{SOURCE CHARACTERISTICS}

Both LANSCE and ORELA produce neutrons by pulsed, charged-particle beams incident on heavy metal targets, either $800-\mathrm{MeV}$ proton beams on tungsten (LANSCE) or by $140-\mathrm{MeV}$ electron beams on tantalum or tantalum-beryllium (ORELA). The characteristics of the ORELA source are described elsewhere in these proceedings. These sources have been compared previously by Michaudon and Wender [5] and, for neutron capture experiments on small samples, by Koehler [6]. The information presented below updates the former and the description by Lisowski et al. [7] by the increased beam current now available at LANSCE.

LANSCE has in fact four neutron sources:

- fast neutrons from 0.1 to $800 \mathrm{MeV}$ at the Weapons Nuclear Research Facility, where the effective upper limit is closer to $600 \mathrm{MeV}$; 
- moderated neutrons at the Manuel Lujan Jr. Neutron Scattering Center (usually referred to as the "Lujan Center" or "MLNSC") with usable neutron energies from cold neutrons to $200 \mathrm{keV}$ or so;

- lead slowing-down spectrometer (LSDS), where the neutron energies span the range of $0.3 \mathrm{eV}$ to $200 \mathrm{keV}$;

- ultracold neutron source with neutrons of a few neV in energy - this will not be described further in this report, as the source is under development and there is no comparable source at ORELA.

Thus LANSCE can provide neutrons with energies over 16 orders of magnitude in energy.

Typical neutron output spectra from the Lujan and WNR neutron sources are given in Figure 1, where they are compared with those from ORELA. I refer to these spectra as "typical" because they are the most-used spectra at the two facilities. The Lujan spectrum [8] comes from the "partially decoupled" upper-tier moderator that is used with the Detector for Advanced Neutron Capture Experiments (DANCE). The WNR spectrum [9] is that at 30-degrees production angle, which, for the highest neutron energies, has somewhat fewer neutrons than that at the 15-degree production angle, but more than at 60 and 90 degrees. Conversely, this 30-degree angle has somewhat more neutrons below $5 \mathrm{MeV}$ than at 15 degrees but less than at 60 or 90 degrees. Details are given on the WNR web page [10]. The ORELA spectra are given for a variety of targets: moderated tantalum, beryllium, and unmoderated tantalum targets, all at $50 \mathrm{~kW}$ electron beam power [5]. The LSDS output, which has very poor energy resolution, is that produced by $1 \mu \mathrm{A}$ of the 800 proton beam on a tungsten target at a typical location in the lead assembly $[11,12]$.

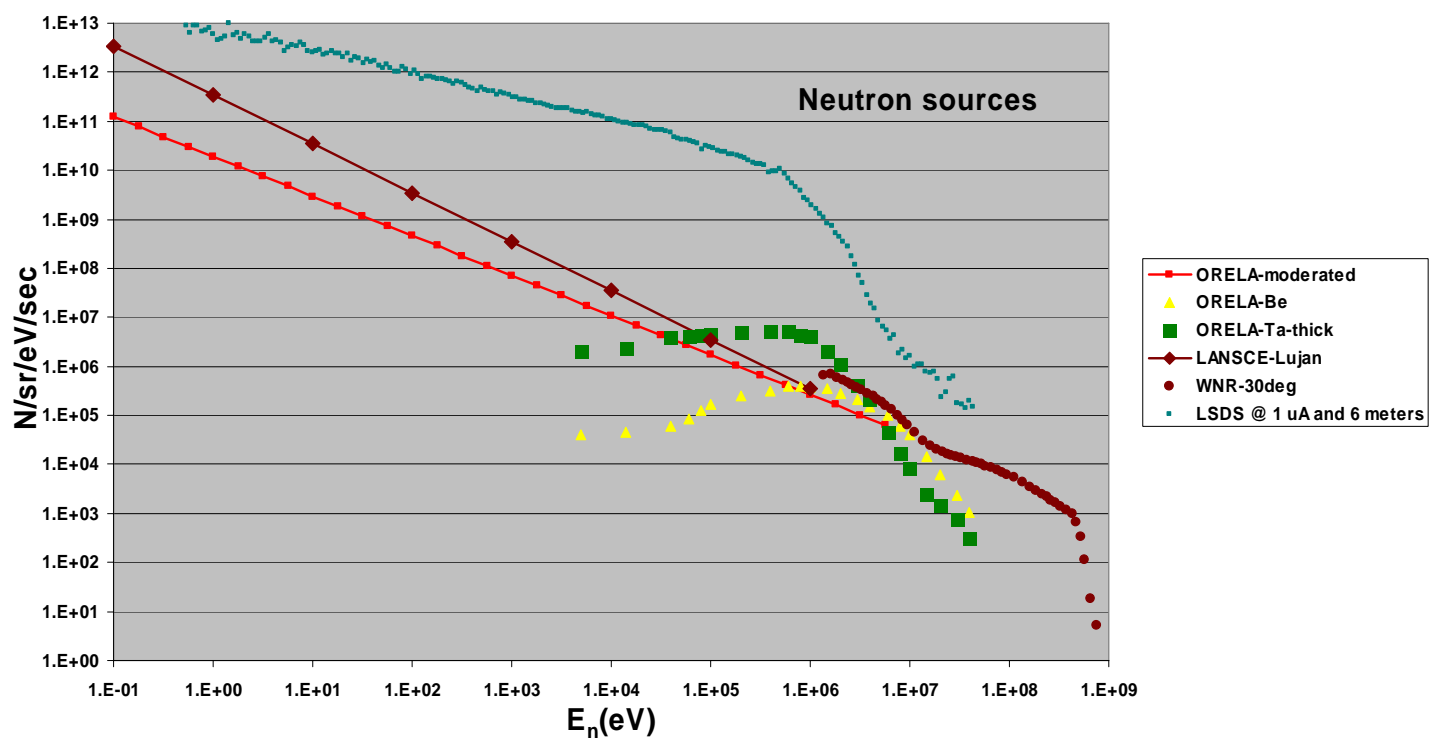

Figure 1. Neutron source spectra from LANSCE and ORELA. The LANSCELujan spectrum is from measurements at Flight Path 14 with $100 \mu \mathrm{A}$ on target. That of WNR-30deg is the 30-degree flight path at LANSCE/WNR with $4 \mu \mathrm{A}$ on target. The LSDS results were transformed from neutrons $/ \mathrm{cm}^{2}$ to neutrons/sr, assuming an equivalent source-sample distance of 6 meters. The spectra from ORELA were reported in Ref. [5]. The beryllium and tantalum targets were assumed to be 20 radiation lengths thick. 
One can see from this comparison of neutron outputs that ORELA can produce more neutrons in the $50 \mathrm{keV}$ to $1 \mathrm{MeV}$ range than the Lujan and WNR sources, with LANSCE producing more neutrons in range below $50 \mathrm{keV}$ and above $2 \mathrm{MeV}$. Neutrons above $\sim 60 \mathrm{MeV}$ are not available at ORELA.

The time resolution determined by the beam pulse width is $135 \mathrm{~ns}$ (FWHM) at the Lujan Center and $\sim 200$ ps at WNR. The resolution of the LSDS is $\sim 30 \%$ in DE/E over most of the neutron energy range. ORELA quotes a resolution of 2 to $30 \mathrm{~ns}$. Both WNR and ORELA use very undermoderated targets, whereas Lujan's are overmoderated.

Flight paths for doing experiments at LANSCE have been described previously [7].

\section{INSTRUMENTS}

Key to doing experiments is the set of specific instruments available at these facilities. Again, I leave to others a description of the instruments at ORELA. LANSCE has a suite of instruments that have been constructed over the last 10 to 15 years by a steady program of investment. The major instruments at LANSCE have been described in the references and include:

GEANIE - GErmanium Array for Neutron Induced Excitations is an array of 26 high-purity germanium (HPGe) detectors [13]. This array originated at the Lawrence Berkeley Laboratory as the HERA array. It has undergone extensive modifications since then.

FIGARO - Fast neutron-Induced Gamma-Ray Observer is an array of 20 liquid scintillator neutron detectors; the event is triggered by a pulse from a fission chamber at the center of the array or from a gamma ray emitted in the reaction $[14,15]$.

$\mathbf{N}, \mathbf{Z}$ - a small array of charged-particle detectors, each consisting of a gas proportional counter, a silicon surface barrier detector, and a stopping $\mathrm{CsI}(\mathrm{Tl})$ detector [16].

DANCE - Detector for Advance Neutron Capture Experiments is a 4-pi array of $\mathrm{BaF}_{2}$ detectors that serve as a calorimeter to measure the total energy emitted as gamma rays following a neutron capture reaction $[17,18]$.

LSDS - a 20-ton Lead Slowing-Down Spectrometer for measuring neutron-induced fission and (n,alpha) reactions is on loan from the CEA laboratory at Bruyères-le-Châtel, France [11, 12].

Fission - a Frisch grid ion chamber for studing fission is being developed in collaboration with the Institute for Reference Measurements and Materials in Belgium [19].

Detectors for total cross section measurements. The detectors used in the wide ranging measurements of 36 materials for neutron energies from 5 to $50 \mathrm{MeV}$ were designed and fabricated at Ohio University [20, 21].

Thus with this set of instruments, a wide range of experimental studies can be carried out. 


\section{PEOPLE AND MISSION}

Crucial to the experimental program at LANSCE are the collaborations with researchers in other divisions at Los Alamos, with other national laboratories and universities, and with foreign participants. The present collaborations includes scientists from many divisions at Los Alamos and from Lawrence Livermore National Laboratory, Lawrence Berkeley National Laboratory, Oak Ridge National Laboratory, Brookhaven National Laboratory, Idaho National Laboratory, North Carolina State University, Ohio University, Colorado School of Mines, Harvard, University of Kentucky, Georgia Tech, CEA Bruyères-le-Châtel (France), Forschungszentrum Karlsruhe (Germany), Institute for Reference Materials and Measurements (Belgium) and Kyushu University (Japan).

The mission of LANSCE is complementary to that of ORELA. Los Alamos is a laboratory of the National Nuclear Security Administration (NNSA), which supports the operation of the LANSCE accelerator and some of the research projects. Other funding for nuclear physics research at LANSCE comes from the Department of Energy's Office of Nuclear Energy, Science and Technology and the Office of Science, and from internal LANL funds (LDRD)

\section{COMPLEMENTARY CAPABILITIES}

In my view, the capabilities of ORELA and LANSCE can be summarized in the following lists:

\section{ORELA}

- excellent resolution in resonance region

- $\quad$ well-understood source term (essential input for SAMMY)

- neutron flux larger in $\sim 50 \mathrm{keV}$ to a few $\mathrm{MeV}$ region

- many well-characterized instruments

- many flight paths

- $\quad$ ability to do classified experiments

\section{LANSCE}

- $\quad$ excellent timing for fast neutrons $(<1 \mathrm{~ns}$ at WNR)

- $\quad$ high intensity for En $<50 \mathrm{keV}$ (Lujan) and En > a few MeV (WNR)

- energy range extends to $\sim 600 \mathrm{MeV}$

- low gamma-flash for fast neutrons (WNR)

- many well-characterized instruments

- many flight paths — used simultaneously

- ability to access proton beam directly (e.g., LSDS)

- $\quad$ ability to do classified experiments

These capabilities are complementary and present a very useful set of options to the community of experimentalists.

\section{ACKNOWLEDGMENTS}

This work has benefited from the use of the Los Alamos Neutron Science Center at the Los Alamos National Laboratory. This facility is funded by the U.S. Department of Energy and operated by the University of California under Contract W-7405-ENG-36. 


\section{REFERENCES}

1. G. A. Keyworth, J. R. Lemley, C. E. Olsen, F. T. Seibel, J. W. T. Dabbs, and N. W. Hill, Phys. Rev. C 8, 2352-2363 (1973).

2. M. S. Moore, L. C. Leal, G. De Saussure, R. B. Perez, and N. M. Larson, Nucl. Phys. A 502, C443 (1989).

3. D. C. Larson, J. K. Dickens, R. O. Nelson, and S. A. Wender, "White Source Gamma-ray Production Spectral Measurement Facilities in the U.S.A.," in Proc. Int. Conf. Nuclear Data for Science and Technology, ed. S. M. Qaim, Springer Verlag (1981) 422.

4. S. A. Wender, S. Balestrini, A. Brown, R. C. Haight, C. M. Laymon, T. M. Lee, P. W. Lisowski, W. McCorkle, R. O. Nelson, W. Parker, and N. W. Hill, Nucl. Inst. and Meth. in Phys. Res. A 336, 226 (1993).

5. A. Michaudon and S. A. Wender, Performance of the LANSCE-WNR Facility Pulsed Neutron Source for Neutron Nuclear Physics, Los Alamos National Laboratory report LA-UR-90-4355 (1990).

6. P. E. Koehler, Nucl. Instr. Meth. in Physics Research A460, 352 (2001).

7. P. W. Lisowski, C. D. Bowman, G. J. Russell, and S. A. Wender, Nucl. Sci. Eng. 106, 208 (1990).

8. J. L Ullmann, private communication (2005).

9. B. E. Takala, private communication (2005).

10. WNR web page: http://wnr.lanl.gov

11. T. Granier, L. Pangault, T. Ethvignot, R. C. Haight, X. Ledoux, V. Meot, Y. Patin, P. Pras, M. Szmigiel, R. S. Rundberg, and J. B. Wilhelmy, "Measuring Neutron-Induced Fission Cross Section of Short-lived Actinides Using a Lead Neutron-Slowing-Down Spectrometer," Nucl. Instr. Meth. in Phys. Res. A506, 149 (2003).

12. D. Rochman, R. C. Haight, J. M. O'Donnell, A. Michaudon, S. A. Wender, D. J. Vieira, E. M. Bond, T. A. Bredeweg, A. Kronenberg, J. B. Wilhelmy, T. Ethvignot, T. Granier, M. Petit, and Y. Danon, "Characteristics of a Lead Slowing-Down Spectrometer coupled to the LANSCE Accelerator," Nucl. Instr. Meth. in Phys. Res. (in press).

13. L. A. Bernstein, J. A. Becker, P. E. Garrett, W. Younes, D. P. McNabb, D. E. Archer, C. A. McGrath, H. Chen, W. E. Ormand, M. A. Stoyer, R. O. Nelson, M. B. Chadwick, G. D. Johns, W. S. Wilburn, M. Devlin, D. M. Drake, and P. G. Young, Phys. Rev. C 65, 021601 (2002).

14. D. Rochman, R. C. Haight, J. M. O'Donnell, and M. Devlin, "Neutron-Induced Studies at FIGARO Using a Spallation Neutron Source," submitted to Nucl. Instr. Meth. in Phys. Res. A523, 102 (2004).

15. T. Ethvignot, M. Devlin, R. Drosg, T. Granier, R. C. Haight, B. Morillion, R. O. Nelson, J. M. O'Donnell, and D. Rochman, "Prompt Fission Neutron Average Energy for ${ }^{238} \mathrm{U}(\mathrm{n}, \mathrm{f})$ from Threshold to $200 \mathrm{MeV}$,” Physics Letters B575 (2003), 221.

16. F. B. Bateman, R. C. Haight, S. M. Sterbenz, M. B. Chadwick, P. G. Young, and S. M. Grimes, "Light Charged-particle Production from Neutron Bombardment of Silicon: Role of Level Densities and Isospin,” Phys. Rev. C 60, 064609-1 (1999).

17. M. Heil, R. Reifarth, M. M. Fowler, R. C. Haight, F. Käppeler, R. S. Rundberg, E. H. Seabury, J. L. Ullmann, and K. Wisshak, "A $4 \pi \mathrm{BaF}_{2}$ Detector for $(\mathrm{n}, \gamma)$ Cross Section Measurements at a Spallation Neutron Source,” Nucl. Instr. Meth. in Phys. Research A459, 229-246 (2001).

18. R. Reifarth, T. A. Bredeweg, A. Alpizar-Vicente, J. C. Browne, E.-I. Esch, U. Greife, R. C. Haight, R. Hatarik, A. Kronenberg, J. M. O'Donnell, R. S. Rundberg, J. L. Ullmann, D. J. Vieira, J. B. Wilhelmy, and J. M. Wouters, "Background Identification and Suppression for the Measurement of (n,gamma) Reactions with the DANCE Array at LANSCE,” Nucl. Instr. Meth. in Phys. Res. A531, 530-543 (2004).

19. F. Vivès, F.-J. Hambsch, H. Bax, and S. Oberstedt, Nucl. Phys. A 662, 63 (2000). 
20. R. W. Finlay, W. P. Abfalterer, G. Fink, E. Montei, T. Adami, P. W. Lisowski, G. L. Morgan, and R. C. Haight, "Neutron Total Cross Sections at Intermediate Energies," Phys. Rev. C47, 237-247 (1993).

21. W. P. Abfalterer, F. B. Bateman, F. S. Dietrich, R. W. Finlay, R. C. Haight, and G. L. Morgan, "Measurement of Neutron Total Cross sections up to $560 \mathrm{MeV}$," Phys. Rev. C 63, 044608 (2001). 


\title{
(-) Rensselaer
}

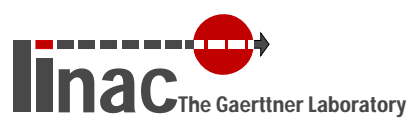

\section{The Gaerttner LINAC Laboratory Review and Current Activity}

\author{
Y. Danon, ${ }^{a,{ }^{*}}$ R. C. Block, ${ }^{a}$ J. A. Burke, ${ }^{b}$ N. J. Drindak, ${ }^{b}$ J. G. Hoole, ${ }^{b}$ \\ G. Leinweber, ${ }^{b}$ Michel Rapp, ${ }^{a}$ Frank Saglime ${ }^{a}$ \\ ${ }^{a}$ Department of Mechanical Aerospace and Nuclear Engineering, \\ Rensselaer Polytechnic Institute, Troy, NY 12180 \\ ${ }^{b}$ KAPL Inc., Lockheed Martin Corporation, Schenectady, NY 12301-1072
}

A presentation given at the ORELA workshop, Oak Ridge, TN, July 14-15, 2005

\begin{abstract}
A brief overview of the history of the Gaerttner LINAC Laboratory since its beginning of operation in 1961 is given. This includes a short description of some of the upgrades necessary for continuous operation of more than 40 years. An overview of the current and future plans of the research activity in the laboratory is also provided.
\end{abstract}

\section{INTRODUCTION AND HISTORICAL REVIEW}

The Gaerttner LINAC Laboratory started its operation in December of 1961 and is since used for research and teaching at Rensselaer Polytechnic Institute (RPI). The laboratory and LINAC were designed and built in order to perform moderator and neutron cross-section measurements in the resonance region. As such, it is a pulsed $L$ band LINAC capable of delivering up to $100 \mathrm{MeV}$ electrons in a repetition rate of up to $500 \mathrm{~Hz}$ and a pulse width that can vary from 20 ns to $5 \mu$ s with up to $10 \mathrm{KW}$ of average electron beam power [1]. In the sixties, the AEC supported the construction of the LINAC and the research, which included neutron transport in moderators, photonuclear interactions, neutron interactions and radiation effects on materials. At that time, the RPI LINAC was a new center that attracted research from RPI and outside, including groups from ORNL, KAPL, Idaho Falls, LANL, MIT, and Harwell.

In the seventies, activity related to the fast breeder program resulted in cross-section measurements in a broad energy range, from thermal to tens of $\mathrm{MeV}$ utilizing the $25 \mathrm{~m}, 100 \mathrm{~m}$ and $250 \mathrm{~m}$ flight stations. The research also included collaboration between RPI and ORNL on fissile measurements, which included for example, ${ }^{233} \mathrm{U}$ and ${ }^{239} \mathrm{Pu}$. In 1974 the AEC terminated the contract with RPI, and the ownership of the LINAC was transferred to RPI. At this point, Gaerttner, who was the LINAC laboratory director since its inception, instituted a policy of klystron preservation, which basically required running the klystrons with lower power (up to $6 \mathrm{MW}$ peak power). As a result of the policy, the lifetime of the klystrons was extended to over 25 years.

In the 80s and 90s, research funding at the Gaerttner LINAC was mostly from DOE and KAPL. This research included cross section measurements, spent fuel assay, thermal hydraulics with radiotracers, super voltage computer tomography, radiation effects on electronics, and degradation of organic pollutants. In order to support the expenses associated with maintaining and running the LINAC, irradiation services were also provided; examples were coloring of topaz gemstones and radiation effects in electronics. Spare parts were obtained from other, similar accelerators that were shut down, such as the

*Corresponding author. e-mail: danony@rpi.edu. 
ones at Yale, NBS, and Hanscom Air Force base; they provided a variety of spare parts that included klystrons.

In 1997, the RPI LINAC was cited as an ANS Historic Land Mark with the citation "This was one of the first laboratories, utilizing a high-power electron linear accelerator, that generated accurate nuclear data for the design of safe and efficient nuclear power reactors.”

Continuous operation for 36 years eventually had its toll on various components of the accelerator. In 1997, a \$1.4 million upgrade plan was executed using funding primarily from the Naval Reactors program through KAPL. This upgrade included purchase of ten new and rebuilt klystrons from Litton, improvement to the RF system, and installation of section 9, which was previously removed in order to allow a low-energy beam port. This upgrade was completed in 2000 and resulted in a $30 \%$ increase in electron beam power and neutron production. More importantly, this upgrade provided a significant extension of the lifetime of the accelerator.

Between 2002 and 2005, other upgrades were made to the accelerator. A new injection system upgrade that included parts from ANL was initiated using funding from the Naval Reactors program through KAPL. The purpose of this upgrade is to provide replacement for the injection system and to reduce the electron pulse width to enable high-energy neutron cross-section measurements. In addition to the injector, several new pulse transformers were replaced and new spare thyratrons were acquired.

This periodic upgrade of the machine over time allowed for continuous operation with minimum down time and has enabled uninterrupted activity of the research program. Overall the program resulted in more than 260 publications and helped graduate more than 150 MS and PhD students. Currently RPI is one of a few universities that can train students in measurement and analysis of nuclear data.

\section{CURRENT RESEARCH}

Current research at the Gaerttner LINAC Laboratory is mostly concentrated in the area of cross-section measurements. Other research directions include production of parametric $\mathrm{X}$ rays and production of medical isotopes. Most of the neutron cross-section data are obtained using the time-of-flight (TOF) method. For this the laboratory is equipped with several flight paths at distances of 15, 25, 100, and $250 \mathrm{~m}$ from the neutron source. Also available are several neutron-producing targets, each optimized for a different neutron energy range covering the range from $0.001 \mathrm{eV}$ to several $\mathrm{MeV}$.

The cross-section program is in collaboration with researchers from KAPL. For many years the program was concentrated on providing accurate cross-section and resonance parameters covering the energy range from $1 \mathrm{meV}$ to $2 \mathrm{keV}$, and thus vast experience has been accumulated and preserved over the years.

\section{Total and Capture Cross-Section Measurements}

The RPI-KAPL collaboration developed capabilities for "production" type measurements of total and capture cross section with a goal of providing high-accuracy resonance parameters. For this purpose two different neutron targets covering the "low" $(0.001 \mathrm{vV}-20 \mathrm{eV})$ and "high" (5-2000 eV) energy range are used. For each energy range transmission measurements are made at 15 and $25 \mathrm{~m}$, respectively, and also capture measurements at a flight path distance of $25 \mathrm{~m}$. The capture measurements are done using a $4 \pi$, 16-segment multiplicity detector, and the transmission data are collected using Li glass detectors of different thicknesses. The collected data typically consists of several sample thicknesses that are measured in the two different energy ranges. After the data are reduced to transmission and capture yield they are analyzed using the SAMMY code in order to obtain resonance parameters. Since such analysis is 
very sensitive to the resolution function, extensive work was done in order to characterize this resolution function for the different experimental configurations. Recent measurements were done on $\mathrm{Zr}, \mathrm{Sm}$, Hf, $\mathrm{Nd}$, Gd, and Nb; some measurements included enriched and diluted liquid samples.

An example of such recent data for Gd is shown in Figure 1; this plot shows several resonances that are not part of the current ENDF/B-VI evaluation.

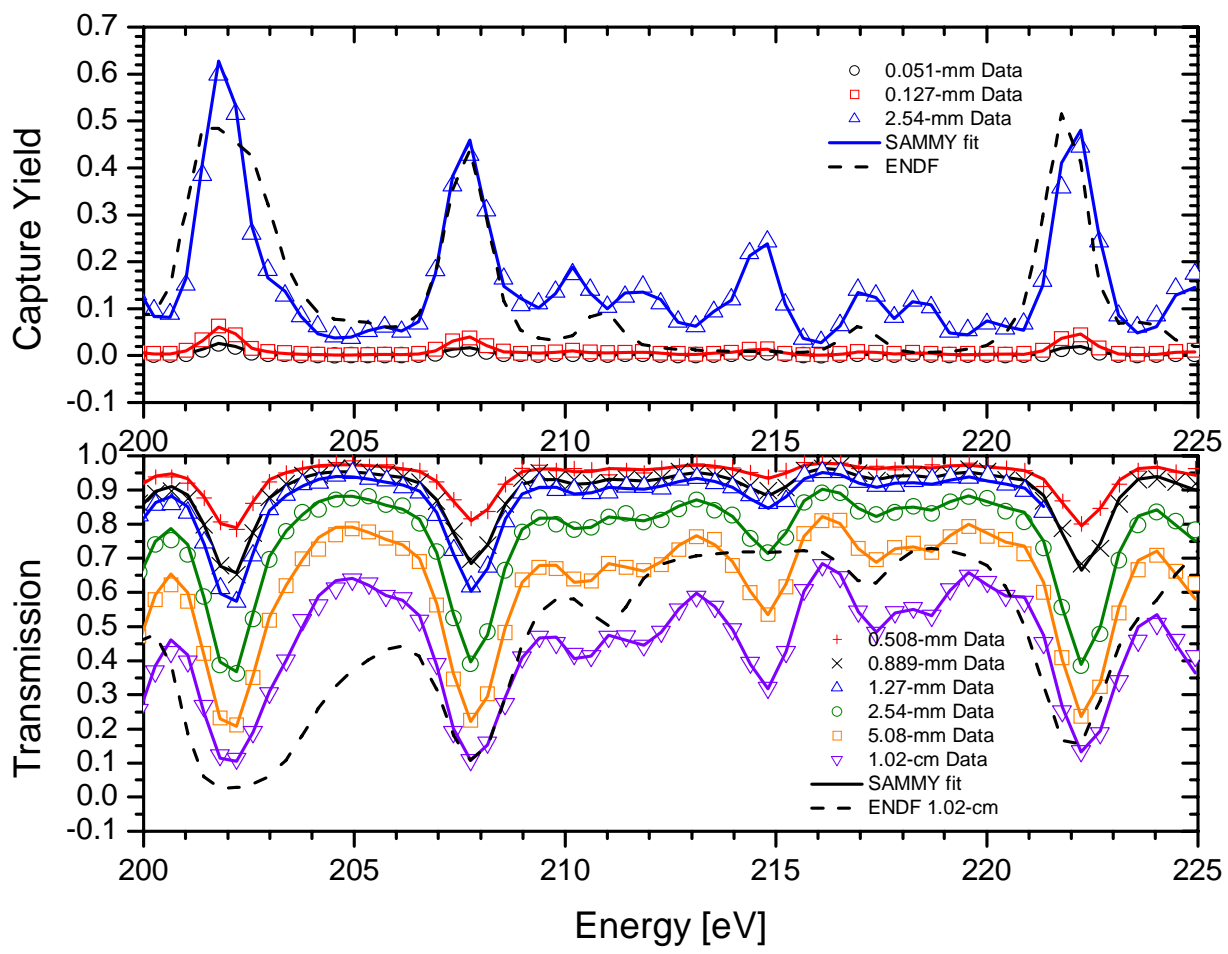

Figure 1. An example of the resonance region of natural Gd showing data and SAMMY fits for several sample thicknesses; also shown are data generated from ENDF/B-VI.

\section{New Capabilities Under Development}

New capabilities currently under development include a new transmission and quasi-integral (QI) scattering detector at the $100 \mathrm{~m}$ station, a new scattering detector array to be located at a $30 \mathrm{~m}$ flight path distance, and a new method for accurate capture-to-fission ratio measurements using the multiplicity detector.

\section{Detection System at the $100 \mathrm{~m}$ Station}

The new detector at the $100 \mathrm{~m}$ station is designed to enable transmission and spectra measurements in the neutron energy range from 0.2 to $10 \mathrm{MeV}$. The detector consists of six modular units each $7 \times 13.8$ by 5 in. thick and filled with EJ-301 (NE-213 equivalent). This type of scintillator is fast and also has capabilities to discriminate gammas by pulse shape discrimination. This detector will be used for transmission measurements and also for QI measurements. QI measurement are done by placing a sample in proximity to the neutron-producing target and measuring the scattering to a detector that is located $100 \mathrm{~m}$ away. This measurement provides a benchmark to compare with other scattering cross sections. 


\section{Scattering Detection System}

A new scattering detection array is under construction with the aim to perform differential scattering measurements in the energy range of 0.3 to $10 \mathrm{MeV}$. The system includes eight liquid scintillators ( 5 by 3 in. thick EJ-301) and a digital data acquisition system based on the Acquiris AP-240 board with DSP capabilities. The digital electronics were chosen in order to enable fast pulse shape discrimination with minimal dead time. This is necessary in order to discriminate against gamma rays produced in the scatterer. The system will be located at a flight path distance of approximately $30 \mathrm{~m}$.

\section{Measurements of Capture to Fission Ratios}

A unique method to measure capture-to-fission ratios is currently being investigated. The objective is to improve the accuracy for ${ }^{235} \mathrm{U}$ measurements. The method is based on using gamma rays from the fission and capture processes to measure the cross sections in the resonance region. Because the measured quantity is a gamma ray, such measurement allows the use of relatively thick samples. Initial experiments were done by looking at fission gammas above the binding energy of ${ }^{235} \mathrm{U}$ and also using the multiplicity information to isolate the capture gammas. Initial results are shown in Figure 2. More experiments are required in order to ensure that the detection efficiency as a function of energy and in the resonances is treated properly.

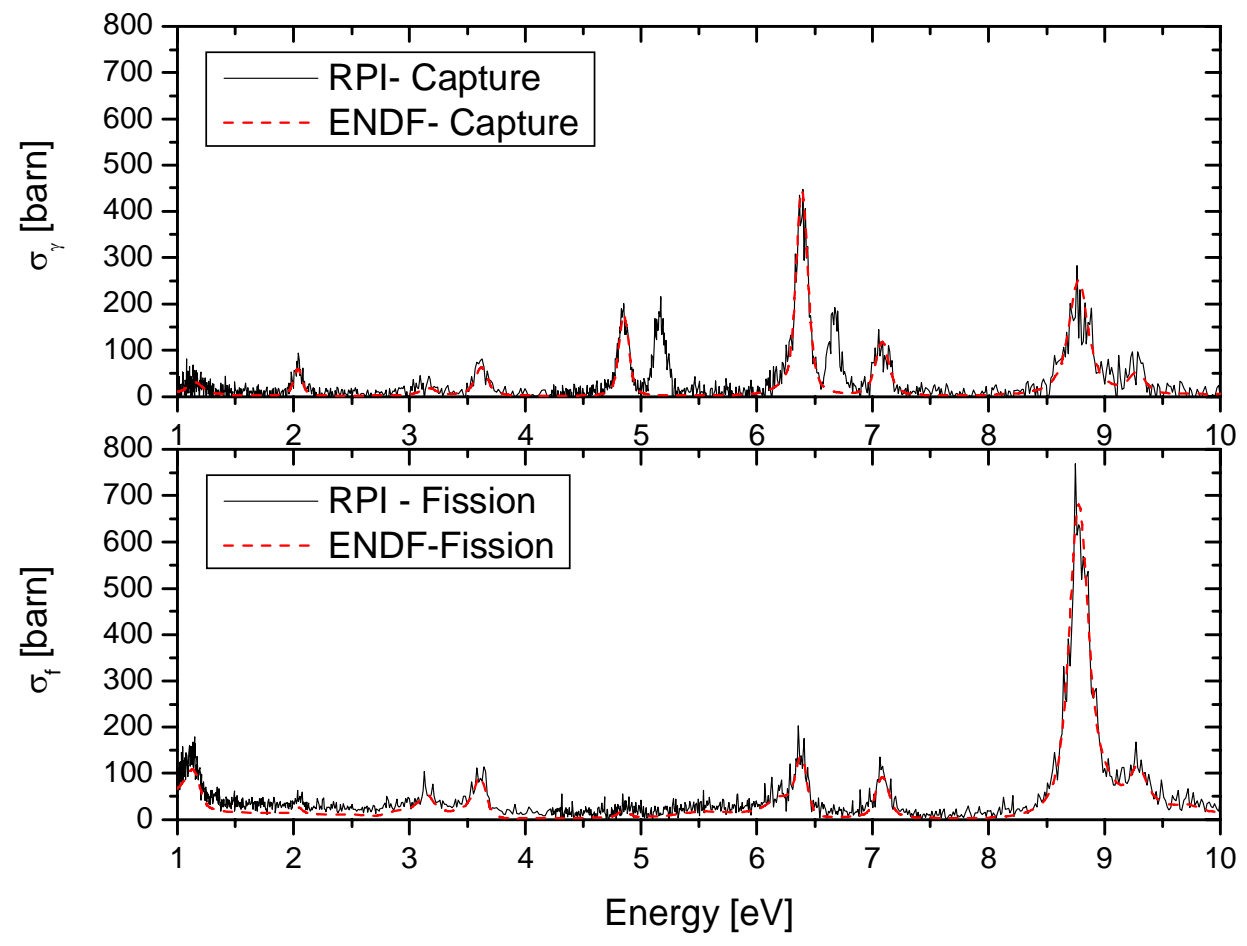

Figure 2. An example of capture and fission measurements preformed using the RPI multiplicity detector by using gamma rays emitted from capture and fission events. Two resonances in the capture measurements at $5.2 \mathrm{eV}$ and $6.7 \mathrm{eV}$ indicate contamination of ${ }^{238} \mathrm{U}$ and ${ }^{234} \mathrm{U}$, respectively, in the $93 \%$ enriched ${ }^{235} \mathrm{U}$ sample used for this experiment. 


\section{Fission Physics Measurements}

A grant from the NNSA allows us to work on development of a method to measure fission fragment mass and energy distributions as a function of the incident neutron energy and for very small sample mass. This is done by using a double-gridded fission chamber inside the RPI lead-slowing-down spectrometer. The detector and data acquisition system were already developed and are now being tested.

\section{Parametric X-Ray (PXR) Generation}

This research is not related to our nuclear data program but is aimed at using the electron beam to create a tunable, monoenergetic, directional and intense X-ray source. This can be done by using a phenomenon known as parametric X rays. In order to produce PXR the electron beam is passed through thin crystals. The interaction of the electrons with the periodic structure of the crystal produces virtual photons that are diffracted in a Bragg angle. A typical X-ray spectrum showing the energy tunability of this type of X-ray source is shown in Figure 3. One of the goals of our work is to test the validity of the theoretical models and the ability to produce high-intensity X-ray beams utilizing high electron beam currents. Using LiF

crystals and electron beam currents of up to $8 \mu \mathrm{A}$, we were able to demonstrate imaging capabilities of this type of X-ray source. Further development of a PXR source can result in a new type of intense X-ray source with applications in medical imaging and materials.

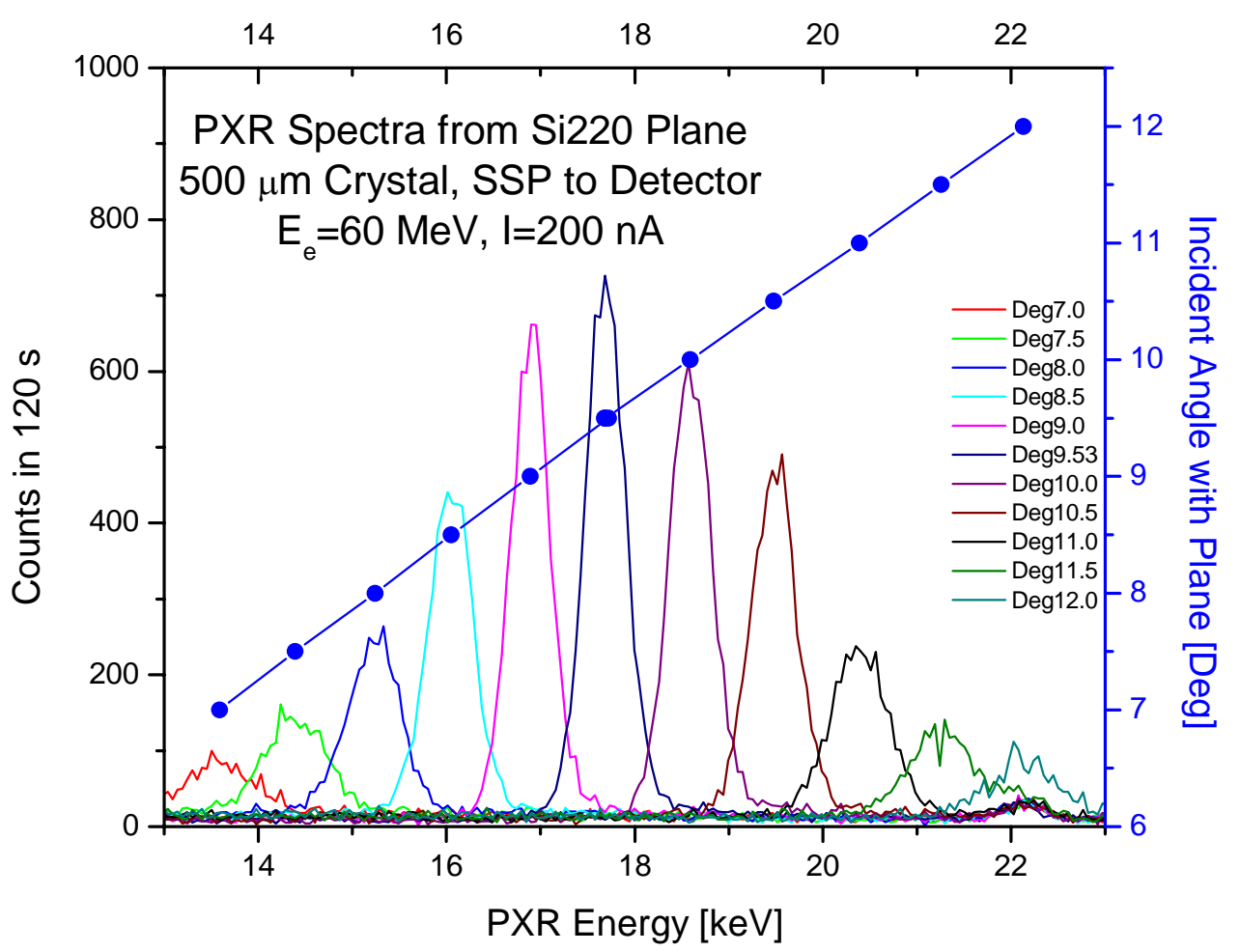

Figure 3. Several PXR spectra, each at a different energy, demonstrate the tunability feature of this type of X-ray source. 


\section{SUMMARY}

This paper presents a brief history of the Gaerttner LINAC Laboratory and the current activity. The laboratory has succeeded in maintaining continuous activity over the years since its first day of operation in 1961. This resulted from the dedication of the personnel involved in its operation and also from continuous maintenance and timely upgrades of the facility. Another key to this success is the KAPL-RPI collaboration that yielded many new cross-section measurements and also helped educate generations of engineers and physicists, of whom some are involved in the current nuclear data activity in the United States and elsewhere.

The current activity at the laboratory is still centered on cross-section measurements; however, the activity must evolve into other types of sponsored research and service in order to be able to support continuous operation of the LINAC. Such research areas and service currently include medical isotope production, novel $\mathrm{X}$-ray production, and radiation effect on electronics.

\section{REFERENCES}

1. For a complete list of refrences relevant to the work desribed here and other work using the LINAC see http://www.linac.rpi.edu/publication\%20list.pdf. 


\title{
Importance of ORELA for Developing Nuclear Reaction Models
}

\author{
F. S. Dietrich \\ Lawrence Livermore National Laboratory, Livermore, CA 94550, USA
}

\begin{abstract}
Because of its excellent energy resolution, ORELA is particularly well suited for measurements in the resolved resonance region that impact nuclear reaction model calculations. These measurements allow the determination of average level widths, level densities, and cross sections for potential scattering and radiative capture. These quantities can be used to determine parameters in reaction models (such as the optical model and Hauser-Feshbach calculations) and to understand the limitations imposed on these models. Particular attention is given to the importance of improved experimental data to characterize intermediate structure (or doorway states).
\end{abstract}

\section{INTRODUCTION}

Continuous-energy ("white") neutron sources have been used since the 1950s to measure the resonant structure in the energy dependence of neutron cross sections across the periodic table. In many applications the properties of these resonances (position, partial widths, total width) are used directly. In others, energy averages over the resonant structure are the important quantities; these energy averages determine the low-energy behavior of the nuclear optical model, which is a key ingredient in nearly all descriptions of nuclear reactions, including statistical models (e.g., Hauser-Feshbach) and distorted-wave Born approximation (DWBA) direct-interaction models. The measured average spacing of the s-wave resonances provides a critical check on the level densities used in statistical reaction models.

Since properties of individual resonances can be measured only over a limited energy range, two questions arise that affect the reliability and interpretation of the energy-averaged quantities derived from them: Have the resonances been adequately measured, with proper attention to missing resonances, separation of $s$ - and $p$-wave resonances, and separation of total spins (if the target has nonzero spin)? If so, what is the energy interval over which the energy-averaged resonance parameters applies? The first question is experimental, and recent work at ORELA (e.g., Ref. [1] for ${ }^{35,37} \mathrm{Cl}+n$ ) has shown that significant improvement on older data can be made. The second question, which is theoretical, addresses the issue of intermediate structure, which can invalidate the conventionally used forms of reaction models, such as the optical model and radiative capture. The importance of intermediate structure (frequently interpreted using the concept of doorway states [2]) can only be answered if the experimental data base is sound, and this is where ORELA can make significant contributions.

\section{FROM RESONANCES TO THE OPTICAL MODEL}

The $s$-wave strength function is defined as $S_{0}=\langle\Gamma\rangle / D$, where $\langle\Gamma\rangle$ is the energy average of the reduced neutron widths of the observed $s$-wave resonances, and $D$ is the corresponding average level spacing. This strength function can be connected to the optical model if the averaging interval is large enough to contain sufficient number of resonances but small enough that the statistical distribution of these parameters may be considered uniform within the interval. The measured values of $S_{0}$ are shown in Figure 1, together with calculations from a recent spherical global optical potential [3], shown only in mass regions where a spherical model applies. 
The peaks in the plot of $S_{0}$ are well understood to correspond to the presence of single-particle s-wave shell model states appearing close to the neutron separation energies at particular mass values (for example, $3 s_{1 / 2}$ near $\mathrm{A} \approx 60$ ). What we want to emphasize here are the deviations from the optical-model predictions, either as scatter that exceeds the experimental error bars or as a significant average deviation (as happens most prominently in the $A \approx 110-130$ region). If the experimental data are reliable, such deviations provide strong evidence that a treatment beyond the standard optical model is needed. In fact, the low values of $S_{0}$ near mass 120 have been interpreted [4] via the doorway-state model discussed in the next section.

Other useful quantities that can be determined from the resonance parameters also show similar gross structure as well as fluctuations from this average structure. These are the $p$-wave strength function $S_{1}$, and the potential scattering radius $R^{\prime}$, which characterizes the smooth $s$-wave background between resonances. These are discussed and tabulated in the compilation of Mughabghab et al. [5, 6].

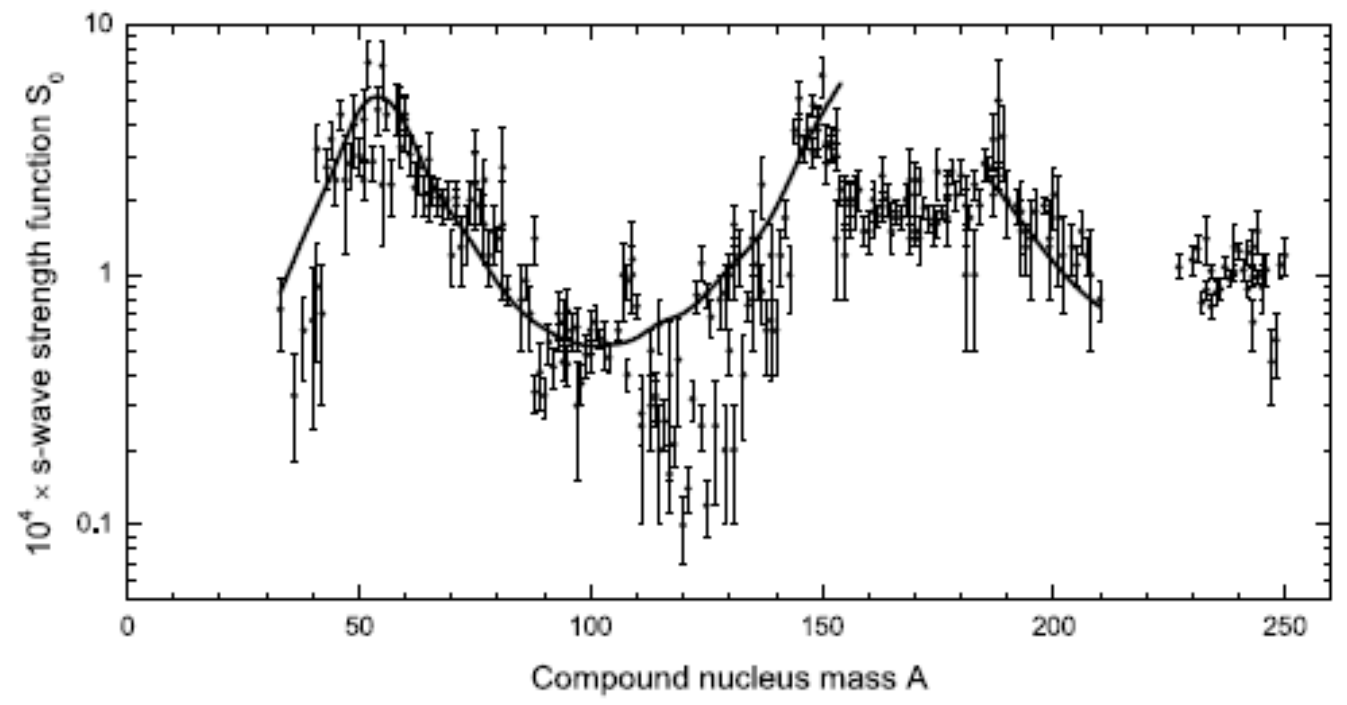

Figure 1. Experimental values of the s-wave strength function [7], compared with calculations using a global optical potential (Koning-Delaroche [3]) for spherical nuclei.

\section{INTERMEDIATE STRUCTURE AND DOORWAY STATES}

Intermediate structure refers to structure on an energy scale between that of the individual resonances seen in a high-resolution experiment and the separation between single-particle (shape) resonances such as those responsible for the gross structure seen in the s-wave strength function. An example of such structure is visible in the total neutron cross section of ${ }^{56} \mathrm{Fe}$ (see Figure 2), which shows evidence for structure with widths on the order of 100 to $200 \mathrm{keV}$.

The fluctuations in the strength functions (e.g., Figure 1) and the intermediate structure seen in Figure 2 are plausibly explained by the doorway-state hypothesis, in which the initial interaction of the neutron with the target is assumed to generate only two-particle, one-hole (2p1h) configurations of the compound system. These, in turn, damp into more complicated configurations, and still further interactions eventually reach the equilibrated compound nucleus. The theory of doorway states was developed in the 1960s (see, for example, articles by Feshbach, Kerman, and Lemmer [2] and Lane [8, 9]). If the 
doorways are well separated the strength functions (e.g., $S_{0}$ ) can be written as a sum over doorway resonances

$$
\frac{\langle\Gamma\rangle}{D} \approx \frac{1}{2 \pi} \sum_{d} \frac{\Gamma_{d}^{\downarrow} \Gamma_{d}^{\uparrow}}{\left(E-E_{d}\right)^{2}+\frac{1}{4} \Gamma_{d}^{2}} .
$$

While the reduced neutron escape width $\Gamma_{d}^{\uparrow}$ is fairly easy to estimate, the damping width of the $2 \mathrm{p} 1 \mathrm{~h}$ states into more complicated states, $\Gamma_{d}^{\downarrow}$, is poorly known. If the doorways are very dense and highly overlapping, the standard energy-averaged reaction picture (including, for example, the optical model) applies. On the other hand, if the resonances are sparse and narrow enough to retain their identity, a more complicated treatment that treats the doorways explicitly is required.

There are thus challenges to both theory and experiment: For theory, calculations need to be carried out that adequately calculate both the spectroscopic configurations of the doorways and their damping widths. For experiment, the relevant data must be measured (and old measurements revisited) to ensure that the deviations such as those indicated above from simple models are in fact real.

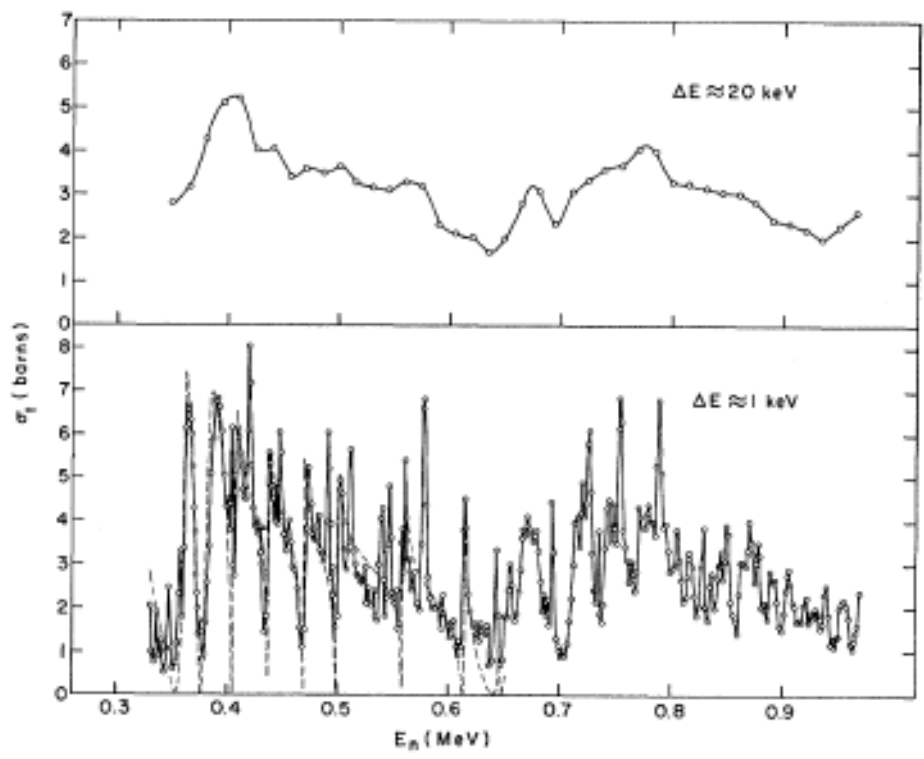

Figure 2. Total neutron cross section of natural Fe from 0.3 to $1 \mathrm{MeV}$ [10], showing evidence for nonuniform statistical behavior.

\section{RADIATIVE CAPTURE}

We discuss here two types of data on radiative capture: (1) the total capture cross section averaged over many resonances and (2) direct capture and related topics. 
Total capture cross sections are important for applications to astrophysics, reactor physics, and stockpile stewardship. In many cases they are difficult or impossible to measure directly because the targets are unstable or hard to obtain; in this case modeling calculations (Hauser-Feshbach) must be used, and their parameters must be tuned to reproduce measurements on nearby stable nuclei. As an example, the author has carried out Hauser-Feshbach calculations in the actinides that agree to about $10 \%$ with measurements on nonfissile targets; applying similar techniques to the deformed rare earth nuclei yields discrepancies at the level of 30 to 50\%, which is unsatisfactory. Improved measurements are clearly needed to separate experimental and modeling uncertainties, and ORELA has made significant progress in the last few years through rebuilding the setup for capture measurements.

Direct radiative capture, which appears as a background amplitude between resonances, needs to be understood for a number of applications to astrophysics and for data evaluation. Even though the size of the direct-capture background is so low that it is usually obscured by background effects in (n, $\gamma$ ) measurements, its integral over an energy interval containing many resonances may be large enough to constitute a significant fraction of the total average cross section. However, the theoretical techniques for estimating the direct-capture background are not fully agreed upon: the original work of Lane and Lynn $[11,12]$ used a complex optical potential in an $R$-matrix context to describe the continuum state, while Cugnon and Mahaux [13] have argued that it is satisfactory to use a real potential. Recent calculations $[14,15]$ undertaken to clarify the role of direct capture in ORELA measurements on s-d shell nuclei have used a real potential but have found significant effects from using an improved form of the electromagnetic operator plus a small contribution from semidirect capture (excitation of the giant dipole resonance). Results of these last calculations for thermal energy are shown in the first line of Table 1; they are in disagreement with other calculations by amounts up to a factor of about 2. The experimental values (last line) will exceed the direct-capture value if there is a resonance near thermal energy, as is the case for ${ }^{35} \mathrm{Cl}$. To test the various formulations of direct capture theory, it would be desirable to devise an experiment at ORELA with sufficiently low backgrounds to identify the direct capture component unambiguously. Alternatively, subtracting the resonant contribution to the average capture, which can be measured at ORELA, from the total capture (resonant plus direct) measured by an activation technique could yield the direct component if sufficient control on the systematic errors can be gotten.

Table 1. Direct-capture estimates of thermal neutron capture cross sections (in mb) for selected s-d shell nuclei, together with experimental values

\begin{tabular}{|c|c|c|c|c|c|c|c|}
\hline & ${ }^{19} \mathrm{~F}$ & ${ }^{27} \mathrm{Al}$ & ${ }^{28} \mathrm{Si}$ & ${ }^{29} \mathrm{Si}$ & ${ }^{30} \mathrm{Si}$ & ${ }^{35} \mathrm{Cl}$ & ${ }^{37} \mathrm{Cl}$ \\
\hline Present work ${ }^{*}$ & 6.5 & 60 & 151 & 111 & 98 & 430 & 418 \\
\hline TEDCA code $^{\dagger}$ & & & 65 & 58 & 67 & 160 & 310 \\
\hline Raman-Lynn $^{* *}$ & 4.6 & & 134 & 116 & 100 & & \\
\hline Lane-Lynn ${ }^{\ddagger}$ & 4.7 & 108 & 107 & 70 & 64 & & 400 \\
\hline Expt. ${ }^{\S}$ & 9.5 & 231 & 169 & 119 & 107 & 43600 & 433 \\
\hline $\begin{array}{l}{ }^{*} \text { Calculations fro } \\
{ }^{\dagger} \text { Calculations re } \\
{ }^{* *} \text { Experimental v } \\
{ }^{\ddagger} \text { External captur } \\
{ }^{\S} \text { Experimental v } \\
\text { Uncertainties } \leq 3\end{array}$ & $\begin{array}{l}{[14} \\
\text { in Re } \\
\text { rom } \\
\text { sphe } \\
\text { rom }\end{array}$ & $\begin{array}{l}9,1,20 \\
21,22 \\
\text { rmula } \\
5]\left({ }^{27} \mathrm{Al}\right.\end{array}$ & $\begin{array}{l}y \text { in } \\
\text { f. [1 } \\
\text {, [21 }\end{array}$ & ]. & 2 & & \\
\hline
\end{tabular}

Direct neutron capture proceeds only to final nuclear states that have a wave-function component that looks like the target plus a neutron. The same spectroscopy applies to a $(d, p)$ stripping reaction, and 
consequently the direct capture cross section is proportional to the spectroscopic factor measured in a $(d, p)$ reaction. This rule applies to the capture cross sections is the s-d shell in Table 1. However, in some cases this rule is completely violated, as shown in Figure 3 for ${ }^{57} \mathrm{Fe}$, in which the radiative capture and the $(d, p)$ spectroscopic factors show no correlation (or even an anticorrelation). This behavior has been interpreted using a doorway-state model; in this picture it is no accident that the total cross section of Fe shows intermediate structure (Figure 2). These results underline the importance of carefully identifying the regions of the periodic table where intermediate structure or doorway states may invalidate simple models for scattering or capture.

A related phenomenon, valence capture, predicts that there is a component of the radiative width in neutron resonances that is also proportional to the $(d, p)$ spectroscopic factor for the final state reached by gamma decay. This arises from mixing of the single-particle resonances (such as are seen in the strength functions) into the resonances. A review of this subject approximately 25 years ago [16] showed a mixed picture for the success of this model. One means of identifying the valence mechanism requires careful investigation of the correlation between neutron and gamma widths of observed resonances. Recent work at ORELA [17] on such correlations in resonances in ${ }^{88} \mathrm{Sr}(\mathrm{n}, \gamma)$ has shown that earlier experiments may have led to incorrect conclusions.

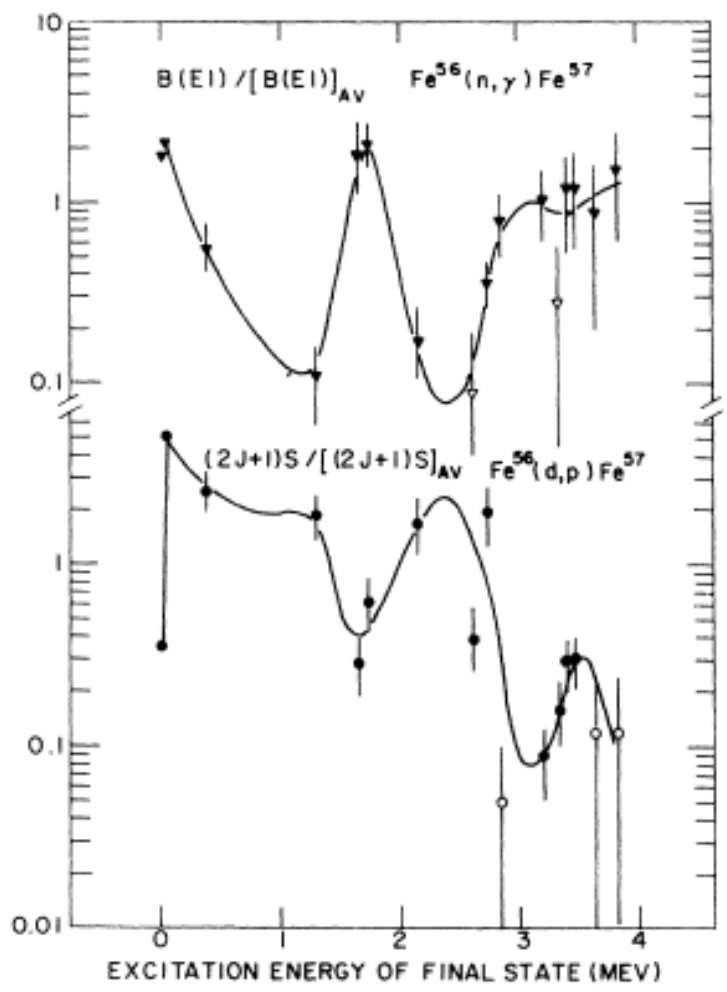

Figure 3. Comparison of $(\mathrm{n}, \gamma)$ and $(d, p)$ strengths for final states in ${ }^{57} \mathrm{Fe}[18]$. 


\section{CONCLUSIONS AND ACKNOWLEDGMENTS}

Accurate measurements of resonance parameters carried out with the high precision of which ORELA is capable can play a key role in elucidating the role of intermediate structure. There is significant evidence, some of which has been shown here, that doorway states need to be taken seriously. Lack of understanding of their properties is potentially a fundamental limitation on the validity of the optical model at low energies, and consequently a fundamental limitation on our ability to extrapolate reactionmodel calculations off the valley of stability.

Scatter in the currently available measurements of radiative capture cross sections in the unresolved resonance region suggests the presence of systematic errors that can be corrected by making measurements with the improved capture apparatus now available at ORELA.

This brief overview has focused on total cross section and capture measurements; other types of measurements that can be made at ORELA that may significantly improve reaction models include fission.

This work was performed under the auspices of the U.S. Department of Energy by the University of California, Lawrence Livermore National Laboratory, under contract No. W-7405-Eng-48.

\section{REFERENCES}

1. R. O. Sayer, K. H. Guber, L. C. Leal, N. M. Larson, and T. Rauscher, R-matrix evaluation of cl neutron cross sections up to 1.2 mev, Tech. Rep. ORNL/TM-2003/50, Oak Ridge National Laboratory, Oak Ridge, TN, March, 2003 (2003).

2. H. Feshbach, A. K. Kerman, and R. H. Lemmer, Ann. Phys. (N.Y.), 41, 230 (1967).

3. A. J. Koning and J.-P. Delaroche, Nucl. Phys., A713, 231 (2003).

4. $\quad$ C. Shakin, Ann. Phys. (N.Y.), 1, 373 (1963).

5. S. F. Mughabghab, M. Divadeenam, and N. E. Holden, Neutron Cross Sections, Vol. 1, Part A, Academic Press, New York, 1981.

6. S. F. Mughabghab, Neutron Cross Sections, Vol. 1, Part B, Academic Press, New York, 1984.

7. A. V. Ignatyuk, Average parameters of the $s$-wave resonances, available via IAEA Nuclear Data Centre web site, http://www-nds.iaea.or.at (1997).

8. $\quad$ M. Lane, Phys. Lett., 31B, 344 (1970).

9. M. Lane, Ann. Phys. (N.Y.), 63, 171 (1971).

10. E. Monahan and A. J. Elwyn, Phys. Rev. Lett., 20, 1119 (1968).

11. A. M. Lane and J. E. Lynn, Nucl. Phys., 17, 563 (1960).

12. A. M. Lane and J. E. Lynn, Nucl. Phys., 17, 586 (1960).

13. J. Cugnon and C. Mahaux, Ann. Phys. (N.Y.), 94, 128 (1975).

14. G. Arbanas, F. S. Dietrich, and A. K. Kerman, "Direct-Semidirect Neutron Capture Calculations Applied to R-Matrix Data Evaluations in the Resolved Resonance Region," in International Conference on Nuclear Data for Science and Technology, Santa Fe, NM, American Institute of Physics Conference Proceedings No. 769, 2005, p. 296.

15. G. Arbanas, F. S. Dietrich, and A. K. Kerman, manuscript in preparation (2005).

16. B. J. Allen and A. R. Musgrove, Advances in Nuclear Physics, 10, 129 (1978).

17. P. E. Koehler, R. R. Winters, K. H. Guber, T. Rauscher, J. A. Harvey, S. Raman, R. R. Spencer, J. C. Blackmon, D. C. Larson, D. W. Bardayan, and T. A. Lewis, Phys. Rev. C, 62, 055803 (2000).

18. H. Ikegami and G. T. Emery, Phys. Rev. Lett., 13, 26 (1964). 
19. H. Derrien, L. C. Leal, K. H. Guber, T. Valentine, N. M. Larson, and T. Rauscher, Evaluation of silicon neutron resonance parameters in the energy range thermal to $1800 \mathrm{kev}$, Tech. Rep. ORNL/TM-2001/271, Oak Ridge National Laboratory, Oak Ridge, TN, August, 2002 (2002).

20. K. H. Guber, R. O. Sayer, T. E. Valentine, L. C. Leal, R. R. Spencer, J. A. Harvey, P. E. Koehler, and T. Rauscher, Phys. Rev. C, 65, 058801 (2002).

21. S. Raman, E. K. Warburton, J. W. Starner, E. T. Jurney, J. E. Lynn, P. Tikkanen, and J. Keinonen, Phys. Rev. C, 53, 616 (1996).

22. S. Raman, E. T. Jurney, J. W. Starner, and J. E. Lynn, Phys. Rev. C, 46, 972 (1992).

23. S. Raman, R. F. Carlton, J. C. Wells, E. T. Jurney, and J. E. Lynn, Phys. Rev. C, 32, 18 (1985). 


\title{
Nuclear Cross Sections for Stockpile Stewardship Applications at Livermore
}

\author{
M. G. Mustafa \\ AX Division \\ Lawrence Livermore National Laboratory \\ P.O. Box 808, Livermore, CA 94550
}

August 29, 2005

\begin{abstract}
This document summarizes the presentation that I gave at the ORELA Workshop, Oak Ridge National Laboratory, on July 14, 2005. The document describes nuclear data and cross-section needs for the Stockpile Stewardship Program at the Lawrence Livermore National Laboratory. I close with some general comments about the workshop and panel discussions.
\end{abstract}

\section{INTRODUCTION}

Nuclear data and cross-section needs for the Stockpile Stewardship applications at Livermore are quite broad and include a variety of reactions and targets. Of particular importance are the energy-producing reactions and reactions for radiochemical detectors. In addition we presently need nuclear data for Homeland Security applications. Our central task is uncertainty reduction and quantification. We need very accurate $(2-3 \%)$ cross sections for the major energy-producing reactions, such as $(\mathrm{n}, \mathrm{f})$ in actinides (uranium and plutonium), to meet stockpile requirements. The other cross sections such as the (n, $\gamma$ ) and $(\mathrm{n}, \mathrm{xn})$ reactions are quite important for diagnostics. In radiochemical diagnostics we use about 23 neutron and charged particle detectors. To fully diagnose the performance of a stockpile design we need very accurate cross-section data for a network of reactions, which could include more than 100 reactions for a given detector. These reactions include both stable and unstable targets and isomeric states. We rely both on accurate measurements and on modeling for the unstable targets for which measurements are either limited by their short lifetimes or impossible with present measurement techniques. With present accuracy of cross sections we can interpret radiochemical data to better than $10 \%$ for neutron detectors and $20 \%$ for charged-particle detectors. In this report I summarize the presentation [1] that I gave at the ORELA Workshop about our needs, and point out where a facility like Oak Ridge Electron Linear Accelerator (ORELA) might be used to make new measurements or to improve on the accuracy of previous measurements. I also indicate where ORELA measurements may help in optimizing the inputs to the cross-section modeling codes.

\section{DATA NEEDS FOR RADIOCHEMICAL DETECTORS}

Radiochemical detectors were used in underground nuclear tests (UGTs) to diagnose the design characteristics. The UGT data are now used to reevaluate the performance of a design and also to test the modern codes at the Livermore supercomputers. We have data for about 23 neutron and charged particle detectors. We need a network of reaction cross sections to meaningfully apply these UGT data for stockpile applications. I illustrate this in Figure 1 for the Sm-Eu-Gd detectors [2]. The figure shows a total of 150 individual reactions involving neutrons, protons, deuterons, and tritons. This network produces a chain of reaction products of which we usually measure ${ }^{150} \mathrm{Eu},{ }^{149} \mathrm{Eu},{ }^{148} \mathrm{Eu},{ }^{147} \mathrm{Eu}$, and ${ }^{151} \mathrm{Gd}$ for diagnostics. The first four of these reaction products are produced from successive $(n, 2 n)$ reactions starting with naturally occurring ${ }^{151} \mathrm{Eu}$, and the last is the product of the $(p, n)$ and $(d, 2 n)$ reactions also on 
${ }^{151} \mathrm{Eu}$. Besides these production reactions there are destruction reactions, notably $(\mathrm{n}, \gamma)$ reactions that we worry about. The cross sections for the $(n, \gamma)$ reactions for the Sm-Eu-Gd isotopes are quite large and very poorly known.

\section{Fig. 1. Radiochemical detectors: Sm-Eu-Gd}

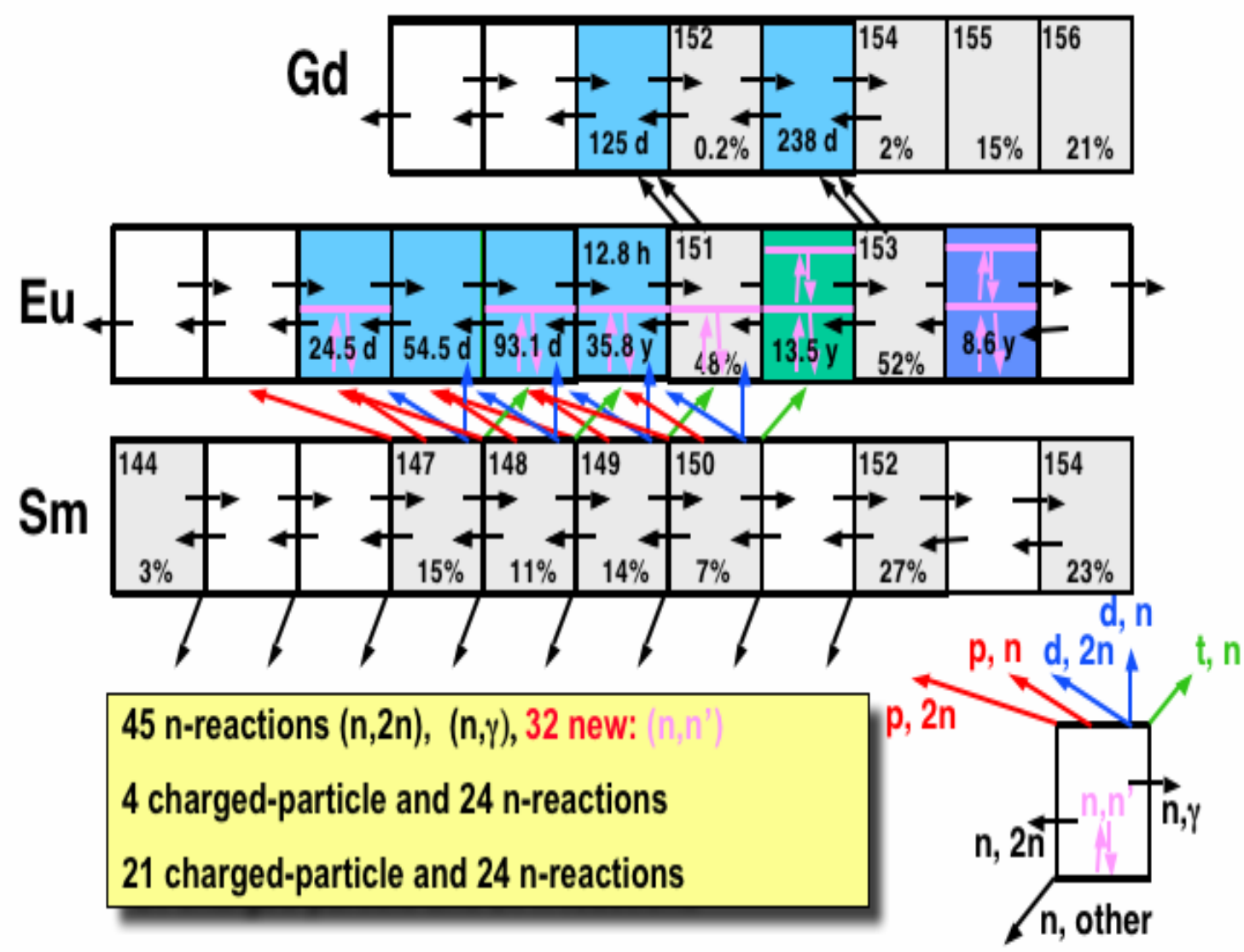

A limited number of the cross sections in this network have been measured, mostly for the stable targets. The total number of measured cross sections for the Eu and Gd isotopes is about ten. The rest of the cross sections in the network were modeled. The uncertainty estimates of both the measured and modeled cross sections are tabulated in Table 1. In general, capture cross sections have larger uncertainties, and deuteron-induced reactions are complicated from the deuteron breakup in the entrance channel. 
Table 1. Uncertainty estimates

(n,xn) reactions: $3-7 \%$ (measured); 10-15\% (modeled)

$(\mathrm{n}, \gamma)$ reactions: $10-30 \%$ (measured); 30-50\% (modeled)

(p,xn) reactions: 3-7\% (measured); 10-25\% (modeled)

(d,xn) reactions: $3-7 \%$ (measured); 15-30\% (modeled)

Measurements of capture cross sections are quite difficult and the data may have large uncertainties. We illustrate this in Figure 2 for ${ }^{151,153} \mathrm{Eu}$. Because of the large difference among various measurements, a new measurement of the ${ }^{151} \mathrm{Eu}(\mathrm{n}, \gamma){ }^{152} \mathrm{Eu}$ and ${ }^{153} \mathrm{Eu}(\mathrm{n}, \gamma){ }^{154} \mathrm{Eu}$ cross sections has been planned as a joint collaboration between Livermore and Los Alamos at the Los Alamos Neutron Science Center (LANSCE) using the Detector for Advanced Neutron Capture Experiments (DANCE) spectrometer. In addition, a capture measurement on the unstable target ${ }^{151} \mathrm{Gd}$ is also planned at Los Alamos. A suite of other capture measurement has been proposed at LANSCE for astrophysics applications. I believe ORELA can be used to complement these higher energy measurements with data for the resonance region from Oak Ridge. We at Livermore are in the early stage of getting into the capture measurements, while ORELA physicists have decades of experience in the low-energy capture measurements. Collaboration between Livermore and Oak Ridge scientists will be very valuable.

The modeling of capture cross sections has its own limitation, particularly in the keV range, where the cross sections are exponentially rising as the neutron energy is decreasing. The capture cross sections are however modeled when needed using a Hauser-Feshbach code, which requires the knowledge of optical potentials, level densities, and gamma-ray strengths. These quantities at lower energies $(\mathrm{En}<100 \mathrm{keV})$ are poorly known, and as a result the modeled $(\mathrm{n}, \gamma)$ cross sections are expected to have larger errors (see Table 1).

I mentioned a few specific cross-section measurements in which we have an immediate interest. Below is an extended list of measurements, including those that I mentioned (see Table 2). We also have need for several $(n, 2 n)$ and $(n, p)$ measurements on unstable targets, not specified in this document. 


\section{Fig. 2. Large scattering in the (n,gamma) measurements. Modeling has broad range.}

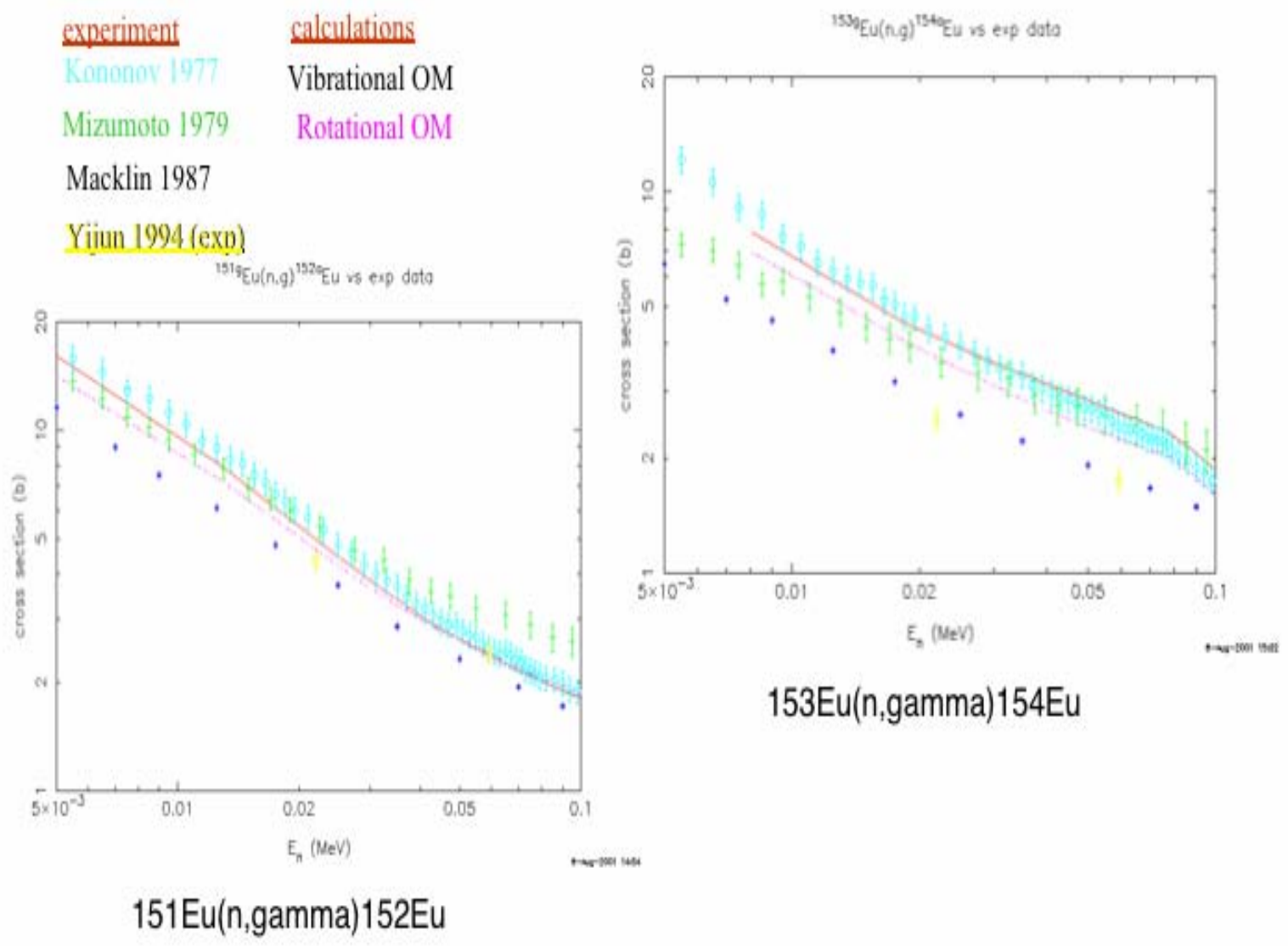

Table 2. List of (n, $\gamma$ ) measurements of interest at Livermore [3]

\begin{tabular}{lll}
\hline${ }^{151} \mathrm{Eu}(\mathrm{n}, \gamma){ }^{152} \mathrm{Eu}^{*}$ & ${ }^{153} \mathrm{Eu}(\mathrm{n}, \gamma){ }^{154} \mathrm{Eu}^{*}$ & ${ }^{155} \mathrm{Eu}(\mathrm{n}, \gamma){ }^{156} \mathrm{Eu}$ \\
${ }^{151} \mathrm{Gd}(\mathrm{n}, \gamma){ }^{152} \mathrm{Gd}^{*}$ & ${ }^{153} \mathrm{Gd}(\mathrm{n}, \gamma){ }^{154} \mathrm{Gd}$ & \\
${ }^{88} \mathrm{Y}(\mathrm{n}, \gamma){ }^{89} \mathrm{Y}$ & ${ }^{88} \mathrm{Zr}(\mathrm{n}, \gamma){ }^{89} \mathrm{Zr}$ & ${ }^{89} \mathrm{Zr}(\mathrm{n}, \gamma){ }^{90} \mathrm{Zr}$ \\
${ }^{173} \mathrm{Lu}(\mathrm{n}, \gamma){ }^{174} \mathrm{Lu}$ & ${ }^{174} \mathrm{Lu}(\mathrm{n}, \gamma){ }^{175} \mathrm{Lu}$ & ${ }^{207} \mathrm{Bi}(\mathrm{n}, \gamma){ }^{208} \mathrm{Bi}$ \\
\hline
\end{tabular}

The * indicates the reactions which are in the measurement stage. Note that, except for ${ }^{151,153} \mathrm{Eu}$, other targets are unstable. 


\section{ACTINIDE CROSS SECTIONS}

Many groups had measured the cross sections for the major energy-producing reactions, such as fission of ${ }^{239} \mathrm{Pu}$, ${ }^{235} \mathrm{U}$, and ${ }^{238} \mathrm{U}$. However, there are still major differences among various measurements and evaluations. We need to have fission and capture cross-sections measured to within an accuracy of 2 to $3 \%$ in order to meet the requirements of stockpile stewardship. (I realize that achieving 2 to 3\% accuracy may be impossible for $(\mathrm{n}, \gamma)$ reactions with present measurement techniques.) The new evaluation of the cross sections for the uranium isotopes by the ORELA group and Los Alamos is a major step in reducing some of the uncertainties. (I understand that this evaluation will be released by the end of this year as ENDFB/VII.) A similar task for the plutonium isotopes should be undertaken. The plutonium cross sections are equally important as the uranium cross sections. In addition, cross sections for americium and curium targets are useful for diagnostics. I give in Table 3 a list of capture measurements of interest for actinides.

Table 3. List of capture measurements for actinides [3]

\begin{tabular}{|c|c|c|}
\hline${ }^{238} \mathrm{Pu}(\mathrm{n}, \gamma){ }^{239} \mathrm{Pu}$ & ${ }^{239} \mathrm{Pu}(\mathrm{n}, \gamma){ }^{240} \mathrm{Pu}$ & \\
\hline${ }^{240} \mathrm{Am}(\mathrm{n}, \gamma){ }^{241} \mathrm{Am}$ & ${ }^{241} \mathrm{Am}(\mathrm{n}, \gamma){ }^{242} \mathrm{Am}$ & ${ }^{242 \mathrm{~m}} \mathrm{Am}(\mathrm{n}, \gamma){ }^{243} \mathrm{Am}$ \\
\hline${ }^{242} \mathrm{Cm}(\mathrm{n}, \gamma){ }^{243} \mathrm{Cm}$ & ${ }^{244} \mathrm{Cm}(\mathrm{n}, \gamma){ }^{245} \mathrm{Cm}$ & \\
\hline${ }^{235} \mathrm{U}(\mathrm{n}, \gamma){ }^{236} \mathrm{U}$ & ${ }^{238} \mathrm{U}(\mathrm{n}, \gamma){ }^{239} \mathrm{U}$ & \\
\hline
\end{tabular}

The capture, fission, and (n,xn) cross sections for minor uranium isotopes, such as ${ }^{236} \mathrm{U}$ and ${ }^{237} \mathrm{U}$, are also important for diagnostics. The cross sections for ${ }^{237} U$ are particularly poor. Recently, ${ }^{237} U(n, f)$ was measured using a surrogate technique [4]. This method shows promise in measuring fission and (n,xn) cross sections for other uranium isotopes and for unstable targets beyond the actinides.

\section{TOTAL CROSS SECTION MEASUREMENTS}

The ORELA is well known for measurements of total cross sections. These measurements provide information about optical potentials, which in turn gives the reaction cross sections, a key ingredient in the Hauser-Feshbach modeling of cross sections. Since the cross sections for unstable targets have to be modeled by necessity in most cases, I see that the total cross section measurements will continue to play an important role. I suggested in my presentation that such measurements be carried out in the mass 50 range. Here we are interested in the ${ }^{48} \mathrm{~V}(\mathrm{n}, \mathrm{p})^{48} \mathrm{Ti}$ cross sections. This (n,p) cross section dominates the $(\mathrm{n}, \gamma)$ at lower energies and follows the total reaction cross section. We show this in Figure 3 (dot-dash line in green color) for $n+{ }^{48} \mathrm{~V}$. The cross sections shown were all modeled using the STAPRE code [5]. I should mention that there is a future plan to try a direct measurement of this (n,p) cross section using the lead slowing-down spectrometer at Los Alamos. Unfortunately, that may still be a number of years away. In the mean time the total cross section measurement at ORELA will be of great value to us. 


\section{Fig. 3. Modeled destruction reactions for ${ }^{48} \mathrm{~V}$ $\left({ }^{48} \mathrm{~V}(\mathrm{n}, \mathrm{p})^{48} \mathrm{Ti}\right.$ dominates at lower energies)}

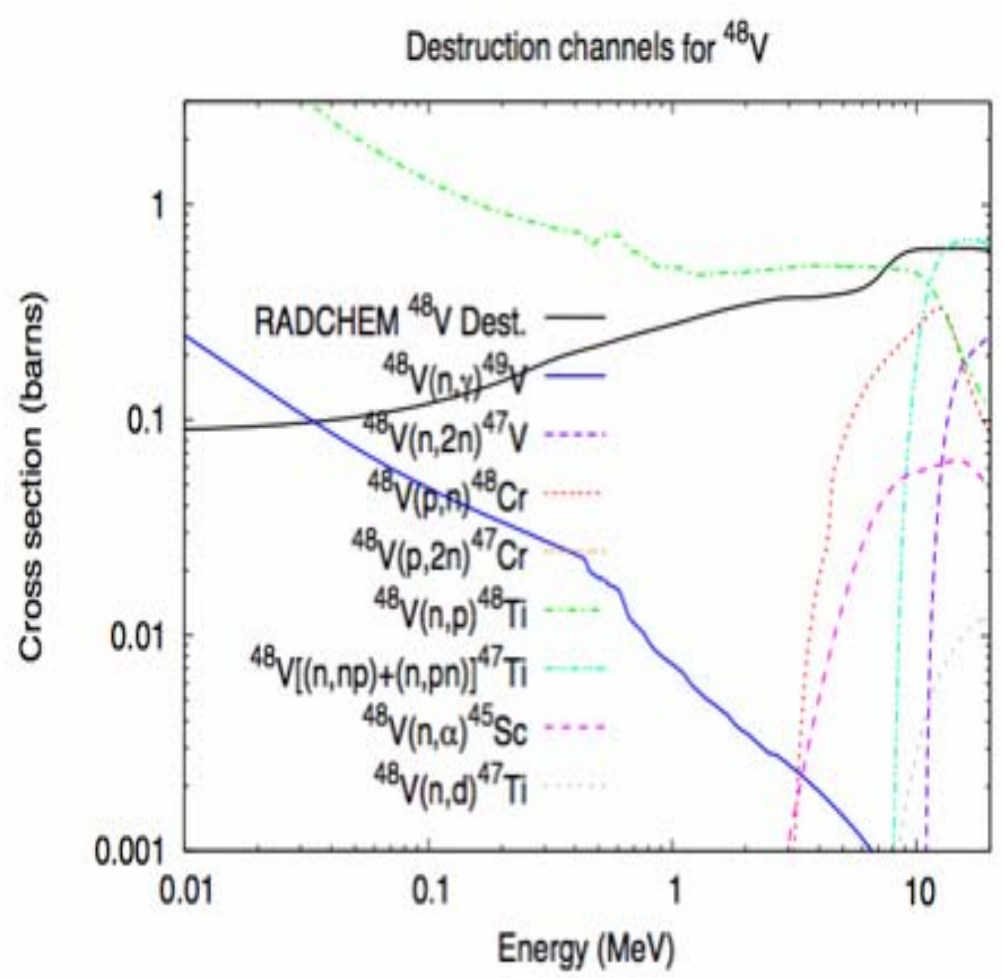

SUMMARY

I have given a partial list of measurements in which we are interested. The capture cross sections are the weakest links in the network of reactions that are needed for either the energy-producing nuclides or for radiochemical detectors. In the coming year we plan to perform a number of these measurements at the LANSCE facility at Los Alamos. The ORELA measurements in the resonance region will certainly complement the DANCE data. I would very much like to see a strong collaboration between Livermore and Oak Ridge as we have with Los Alamos.

I have a few words about the workshop and panel discussions. I learned at this workshop that there is no other facility in this country that can replace ORELA measurements in the resonance region, so I consider this a unique facility and of significant value, not only to the nuclear criticality and safety program, but also to the stockpile stewardship program as well. The resonance cross sections are not only important by themselves, but are also fundamental to the level density calculations for Hauser-Feshbach modeling of nuclear cross sections below $20 \mathrm{MeV}$. The workshop is well organized and I saw lots of enthusiasm among the participants. I think it is very important to put the necessary effort and funds to operate ORELA in a successful fashion. 
This work was performed under the auspices of the U.S. Department of Energy by University of California, Lawrence Livermore National Laboratory, under Contract W-7405-Eng-48.

\section{REFERENCES}

1. M. G. Mustafa, ORELA Workshop presentation, “Nuclear Cross Sections for Stockpile Stewardship Applications at Livermore,” Lawrence Livermore National Laboratory Report UCRL-PRES-213324, July 14, 2005.

2. R. D. Hoffman, K. Kelley, F. S. Dietrich, R. Bauer, M. Mustafa, "Neutron and Charged-Particle Induced Cross Sections for Radiochemistry in the Region of Samarium, Europium and Gadolinium," Lawrence Livermore National Laboratory Report UCRL-TR-211588, July 2005.

3. W. E. Parker, "Evaluation of Cross Section Needs (U)," Lawrence Livermore National Laboratory Report (SRD) COCA-2005-048, July 22, 2005.

4. L. Bernstein, "Surrogate measurements of ${ }^{237} \mathrm{U}(\mathrm{n}, \mathrm{f})$ cross sections," Lawrence Livermore National Laboratory, private communications, June 2005.

5. K. Kelley, R. D. Hoffman, F. S. Dietrich, R. Bauer, M. Mustafa, "Neutron and Charged-Particle Induced Cross Sections for Radiochemistry for Isotopes of Scandium, Titanium, Vanadium, Chromium, Manganese, and Iron," Lawrence Livermore National Laboratory Report UCRL-TR211668, August 2005. 


\title{
New Opportunities and Synergies for ORELA
}

\author{
G. E. Mitchell \\ Department of Physics, North Carolina State University, Raleigh, NC 27695-8202 \\ Triangle Universities Nuclear Laboratory, Durham, NC 27708-0308
}

\begin{abstract}
The measurement of neutron resonance reactions continues to be of crucial importance for both pure and applied physics. Nuclear spectra provide key tests of Random Matrix Theory and thus for chaos and complexity in nuclei. Since purity and completeness are so important, ORELA is the ideal place to perform such measurements. Neutron level densities and cross sections are critical for various aspects of stewardship science, criticality issues, and reactors. Examples are presented to demonstrate that even some of the best available data have serious limitations. The clear conclusion is that at least for key nuclides remeasurements of the neutron cross sections are needed. To maximize both the reliability and the understanding of the data, there are advantages to measuring both the neutron total cross section and the neutron capture cross section. Complementary measurements at ORELA and DANCE should provide the best possible information.
\end{abstract}

\section{INTRODUCTION}

The study of neutron resonances has been at the center of nuclear physics since the very early days. The discovery of sharp quasi-stationary states in the highly excited nuclear system led Bohr to formulate his compound nuclear hypothesis. Measurements in the resonance region and of the average cross section have been crucial for many aspects of pure and applied nuclear physics: for nuclear reactors, for criticality safety, for stockpile stewardship, for astrophysics, and for statistical theories of nuclei.

First a brief overview the history of the description of resonance reactions is presented. Although our focus for this workshop is on applications, we note the key role that neutron resonances have played in pure physics developments. To illustrate the value of neutron resonances for pure physics, we emphasize random matrix theory and the connection with chaos. To illustrate the need for high-quality data and improvements in present data sets, we discuss missing level corrections and evaluation of the relevant data quality. There are inconsistencies in a number of the existing neutron resonance data sets, most of which are now a generation old. To illustrate possible synergies, we describe some recent measurements using Detector Array for Neutron Capture Experiments (DANCE) that complement neutron measurements at ORELA. The combination of neutron capture cross sections measured at DANCE with total neutron cross sections measured at ORELA should provide the best possible information for applications.

\section{HISTORY}

In the late 1940s and throughout the 1950s, there was an explosion of measurements of neutron cross sections. Various reaction models were developed; almost all were of a statistical nature and in slightly different form remain in use today. Phenomenological level density models were developed; most followed the Bethe ansatz, and used some variation of the original Fermi gas model. Again these approaches are still very much in use today. Individual resonances and their energy, widths, and quantum numbers were characterized by the use of the Wigner-Eisenbud formalism as summarized by Lane and 
Thomas. However, the overall description of the set of resonances raised serious questions. Since for heavy nuclei the wave functions for the individual resonances have $10^{5}$ or more components, the only possible description is statistical.

Wigner proposed the use of random matrices to describe the level statistics and the width distributions. Dyson extended the theory and proposed a number of useful measures. The predicted statistical properties of the spectra are usually called Wigner-Dyson statistics. Standard measures include the Wigner surmise (the expression for the nearest-neighbor spacing distribution (NNSD) that shows the famous level repulsion), the Dyson-Mehta $\Delta_{3}$ statistic that measures the long range order, and the PorterThomas distribution for the reduced widths that is characterized by a very large number of small widths.

Although the theory was well formulated by 1963, there were no data of sufficient purity and completeness to test the theory for a long time. In 1983 Bohigas combined the best available resonance data and demonstrated that the data agreed well with the expected Gaussian Orthogonal Ensemble (GOE) version of Random Matrix Theory (RMT). About this time Bohigas also made his famous conjecture that connected the character of the level statistics (Poisson or GOE) with the degree of chaoticity (regular or chaotic). Applications of RMT have since expanded exponentially, in fields as diverse as quantum dots and lattice gauge calculations. A comprenhensive review of RMT is provided by Guhr et al. [1].

However, there has been relatively little progress in nuclear physics due to the stringent requirements on the data. The original evaluation [2] of the best resonance data is now over 20 years old. The only other large scale evaluation was of low-lying states [3] and is now nearly 15 years old. These studies should be redone and additional measurements are in order. It has been suggested that these compilations be reexamined (B. R. Mottelson [4]) and that some of the key heavy nuclei be remeasured (O. Bohigas [5]). Since ORELA is arguably the best place to perform such experiments, there are many opportunities to provide important information for the exciting area of chaos and complexity in nuclei.

\section{MISSING LEVEL CORRECTION METHODS}

\section{Standard Width Correction Method}

The Gaussian assumption for the distribution of reduced width amplitudes leads to the Porter-Thomas distribution for the dimensionless strength parameter $y$ :

$$
P(y)=\frac{1}{\sqrt{2 \pi y}} e^{-\frac{y}{2}}
$$

where $\mathbf{y} \equiv \gamma^{2} /\left\langle\gamma^{2}\right\rangle, \gamma^{2}$ is the reduced width, and $\left\langle\gamma^{2}\right\rangle$ is the average reduced width. By construction all levels weaker than the weakest observed level are missed, and one assumes that all levels with larger widths are observed.

This method works fairly well in the absence of nonstatistical effects. However, if nonstatistical effects such as doorway states are present, the result is that the average value of the reduced widths is overestimated and the cutoff parameter $y_{0}$ is therefore too small. If the nonstatistical effect is well understood, then it can be quantified and removed from the data set before analysis. However, even in favorable cases this is often difficult to achieve, and in many cases the origin of the anomaly is not understood. In order to improve the reliability of the analysis and to provide an independent test, we developed a method based on the spacing distribution. 


\section{New Spacing Correction Method}

The energies of nuclear resonances with the same quantum numbers form a GOE eigenvalue sequence. The nearest-neighbor spacings of perfect GOE sequences are to a good approximation described by the Wigner distribution [6]

$$
P_{G O E}(x)=\frac{\pi x}{2} e^{-\pi x^{2 / 4}},
$$

where $\mathrm{x} \equiv S / D, S$ is a spacing between adjacent levels and $D$ is the average spacing. We need the spacing distribution of an incomplete (imperfect) sequence. Because the positions of missing levels are random, the spacing distribution is affected by missing levels in a more complicated way than is the width distribution.

Some of the nearest-neighbor levels in the imperfect sequence are not actual nearest neighbors, due to levels missing between the observed levels. Thus the NNSD for the imperfect sequence reflects the presence of higher-order spacing distributions. The observed NNSD for an imperfect sequence can be written as

$$
P(x)=\sum_{k=0}^{\infty} a_{k} \lambda p(k ; \lambda z) .
$$

The parameter $z$ is defined as $z \equiv f x$, where $f$ is the observed fraction of levels $\left(f=N_{\text {observed }} / N_{\text {true }}\right)$. The parameters $\mathrm{a}_{k}$ give the relative contributions of the $k$-th NNSDs $p(k ; \lambda z) . \quad \lambda$ is a parameter that characterizes the incompleteness of the sequence.

The detailed derivation of the NNSD for imperfect sequences is given in [7]. The final expression is

$$
P(x)=\sum_{k=0}^{\infty} f(1-f)^{k} p(k ; x) .
$$

This result is general and therefore applies to any of the ensembles of RMT. To choose a particular ensemble requires specifying the appropriate $p(k ; x)$ for that ensemble.

Since the spacing analysis was new and thus unproven, we tested the analysis method on numerically generated data with very good statistics. We generated GOE sequences and then randomly removed a fraction $1-f$ of the levels. We applied the method to these imperfect data sets and obtained excellent agreement with the known values of missing levels. 


\section{Comparison with Data}

For data without nonstatistical effects the two methods agree fairly well. Even when the two methods agree well with each other, the result is a value in which there is greater confidence and that has a smaller uncertainty. However, the presence of nonstatistical effects has a severe impact. The additional strength added to the (true) background strength by a doorway state may significantly increase the average reduced width $\left\langle\gamma^{2}\right\rangle$, and thus incorrectly decrease the cutoff value $y_{0}$. The net result is that the number of missing levels in underestimated. Of course if one knows the nature of the nonstatistical effect, then this contribution can be evaluated and subtracted before performing the missing level analysis.

We illustrate this with data from the $\mathrm{n}+{ }^{238} \mathrm{U}$ reaction. We consider $237 \mathrm{~s}$-wave resonances identified by Olsen et al. [8, 9]. Analysis using the width correction method for the missing levels yields $f=0.97_{-0.08}^{+0.03}$. The data are considered to be essentially perfect. We then analyzed these same ${ }^{238} \mathrm{U}$ resonances with the spacing correction method. The spacing analysis method yields a value of $f=0.89 \pm 0.06$.

Are the data essentially perfect (as implied by the width correction method), or are there approximately $10 \%$ missing levels? A very strong indication of which to choose is provided by inspection of the spacing distribution. There are a number of observed spacings greater than $x=3$ and some even greater than $x=4$. However, for the Wigner distribution, the probability of $x=3$ is 0.001 , and the probability of $x=4$ is much much lower! By inspection there must be a number of missing levels.

To consider whether this specific disagreement was unique or commonplace, we considered a number of existing neutron resonance data sets that were considered of high quality [10]. For $s$-wave resonances with a sample size of order 100 or greater, the average disagreement for the most likely value of $f$ obtained by the two methods was $5 \%$, while for $s$-wave resonances with smaller sample sizes (30-60) the average disagreement between the two methods was $8 \%$. Thus for $s$-wave resonances the data appear to be of high quality. It is interesting that the correction methods appear to work (although not quite as well) even for small sample sizes. However, even for these high-quality s-wave data, there are often changes of $10 \%$ in the value of $f$, and therefore of the level density. For $p$-wave resonances the problem becomes more serious. The average difference in the $f$ values obtained by the two methods was about $15 \%$ for $1 / 2^{-}$ resonances, and the average value of $f$ is much lower. Neither of the methods work as well when a large fraction of the levels are missed. Since the data and original analysis are typically 30 years or more old, a thorough reexamination and possible remeasurement of key nuclides would seem in order.

\section{NEUTRON CAPTURE}

ORELA has excellent time-of-flight resolution. Coupled with a relatively large flux and versatility, this provides excellent opportunities for both pure and applied physics. In particular ORELA provides the perfect complement to the new DANCE array located at LANSCE. DANCE is an array of barium fluoride crystals (162 segments with 160 crystals). The very high efficiency of DANCE (this is a calorimeter that identifies capture by the $\gamma$-ray total energy) makes possible the study of very small samples, including radioactive samples. Many of the details of this system, as well as proposed applications, are given on line in the proceedings of the workshop New opportunities and challenges with DANCE [11].

The advantages of combining measurements of the neutron capture cross section at DANCE with measurements of the neutron total cross section at ORELA are many. These two facilities are arguably the best for measurements of neutron capture and neutron total cross sections. Combining the two 
measurements ensures the highest possible data quality. In particular for measurements in the resonance region, the two sets of measurements will provide the capability to determine very well both the neutron and the capture partial widths. Recent measurements on the stable europium isotopes ${ }^{151} \mathrm{Eu}$ and ${ }^{153} \mathrm{Eu}$ have provided excellent cross-section data as well as unprecedented determination of the multiplicity distributions of the statistical $\gamma$-ray cascade. Since the europium isotopes are considered so important for both stewardship science and for nuclear astrophysics, we are planning to remeasure the neutron total cross sections for these nuclides at ORELA. The combined data and analysis should provide the best possible information on the cross sections and widths. Future combined measurements are anticipated. Measurement of the gadolinium isotopes ${ }^{152,154} \mathrm{Gd}$ are planned at DANCE.

These recent measurements at DANCE, as well as proposed measurements at ORELA, provide not only valuable information for applications (their basic mission), but also provide key answers to pure physics issues. For example, the ability to measure the multiplicity distributions of the $\gamma$-ray cascades suggests a new way to learn about the characteristics of the radiative strength function at low photon energies. (The behavior of the radiative strength function affects the population of the excited states below the neutron separation energy, and thus the number of $\gamma$ rays in the cascade. Since one can predict the multiplicity distribution given the radiative strength function, one should to be able to invert the logic and determine the energy dependence of the radiative strength function from the experimental multiplicity distribution.) Increased cooperation between DANCE and ORELA should provide significant mutual benefits and valuable new information.

\section{SUMMARY AND CONCLUSIONS}

ORELA provides the capability to measure neutron total cross sections with excellent characteristics. The need for more and better measurements is well documented (see many applications covered in this workshop). In addition to the specific applications, these needs are strongly reinforced by a general overview. Most of the relevant cross sections were measured at least a generation ago. The technology and the analysis procedures have improved enormously since the earlier measurements and analyses. Recent studies suggest flaws in error correction methods used previously. Dramatically improved capabilities for performing neutron capture experiments (DANCE) suggest that there are strong mutual advantages in performing both neutron capture and total neutron cross-section measurements and combining the two data sets. These efforts should be concentrated on key nuclides that provide crucial information, for example, stewardship science.

\section{ACKNOWLEDGMENTS}

This work was supported in part by the U.S. Department of Energy under grants Nos. DE-FG02-97ER41042 and DE-FG03-03NA00076.

\section{REFERENCES}

1. T. Guhr, A. Müller-Groeling, and H. A. Weidenmüller, Phys. Rep. 299, 189 (1998).

2. $\quad$ O. Bohigas, R. U. Haq, and A. Pandey, Phys. Rev. Lett. 54, 1645 (1982).

3. J. F. Shriner, Jr., G. E. Mitchell, and T. von Egidy, Z. Phys. A338, 309 (1991).

4. B. R. Mottelson (private communication).

5. O. Bohigas (private communication).

6. E. P. Wigner, in Statistical Theories of Spectra: Fluctuations, edited by C. E. Porter (Academic Press, New York, 1965), p. 199.

7. U. Agvaanluvsan, G. E. Mitchell, J. F. Shriner, Jr., and M. P. Pato, Nucl. Instrum. Methods A498, 459 (2003). 
8. D. K. Olsen et al., Nucl. Sci. Eng. 69, 202 (1979).

9. $\quad$ D. K. Olsen, Nucl. Sci. Eng. 94, 102 (1986).

10. D. Dashdorj and G. E. Mitchell (unpublished).

11. DANCE workshop proceeding http//wnr.lanl.gov/dance/workshop2004/. 


\title{
Panel Discussion Minutes-Future Direction and Activities
}

\author{
ORELA Workshop
}

July 14-15, 2005

\author{
Moderator: $\quad$ Tim Valentine (ORNL) \\ Panelists: $\quad$ Yaron Danon (RPI) \\ Arthur Kerman (MIT) \\ Jerry McKamy (NNSA/NA-117) \\ Mohammed Mustafa (LLNL) \\ Dave Nigg (INL)
}

Identify specific applications that could benefit from ORELA's measurement capabilities?

\section{Arthur Kerman (MIT):}

- ORELA has high-resolution capabilities at low energies, which is important to see the resonance detail.

- One thing I have learned this week is the importance of ORELA measurements for input to nuclear model calculations. We need to learn more about level densities for nuclear model calculations. Gary Mitchell (TUNL) brought this out in his talk.

- ORELA is unique because of the short pulse capability for resolution.

- It would be a shame to let the facility disappear.

- The question for the community is "What would be the cost to rebuild this capability?-Hundreds of millions of dollars?”

- The cost is minimal to keep the facility running.

\section{Mohammed Mustafa (LLNL):}

- Europium isotope cross-section measurement will be performed at LANSCE, and complementary ORELA measurements would be helpful to get accurate resonance measurements.

- Resonance measurements are important for nuclear model calculations.

- We need measurements for the mass 50 range to get accurate resonance parameters.

- Total cross-section measurements are important.

- This meeting is exciting because of the excitement over resolution capability of ORELA for resonances.

- $\$ 1.5 \mathrm{M}$ is such a small item relative to the LLNL program.

- Nobody in this country can do these measurements.

Jerry McKamy (NNSA/NA-117):

- We are getting close to remeasuring beryllium (Be).

- We have developed approaches to address calculation biases, but the issue is the differential data.

- In the Introductory slide I showed about the DOE Nuclear Criticality Safety Program (NCSP), I noted that we have workarounds for the weapons program applications. The action that I have is to look at how to address these issues for the weapons program. 
- I am impressed with the collaborative support from other programs (LLNL applications noted).

- ORELA is a key component of the NCSP.

\section{Yaron Danon (RPI):}

- I cannot comment on specific applications.

- Cross sections are needed to improve accuracy for covariance data as well as crosssection data improvement.

\section{Dave Nigg (INL):}

- ORELA is certainly complementary to other U.S. capabilities.

- We need to think about how long of a lead time we need to address measurement needs. The infrastructure must be maintained to meet future needs.

- What will be the infrastructure we need as capabilities for GEN-IV applications?

\section{Tim Valentine (ORNL):}

- Bob Haight (LANL) has noted the unique ORELA measurement capability between $50 \mathrm{eV}$ and $100 \mathrm{keV}$.

What would be the consequences for losing ORELA? Where would we go to perform these measurements?

Yaron Danon (RPI):

- RPI would be a choice, but we will have less power than ORELA.

- We have similar capabilities as ORELA.

\section{Jerry McKamy (NNSA/NA-117):}

- Belgium (i.e., Institute for Reference Materials and Measurements) is the only other choice, and this presents complications.

- Maybe RPI would be the choice.

Mohammed Mustafa (LLNL):

- You will lose expertise when you lose a facility.

- I do not see how we can get resonance detail if we lose ORELA.

\section{Arthur Kerman (MIT):}

- My observation

o 20 years ago accelerators had trouble getting parts in the U.S.

o This continues to be a problem today.

o The next step would be to put the accelerators in other countries, but it would be a mistake to let this happen.

- Physics point

o The nuclear criticality safety issue is, what is the cross-section value between resonances?

o If you cannot measure the cross-section value between resonances, you cannot get the integral right-resolution is important for this. 
Question directed to Yaron Danon and Jerry McKamy: Provide comments on the importance of facility maintenance and technical staffing?

\section{Yaron Danon (RPI):}

- Our staffing at RPI

o We have an engineer, a facility supervisor, and two techniciansadditionally, we have experimentalists and students.

0 This is the minimum facility staff needed for keeping RPI operational.

o Technical staffing is an issue for ORELA.

o ORNL needs to maintain expertise and capability to run the machine.

o In the event the technical expertise is lost, it is not easy to come back tomorrow and perform work.

o Staffing applies to those who maintain the machine and those who perform measurements.

\section{Jerry McKamy (NNSA/NA-117):}

- The NCSP is updating the Five Year Plan to include \$475K/year to address the nearterm problems.

- We hope to make a dent in the improvement needs.

- Staffing comments

o The DOE Office of Science (SC) provides some manpower support.

o How do we grow the next group of people?

o Example: Who will be the next "young gun" engineer?

o One suggestion would be to establish a collaboration with local U.S. universities to learn engineering skills needed at ORELA.

o There should be a need for accelerator physics types in the United States.

\section{Tim Valentine (ORNL):}

- We need to maintain the facility to attract students to ORELA.

Mohammed Mustafa (LLNL):

- We face the same problem in the radiochemistry field.

- We have started introducing summer students to work in radiochemistry.

Jerry McKamy (NNSA/NA-117):

- It is important to identify things to bring ORELA up to speed to keep it alive and keep it functioning reliably.

- In terms of a medical analogy, ORELA is currently in ICU, but we are close to moving it out of ICU into a hospital room.

\section{Yaron Danon (RPI):}

- Education point

o One possibility would be to establish a fellowship for students to work in nuclear data.

o We could then have students work at ORELA.

\section{Arthur Kerman (MIT):}

- $\quad$ Psychology is very important when discussing ORELA.

- The words that are used now give the impression that this is an operating facility-it is not.

- We need to use words to convey reestablishing operation. 
What are the panelists' views concerning the inoperability of the facility?

Jerry McKamy (NNSA/NA-117):

- The longer that ORELA stays down, the perception is that you can get along without the facility.

Mohammed Mustafa (LLNL):

- Los Alamos Neutron Science Center (LANSCE) went through a similar phase as ORELA about 15 years ago.

- It is easier to recreate a theory program, but it is not easy to recreate an experimental capability.

- We need collaboration between LLNL and ORELA in the next couple of years.

\section{Yaron Danon (RPI):}

- It is not practical to maintain ORELA for the sake of doing this (maintain the facility).

- We need to have directed measurement programs.

- We need to find programs to do this.

Please comment on whether you see ORELA as a vital national resource.

Jerry McKamy (NNSA/NA-117):

- Yes.

Mohammed Mustafa (LLNL):

- I started here at ORNL as a student learning physics.

- If resonances are so important, I would vote yes.

\section{Arthur Kerman (MIT):}

- The language is inappropriate.

- A vital national resource requires hundreds of millions of dollars to operate and maintain.

- We must recognize that ORELA is important.

- Relative to national resources, ORELA does not need hundreds of millions of dollars to operate and maintain.

\section{Dave Nigg (INL):}

- Yes. This language pushes a lot of buttons.

- New GEN-IV reactor concepts will present needs five years down the road and beyond.

- We need to maintain ORELA to encourage people to choose nuclear data as a career.

- ORELA is a key component of U.S. measurement programs, but do not use language such as "vital national resource."

\section{Tim Valentine (ORNL):}

- This is good advice.

- ORELA is not a vital resource, but it is a vital component of U.S. measurement capabilities. 


\section{Tim Valentine (ORNL), panel discussion closing remarks}

- ORELA fills a gap in the nuclear data area in terms of resonance resolution capability at low and intermediate energies.

- ORELA is important for the NCSP to provide criticality safety capability maintenance as specified in Defense Nuclear Facilities Safety Board (DNFSB) Recommendation 97-2.

o There are new challenges in reactor design; nuclear data will play a key role:

o higher burnup.

o feedback mechanisms.

- Advanced Fuel Cycle Initiative

o Data needs will be present, but many of the needs have yet to be determined.

- Covariance Data

0 We need to have cross-section data with uncertainty information.

o Precise measurements do not mean more accuracy.

o We must understand the data.

o This requires a cross-section measurement capability such as ORELA.

- $\quad$ Standards Data

o Some of the cross-section standards are not as good as we might think.

o New measurements will be required to obtain improved standards data.

- In summary, there is a strong and compelling case for reviving ORELA. 


\section{Appendix: Presentations}

ORELA Status \& Workshop Objectives

Michael Dunn

Nuclear Data Group Leader

Nuclear Science and Technology Division, Oak Ridge National Laboratory

The Nuclear Criticality Safety Program Infrastructure Supports Criticality Safety Engineers

DOE-Wide: NNSA Perspective on ORELA Support for the NCSP.

Jerry N. McKamy

NCSP Technical Program Manager, National Nuclear Security Administration

Nuclear Data Needs Supporting Gen-IV Applications - INL Perspective and Initiatives

David W. Nigg

Manager, Reactor and Nuclear Physics

Idaho National Laboratory

The Future of Nuclear Energy and the Role of Nuclear Data

Phillip Finck

Deputy Associate Laboratory Director

Applied Science and Technology and National Security, Argonne National Laboratory

NNDC Perspective on Nuclear Reaction Databases and ORELA.

Pavel Oblozinsky

National Nuclear Data Center, Brookhaven National Laboratory

ORNL Neutron Science Overview.

Paul Koehler

Physics Division, Oak Ridge National Laboratory

Neutron Cross Section Measurements at ORELA.

K. H. Guber, ${ }^{1}$ L. C. Leal, ${ }^{1}$ J.A. Harvey, ${ }^{2}$ R. O. Sayer, ${ }^{1}$ H. Derrien, ${ }^{1}$ D. A. Wiarda, ${ }^{1}$ and P. E. Koehler ${ }^{2}$

${ }^{1}$ Nuclear Science and Technology Division, ${ }^{2}$ Physics Division; Oak Ridge National Laboratory

LANSCE and ORELA Complementary Measurement Capabilities

Robert Haight

Los Alamos National Laboratory

The Gaerttner LINAC Laboratory Review and Current Activity

Y. Danon and R. C. Block, M. Rapp, and F. Saglime

Rensselaer Polytechnic Institute, Troy, NY

and

J. A. Burke, N. J. Drindak, J. G. Hoole, and G. Leinweber

Lockheed Martin Corporation, Schenectady, NY

LANL - ORNL Nuclear Data Evaluation Collaborations for Supporting NCSP.

M. B. Chadwick, R. C. Little, and T. Kawano

Los Alamos National Laboratory 


\section{Appendix: Presentations (continued)}

Page

Low-Energy Neutron Total and Capture Cross Sections at ORELA and

Nuclear Reaction Models

Frank S. Dietrich

Physics and Applied Technologies, Lawrence Livermore National Laboratory

Nuclear Cross Sections for Stockpile Stewardship Applications at Livermore

M. G. Mustafa

AX Division, Lawrence Livermore National Laboratory

New Opportunities and Synergies for ORELA.

G. E. Mitchell

North Carolina State University and

Triangle Universities Nuclear Laboratory

Future of Nuclear Energy in the United States .

Jim Rushton and Jeff Binder

Nuclear Science and Technology Division, Oak Ridge National Laboratory

ORELA Improvement Plan

Michael Dunn

Nuclear Data Group Leader

Nuclear Science and Technology Division, Oak Ridge National Laboratory 


\section{ORELA STATUS \& WORKSHOP OBJ ECTIVES}

\section{Michael Dunn}

Nuclear Data Group Leader

Nuclear Science \& Technology Division

ORELA Workshop

July 14-15, 2005

OAK RIDGE NATIONAL LABORATORY

U. S. Department of Energy

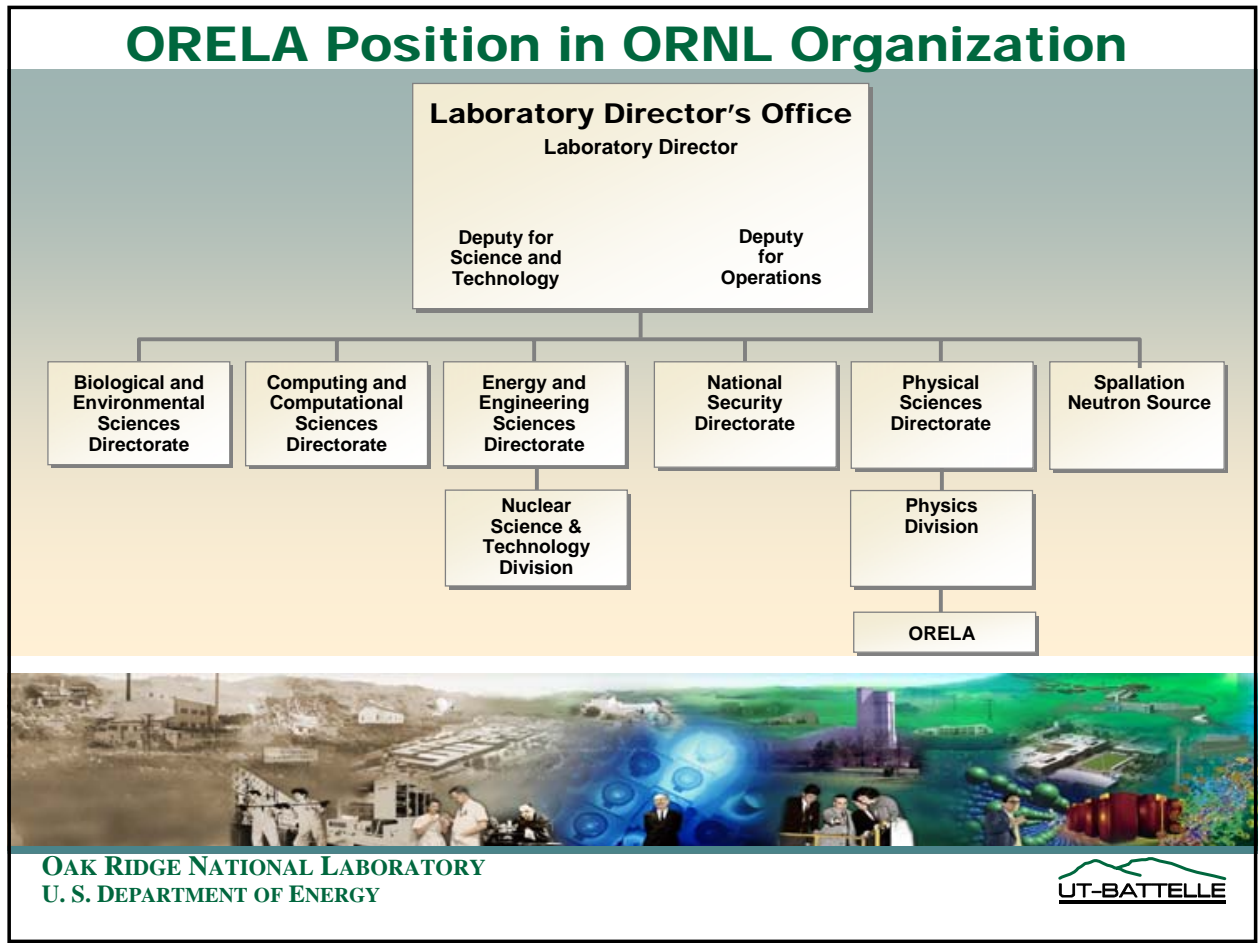




\section{What is ORELA?}

$>\underline{\text { Oak }}$ Ridge Electron Linear Accelerator

- Perform neutron cross-section measurements

- Generate electrons that strike a target (Ta) and produce neutrons via bremstrahlung and photoneutron reactions

- Neutrons directed down various flight paths to cross-section measurement stations

Important features

- Unique measurement facility in U.S.

- High flux $\left(10^{14} \mathrm{n} / \mathrm{sec}\right)=>$ gram-sized, affordable samples

- Excellent resolution $(\Delta t=4-30 \mathrm{~ns})=>$ good $\mathrm{S} / \mathrm{N}$ facilitates better evaluations

- "White" neutron spectrum from $\mathbf{E}_{\mathbf{n}}$ $0.01 \mathrm{eV}-80 \mathrm{MeV}=>$ reduces systematic uncertainties

- Measurement systems well understood => very accurate data.

- Simultaneous measurements $=>(n, \gamma)$, $(n, \alpha),\left(n, n^{\prime}\right)$, and $\sigma_{\text {total }}$ experiments at the same time on different beam lines

OAK RIDGE NATIONAL LABORATORY
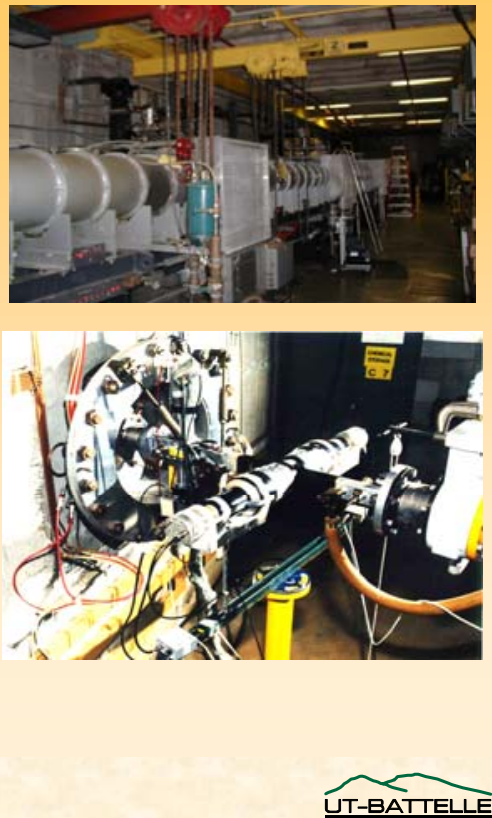

\section{ORELA is Major Contributor to DOE Science and Nuclear Missions}

ORELA provides high-resolution neutron measurements in the low to intermediate energy ranges-important for current and emerging nuclear applications

Measurement experience coupled with ORELA capabilities has established ORNL as leading evaluation center for resolved and unresolved resonance regions

> Over past 30 years, ORELA measurements have contributed to $\sim 80 \%$ of U.S. Evaluated Nuclear Data File (ENDF/B) evaluations
Energy Resolution Comparison for ${ }^{116}$ Sn Capture Measurements taken at ORELA and Karlsruhe
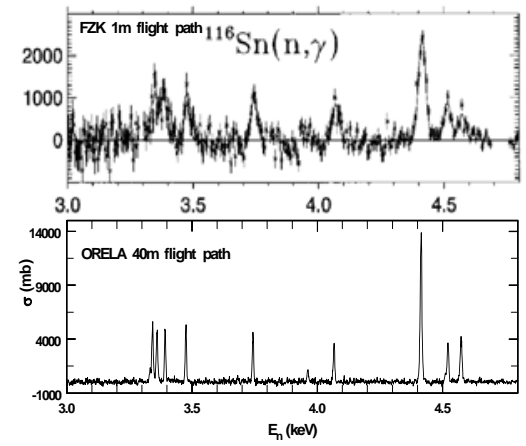


\section{ORELA: Unique World Class Machine for}

Neutron Sciences

$>$ High neutron flux $\left(10^{14} \mathrm{n} / \mathrm{sec}\right)$, detailed energy resolution, multiple beam lines, etc. make ORELA unique

$>$ ORELA complements other U.S. measurement facilities (LANSCE, SNS, HFIR etc.) by providing detailed differential cross-section data measurements in resonance region

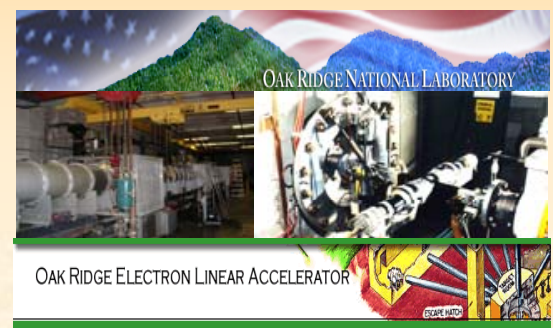

OAK RIDGE NATIONAL LABORATORY
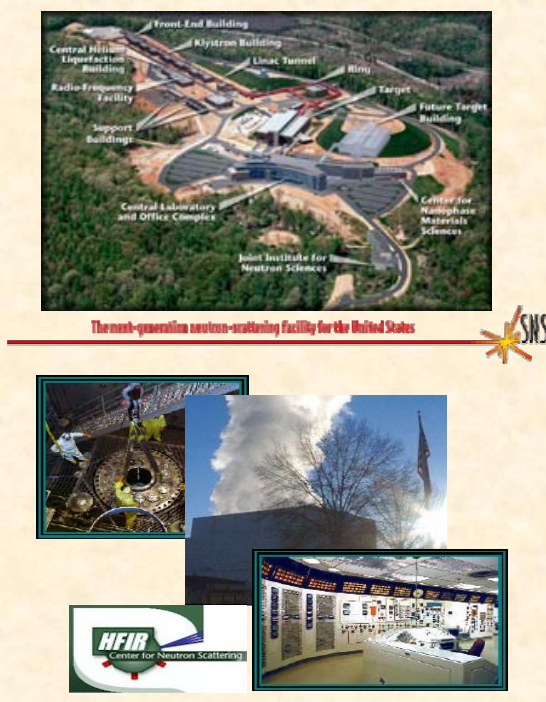

UT-BATTELLE

Complementary U.S. Cross-Section Measurement Facilities for Supporting Nuclear Applications
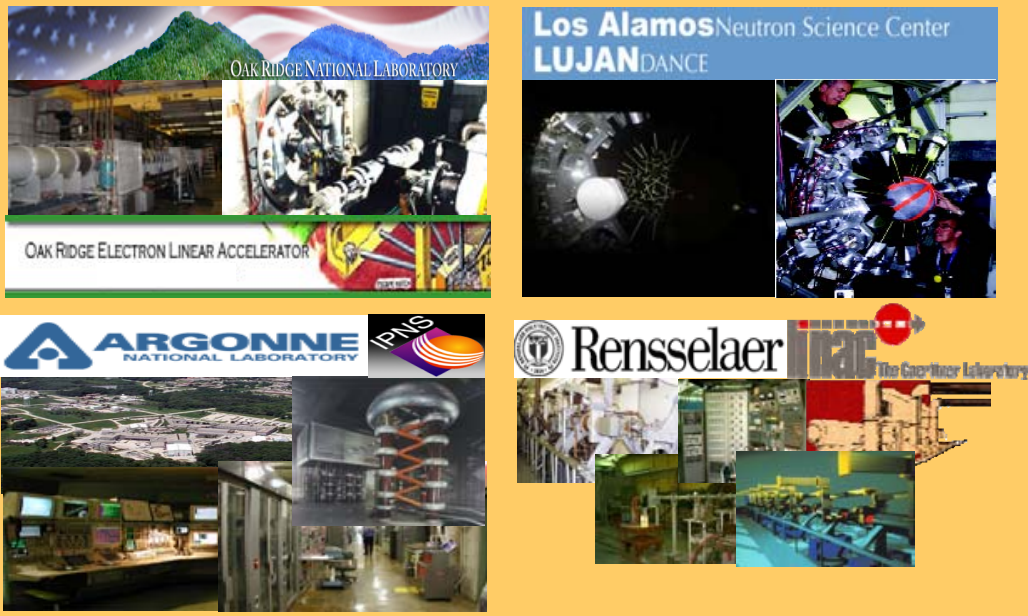

OAK RIDGE NATIONAL LABORATORY

U. S. DEPARTMENT OF ENERGY

UT-BATTELLE 

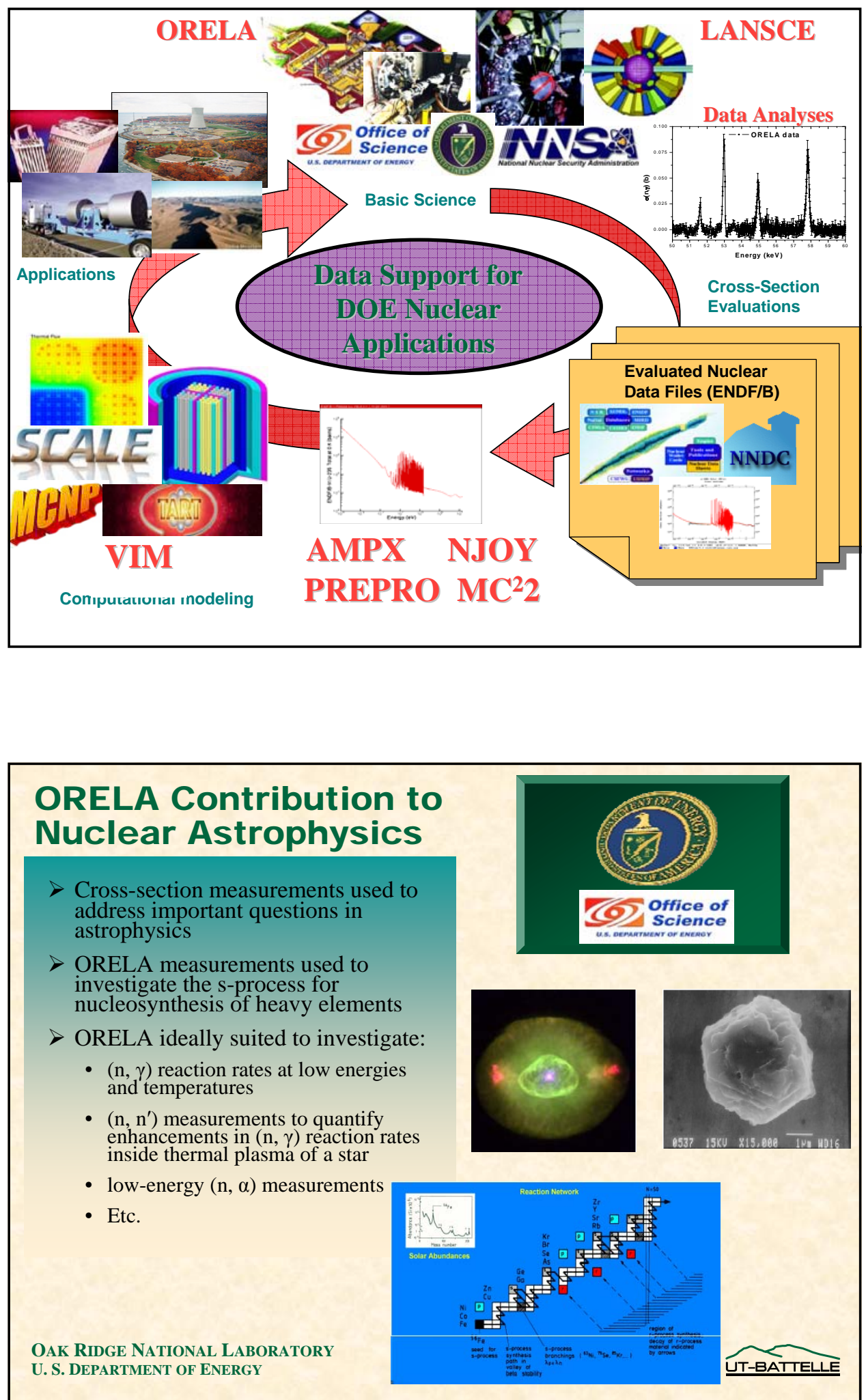

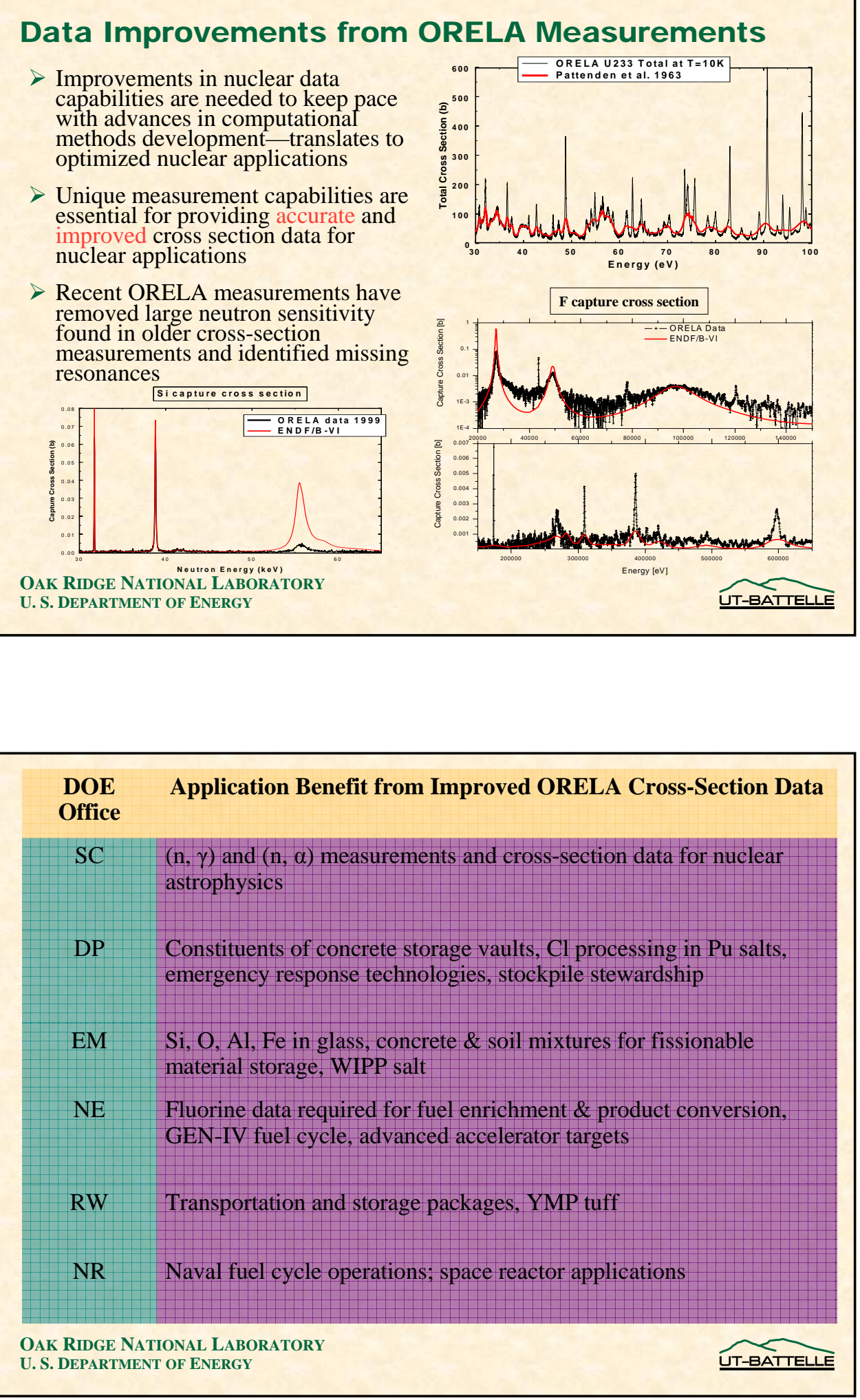


\section{ORELA Facility Status}

\section{Background (Pre 1990s)}

- In late 1960s, ORELA sponsorship split between Nuclear Energy and Nuclear Physics Programs under Atomic Energy Commission (AEC)

- ORELA sponsorship passed to DOE Office of Science (SC) with demise of Fast Reactor Program in early 1980s

\section{0s to Present}

- SC is ORELA facility owner

- ORELA provides data support for NNSA/NA-11 Nuclear Criticality Safety Program (NCSP)

\section{$>$ Current ORELA Nuclear Data Customers}

- DOE/SC and NNSA/NCSP

- DOE Office Environmental Management (EM)—Criticality Safety Applications

- DOE Office of Civilian Radioactive Waste Management (RW) - Burnup Credit (BUC) for transportation applications

- DOE Nuclear Energy (NE)--NERI graphite measurements

OAK RIDGE NATIONAL LABORATORY

\section{ORELA Facility Status}

$>$ No single sponsor provides complete base funding for facility-support leveraged with different programs

Advantages \& Disadvantages

- Sponsors benefit by receiving accurate cross-section measurements at reduced cost

- Programmatic funds are used to support program-specific goals of sponsor-ORELA does not receive base funding to support major upgrades and significant maintenance operations

Beginning in FY2002, operational problems have plagued ORELA

- Aging equipment

- Electron gun malfunctions - unique guns manufactured in house

- Inability to maintain adequate vacuum conditions in the accelerator

$>$ ORELA availability and reliability has decreased—stagnating current and new measurements

- 1 week of beam time in FY2004

- Needed 2-3 months of beam time in FY2004

OAK RIDGE NATIONAL LABORATORY

U. S. DEPARTMENT OF ENERGY

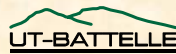




\section{ORNL Currently Addressing Facility Issues}

Facility upgrades needed to increase ORELA reliability and maintain long-term operational readiness

- \$1.5M crucial improvements

- \$1.6M desirable improvements

$>$ Improvement plan developed-more details on Friday

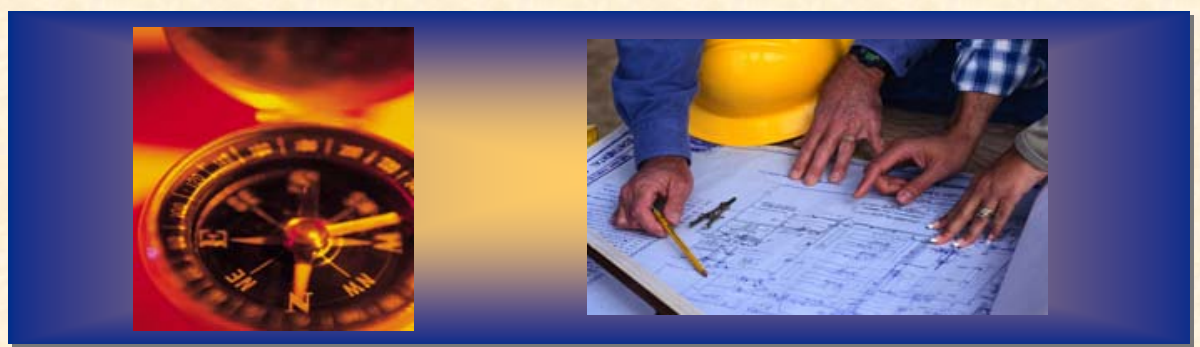

OAK RIDGE NATIONAL LABORATORY

\section{ORELA: Unique World Class Measurement Facility}

\section{Accomplishments/Capabilities Issues}

- 30+ years performing detailed energy- - Reduced availability \& resolution cross-section measurements

- Data contributions to multiple nuclear programs

- Contribution to significant number of reliability

- Misconceptions or unawareness of ORELA capabilities in technical community ENDF evaluations

- Continued need to support current and emerging nuclear applications

\section{Actions}

- Implement improvement plan to re-establish operational reliability

- ORELA Workshop key component for establishing technical community endorsement and communicating facility capabilities

OAK RIDGE NATIONAL LABORATORY

U. S. DEPARTMENT OF ENERGY 


\section{ORELA Workshop Objectives}

Clarify role of ORELA in the U. S. nuclear data measurement portfolio

$>$ Establish technical community consensus on the importance of ORELA for supporting U. S. cross-section measurement needs

\section{Mechanism}

- Series of invited talks from nuclear data and application experts from various technical backgrounds

- ORELA tour followed by panel discussion to establish conclusions from workshop

- Proceedings will be used to document the technical community consensus on ORELA 
NNSA Perspective on ORELA Support for the NCSP

\author{
Jerry N. McKamy
}

NCSP Technical Program Manager

NNSA, NA-117

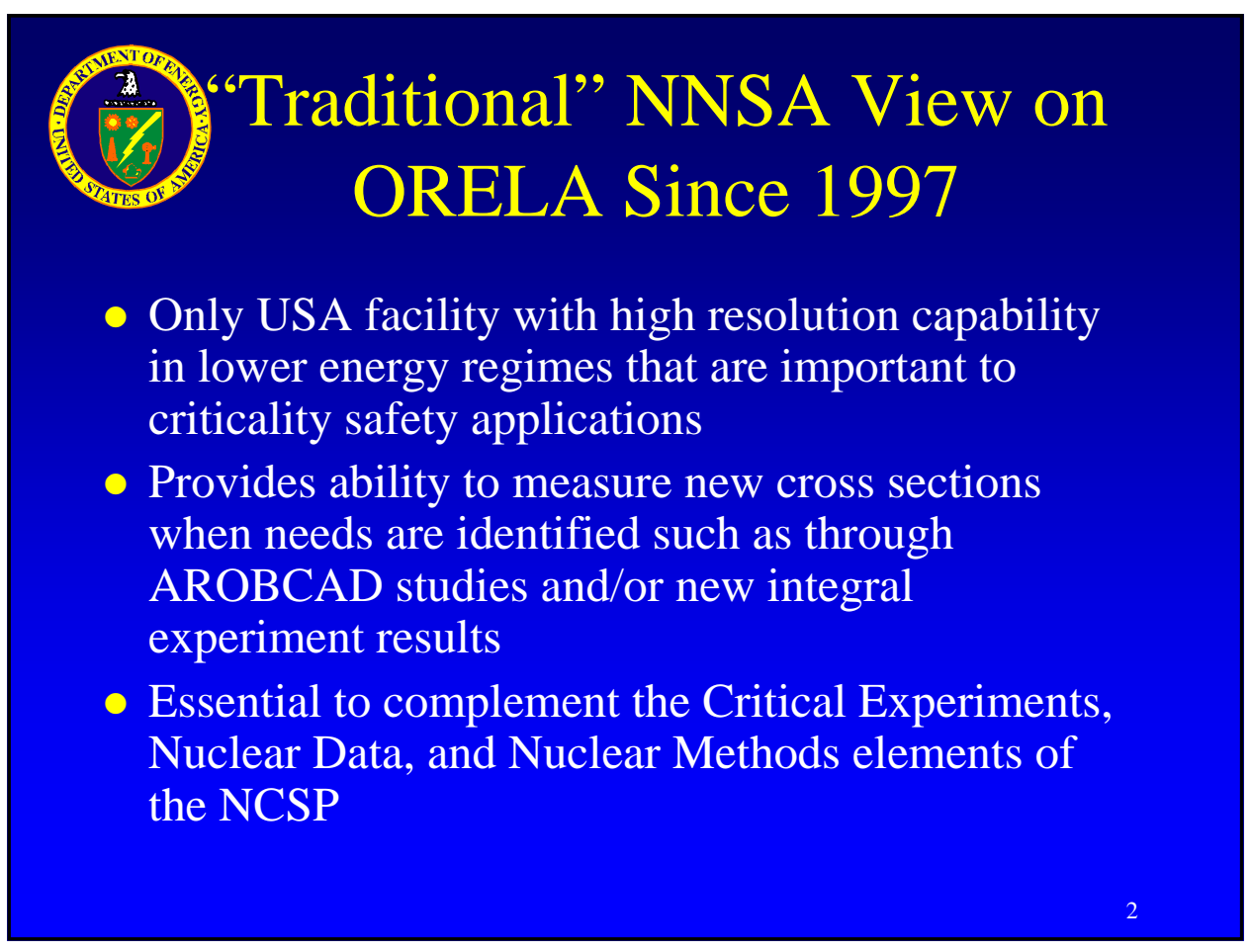




\section{Growing Concerns Regarding ORELA}

- There is a persistent lack of identified measurement needs supporting the NCSP in the out years. Is there an enduring need for new cross-section measurements for criticality safety? Can NDAG and CSEWG efforts alone support our basic NCSP data needs in the future?

- What is the real cost (equipment and personnel) to make ORELA fully functional and reliable and is it justifiable given the apparent absence of the need for new measurements? NNSA is planning to fund $\sim \$ 1.5 \mathrm{M}$ in ORELA upgrades over a three year period. Is that enough?

The Nuclear Criticality Safety Program Infrastructure Supports Criticality Safety Engineers DOE-Wide

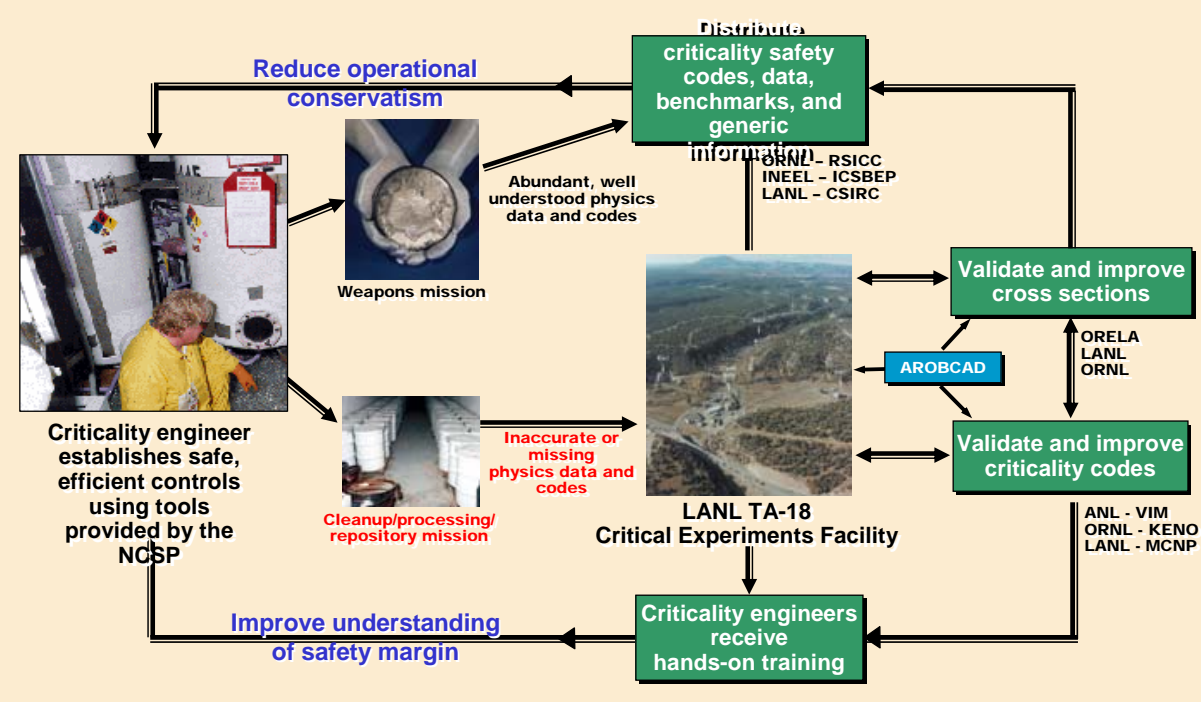




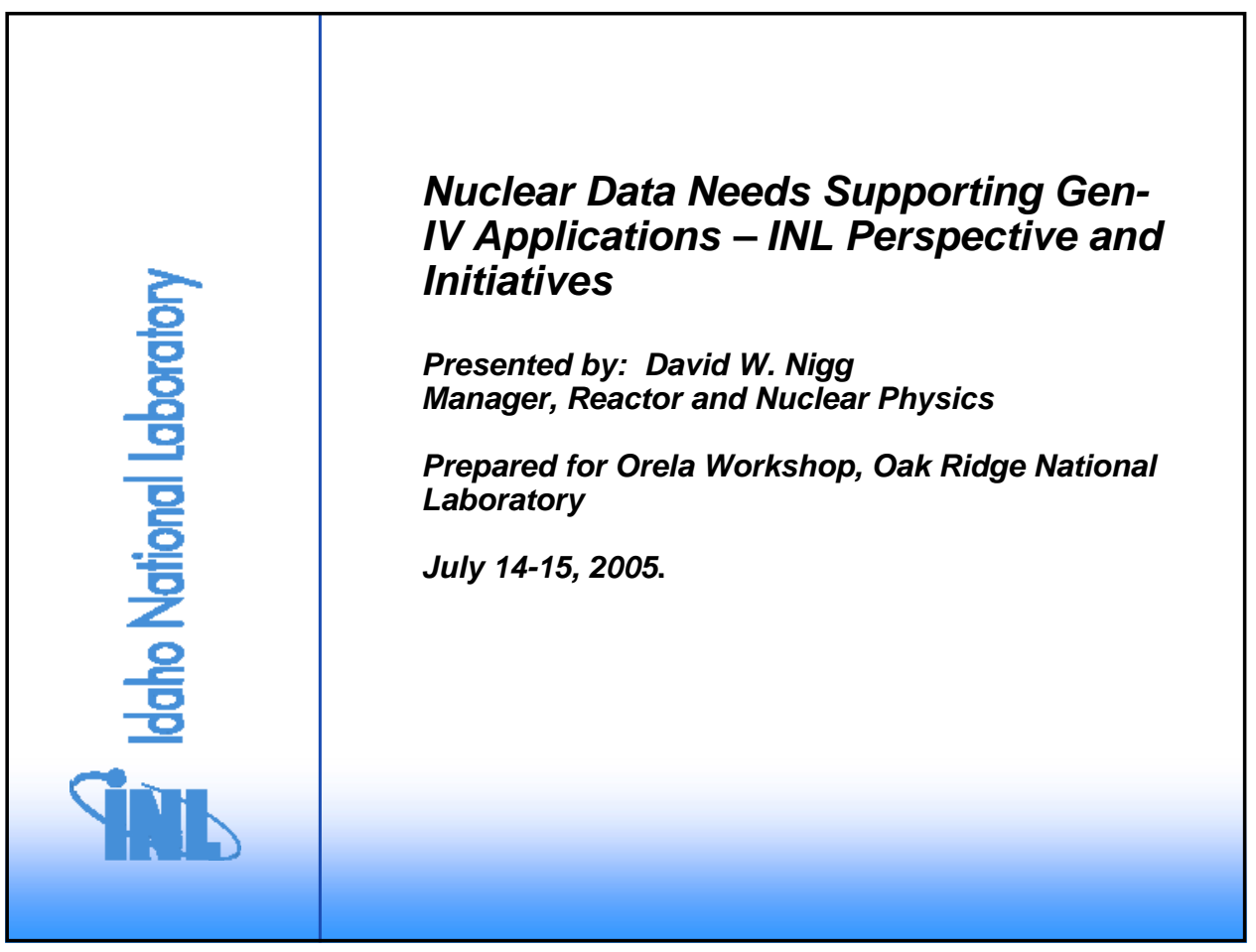

\section{Overview}

- Review of recent US and International workshops on nuclear data needs for Gen-4 and VHTR applications

- Sensitivity Studies to quantify VHTR-related data uncertainty propagation

- INL differential data collaborations

LANL/LANSCE collaboration initiated in FY-05

Nuclear Data Measurements in collaboration with ANL/IPNS 


\section{Generation IV Systems}

\begin{tabular}{|c|c|c|c|c|c|}
\hline System & $\begin{array}{l}\text { Neutron } \\
\text { Spectrum }\end{array}$ & $\begin{array}{l}\text { Fuel } \\
\text { Cycle }\end{array}$ & Size & Applications & $\begin{array}{l}\text { Concept-Specific } \\
R \& D\end{array}$ \\
\hline $\begin{array}{l}\text { Very High Temp. } \\
\text { Gas Reactor (VHTR) }\end{array}$ & Thermal & Open & Med & $\begin{array}{l}\text { Electricity, Hydrogen, } \\
\text { Process Heat }\end{array}$ & $\begin{array}{l}\text { Fuels, Materials, } \\
\mathrm{H}_{2} \text { production }\end{array}$ \\
\hline $\begin{array}{l}\text { Gas-Cooled Fast } \\
\text { Reactor (GFR) }\end{array}$ & Fast & Closed & Med & $\begin{array}{l}\text { Electricity, } \\
\text { Hydrogen, AM }\end{array}$ & $\begin{array}{l}\text { Fuels, Materials, } \\
\text { Safety }\end{array}$ \\
\hline $\begin{array}{l}\text { Lead-alloy Fast } \\
\text { Reactor (LFR) }\end{array}$ & Fast & Closed & $\begin{array}{l}\text { Small to } \\
\text { Large }\end{array}$ & $\begin{array}{l}\text { Electricity, } \\
\text { Hydrogen Production }\end{array}$ & $\begin{array}{l}\text { Fuels, Materials } \\
\text { compatibility }\end{array}$ \\
\hline $\begin{array}{l}\text { Sodium Fast } \\
\text { Reactor (SFR) }\end{array}$ & Fast & Closed & $\begin{array}{l}\text { Med to } \\
\text { Large }\end{array}$ & $\begin{array}{l}\text { Electricity, } \\
\text { Actinide Mgmt. (AM) }\end{array}$ & $\begin{array}{l}\text { Advanced } \\
\text { Recycle }\end{array}$ \\
\hline $\begin{array}{l}\text { Supercritical Water } \\
\text { Reactor (SCWR) }\end{array}$ & $\begin{array}{l}\text { Thermal, } \\
\text { Fast }\end{array}$ & $\begin{array}{l}\text { Open, } \\
\text { Closed }\end{array}$ & Large & Electricity & Materials, Safety \\
\hline $\begin{array}{l}\text { Molten Salt Reactor } \\
\text { (MSR) }\end{array}$ & Thermal & Closed & Large & $\begin{array}{l}\text { Electricity, } \\
\text { Hydrogen, AM }\end{array}$ & $\begin{array}{l}\text { Fuel, Fuel } \\
\text { treatment, } \\
\text { Materials, Safety } \\
\text { and Reliability }\end{array}$ \\
\hline
\end{tabular}

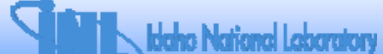

\section{Key System Characteristics Impacted by Nuclear Data}

- Criticality (multiplication factor)

- Reactivity feedback coefficients (e.g., Doppler, Coolant Void)

- Kinetics parameters (e.g., Effective Delayed Neutron Fraction)

- Reactivity loss during irradiation (Excess reactivity)

- Peak power value

- Conversion ratio of sustainable cores

- Transmutation potential of burner cores

- Max dpa, maximum helium- and hydrogen-production, maximum (helium-production)/Dpa

- Decay heat, radiotoxicity, and neutron and gamma radiation levels

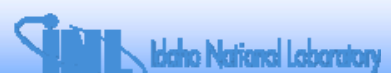




\section{Recent US and International Workshops on Nuclear Data Needs for Gen-IVIVHTR}

- Brookhaven National Laboratory, April 2003

- PHYSOR, April 2004

- DOE/Euratom/AECL International Workshop on Nuclear Data Needs for Gen-IV, Antwerp, Belgium, April 2005

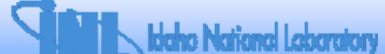

\section{U.S. Gen IV System Design and Evaluation Workshops to Assess Design Capabilities}

- Gen-IV System Design and Analysis Crosscut NTD organized and conducted workshops during 2003 to assess

- Analysis needs for Gen IV systems

- Capabilities of existing analysis tools (computer codes and databases)

- Ongoing work to advance analysis capabilities

- Workshops held in the following topical areas

- Reactor physics design analysis Feb 18-19, 2003 (ANL)

- T-H and safety analysis

- Nuclear data needs

Mar 18-19, 2003 (INEEL) Apr 24-25, 2003 (BNL)

- Workshops attended by lab, university and industry representatives

- Presentations and discussion sessions

- Conclusions and recommendations formulated 


\section{Gen IV Nuclear Data Workshop, April 24-25, 2003, Brookhaven National Laboratory}

- About 30 Participants

- USDOE National Laboratories - ANL, BNL/NNDC, INL, LANL, ORNL

- U.S. Universities - RPI and NCSU

- A working paper on "Nuclear Data for Gen IV," submitted by A. J. Koning and A. Hogenbirk of the Nuclear Research and Consultancy Group, Petten, The Netherlands

- Recommendations for nuclear data needs and path forward

- Summarized experimental facilities in Europe

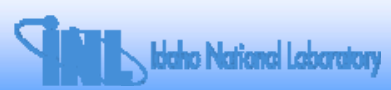

\section{Topical Areas for the BNL Workshop}

- Nuclear Data Needs for Generation IV Nuclear Energy Systems

- Nuclear Data Processing, Applications, and Validation

- Theory and Evaluation

- Nuclear Data Measurements

Acknowledgement - Dr. Temitope Taiwo (ANL) served as the technical secretary for this workshop. The majority of the following material was developed from the comprehensive summary documentation that he produced. 


\section{BNL Workshop Conclusions - Nuclear Data Needs}

- High burnup operation of the VHTR might require re-evaluation of transuranics data (cross sections, decay data, and fission yields). Differential measurements may be needed for selected nuclides

- Fast spectrum systems (GFR, LFR, and SFR) to be used within a closed fuel cycle require additional evaluation of data for transuranics, particularly minor actinides, as well as integral measurements for validation of differential (basic) data and their processing tools

- Non-conventional structural, coolant or fuel-matrix materials may necessitate new evaluations or measurements of basic data

- Systematic approach based on sensitivity and uncertainty analysis required for further specifying data needs. (Need additional covariance data in format suited for analysis)

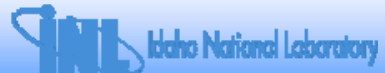

\section{Systematic Approach for Determining Data Needs and Improvement}

- Develop preliminary reactor-core reference configurations along with definitions of major fuel-cycle operation parameters

- Define target accuracies for core and fuel cycle design parameters

- Use sensitivity analysis tools and covariance data to determine the nuclear data uncertainties having greatest impact on performance, safety, and fuel cycle predictions

- Allows sources of uncertainties to be identified

- Enables identification of nuclear data to be improved 


\section{BNL Workshop Conclusions - Nuclear Data Measurements}

- Currently available experimental facilities, equipment, accelerator targets, and personnel required to support necessary differential nuclear data measurements should be able to address the anticipated need for data. Key U.S. facilities identified include those at LANL, ORNL, RPI, and the INL experimental apparatus located at ANL/IPNS

- There should be a strong emphasis on maintenance of the relevant experimental capabilities and on development of a single national collaborative effort, coordinated with relevant international activities, that will provide the necessary information, with appropriate levels of validation, in a manner that makes best use of what will almost certainly be limited financial resources

- A coordinated mechanism should be developed to facilitate the acquisition, maintenance, storage, distribution, and community usage of sample targets, especially purified stable isotopes and actinides. An assessment of the nuclear materials available for this effort should be performed

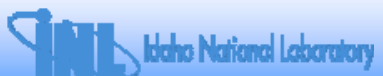

\section{DOE/Euratom/AECL International Workshop on Nuclear Data Needs for Gen-IV}

Antwerp, Belgium, April 5-7, 2005

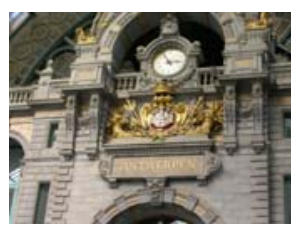

-Follow-on to the two previous workshops with broader international participation: France, Germany, Belgium, Romania, Italy, Netherlands, Russia, USA, Canada, S. Korea, Finland, Czech Republic, Japan, Mexico and various international organizations including IRRM (Geel, Belgium), OECD-NEA, IAEA, etc.

-Discussions of fuel development, intercomparison of evaluations, sensitivity studies, were included with the basic discussions of nuclear data measurements - broad perspective

-Full-Length Proceedings will be published -Some significant consensus on several fronts 


\section{The Antwerp Workshop: Some Key Highlights and Conclusions}

-Data uncertainties and assessment of their impact are a key to improvement of reactor and fuel cycle codes. New approaches to measurement and evaluation will be important in reducing the current uncertainties

-In current fuel cycle scenarios uncertainties in ${ }^{238} \mathrm{U},{ }^{239} \mathrm{Pu}$, some of the higher plutonium isotopes and possibly Americium will be more important than the higher actinides (ANL and CEA Studies)

- Some attention to more accuracy in fission product yields appears to be needed

-Several non-fuel materials (Bi, $\mathrm{Pb}, \mathrm{C}, \mathrm{Si}, \mathrm{Zr}$ ) may need additional improvement.

-Continuing need for better covariance matrices to use in uncertainty studies

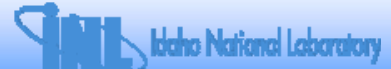

\section{VHTR Uncertainty Assessment} Giuseppe Palmiotti and Temitope A. Taiwo (ANL)

-Study performed by Argonne National Laboratory, at least partially as a direct outcome of recommendations from the Brookhaven workshop.

- Commissioned in connection with the U.S. VHTR effort through INL, but coordinated with broader Gen-IV sensitivity and uncertainty studies underway at ANL (Salvatores, Antwerp, 2005)

-Results have influenced US activities 


\begin{tabular}{|c|c|c|c|c|c|c|c|}
\hline \multicolumn{8}{|c|}{ Proposed Uncertainties - Example } \\
\hline \multirow[b]{2}{*}{ Gr } & \multirow[b]{2}{*}{ Energy } & \multicolumn{6}{|c|}{ Pu240 } \\
\hline & & $v$ & $\sigma_{\mathrm{f}}$ & $\sigma_{\text {inel }}$ & $\sigma_{\mathrm{el}}$ & $\sigma_{\text {capt }}$ & $\sigma_{\mathrm{n}, 2 \mathrm{n}}$ \\
\hline 1 & $150 \mathrm{MeV}$ & 3 & 15 & 45 & 30 & 90 & 100 \\
\hline 2 & $55.2 \mathrm{MeV}$ & 3 & 15 & 30 & 20 & 60 & 100 \\
\hline 3 & $19.6 \mathrm{MeV}$ & 3 & 5 & 15 & 10 & 20 & 100 \\
\hline 4 & $6.07 \mathrm{MeV}$ & 2 & 5 & 15 & 10 & 20 & \\
\hline 5 & $2.23 \mathrm{MeV}$ & 2 & 5 & 15 & 10 & 20 & \\
\hline 6 & $1.35 \mathrm{MeV}$ & 2 & 5 & 15 & 10 & 20 & \\
\hline 7 & $498 \mathrm{KeV}$ & 2 & 5 & 20 & 10 & 20 & \\
\hline 8 & $183 \mathrm{KeV}$ & 2 & 5 & 20 & 10 & 20 & \\
\hline 9 & $67.4 \mathrm{KeV}$ & 2 & 5 & 25 & 10 & 20 & \\
\hline 10 & $24.8 \mathrm{KeV}$ & 2 & 5 & & 10 & 10 & \\
\hline 11 & $9.12 \mathrm{KeV}$ & 2 & 10 & & 10 & 10 & \\
\hline 12 & $2.03 \mathrm{KeV}$ & 2 & 10 & & 10 & 10 & \\
\hline 13 & $454 \mathrm{eV}$ & 2 & 10 & & 10 & 10 & \\
\hline 14 & $22.6 \mathrm{eV}$ & 2 & 10 & & 10 & 10 & \\
\hline 15 & $4.00 \mathrm{eV}$ & 2 & 10 & & 10 & 7 & \\
\hline 16 & $0.54 \mathrm{eV}$ & 2 & 50 & & 5 & 3 & \\
\hline 17 & $0.10 \mathrm{eV}$ & 2 & 50 & & 5 & 2 & \\
\hline
\end{tabular}

\section{Energy Group Structure and Proposed Partial Energy Correlation for the ANL Sensitivity Study}

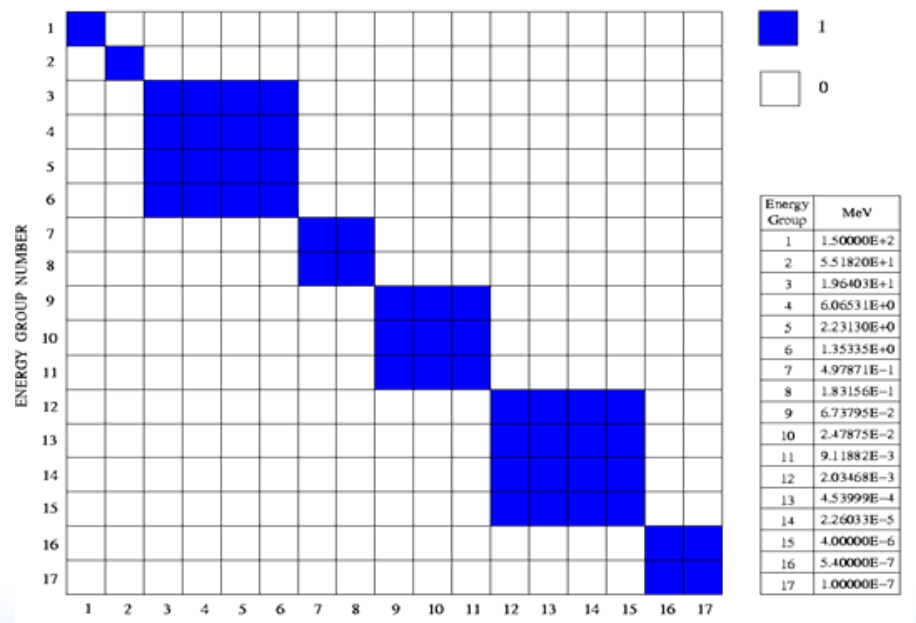

ENERGY GROUP NUMBER 


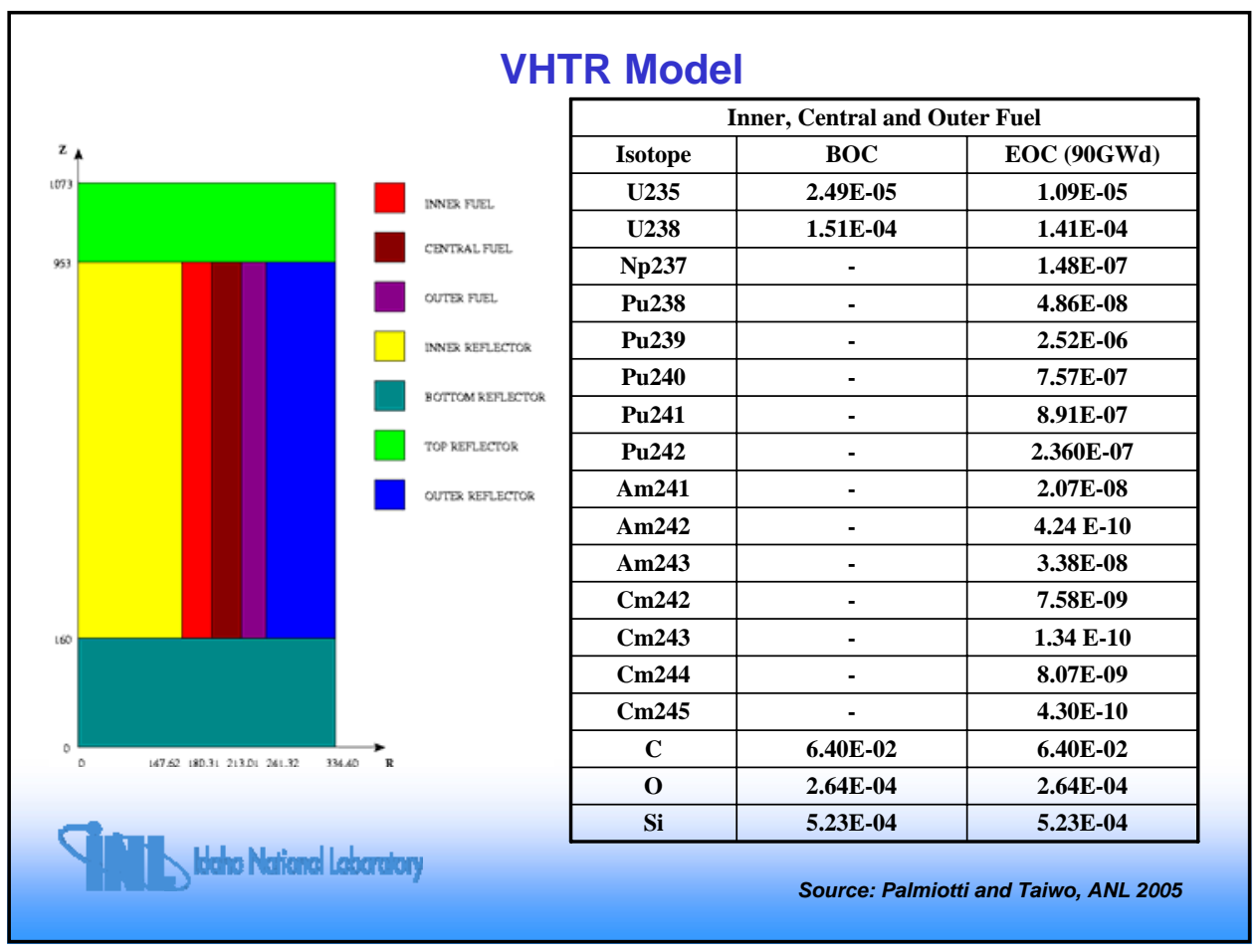

\section{Target Accuracies - ANL VHTR Study}

K-Effective (EOC)

Burnup Reactivity Swing

Peak Power

Reactivity Coefficients

EOC Nuclide Inventories
500 pcm

$1000 \mathrm{pcm}$

$3 \%$

$10 \%$

$5 \%$ 


\begin{tabular}{|c|c|c|}
\hline \multicolumn{3}{|c|}{$\begin{array}{c}\text { Suggested Target Accuracies for Gen-IV } \\
\text { Integral Reactor Parameters (Rimpault et al. } \\
\text { Antwerp, 2005) }\end{array}$} \\
\hline Uncertainties at $1 \sigma$ & System Deve & ment Phase \\
\hline Parameter & Viability & Performance \\
\hline $\mathscr{M} u l t i p l i c$ ation factor, keff & $<0.7 \%$ & $<0.3 \%$ \\
\hline Local power density & $<5 \%$ & $<3 \%$ \\
\hline Structure Damage & $<15 \%$ & $<9 \%$ \\
\hline Reactivity $S$ wing & $(<1.0 \%)$ & $(<0.5 \%)$ \\
\hline Breeding Gain & $<+/-0.06$ & $<+/-0.04$ \\
\hline $\begin{array}{l}\text { Void Reactivity Effect on each component } \\
\text { (leakage; non-leak.) }\end{array}$ & $<16 \%$ & $<10 \%$ \\
\hline Doppler Reactivity Effect & $<16 \%$ & $<10 \%$ \\
\hline De layed $\mathcal{N}$ (eutron Fraction & $<13 \%$ & $<7 \%$ \\
\hline Control Rod Worth & $<16 \%$ & $<10 \%$ \\
\hline$\gamma$ fe ating & $<16 \%$ & $<10 \%$ \\
\hline
\end{tabular}

VHTR Total Uncertainties (\%)

\begin{tabular}{|c|c|c|c|c|c|c|c|c|c|c|}
\cline { 2 - 11 } & $\begin{array}{c}\mathrm{K}_{\text {eff }} \\
\text { BOC }\end{array}$ & $\begin{array}{c}\mathrm{K}_{\text {eff }} \\
\text { EOC }\end{array}$ & $\begin{array}{c}\text { Peak } \\
\text { Power } \\
\text { BOC }\end{array}$ & $\begin{array}{c}\text { Peak } \\
\text { Power } \\
\text { EOC }\end{array}$ & $\begin{array}{c}\text { Doppl. } \\
\text { BOC }\end{array}$ & $\begin{array}{c}\text { Doppl. } \\
\text { EOC }\end{array}$ & $\begin{array}{c}\text { Burnup } \\
{[\text { pcm] }}\end{array}$ & $\begin{array}{c}\text { Decay } \\
\text { Heat }\end{array}$ & Dose & $\begin{array}{c}\text { Neutr. } \\
\text { Source }\end{array}$ \\
\hline $\begin{array}{c}\text { No } \\
\text { Correl. }\end{array}$ & \pm 0.41 & \pm 0.94 & \pm 1.6 & \pm 1.8 & \pm 3.6 & \pm 6.2 & \pm 1653 & \pm 2.5 & \pm 1.9 & \pm 12.2 \\
\hline PEC & 0.58 & 1.07 & 1.9 & 2.1 & 3.1 & 6.1 & 1749 & 3.1 & 2.6 & 14.3 \\
\hline
\end{tabular}

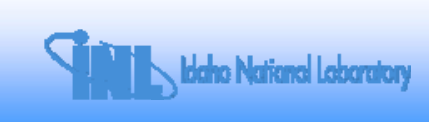

Source: Palmiotti and Taiwo, ANL 2005 


\begin{tabular}{|c|c|c|c|c|c|c|}
\hline \multicolumn{7}{|c|}{$\begin{array}{c}\text { VHTR Uncertainties (\%) PEC - Breakdown by Isotope } \\
\text { (Major Contributions) }\end{array}$} \\
\hline \multirow{2}{*}{ ISOTOPE } & \multicolumn{2}{|c|}{ Keff } & \multicolumn{2}{|c|}{ Doppler } & \multirow{2}{*}{$\begin{array}{c}\text { Burnup } \\
{[\mathrm{pcm}]}\end{array}$} & \multirow{2}{*}{$\begin{array}{l}\text { Neutron } \\
\text { Source }\end{array}$} \\
\hline & вос & EOC & вос & EOC & & \\
\hline U235 & \pm 0.36 & \pm 0.25 & \pm 1.6 & \pm 0.8 & \pm 289 & \pm 0.02 \\
\hline U238 & 0.43 & 0.55 & 2.3 & 2.3 & 139 & 2.61 \\
\hline Pu239 & 0.00 & 0.57 & 0.0 & 3.6 & 656 & 2.26 \\
\hline Pu240 & 0.00 & 0.63 & 0.0 & 4.0 & 1485 & 2.60 \\
\hline Pu241 & 0.00 & 0.17 & 0.0 & 0.5 & 232 & 2.33 \\
\hline Pu242 & 0.00 & 0.02 & 0.0 & 0.1 & 36 & 3.95 \\
\hline Am243 & 0.00 & 0.02 & 0.0 & 0.1 & 26 & 12.60 \\
\hline $\mathrm{Cm} 244$ & 0.00 & 0.00 & 0.0 & 0.0 & 3 & 2.30 \\
\hline
\end{tabular}

VHTR EOC $K_{\text {eff }}$ Uncertainties [pcm]

\begin{tabular}{|c|c|c|c|c|c|c|c|}
\hline Gr. & Energy & $\mathrm{U}^{235} \sigma_{\text {fiss }}$ & $\mathrm{U}^{238} \sigma_{\text {capt }}$ & $\mathrm{Pu}^{239} \sigma_{\text {capt }}$ & $\mathrm{Pu}^{239} \sigma_{\text {fiss }}$ & $\mathrm{Pu}^{240} \sigma_{\text {capt }}$ & $\mathrm{Pu}^{241} \sigma_{\text {fiss }}$ \\
\hline 1 & $19.6 \mathrm{MeV}$ & \pm 0 & \pm 0 & \pm 0 & \pm 0 & \pm 0 & \pm 0 \\
\hline 2 & $6.07 \mathrm{MeV}$ & 1 & 0 & 0 & 0 & 0 & 1 \\
\hline 3 & $2.23 \mathrm{MeV}$ & 1 & 0 & 0 & 1 & 0 & 0 \\
\hline 4 & $1.35 \mathrm{MeV}$ & 2 & 2 & 0 & 1 & 0 & 1 \\
\hline 5 & $498 \mathrm{KeV}$ & 2 & 2 & 0 & 1 & 0 & 1 \\
\hline 6 & $183 \mathrm{KeV}$ & 2 & 2 & 0 & 1 & 0 & 1 \\
\hline 7 & $67.4 \mathrm{KeV}$ & 2 & 4 & 0 & 1 & 0 & 1 \\
\hline 8 & $24.8 \mathrm{KeV}$ & 3 & 7 & 0 & 1 & 0 & 1 \\
\hline 9 & $9.12 \mathrm{KeV}$ & 8 & 10 & 1 & 1 & 0 & 2 \\
\hline 10 & $2.03 \mathrm{KeV}$ & 10 & 24 & 1 & 2 & 1 & 4 \\
\hline 11 & $454 \mathrm{eV}$ & 51 & 185 & 11 & 13 & 11 & 26 \\
\hline 12 & $22.6 \mathrm{eV}$ & 34 & 337 & 11 & 15 & 2 & 56 \\
\hline 13 & $4.00 \mathrm{eV}$ & 38 & 8 & 5 & 12 & 620 & 6 \\
\hline 14 & $0.54 \mathrm{eV}$ & 80 & 9 & 360 & 346 & 24 & 88 \\
\hline 15 & $0.10 \mathrm{eV}$ & 46 & 5 & 21 & 23 & 6 & 23 \\
\hline \multicolumn{2}{|l|}{ Total (pcm) } & 118 & 385 & 361 & 347 & 621 & 110 \\
\hline
\end{tabular}


Current and Required Cross Section Uncertainties (\%) for Target Accuracies

\begin{tabular}{|l|l|c|c|}
\hline Isotope Reaction & Energy Range & Current Uncertainty & Required Uncertainty \\
\hline Pu-240 $\sigma_{\text {cap }}$ & $4 \mathrm{eV}-0.54 \mathrm{eV}$ & 7 & 1.9 \\
\hline Pu-239 $\sigma_{\text {cap }}$ & $0.54 \mathrm{eV}-0.1 \mathrm{eV}$ & 3 & 1.6 \\
\hline Pu-239 $\sigma_{\text {fiss }}$ & $0.54 \mathrm{eV}-0.1 \mathrm{eV}$ & 3 & 1.3 \\
\hline $\mathrm{U}-238 \sigma_{\text {cap }}$ & $22.6 \mathrm{eV}-4 \mathrm{eV}$ & 3 & 1.6 \\
\hline $\mathrm{U}-238 \sigma_{\text {cap }}$ & $454 \mathrm{eV}-22.6 \mathrm{eV}$ & 3 & 2.2 \\
\hline Pu-241 $\sigma_{\text {fiss }}$ & $22.6 \mathrm{eV}-4 \mathrm{eV}$ & 10 & 7.4 \\
\hline $\mathrm{C} \sigma_{\text {inel }}$ & $19.6 \mathrm{MeV}-6.07 \mathrm{MeV}$ & 30 & 15 \\
\hline $\mathrm{Si} \sigma_{\text {cap }}$ & $0.54 \mathrm{eV}-0.1 \mathrm{eV}$ & 20 & 12 \\
\hline $\mathrm{C} \sigma_{\text {cap }}$ & $4 \mathrm{eV}-0.54 \mathrm{eV}$ & 20 & 14 \\
\hline $\mathrm{C} \sigma_{\text {cap }}$ & $19.6 \mathrm{MeV}-6.07 \mathrm{MeV}$ & 20 & 21 \\
\hline $\mathrm{C} \sigma_{\text {inel }}$ & $6.07 \mathrm{MeV}-2.23 \mathrm{MeV}$ & 35 & 7.1 \\
\hline $\mathrm{U}-236 \sigma_{\text {cap }}$ & $22.6 \mathrm{eV}-4 \mathrm{eV}$ & 8 & 9.4 \\
\hline Am-241 $\sigma_{\text {cap }}$ & $0.54 \mathrm{eV}-0.1 \mathrm{eV}$ & 10 & 11 \\
\hline Am-243 $\sigma_{\text {cap }}$ & $4 \mathrm{eV}-0.54 \mathrm{eV}$ & 20 & 14 \\
\hline
\end{tabular}

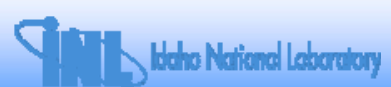

Source: Palmiotti and Taiwo, ANL 2005

\section{Conclusions - ANL VHTR Study}

- The VHTR uncertainty analysis has indicated the need for cross section improvement for specific isotopes, reaction rates, and energy ranges, mostly in the resolved resonance domain

- The uncertainty analysis is strongly influenced by the covariance matrix (both diagonal and correlation values). Use of more scientific based values will help to better assess uncertainties

- Target accuracies on cross sections has shown needs that in some cases will be difficult to achieve both in measurements and/or evaluations

- Integral experiments and statistical data adjustments are likely to play a useful role in overcoming most of the difficulties 


\section{INL Nuclear Data Initiative for VHTR and Other Advanced Reactor Physics Applications}

- Builds on historical INL capabilities in nuclear physics and radiochemistry

- Designed to complement related efforts elsewhere, domestic and international, with a focus on Gen-IVINGNP and AFC. Activities are coordinated through the recently-organized AFC/Gen-IV Physics and Nuclear Data Working Group

- Three basic technical components:

-In-core integral benchmark experiment evaluation and leadership of OECD/NEA IRPhEP

-Support of measurements planned at LANL under AFCI (LANL collaboration ongoing) -Differential nuclear data measurements Gen-IV (ANL collaboration)

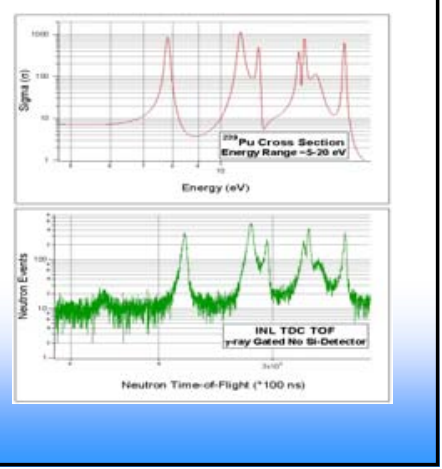

\section{Differential Nuclear Data Measurements - Current INL Collaborations}

- Measurements at LANL/LANSCE under AFC support. INL began assisting with these measurements in FY-05.

- Proof of Principle experiment for complementary measurements focused on Gen-IVINGNP needs using new methods that offer improved accuracy and precision are being conducted by INL in collaboration with ANL/IPNS. Initial data are now available. 


\section{INL-LANSCE Collaboration (AFCI) - Differential Cross Section Measurements}

- Produce targets to perform (n, $\gamma)$ experiment on ${ }^{239} \mathrm{Pu}$ and ${ }^{242} \mathrm{Pu}$ Currently of interest to $A F C l$ and NGNP

- Approximately $1 \mathrm{mg} P u$ Electroplated on thin $\mathrm{Ti}$ backing.

- DANCE array

- LDRD funding has allowed development of basic target fabrication approach using surrogate materials.

- AFC program funding received Oct 1, 2005.

- Initial target delivery date June 2005.

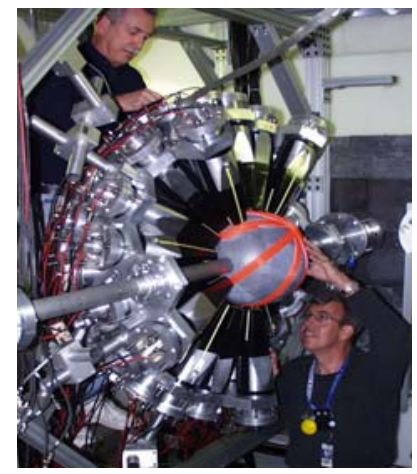

- Follow on activities anticipated (targets for ${ }^{235} \mathrm{U}$ fission measurements, 240Pu etc.)

DANCE Array. Photo courtesy of J. UlImann, LANL

\section{INL Actinide Target Preparation - Process Development using Gd Surrogate}

J.D. Baker, C.A. McGrath, C.L. Riddle, A. Caffrey (student)

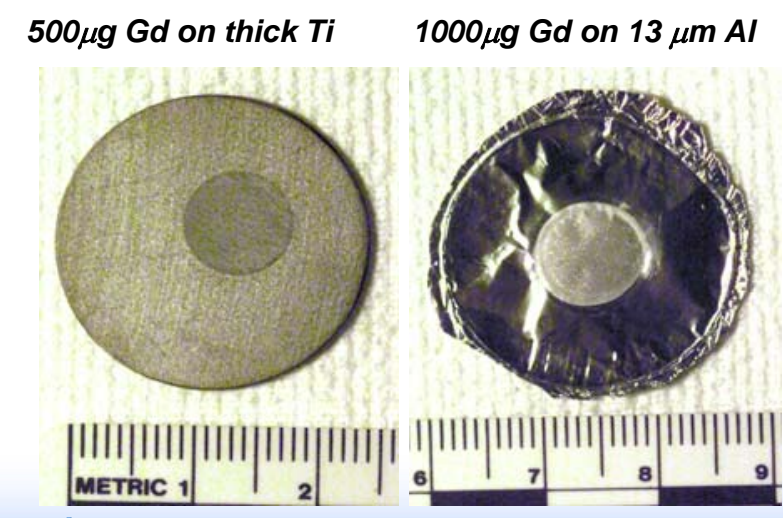

$\sim 100 \mu g$ Gd on $2 \mu \mathrm{m} \mathrm{Ni}$

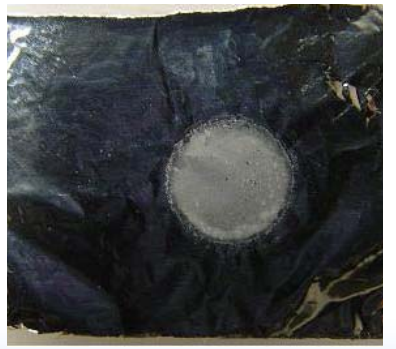




\section{${ }^{239} \mathrm{Pu}$ Target - Delivered June 2005}

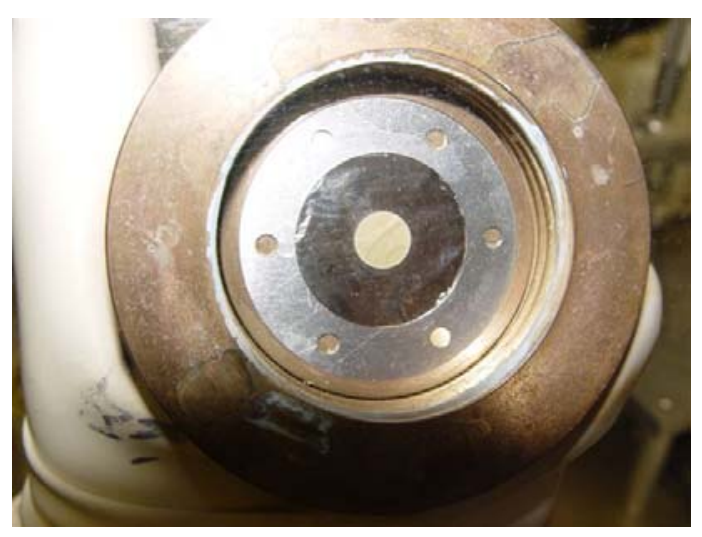

Note: ${ }^{242} \mathrm{Pu}$ Target to be delivered later in

\section{INL-ANL/IPNS Collaboration \\ J.D. Cole, M.W. Drigert, R. Aryaeinejad, C. Wemple (INL)} R.V.F. Janssens, B.J. Micklich (ANL)

- INL apparatus at the Argonne Intense

Pulsed Neutron Source (IPNS) uses

successful methods from low-energy nuclear

physics studies of the fission process via coincidence techniques.

- The same techniques, applied to cross section measurements, offer a new approach to this type of measurement with potential improved accuracy, compensation for target impurities, improvement of resolution, and very effective background suppression.

- FY04 and early FY-05: System reconfiguration for absolute cross section measurements; International peer review of INL proposal.

- FY-05: Proof of principal experiment initiated during the February-June 2005 16week IPNS run cycle. The effort is currently underway and initial results have been obtained.

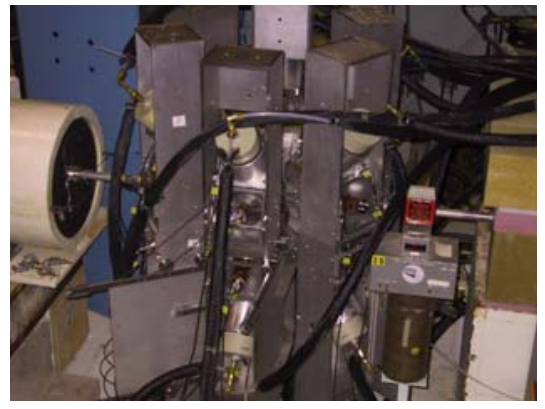

IPNS Detector Array. 


\section{ANL/IPNS Setup}
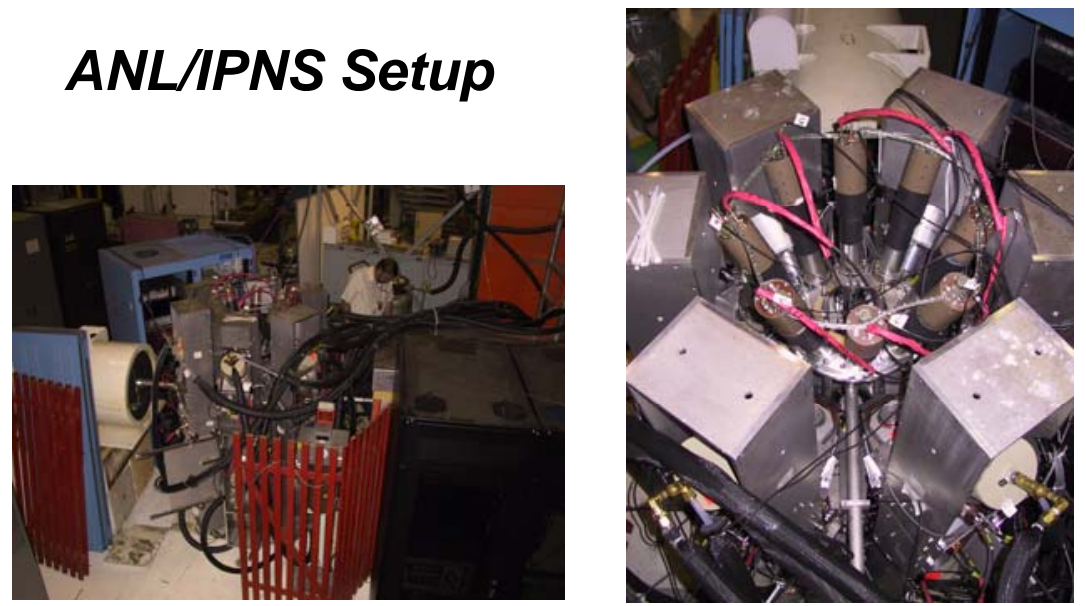

The detector array is composed of Compton suppressed HPGe gamma detectors as well as liquid scintillation neutron detectors, operating in coincidence mode.

\section{ANL/IPNS is an intense, well characterized source of neutrons}

- Spallation neutron source

- $450 \mathrm{MeV}$ pulsed proton beam

- $\approx 3 \times 10^{12}$ protons per pulse

- 30 pulses per second

- $\approx 70$ ns proton full pulse width (Lujan width is $\sim 250 \mathrm{~ns}$ )

- Similar to Lujan in terms of intensity, but energy resolution is improved at IPNS due to shorter pulse width.

- INL has excellent beam access for very long runs (100s of hours) required by coincidence technique.

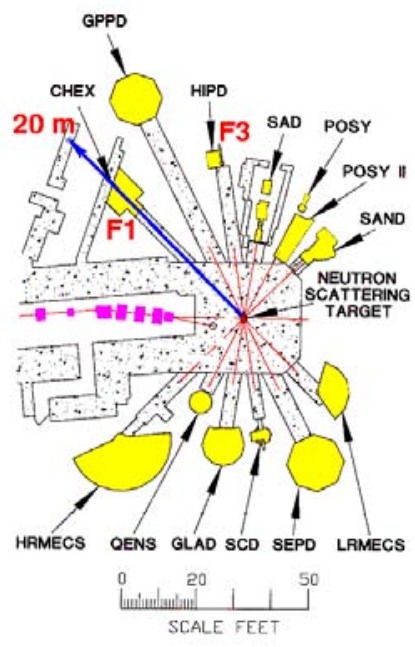




\section{IPNS Neutron Spectrum}

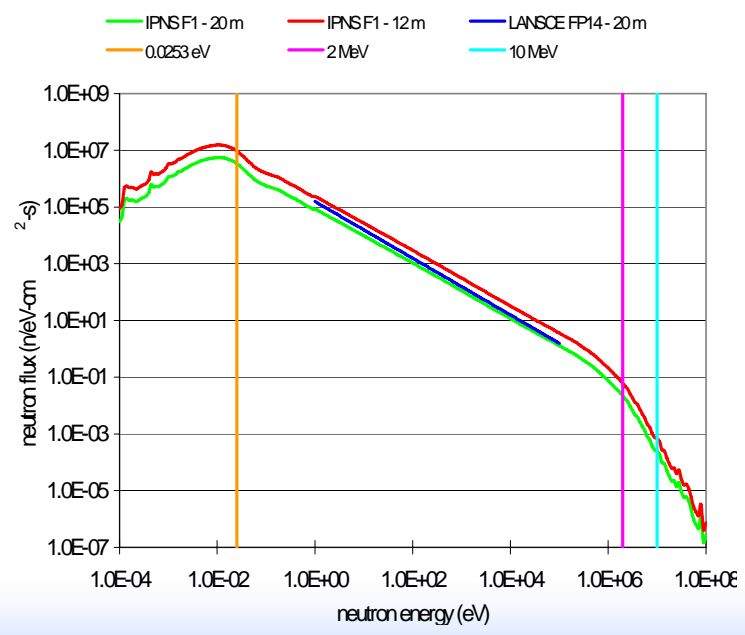

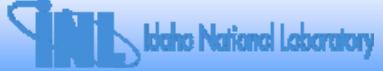

IPNS Beam Line Shielding

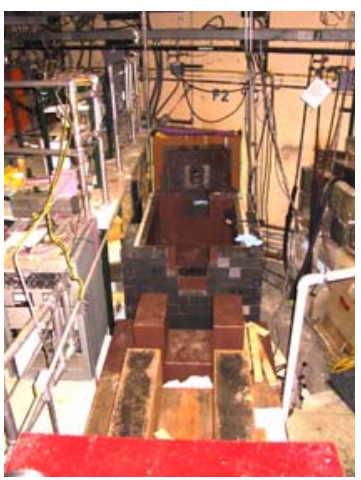

Time-of flight fission chamber has been installed in the INL beamline for absolute flux measurements

\section{bato Natianal Laboritary}

IPNS Target Position

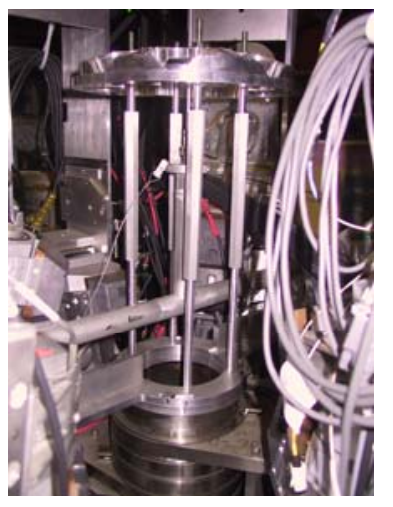

Shipment of ${ }^{239} \mathrm{Pu}$ targets

matched to silicon fission

detectors - April 2005. These

detectors provide an additional

coincidence trigger. 
Compton-suppressed

germanium detectors have

been repaired and annealed

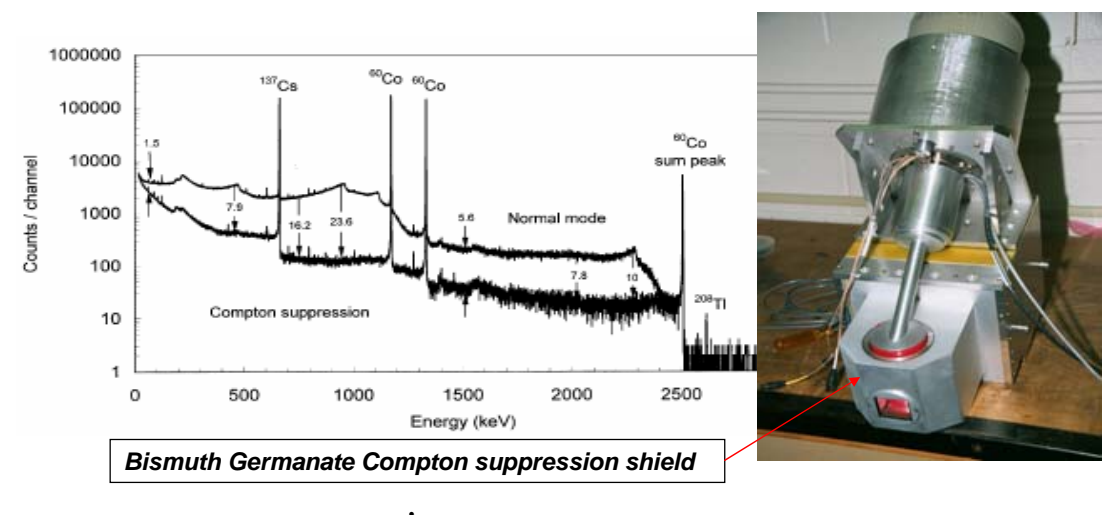

Whoto Ntiand laburdory

Time of Flight Fission Chamber

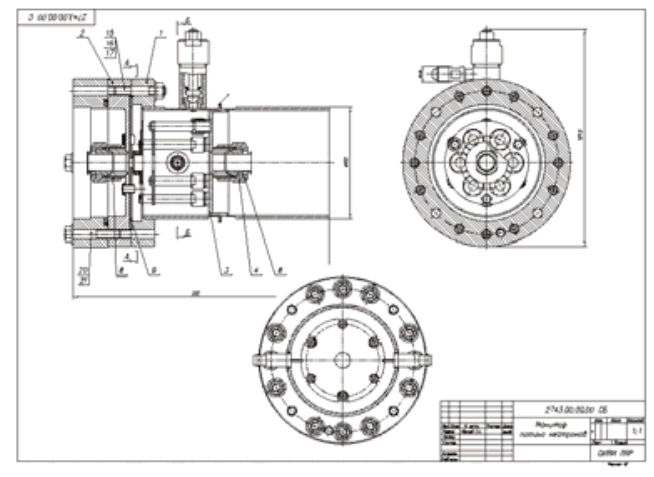

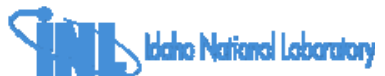


Information is obtained for all observable interactions simultaneously in a single data set over the entire accessible energy range and sorted later

- Cross sections

- $n$,Fission

- $n, \gamma$

- $n, n^{\prime}$ (Low-Energy)

- For isotope pairs
- $\quad$ Prompt fission product yields

- By isotope pair

- By element

- By mass chain

- $\gamma$-ray Yields

- Neutron \& $\gamma$-ray Multiplicities \& Distributions

Note: Capture cross section requires a nuclear level scheme. This should be obtainable using $\gamma-\gamma$ coincidence data.

\section{ENDF-6.8 Evaluation of ${ }^{239}$ Pu Fission Cross Section}

(Source: NEA/Janis 2.1)

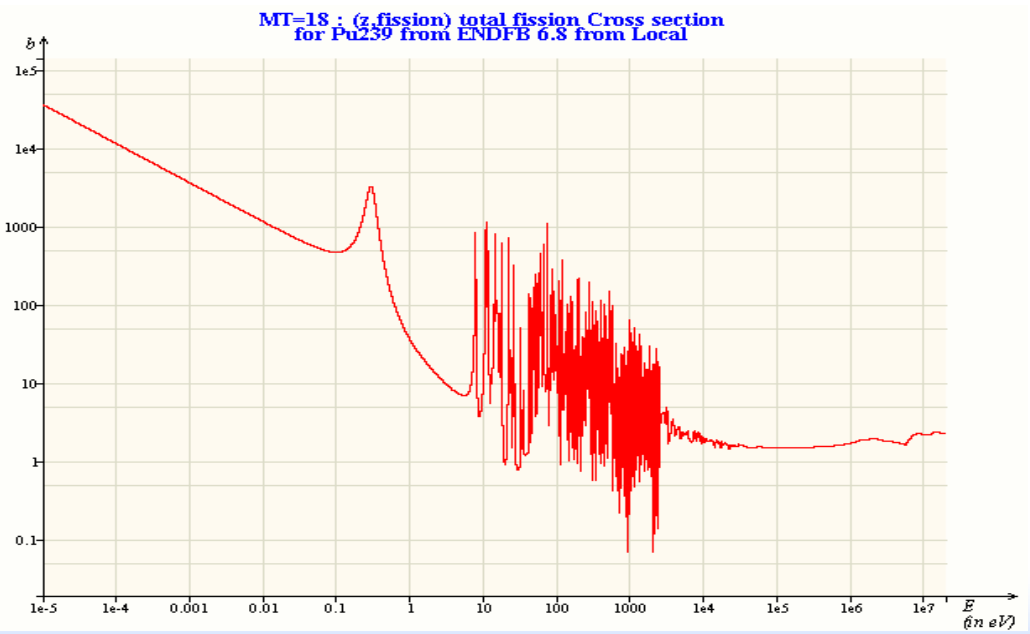

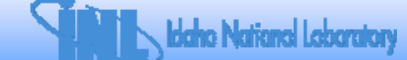


Initial Time of Flight Correlated ${ }^{239} \mathrm{Pu}$ Fission Event

Spectrum (Si Detectors). IPNS, May-June 2005

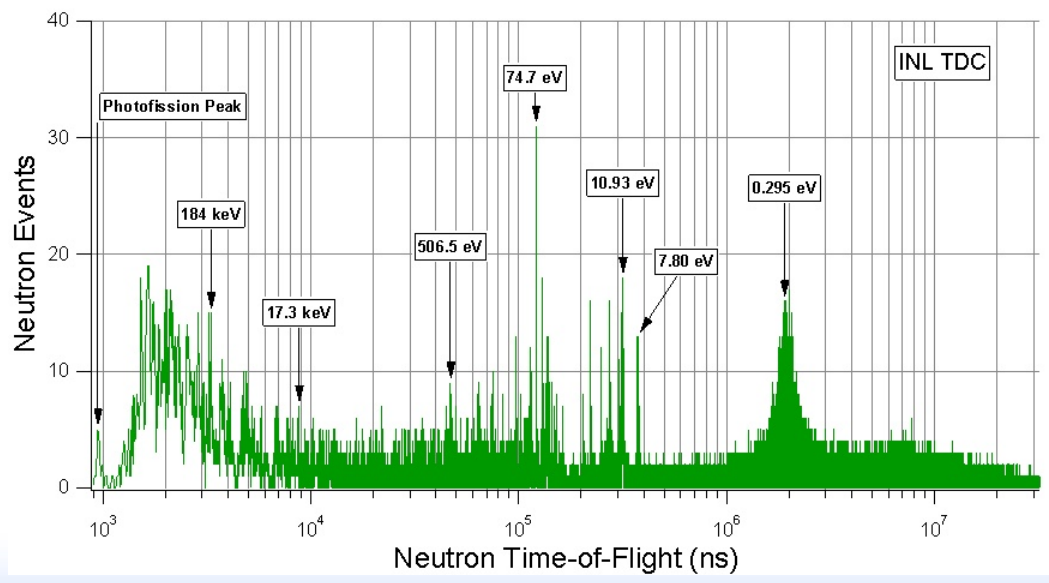

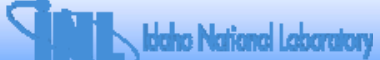

Initial Time of Flight Correlated ${ }^{239} \mathrm{Pu}$ Fission Event Spectrum (Si Detectors). IPNS, May-June 2005 Preliminary Low-Energy Line Identification

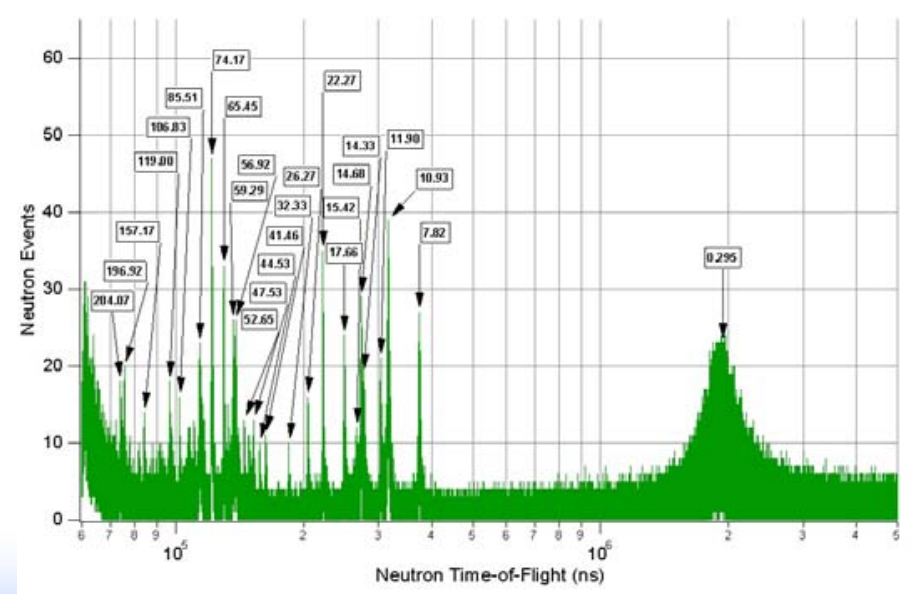




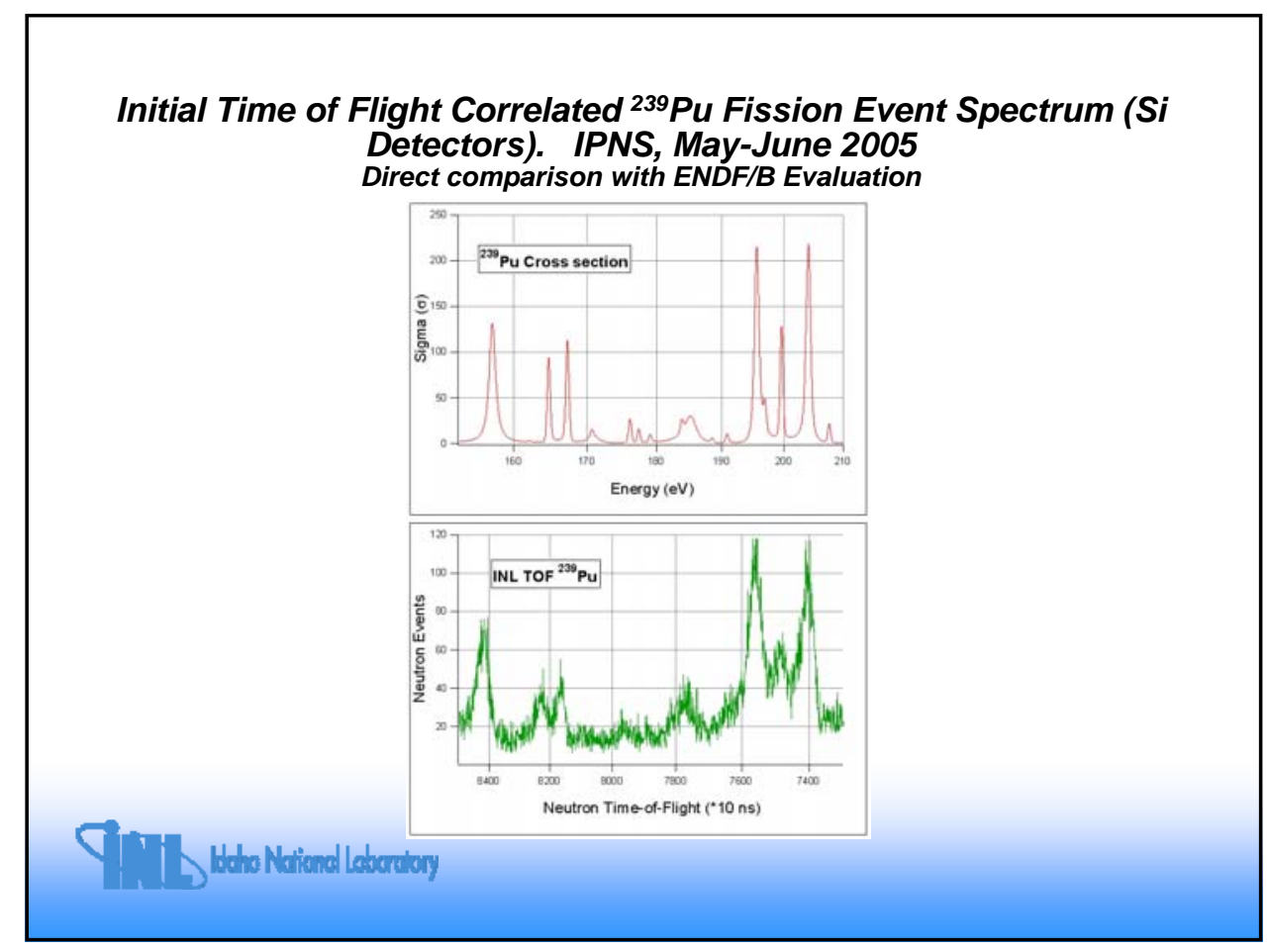

Initial Time of Flight Correlated ${ }^{239} \mathrm{Pu}$ Capture-Fission Anticoincidence Event Spectrum. IPNS, May-June 2005

Direct comparison with ENDF/B Evaluation
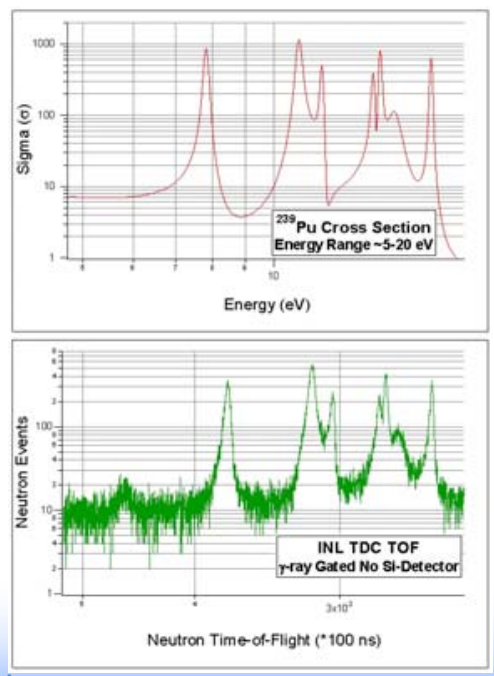

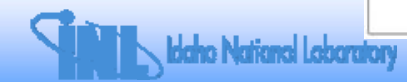




\section{Key Strengths of Coincidence Approach}

- Noise (background radioactivity) suppression

- Self-consistent separation of competing reactions

- Suppression of target contaminant interactions

- Potential for resolution of resonances independent of accelerator pulse width

- Offers capabilities complementary to those of LANSCE, Orela, RPI, European facilities etc.

$\rightarrow$ Net Result: Improved Accuracy

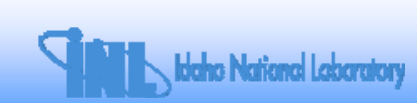

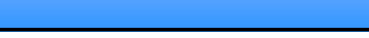

\section{Targets for IPNS Measurements}

- Collaborating with the nuclear physics group at Flerov Laboratory of Nuclear Reactions at the Joint Institute of Nuclear Research, Dubna, Russia, and "sharing" some of the more difficult targets to obtain.

- Initial planned target acquisition includes ${ }^{239} \mathrm{Pu}$ calibration targets matched to Si fission detectors for the proof of principal experiment.

- Additional potential future targets available include ${ }^{240} \mathrm{Pu},{ }^{241} \mathrm{Pu}$, ${ }^{242} \mathrm{Pu},{ }^{242 \mathrm{~m}} \mathrm{Am},{ }^{236} \mathrm{~Np},{ }^{243} \mathrm{Am},,{ }^{242} \mathrm{Cm},{ }^{243} \mathrm{Cm},{ }^{244} \mathrm{Cm},{ }^{250} \mathrm{Cm}$, and ${ }^{247} B K$. 


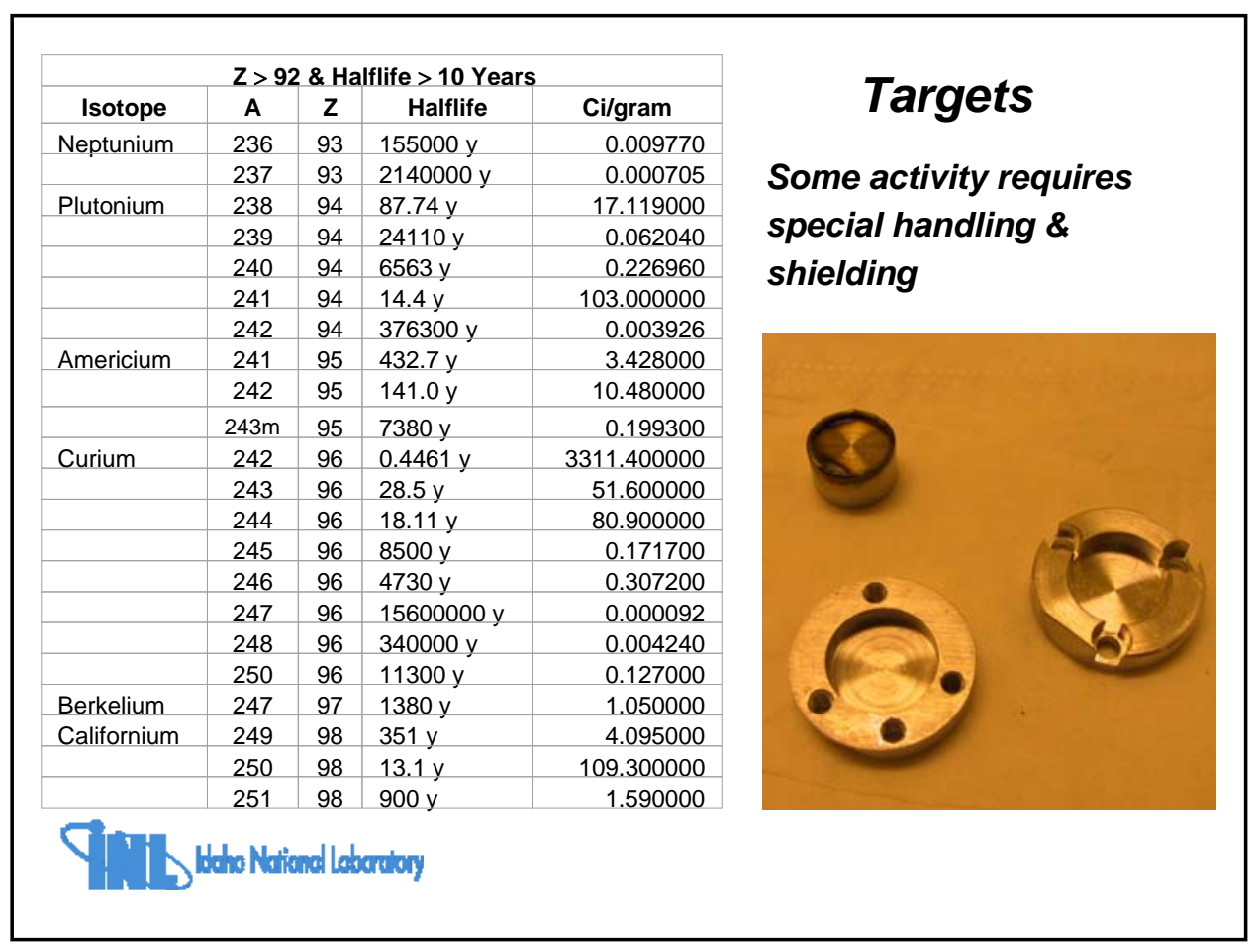

\section{Wrap Up}

- Near-term nuclear data improvement needs for Gen-IV are continuing to come into focus as a result of the various efforts to address the issue

- A three-pronged approach including updated evaluations, additional integral experiments, and additional differential measurements using complementary techniques appears to be emerging.

- Contrary to what one might first think, some of the more common actinides and non-fissionable structural materials appear from current studies to be in need of additional near term attention

- In the longer term, data for the minor actinides will also likely require some improvement as the more exotic fastspectrum reactor concepts come to the forefront. 



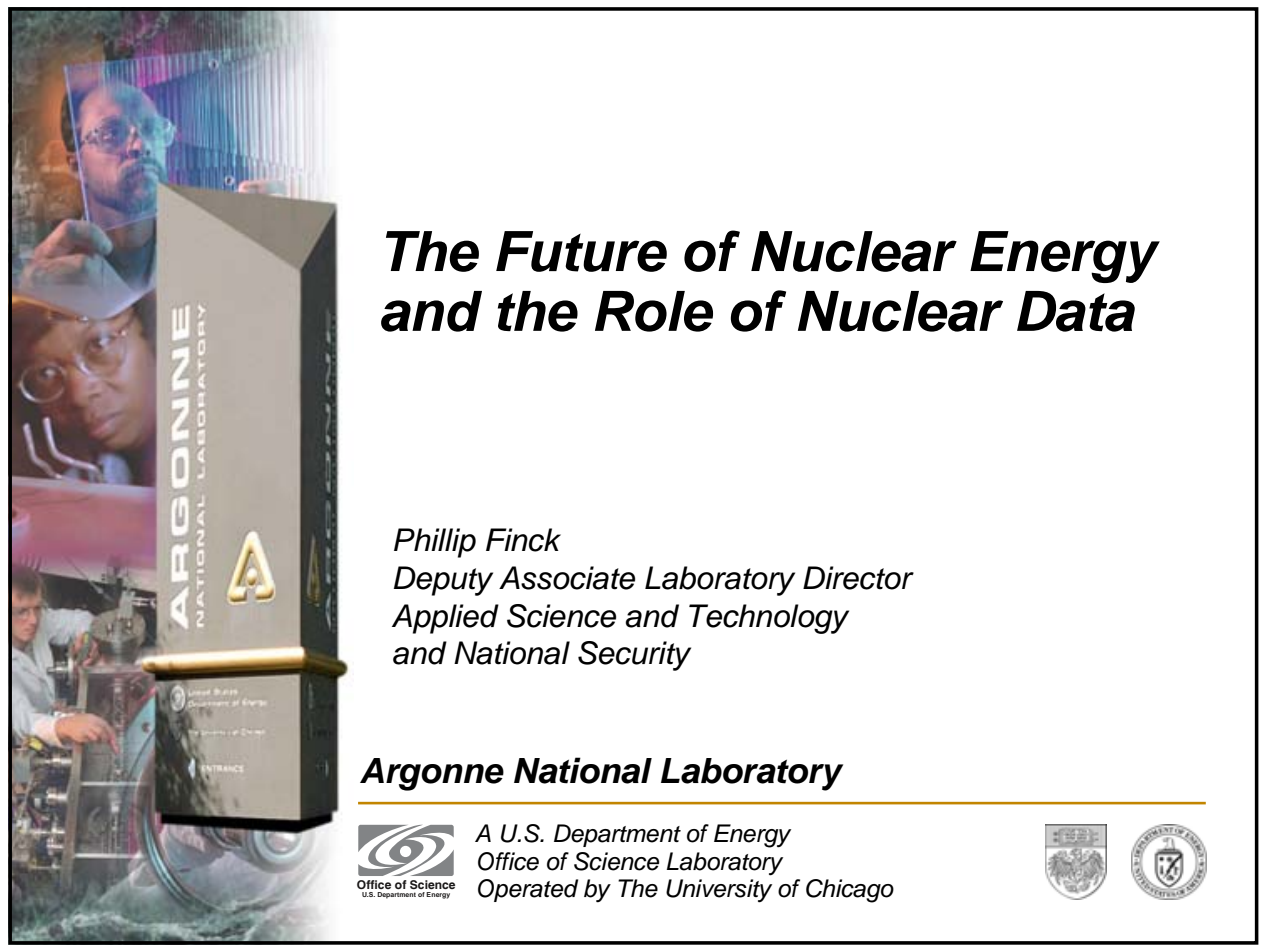

\section{Background}

- Possible energy crisis due to growing demand

- Possible environmental crisis due to greenhouse gases

- The current energy/environment model is not sustainable

- Nuclear energy has a key role to play for the future of mankind

- Many future nuclear technologies will derive from current technologies

- Nuclear data needs need to be derived in a systematic way 


\section{Why Should Nuclear Energy Play A Major Role?}

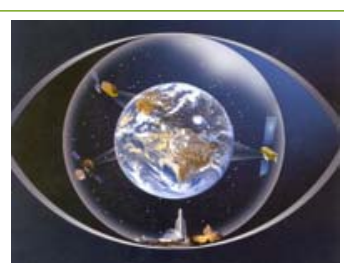

- No $\mathrm{CO}_{2}$ emissions and no contribution to Global Warming

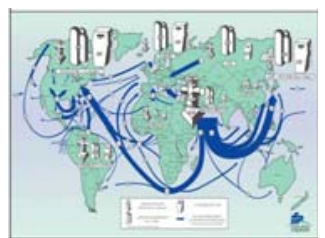

- Enhances the energy supply security

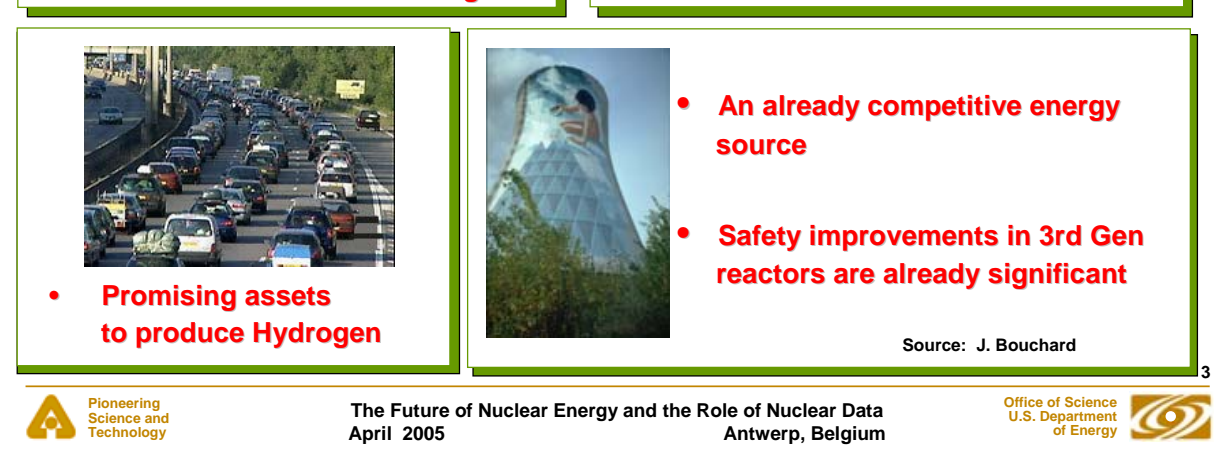

\section{Energy Demand}

- There is a potential for massive growth in energy demand

- Factor of several by 2100

\section{- Fueled by:}

- Population growth in developing countries

- GDP growth in developing countries

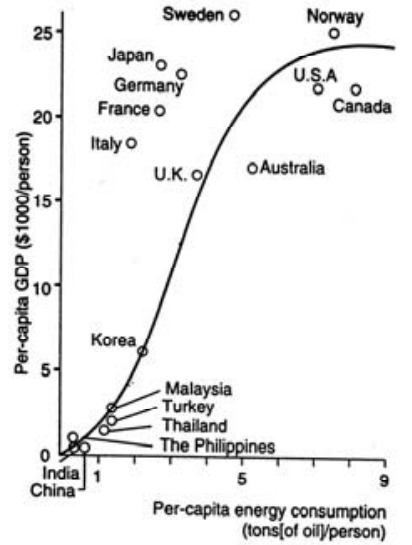

Fig. 1 Energy Use vs GDP [3] 


\section{Energy Usage}

- Primary energy use is divided in three roughly equal parts (OECD)

- Electricity

- Transportation

- Heating, industrial, and agricultural applications

- Nuclear makes heat, and can be applied to all three sectors

- Hydrogen for transportation

- Hydrogen and electricity are complimentary

- Nuclear is a clean way of producing hydrogen, but needs to be proved efficient

- Nuclear for potable water

- Growing issue

- Source of local conflict

- Global market: developed and developing countries

\section{Key Issues for Nuclear Energy Development}

\section{$\longrightarrow$ Economics}

$\longrightarrow$ Safety

$\longrightarrow$ Proliferation

$\longrightarrow$ Waste

$\rightarrow$ Supplies of Uranium Sustainability 


\section{Estimated Stockpile of Spent Fuel (MTIHM)}

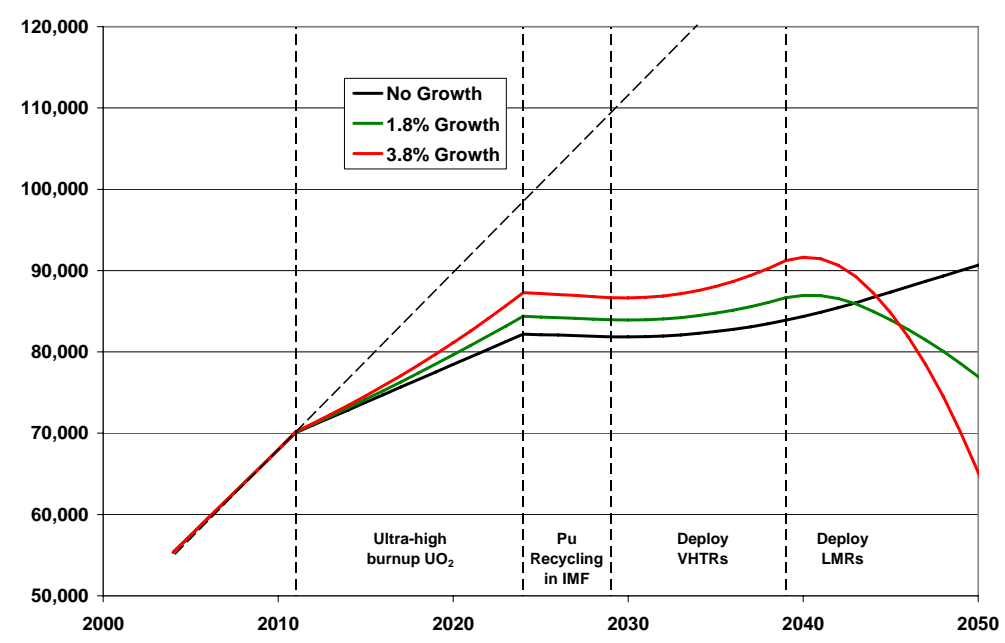

\section{GENERATION IV}

Nuclear energy systems deployable no later than 2030 in both developed and developing countries, for generation of electricity and other energy products

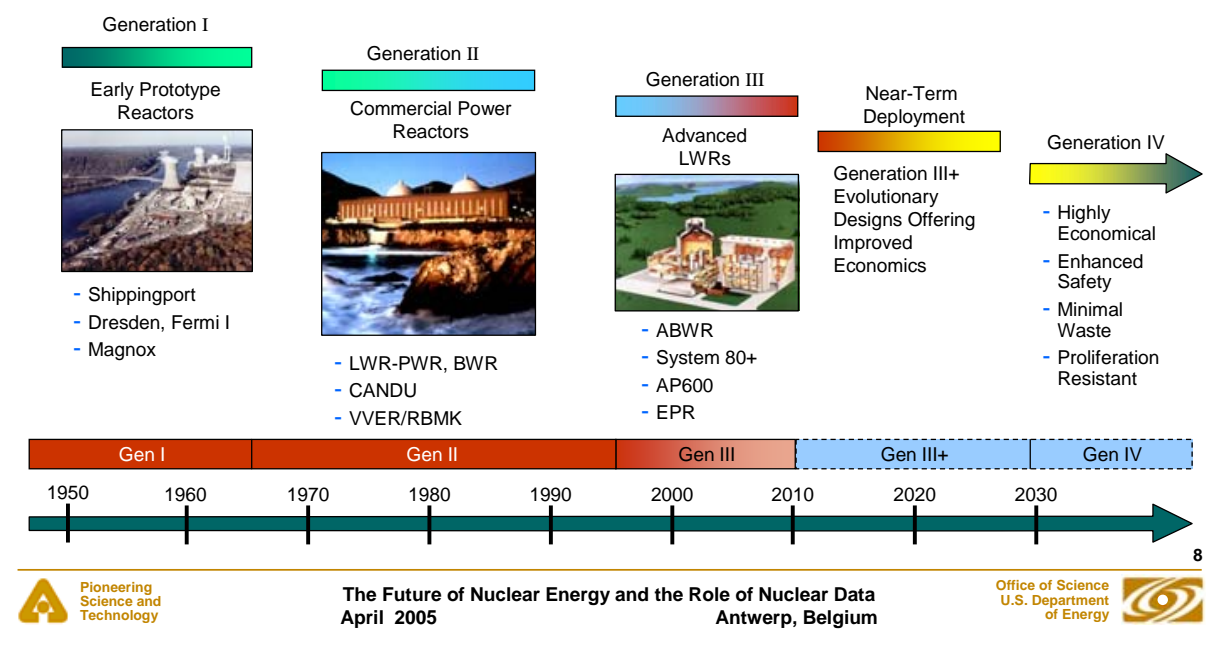


The Role of Nuclear Data in Developing Future Generation Nuclear Energy

Some examples have been studied using a simplified approach:

- Extended Burnups for LWR's

- Very High Temperature Reactors

- Fast Reactors: GFR, SFR, LFR

- Fuel Cycle Performance

- Accelerator Driven Systems

Uncertainties strongly depend on covariance data used!

\section{Note on uncertainty estimates}

- Credible and complete covariance data were not available

- Used estimates of these data

- Uncertainties were propagated through standard static and depletion codes

- Results should not be used in an absolute sense, but have a relative value 


\section{Extended Burnup for LWR's}

- Fuel Burnups in LWR's have been slowly increasing in order to reduce costs

- Current plans indicate that burnup might be increased in the [50-100] GWd/ton range

- As burnup increases, the neutronic contributions of transuranics become predominant
- Uncertainties on $\mathrm{K}_{\infty}$ at Beginning of Cycle (BOC)

Total uncertainty is $\sim 510 \mathrm{pcm}$ (U235: 350 pcm; U238: 360 pcm)

- Uncertainties on $\mathrm{K}_{\infty}$ at End of Cycle (EOC 100GWd/ton) Total uncertainty is $-1220 \mathrm{pcm}$ (Pu239: 620 pcm; Pu241: 320 pcm; Pu 240: 620 pcm; U238: 690 pcm)

- Uncertainty on Burnup Swing $\sim 2240$ pcm, dominated by Pu240

- Uncertainties on isotopics Largest ( 6\%) on Np237 Pu238 Pu240 $\mathrm{Am}$ and $\mathrm{Cm}$ isotopes

Due to capture of U236, Pu240, Am241, 243 and $\mathrm{Cm} 242,244$

\section{The Very High Temperature Reactor}

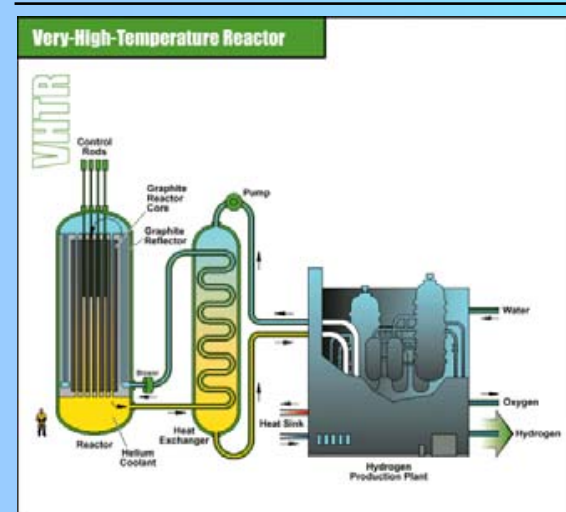

Graphite moderated

U235 enrichment > 10\%

Very High Burnup

Very High Thermal Efficiency

High Outlet Temperature
- Uncertainties on $\mathrm{K}_{\infty}$ at $\mathrm{BOC}$

Total uncertainty is $-580 \mathrm{pcm}$ (U235: 360 pcm; U238: 430 pcm)

- Uncertainties on $\mathrm{K}_{\infty}$ at EOC Total uncertainty is $\sim 1070 \mathrm{pcm}$ (Pu240: 630 pcm; Pu239: 570 pcm; U238: $550 \mathrm{pcm}$ )

- Uncertainty on Burnup Swing $\sim 1749 \mathrm{pcm}$, dominated by Pu240

Note: graphite $S(\alpha, \beta)$; lower resonances of MA's if in a burner mode 


\section{Fast Reactors}

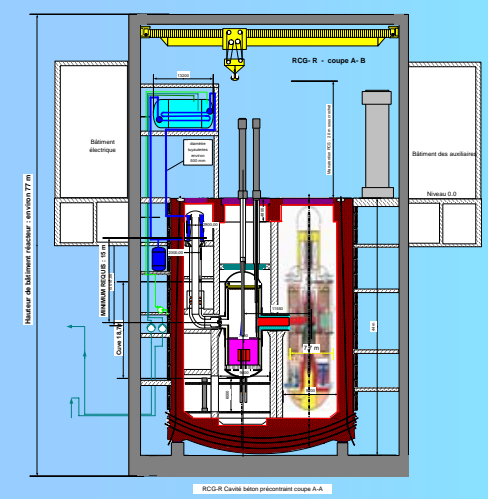

Breeder or Burner
- Uncertainties on $\mathrm{K}_{\infty}$ : 2000pcm

U238 (inelastic, capture), Pu239 and Pu241 (fission) still predominate but $\mathrm{Si}$ (inelastic) contributes $430 \mathrm{pcm}$ in GFR

- Uncertainties on void worth: -12$20 \%$

He void worth: small absolute value < uncertainties. Sign is unclear

- Uncertainty on Doppler worth: -5$10 \%$

- Uncertainty on Burnup Swing: less than $1000 \mathrm{pcm}$

Fuels, structures, and reflectors

might contain new materials ( $\mathrm{Zr}, \mathrm{Si})$

\section{Nuclear data needs}

- As compared to current concepts, these examples show a slight increase in overall uncertainties

- They do not indicate an urgent need for large amounts of new data; only isolated data might be needed urgently

- But we must recall that predictive codes for existing reactors achieve very low uncertainty, not because of the quality of the nuclear data, but thanks to a series of "adjustments" to their data libraries

- These adjustments were made possible by the accumulation of integral data from measurements in reactors and mockup facilities. These were lengthy and costly, and might not be available in the future. 


\section{Nuclear Data Needs (2)}

- The need to reduce costs and R\&D schedules will lead us to use powerful simulation techniques to (partially) replace experiments:

- Better codes

- Better data:

- Reduce uncertainties on key principal reactions.

- Provide reliable, complete, and systematically derived covariance files. These are absolutely key.

- Need a few "clean" integral experiments (simple, well documented...)

- Are we equipped to do that?

\section{Conclusions}

- Nuclear has key role in future energy supply

- Specific nuclear data needs for existing and new concepts

- Covariance files are needed to move the reactor design community towards a more efficient $R, D$ \& D model

- "Quick and dirty" approaches are not adequate

- Need a systematic approach for defining needs

- Need a systematic approach for fulfilling needs

- The "easy" measurements are behind us; we now have to face the tough ones 
NNDC Perspective on Nuclear

\section{Reaction Databases and ORELA}

\section{Pavel Oblozinsky}

National Nuclear Data Center

Brookhaven National Laboratory

\section{Nuclear Reaction Databases}

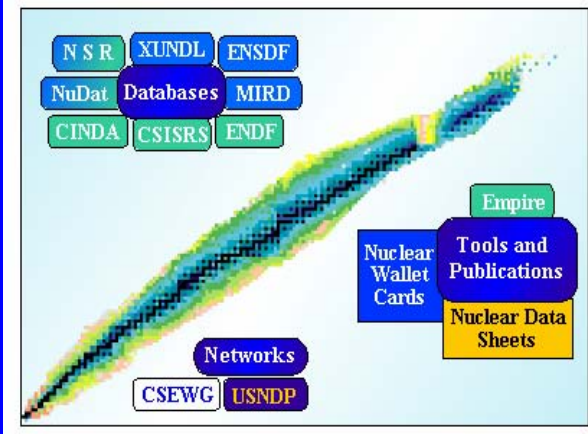

\section{NNDC}

National Nuclear Data Center

BRDOKHAUEN

Nuclear Structure and Decay Databases

Nuclear Structure and Decay Tools

Nuclear Reaction Databases

Nuclear Reaction Tools

Bibliography Databases

Networks and Links

About the Center

Publications

Meetings

- Bibliography databases CINDA, NSR

- Experimental database CSISRS

- Evaluated database ENDF

- Atlas of Neutron Resonances (New)

- Nuclear Reaction Model Code Empire 


\section{Bibliography Databases}

CINDA: Computer Index of Nuclear Reaction Data

- References to 275,000 neutron reactions from 55,000 works $\rightarrow$ comprehensive for neutron reactions

- Charged-particle and photonuclear reactions added in 2005

- In future: CINDA fully derived database, index to CSISRS

NSR: Nuclear Science References

- References describing contents of 180,000 articles from more than 80 journals

- Weekly updated by the National Nuclear Data Center

- Contains also references on neutron reactions

\section{ORELA in bibliography databases}

- All papers duly included

- Databases provide no explicit information on laboratories

\section{Experimental Database CSISRS \\ NNDC BRogkhiven \\ Cross Section Information Storage \& Retrieval System \\ - Also known as EXFOR \\ - Cross-sections from more than 15,500 experiments \\ - Neutron reactions ( 100\%), charged-particles, photonuclear ( 20\%)}
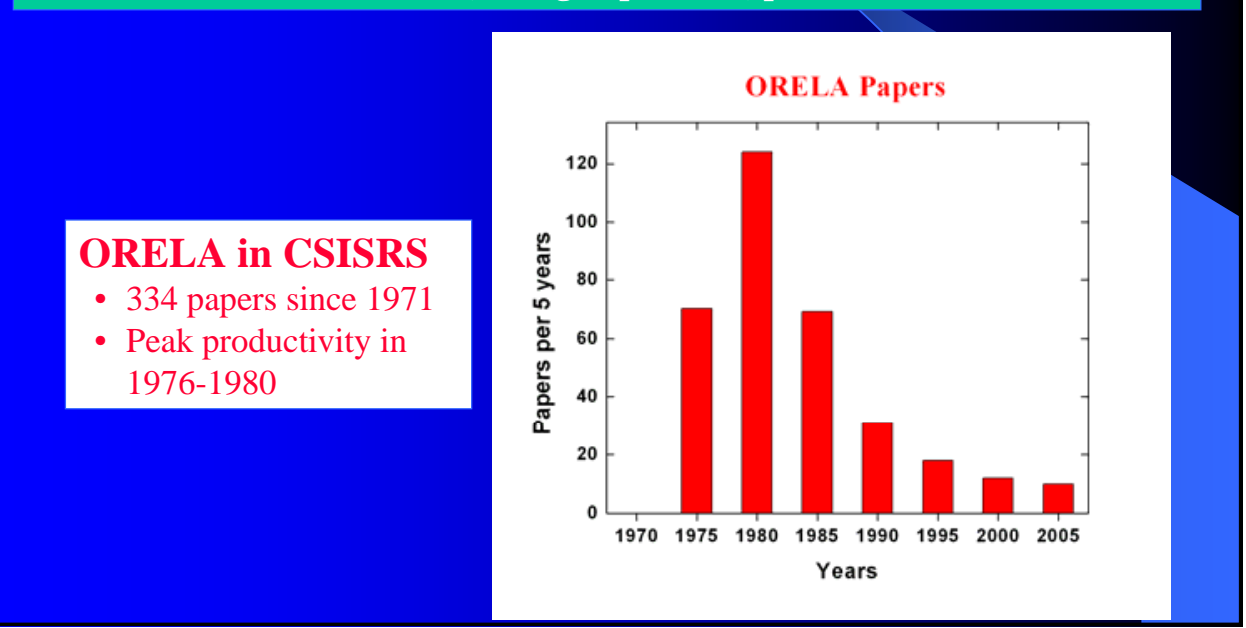

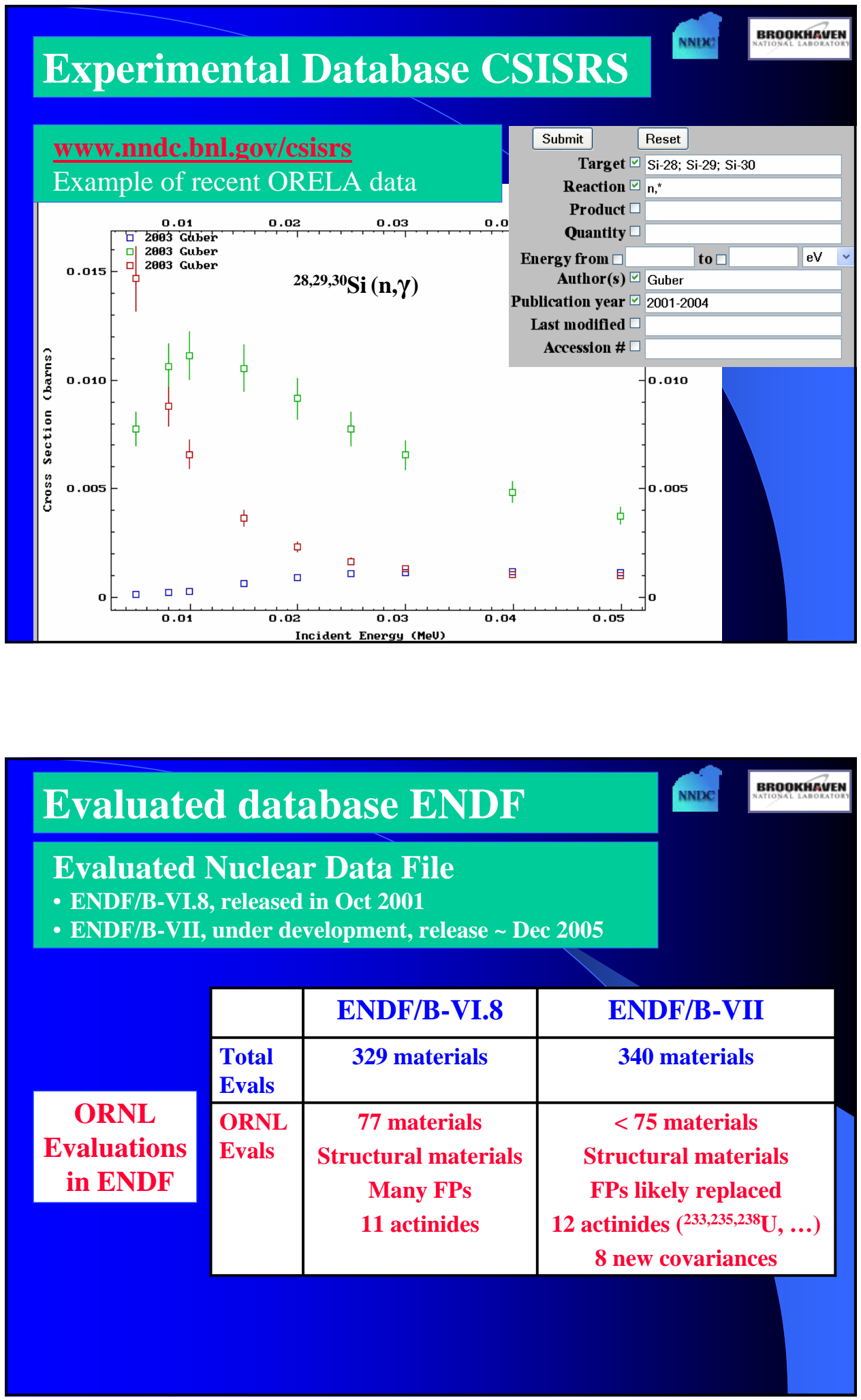


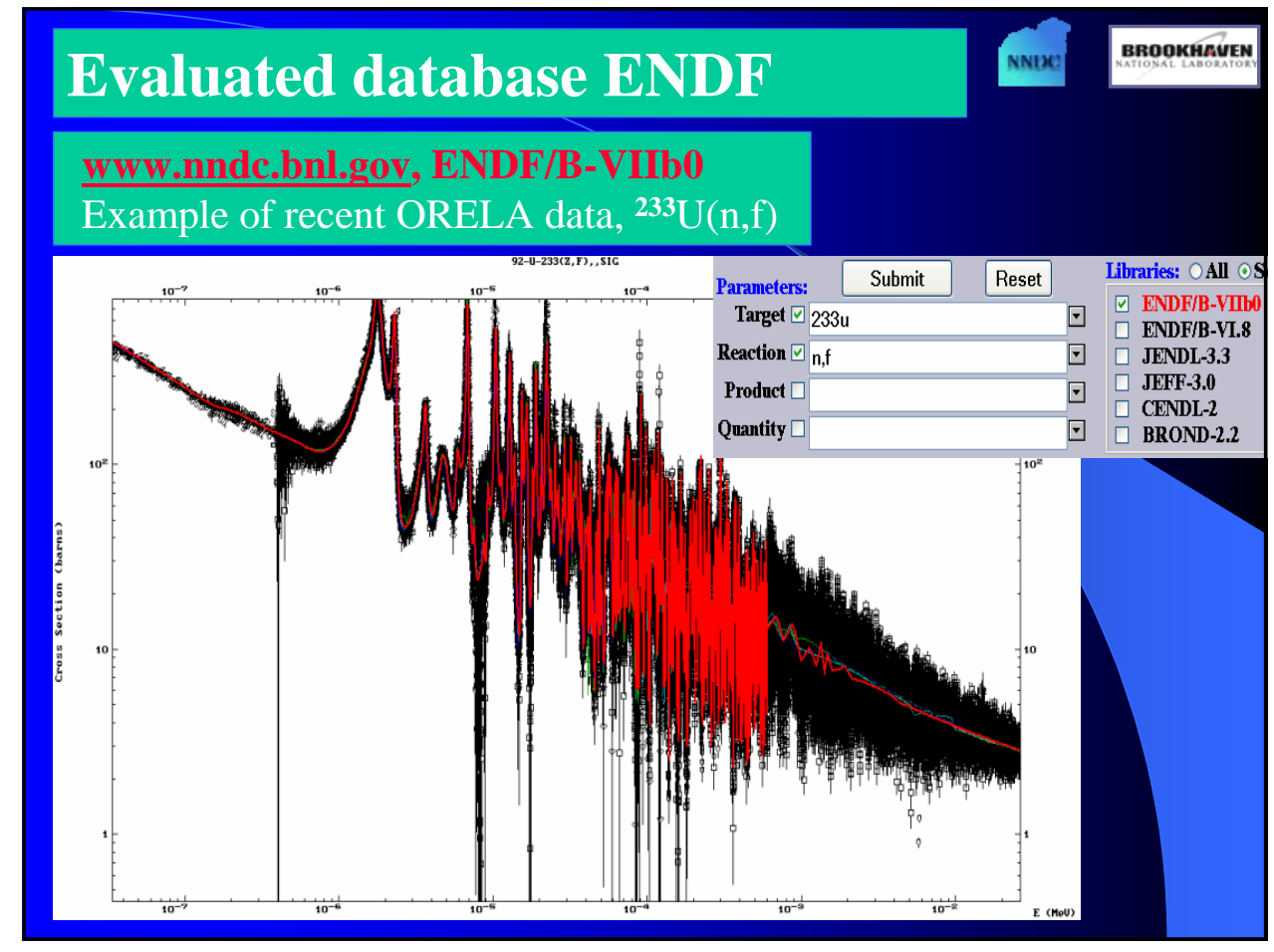

\section{Atlas of Neutron Resonances}

$5^{\text {th }}$ issue of BNL-325 $4^{\text {th }}$ issue in 1981 and 1984

Neutron resonance parameters

Thermal cross-sections

Average resonance parameters

$$
Z=1-100
$$

Submit by August 15, 2005 Publish by Elsevier early 2006

Challenge:

Create ENDF-6 formatted files Create database of $\sigma_{0}$, RI, $D_{0}$ etc
Atlas of Neutron

Resonances

Resonance Parameters and Thermal Cross Sections Part $A: Z=1-60$ S.F. Mughaghab

Atlas of Neutron

$\underline{\text { Resonances }}$

Resonance Parameters and Thermal Cross Sections Part B: Z=61-100 S.F. Mughaghab 


\section{Atlas of Neutron Resonances}

\section{Direct impact of ORELA (data)}

- Large amount of data from ORELA used in Atlas

- Data by Harvey, Macklin, more recently by Koehler, Guber, ...

- Data mostly for

$>$ Structural materials

$>$ Fission products

$>\mathrm{Lu}-\mathrm{Pb}$ region

$>$ Th-232, Pa-231, U-233, U-238, Np-237, Am-241, 242m

$>\mathrm{O}-16, \mathrm{~F}-19, \mathrm{Cl}-35,37$

- But: little attention was given to rare-earth region

\section{Broader impact of ORELA (model calculations)}

- Nuclear level densities ( $\mathrm{D}_{0}$ values)

- Neutron capture ( $\Gamma_{\gamma}$ values)

- Optical model parameterization $\left(\mathrm{S}_{0}, \mathrm{~S}_{1}\right.$ values $)$

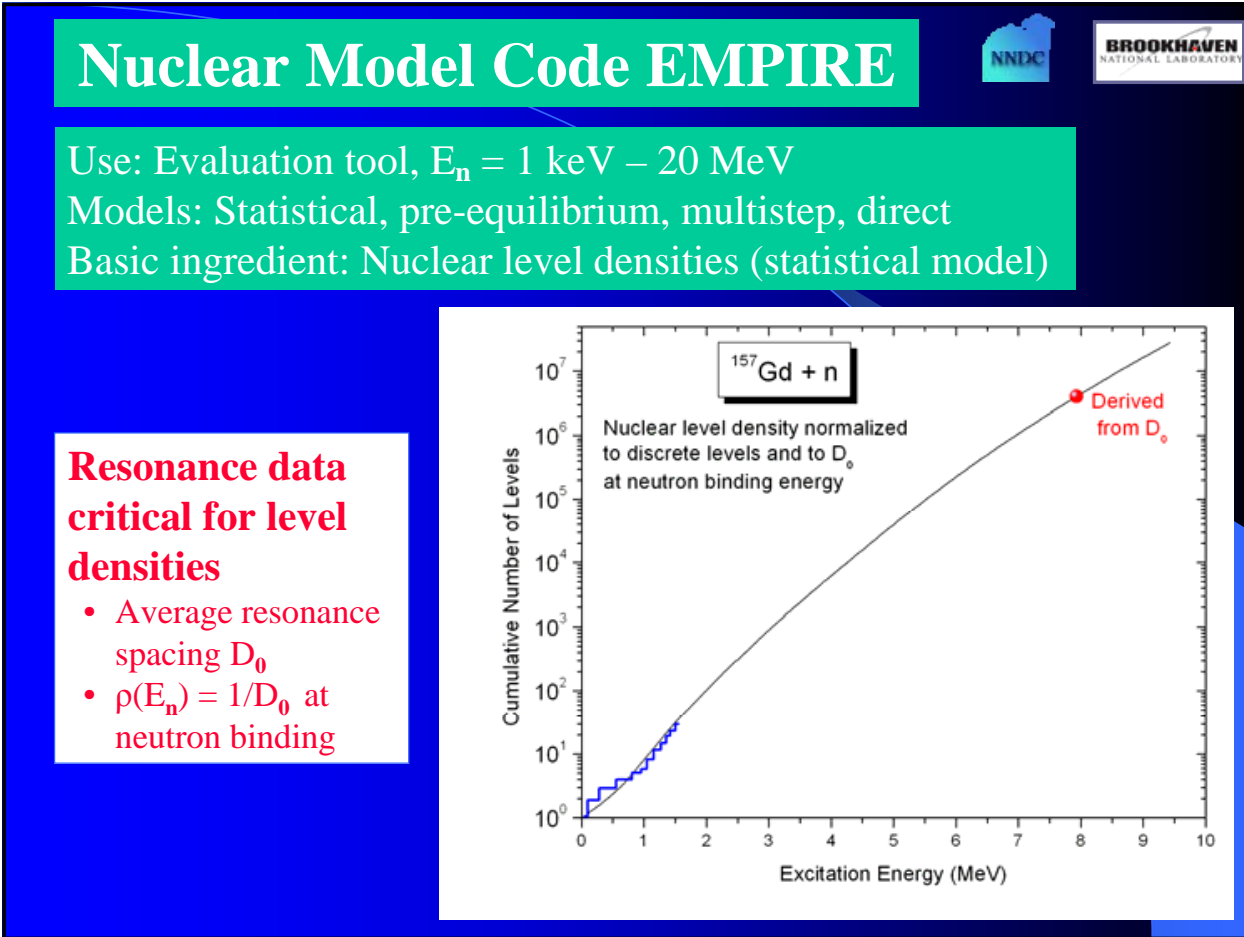




\section{Conclusions}

Impact of ORELA

Profound impact on neutron data and reaction databases

Particular strength - high resolution neutron resonance data Impact on nuclear technology

Impact on nuclear reaction physics

Future of ORELA

Preservation of unique capabilities

User needs (criticality safety, energy, astrophysics, ...)

Funding 


\title{
ORNL Neutron Science Overview
}

\author{
Paul Koehler \\ Physics Division \\ Oak Ridge National Laboratory
}
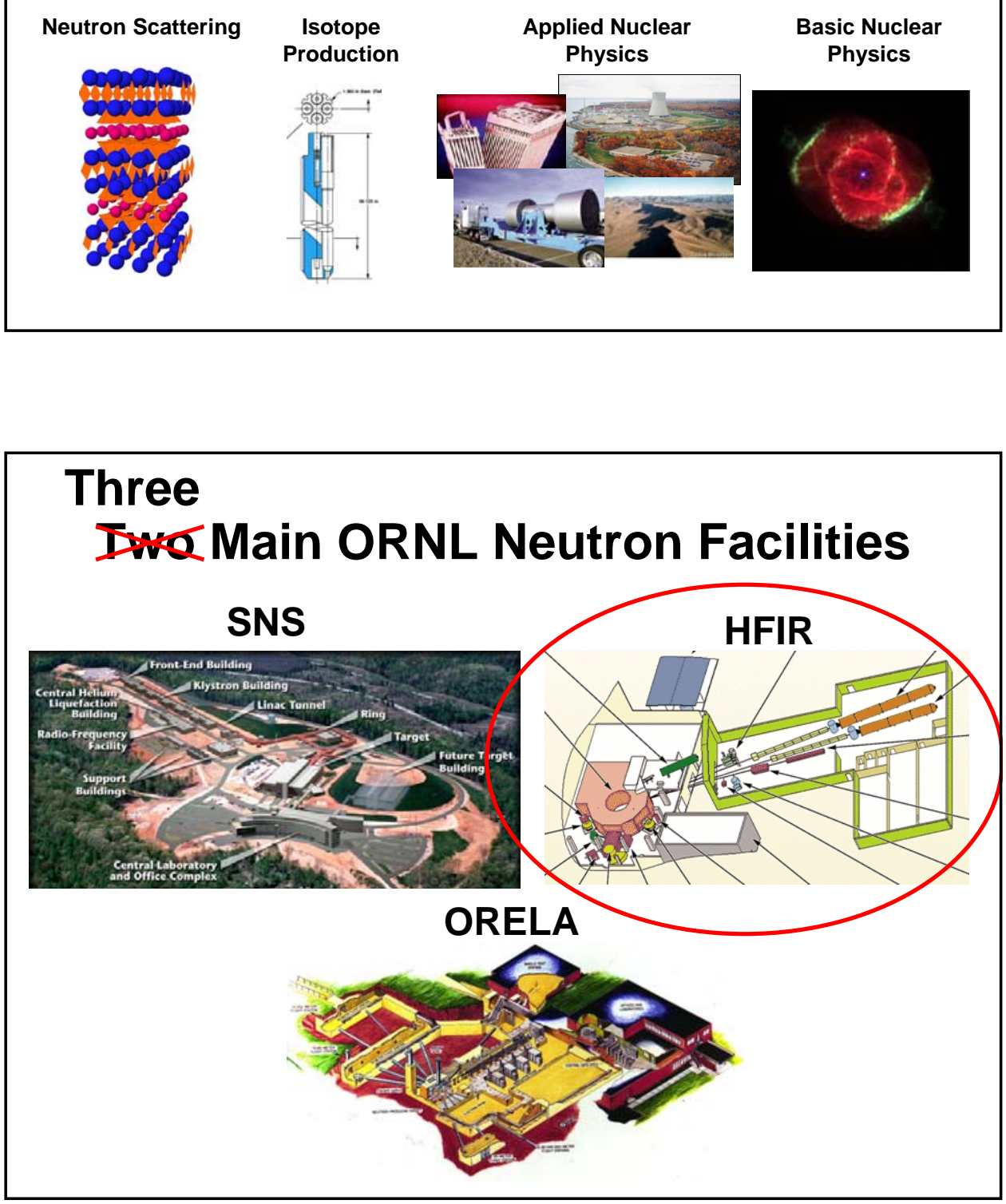


\section{High Flux Isotope Reactor (HFIR)}

- Began full-power operation in 1966.

- $85 \mathrm{MW}$, Be reflected, light water cooled and moderated, flux trap design, HEU fuel.

Fluxes up to $2 \times 10^{15} \mathrm{~cm}^{-2} \mathrm{~s}^{-1}$.

- Four horizontal beam tubes for neutron scattering.

- New cold source and experiment hall.

- Numerous irradiation positions, some of which allow insertion and removal of samples while reactor is operating.
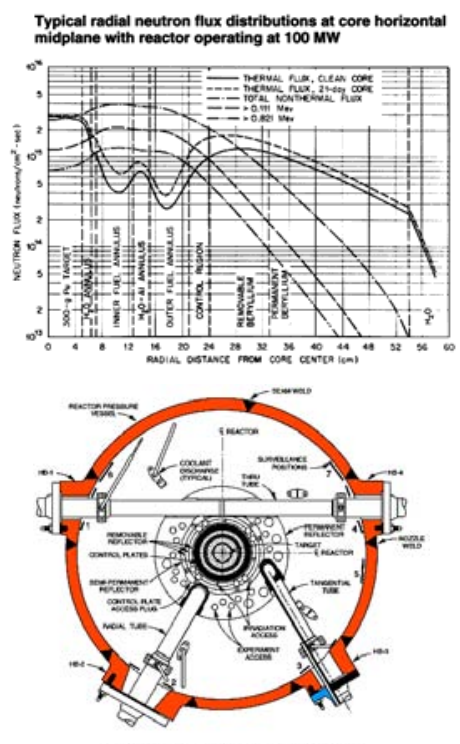

Sectional plan view of reactor core

\section{HFIR Neutron-Scattering Instruments}

- Upgraded HFIR will eventually have 15 instruments. Ones in blue boxes are in the user program.

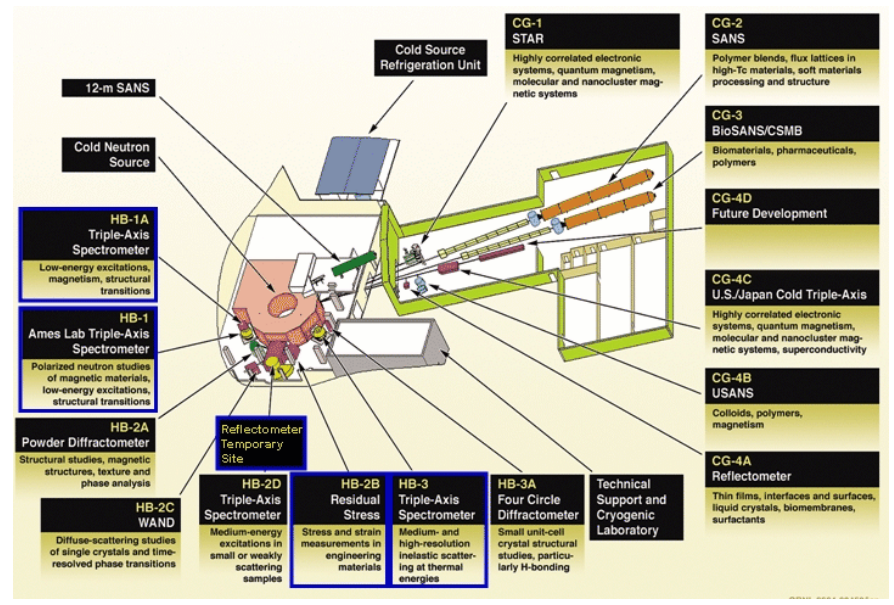




\section{Oak Ridge Electron Linear Accelerator (ORELA)}

- Began operations in 1969.

- 50 kW, electron-linac-driven white neutron source. $\phi=0.8 \times 10^{14} \mathrm{n} / \mathrm{s}$.

- Pulsed source for high-resolution time-of-flight experiments.

- Pulse width ( $\left.\Delta \mathrm{t}_{\mathrm{e}}=2-30 \mathrm{~ns}\right)$ and repetition rate $(1-1000 \mathrm{~Hz})$ variable to suit experiments.

- Nine (11 possible) flight paths from 8 to $200 \mathrm{~m}$.

- Electron, $\gamma$-ray, and neutron irradiations possible.

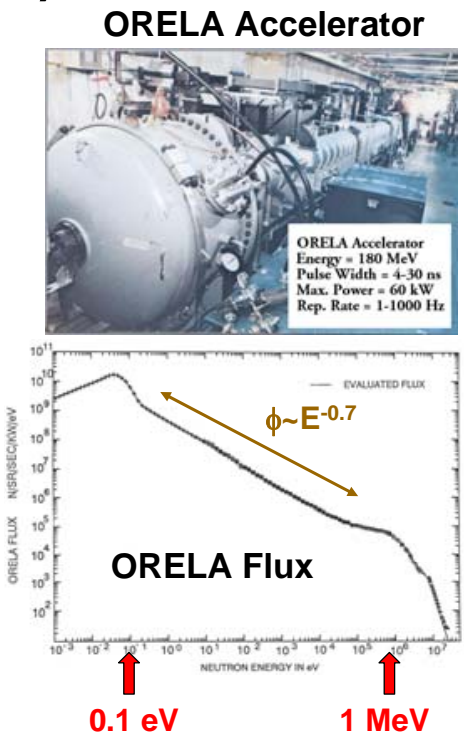

\section{ORELA Neutron Science Instruments}

- Four "standard" instruments for neutron total, $(n, \gamma)$, and $(n, \alpha)$ experiments.

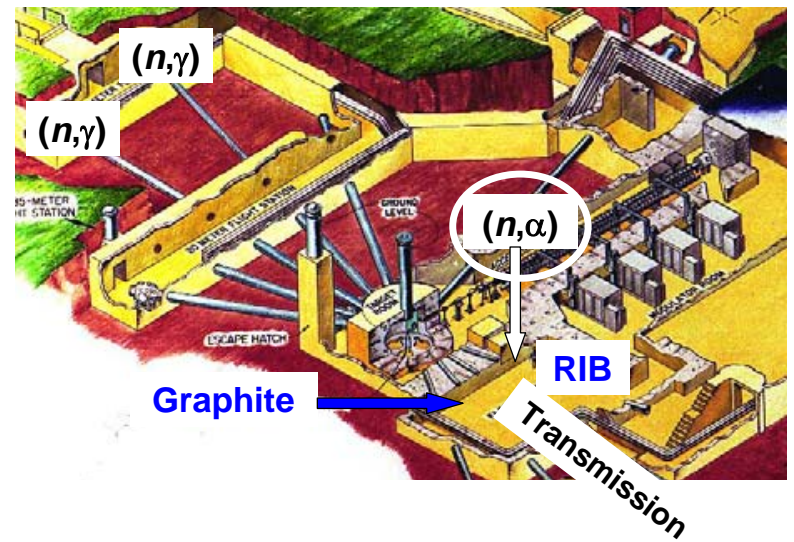

-User experiments for RIB production and graphite moderation. 


\section{$(n, \alpha)$ Measurements at ORELA}

- Compensated Ion Chamber (CIC)

Reduces overload signal due to $\gamma$ flash. Measurements to much higher energies.
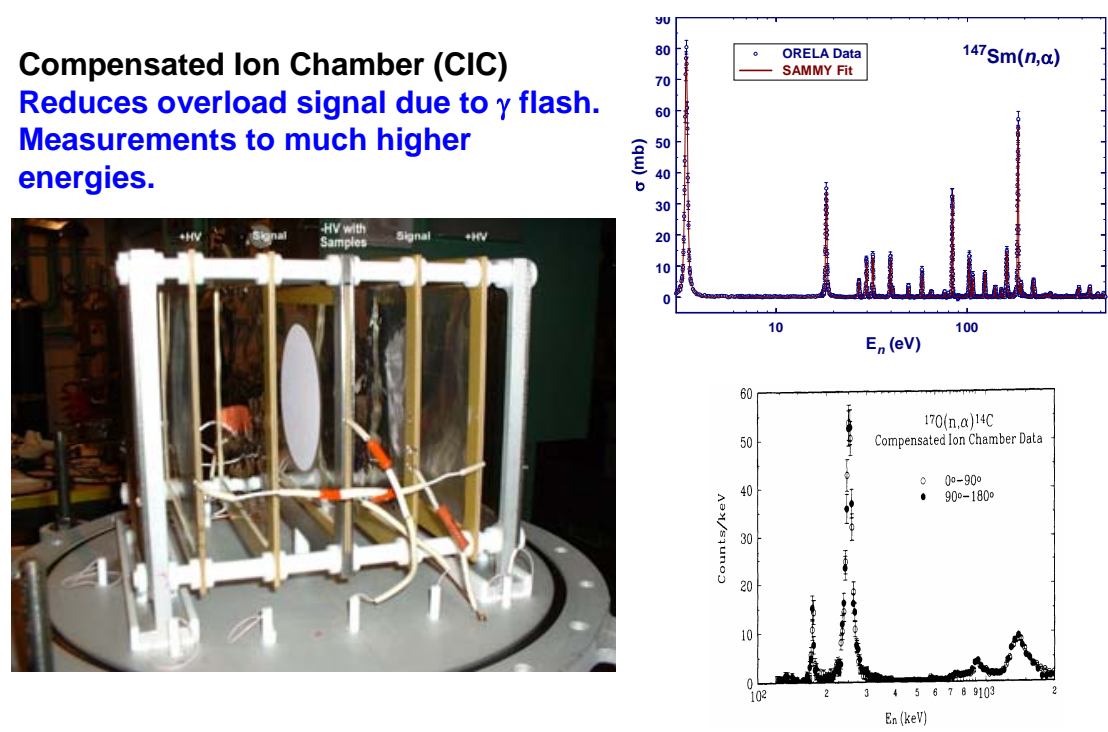

\section{Spallation Neutron Source (SNS)}

- Scheduled for 2006 turn on.

- 1.4 MW, 1-GeV protondriven white neutron source.

- Pulsed source allows moderate resolution timeof-flight experiments $\left(\Delta t_{p}=695\right.$ ns, FWHM 350 ns, $60 \mathrm{~Hz})$.

- 18 flight paths.

- Three cold and one ambient moderators.

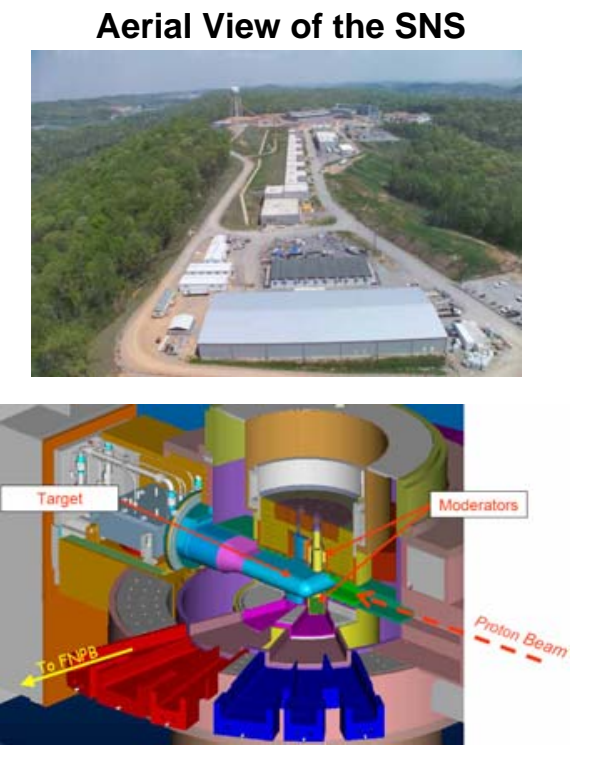




\section{SNS Neutron Science Instruments}

- All instruments but one (fundamental physics) for neutron scattering.

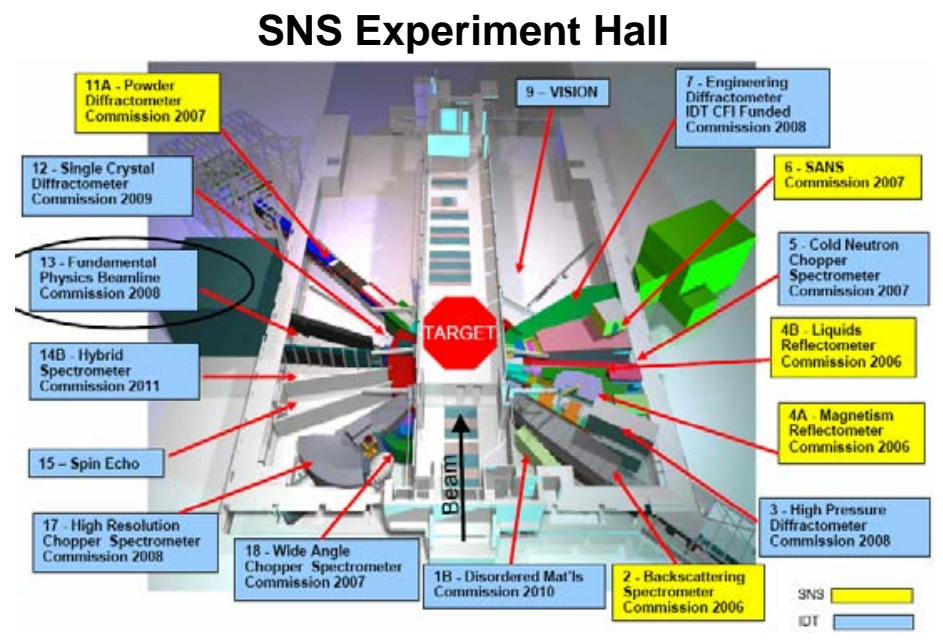

\section{SNS Fundamental Physics Instrument}

- Beam line has two stations; one for CN's, second for UCN's.

- Four choppers to define phase space.

- Monochromator selects 8.9 A neutrons.

- Guides preserve flux and reduce background.

- TOF used to reduce backgrounds and systematic uncertainties.

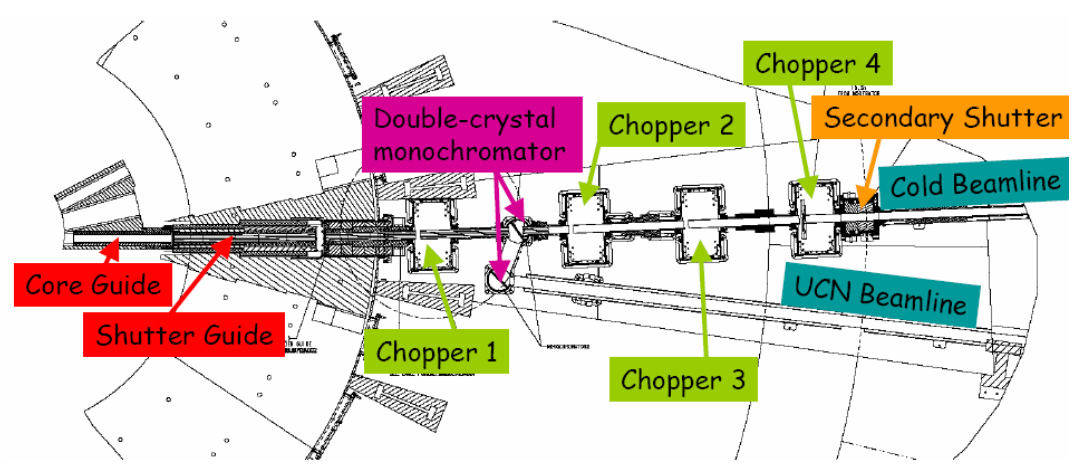




\section{SNS Fundamental Physics Instrument}

- Cold neutron flux.

Red, grey, and blue areas can be selected by choppers to eliminate frame overlap and "flash".

Dips at 8.9/n A due to

monochromator.

Expected mid-2007.

- Ultra-cold neutrons flux.

UCN's made via a superthermal process in superfluid helium. Expected early-2010.

- Highest peak neutron source intensity in the world.

- Time-averaged neutron fluence will be greater than that at any continuous neutron source in the U.S.
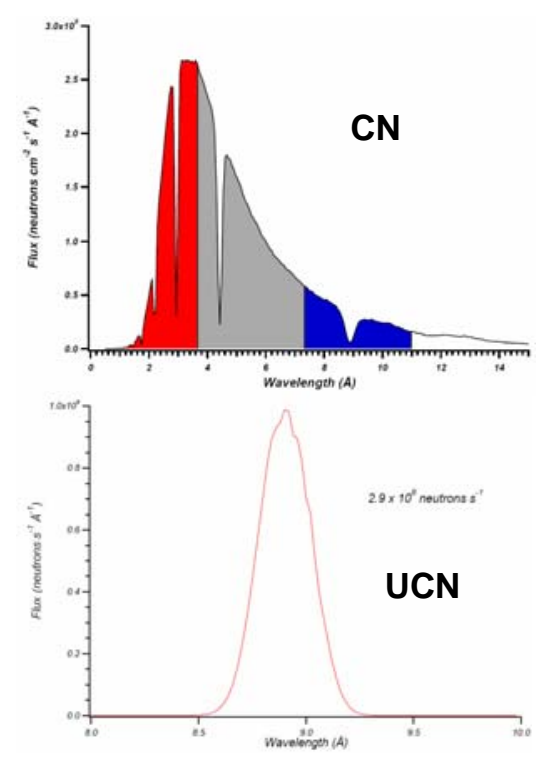

\section{Potential SNS ASAP Instrument}

- Very high flux at the SNS would allow:

Measurements on very small samples for astrophysics and applied physics.

High statistics tests of symmetry (parity and time reversal invariance) violations for basic nuclear physics.

- Workshop held in March 2002 to explore the possibilities.

- Would be complementary to ORELA capabilities.

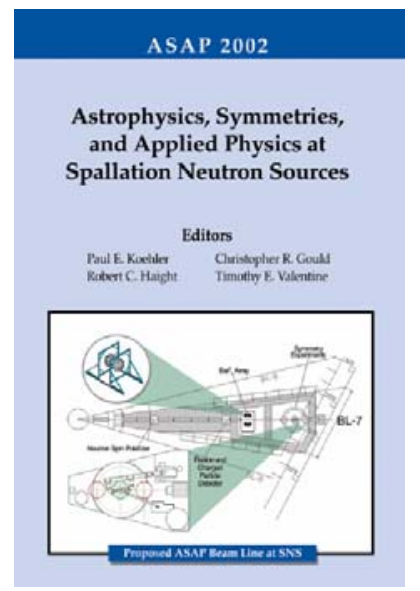




\section{Complementarity of ORELA and SNS}

- Moore's Law:

You can learn more and more about less and less

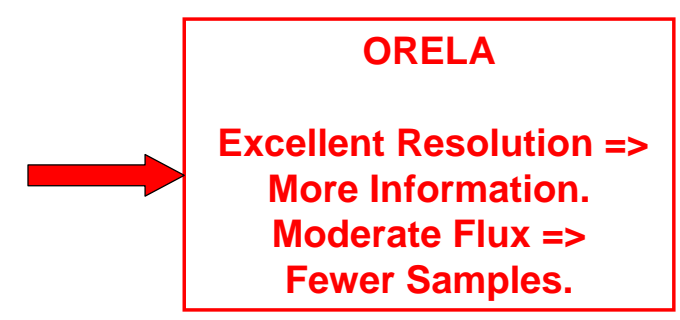

or

You can learn less and less about more and more.

SNS

Moderate Resolution =>

Less Information.

Very High Flux => More Samples.

\section{Types of (Experimental) Neutron Science at ORNL}

- Neutron Scattering. Chemistry Complex Fluids Crystalline and Disordered Materials Engineering Magnetism and Superconductivity Polymers Structural Biology
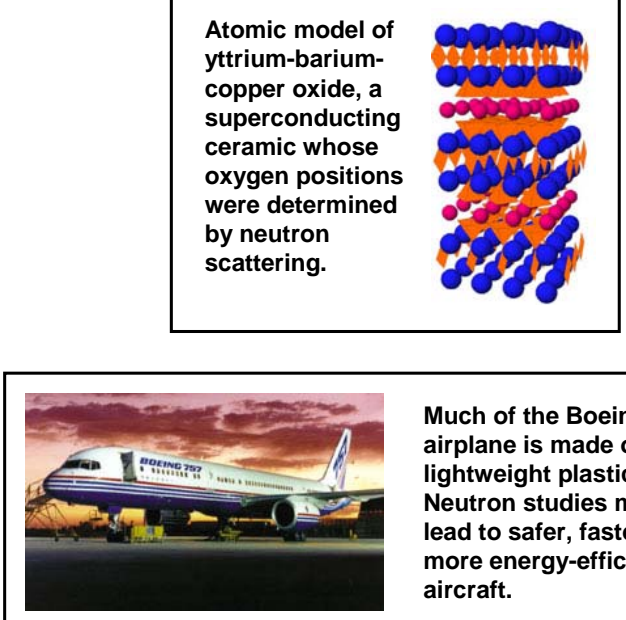

Much of the Boeing 757 airplane is made of lightweight plastic. Neutron studies may lead to safer, faster, more energy-efficient aircraft. 


\section{Neutron Science at ORNL}

- Isotope production.

${ }^{252} \mathrm{Cf}$ - several medical and industrial applications. HFIR is the West's sole source.

${ }^{43} \mathrm{~K}$ - evaluation of coronary heart disease.

${ }^{103} \mathrm{Pd}$ - treatment of prostate cancer.

${ }^{153} \mathrm{Gd}$ - for measuring bone loss.

${ }^{188} \mathrm{~W}$ - associated with treatment of cancer and arthritis.

- Activation analysis and materials irradiation.

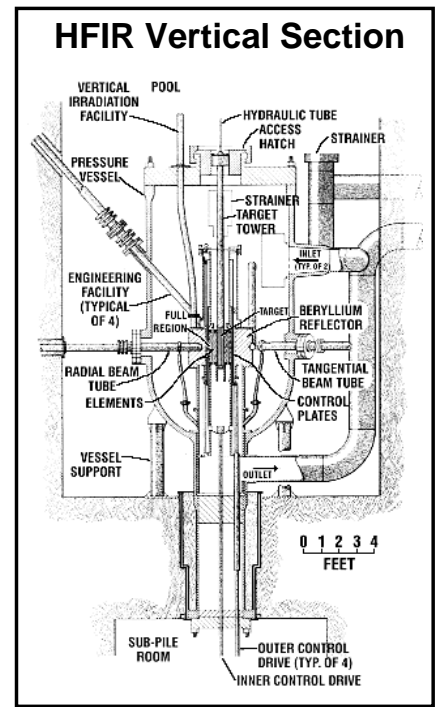

\section{Neutron Science at ORNL}

- Fundamental physics.

Neutron beta decay as:

(1) A detailed probe of the nature of the electroweak theory.

(2) A test of the unitarity of the Cabibbo-Kobayashi-Moskawa (CKM) matrix.

(3) A probe of physics beyond the Standard Model (SM).

(4) An important input to the theory of Big Bang Nucleosynthesis.

The study of the nature of the weak interaction between hadrons.

Via the measurement of parity non-conserving (PNC) effects in simple two-particle systems such as n-p, n-d, and $n-\alpha$.

The study of the nature of time reversal non-invariance and the origin of the cosmological baryon asymmetry.

Through a search for a non-zero neutron electric dipole moment. 


\section{Neutron Science at ORNL}

- Nuclear Astrophysics and Basic Nuclear Physics. Many new $(n, \gamma)$, neutron total, $(n, \alpha)$, and $\left(n, n^{\prime}\right)$ measurements are needed to:

Test and improve models of nucleosynthesis in stars and supernovae, and of galactic chemical evolution.

Estimate the age of the universe.

Understand the origin of meteoric isotopic anomalies. Improve nuclear models.

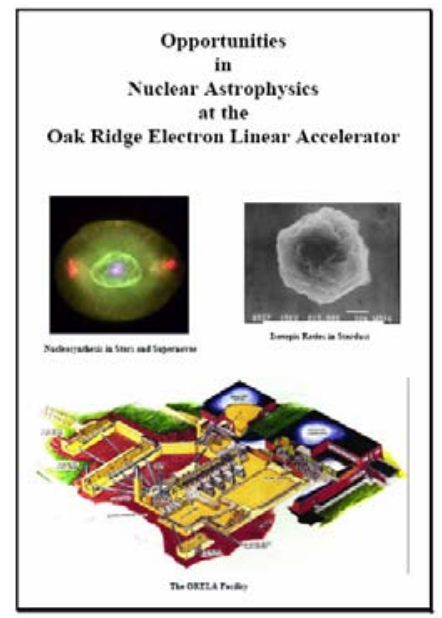

\section{Neutron Science at ORNL}

- Applied Nuclear Physics.

Many new $(n, \gamma)$, neutron total, $(n, f)$, and $\left(n, n^{\prime}\right)$ measurements are needed for:

Criticality safety.

Generation IV reactors.

Advanced Fuel Concepts Initiative.

Transmutation.

- Many of these measurements could be made at ORELA (and the SNS).
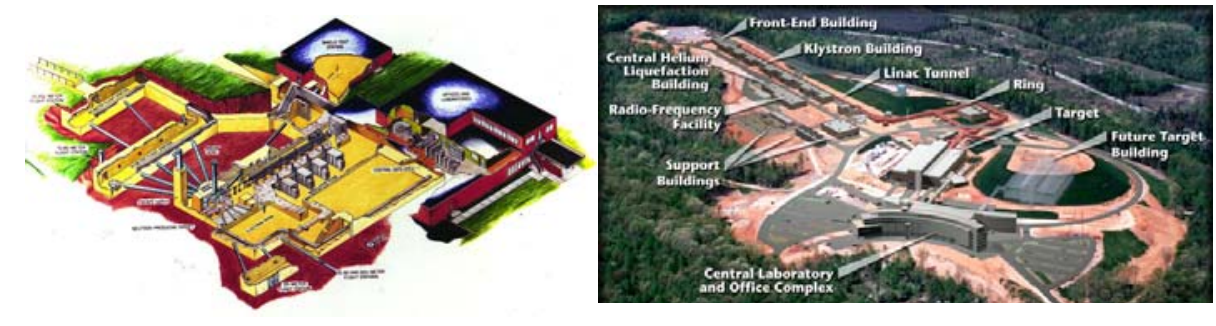



\section{Neutron Cross Section Me asurements at ORELA}

K. H. Guber ${ }^{1}$, L. C. Leal $l^{1}$, g.A. Harvey ${ }^{2}$, R. O. Sayer $r^{1}, \mathcal{H}$. Derrien ${ }^{1}, \mathcal{D}$. A. Wiarda and P. E. Koefler ${ }^{2}$

${ }^{1} \mathcal{N u c l e a r}$ Science and Technology Division ${ }^{2}$ Physics Division

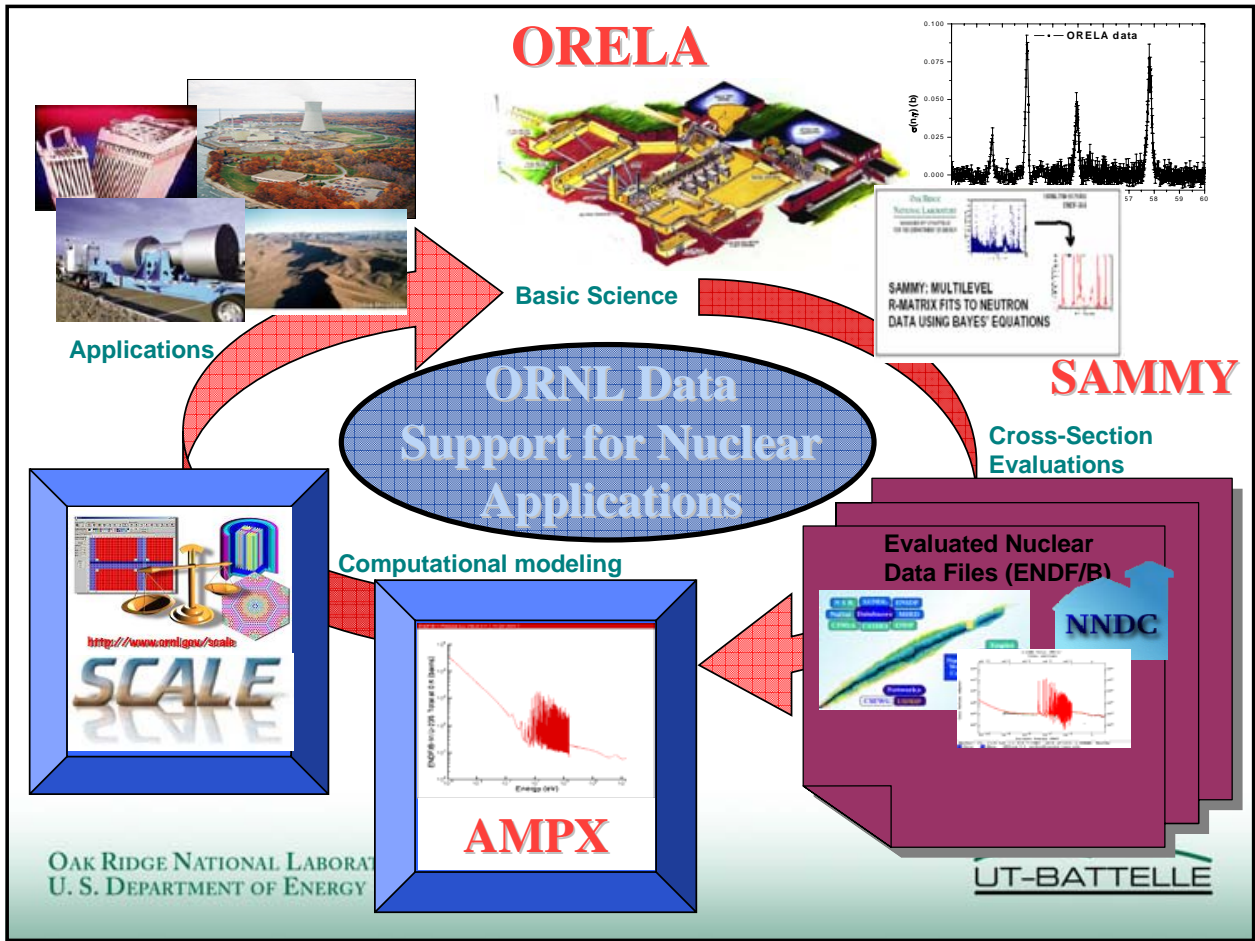




\section{$O R E \perp A$}

- Unique user facility remaining in U.S.

- High flux $\left(10^{14} \mathrm{n} / \mathrm{sec}\right)=>$ gram. sized, affordable samples

- Excellent resolution $(\Delta t=2-30 n s)$ $=>$ good $S / \mathcal{N}$ facilitates better e valuations

- "White" neutron spectrum from $\mathcal{E}_{n} \sim 0.01 \mathrm{eV}-80 \mathcal{M e} \mathcal{V}=>$ reduces systematic uncertainties

- Measurement systems well understood =>very accurate data

- Simultaneous measurements => $(n, \gamma),(n, \alpha),\left(n, n^{\prime}\right)$, and $\sigma_{\text {total }}$ experiments at the same time on different beam lines

- Measurements on over 180 Isotopes
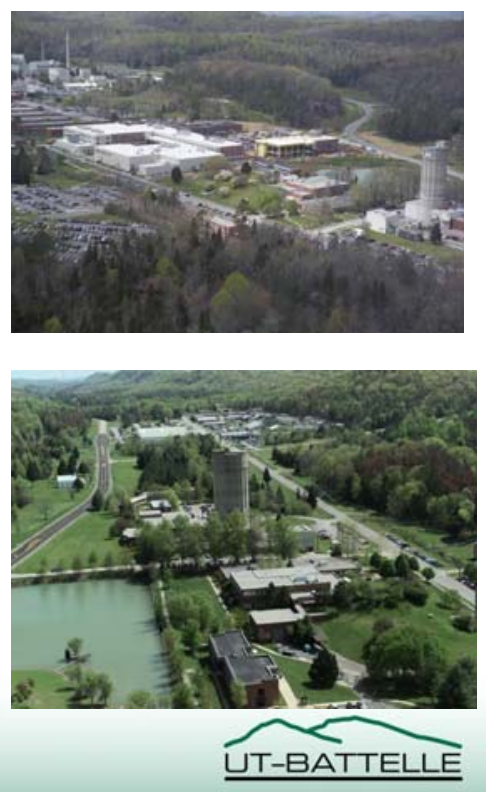

\section{Existing Experiments at ORELA}

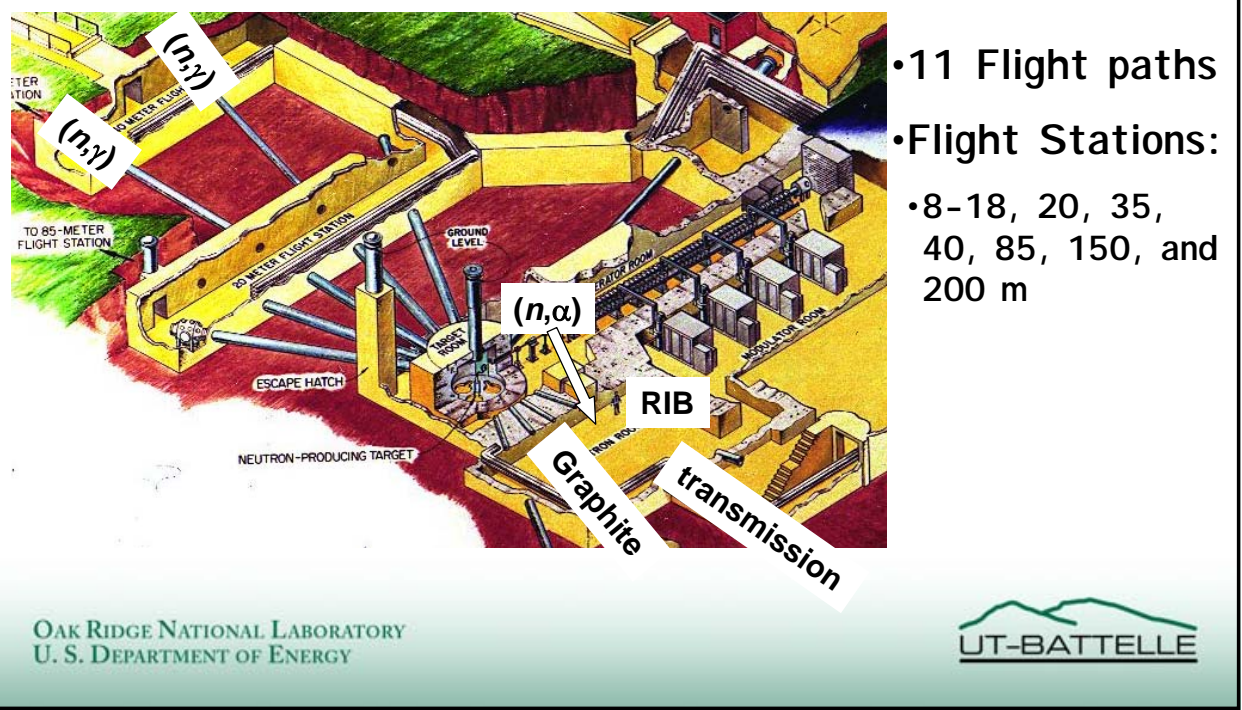




\begin{tabular}{|c|c|c|c|c|c|c|}
\hline $\mathrm{Crc}$ & $S-\mathcal{S}$ & $t i o n$ & Me asu & $m e n t$ & $\mathcal{F a c}$ il & ies \\
\hline \multirow{2}{*}{$\begin{array}{c}\text { Facility } \\
\text { Parameters }\end{array}$} & \multicolumn{4}{|c|}{ United States } & \multicolumn{2}{|c|}{ Europe } \\
\hline & ORELA & LANSCE & IPNS & RPI & GELINA & n_TOF \\
\hline Source & e' linac & p spallation & p spallation & e- linac & e- linac & p spallation \\
\hline \begin{tabular}{|l}
$\begin{array}{l}\text { Particle E } \\
\text { (MeV) }\end{array}$ \\
\end{tabular} & 140 & 800 & 450 & $>60$ & 120 & 20000 \\
\hline Flight Path (m) & $10-200$ & $7-55$ & $\sim 6-20$ & $10-250$ & $8-400$ & 185 \\
\hline $\begin{array}{l}\text { Pulse Width } \\
\text { (ns) }\end{array}$ & $2-30$ & 125 & 40 & $15-5000$ & $1-2000$ & 7 \\
\hline $\begin{array}{l}\text { Max Power } \\
\text { (kW) }\end{array}$ & 50 & 64 & 6.3 & $>10$ & 11 & 45 \\
\hline Rep Rate (Hz) & $1-1000$ & 20 & 30 & $1-500$ & Up to 900 & $0.278-0.42$ \\
\hline \begin{tabular}{|l|} 
Best Intrinsic \\
Resolution \\
(ns/m)
\end{tabular} & 0.01 & 3.9 & 2.0 & 0.06 & 0.0025 & 0.034 \\
\hline Neutrons/s & $1 \times 10^{14}$ & $7.5 \times 10^{15}$ & $8.1 \times 10^{14}$ & $4 \times 10^{13}$ & $3.2 \times 10^{13}$ & $8.1 \times 10^{14}$ \\
\hline \multicolumn{7}{|c|}{$\begin{array}{l}\text { OAK RIDGE National. Laboratory } \\
\text { U. S. DEPARTMENT OF ENERGY }\end{array}$} \\
\hline
\end{tabular}

\section{Comparison: ORELA, LANSCE, IPNS}

- Each facility has its own characteristics:

-ORELA has very good ne utron energy resolution 6 ut the penalty will be a lower ne utron flux.

- LANS CE and IPNS have higher n-flux per 6urst 6 ut inferior resolution.

$-O R E L A$ is an undermoderated n-source whereas LANS CE and I PNNS are highly moderated sources. This can have severe consequences for the resolution of resonances.

- For comparison usually a figure of me rit (FOM) is used:

$$
n \cdot f\left(u x /(d \mathcal{E} / \mathcal{E})^{2}\right.
$$


Comparison of Existing Experiments

\begin{tabular}{|c|c|c|c|c|c|c|c|c|}
\hline & ORELA & ORELA & ORELA & GELINA & GELINA & $\begin{array}{c}\text { DANCE } \\
\text { at } \\
\text { LANSCE }\end{array}$ & IPNS & n_TOF \\
\hline Flight path length [m] & 200 & 40 & 10 & 400 & 55 & 20 & 20 & 185 \\
\hline Pulse width [ns] & 6 & 8 & 24 & 1 & 1 & 125 & 40 & 7 \\
\hline Power [kW] & 13 & 8 & 50 & 11 & 11 & 64 & 6.3 & 45 \\
\hline Repetition Rate [Hz] & 800 & 525 & 1000 & 800 & 800 & 20 & 30 & 0.42 \\
\hline $\begin{array}{c}\text { Neutron Flux @ 1keV } \\
\text { [neutron/s/cm2] }\end{array}$ & $6.6 \mathrm{E}+01$ & $1.0 \mathrm{E}+03$ & $1.0 \mathrm{E}+05$ & $2.5 E+01$ & $1.3 E+03$ & $1.2 \mathrm{E}+05$ & $1.0 \mathrm{E}+05$ & $2.9 \mathrm{E}+03$ \\
\hline $\begin{array}{c}\text { Intrinsic Resolution } \\
{[\mathrm{ns} / \mathrm{m}]}\end{array}$ & 0.0300 & 0.2000 & 2.4000 & 0.0025 & 0.0182 & 6.2500 & 2.0000 & 0.0378 \\
\hline $\begin{array}{l}\text { Realistic Resolution } \\
\text { dE }[\mathrm{eV}] @ 1 \mathrm{keV}\end{array}$ & 0.08 & 0.38 & 1.94 & 0.05 & 0.36 & 3.48 & 2.5 & 0.49 \\
\hline $\begin{array}{c}\text { FOM @ 1keV } \\
\text { (Flux/(dE/E)^2) } \\
{[\mathrm{n} / \mathrm{s} / \mathrm{cm} 2]}\end{array}$ & $1.16 \mathrm{E}+10$ & $6.85 E+09$ & $2.67 E+10$ & $2.60 \mathrm{E}+10$ & $2.60 \mathrm{E}+10$ & $9.93 \mathrm{E}+09$ & $6.1 \mathrm{E}+09$ & $1.2 \mathrm{E}+10$ \\
\hline
\end{tabular}

OAK Ridge National. Laboratory

U.S. DEPARTMENT OF ENERGY

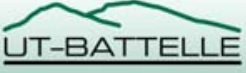

Flux Comparison: ORELA, LANS CE, IPNS

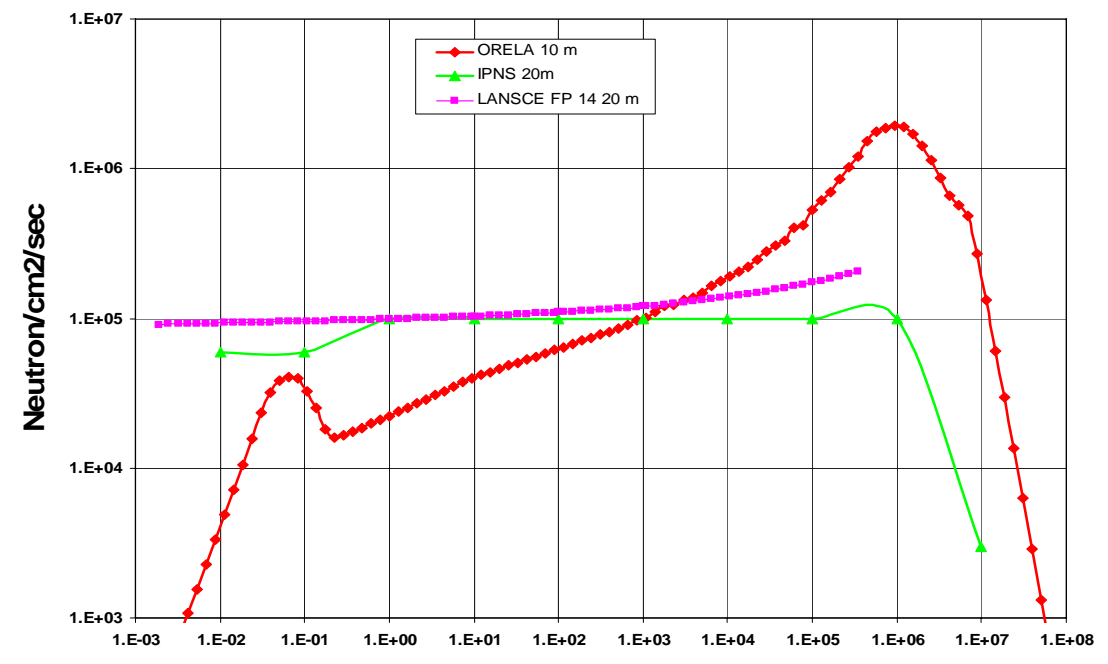

Energy [eV]

OAK Ridge National. Laboratory

U. S. DEPARTMENT OF ENERGY

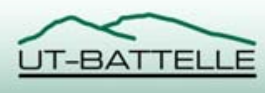




\section{FOM : ORELA, LANSCE, IPNS}

Figure of Merit : Flux/(dE/E)^2

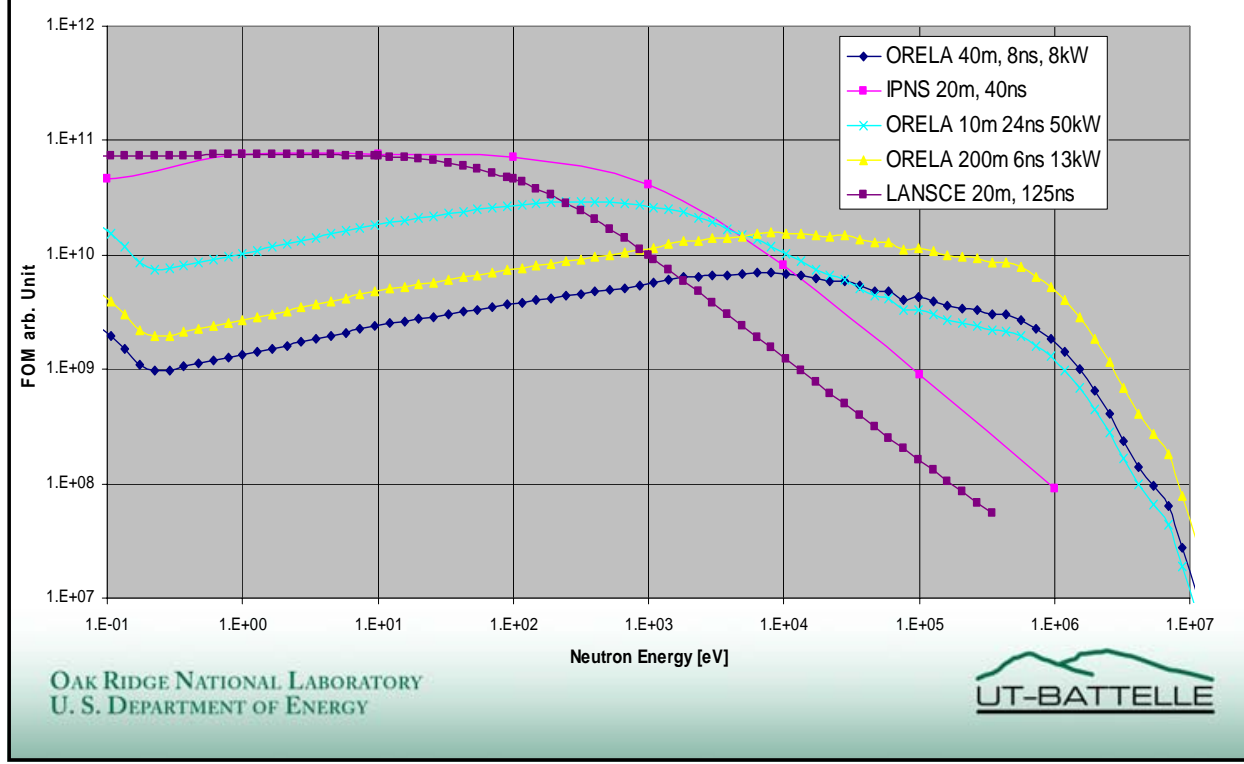

\section{Neutron Cross-Section Measurements In The Resolved Resonance Range}

- Neutron flux is important 6ut alone cannot guarantee accurate measurements.

- Neutron Energy resolution is important.

- Goal to resolve many resonances in order to ob tain reliable average resonance parameters. These are important to perform the analys is of the unresolved energy range and statistical model calculations.

- Re solved resonances help to identify and disentangle is otopic impurities in the sample.

- Re solved resonances help to apply individual and no average correction to the data (self-shielding, multiple. scattering). 


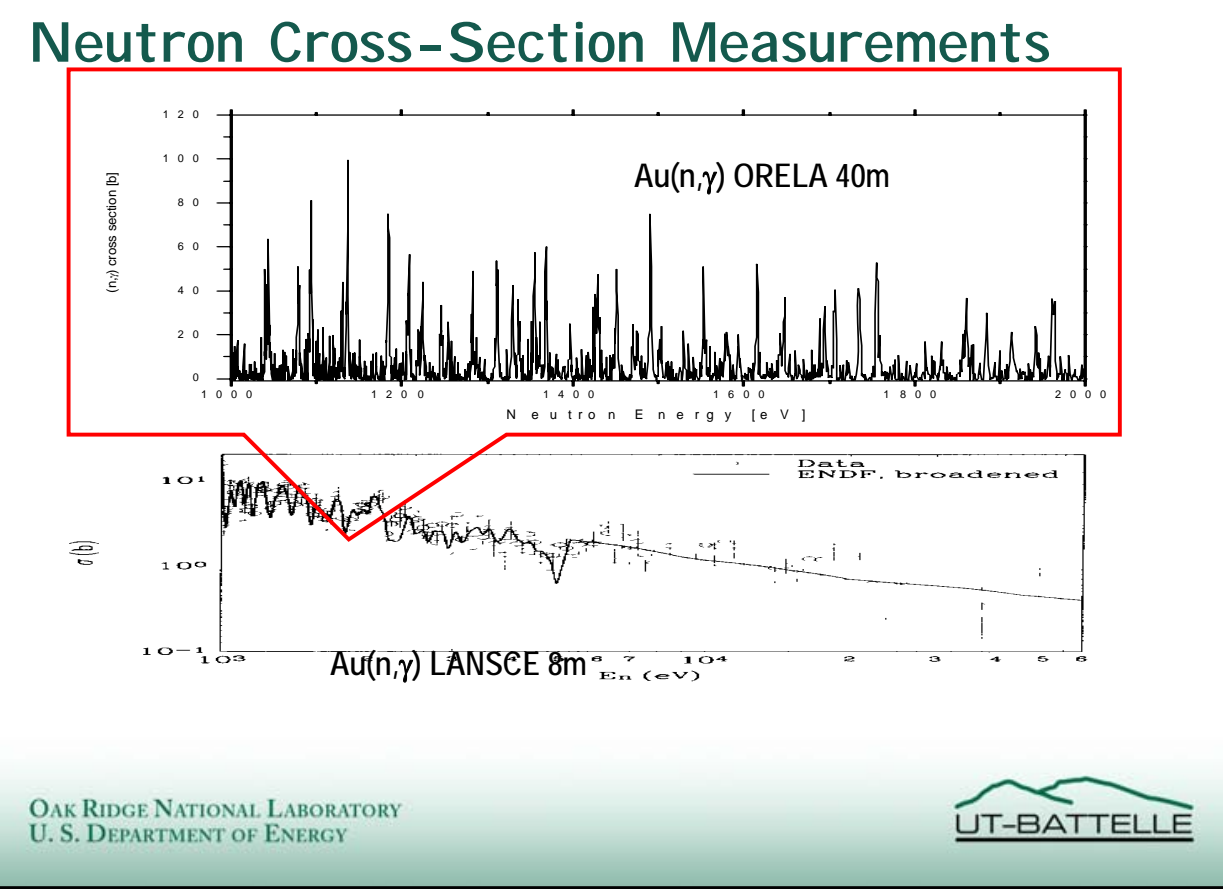

\section{Neutron Energy Resolution}

- Flight path length, the longer the better.

- Pulse width of the neutron burst, the shorter the better:

- Typically fixed with spallation sources (tens of ns to fundreds of ns)

- Linac sources can vary pulse width (1ns up to tens of ns).

- Source moderation distance

- The uncertainty of the creation location of the neutron inside the moderator has to be taken into account for the resolution function.

- ORELA is an undermoderated source with relatively small neutron production target. Spallation sources are usually optimized for thermal neutron flux, this requires large moderators.

- The moderation effect put tails on resonances due to delayed ne utrons.

- Hinders resolution of closely spaced resonances.

- Produces background in unresolved energy range which cannot be corrected. This effect is of the order of $16 \%$ for 20 keV (Coceva et al. 2002) for $n_{-} \mathcal{T} O \mathcal{F}$ and can not be estimated quantitatively. 


\section{Neutron Energy Resolution}

$\mathrm{Au}(\mathrm{n}, \gamma)$ LANSCE $8 \mathrm{~m}$

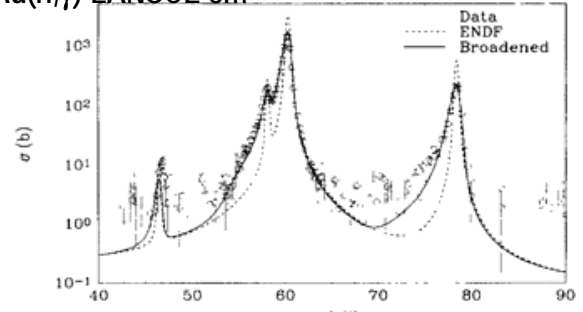

$\operatorname{Au}(\mathrm{n}, \gamma) \mathrm{n} \_$TOF CERN $185 \mathrm{~m}$

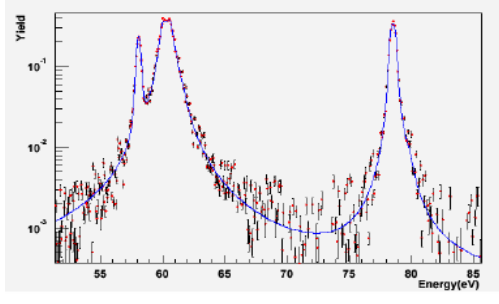

OAK Ridge National. Laboratory

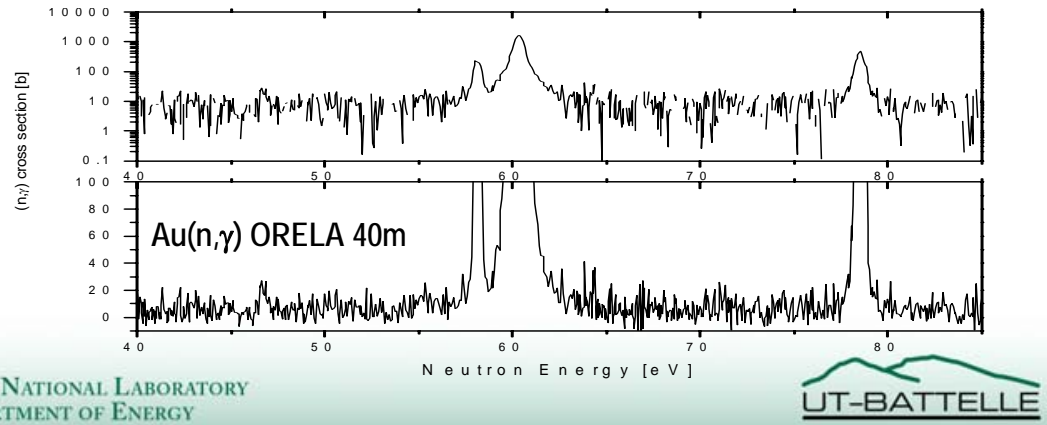

Doppler - Resolution Broadening @20 m for 242Pu

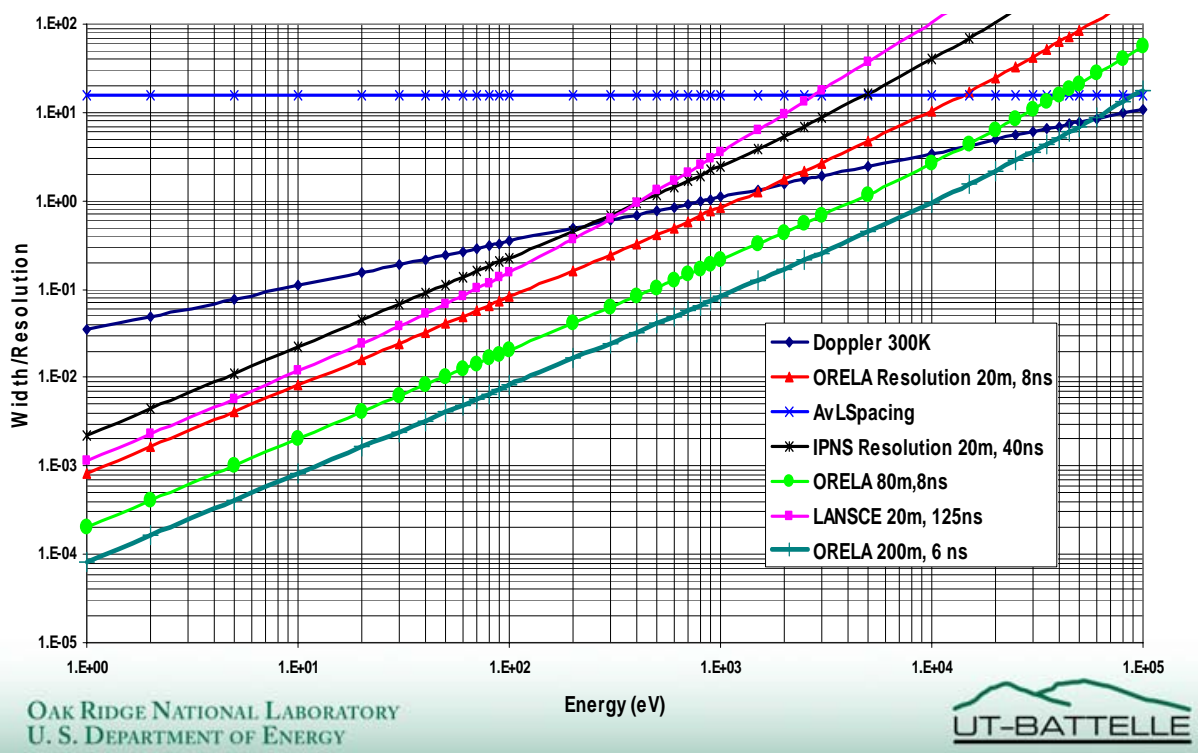


$S \mathcal{A M M}$ fit of the effective total cross and capture cross section of $238 \mathrm{Cl}$ at high $\mathcal{N}($ utron Energies

Energy Range 0 to 20 KeV: 932 s-wave resonances $g^{\pi}=1 / 2+$

$814 p$-wave resonances $g \pi=1 / 2$.

1540 -wave resonances $g \pi=3 / 2$.

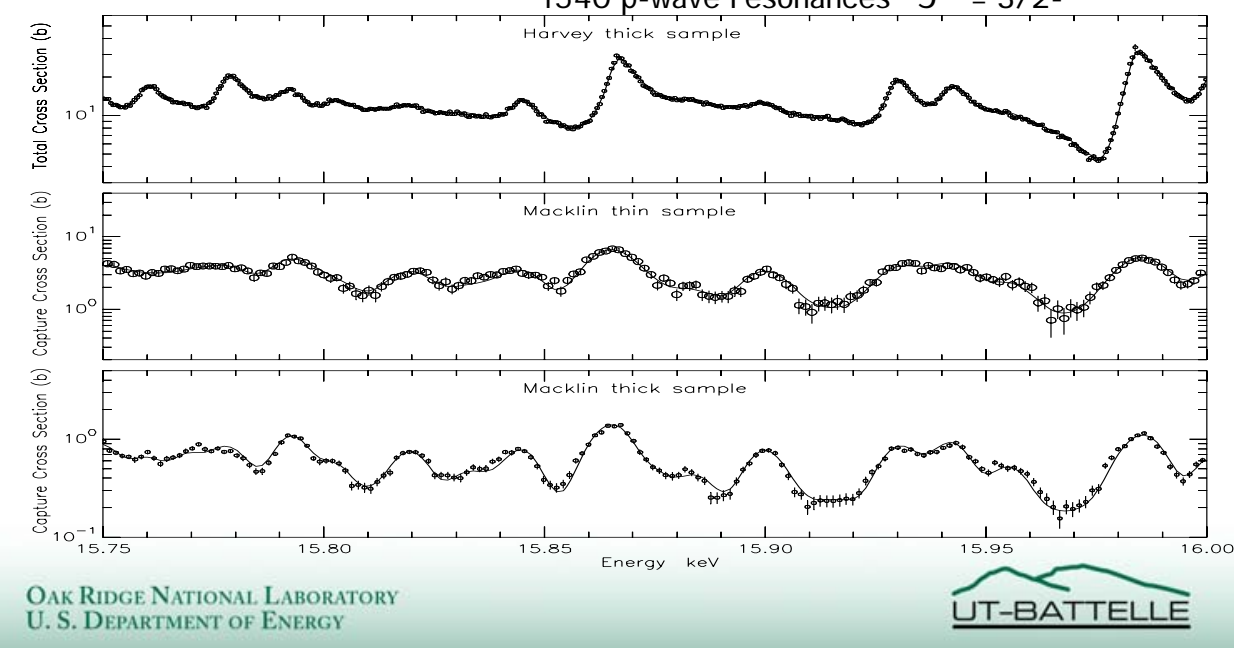

Number of Resonances Versus Neutron Energy

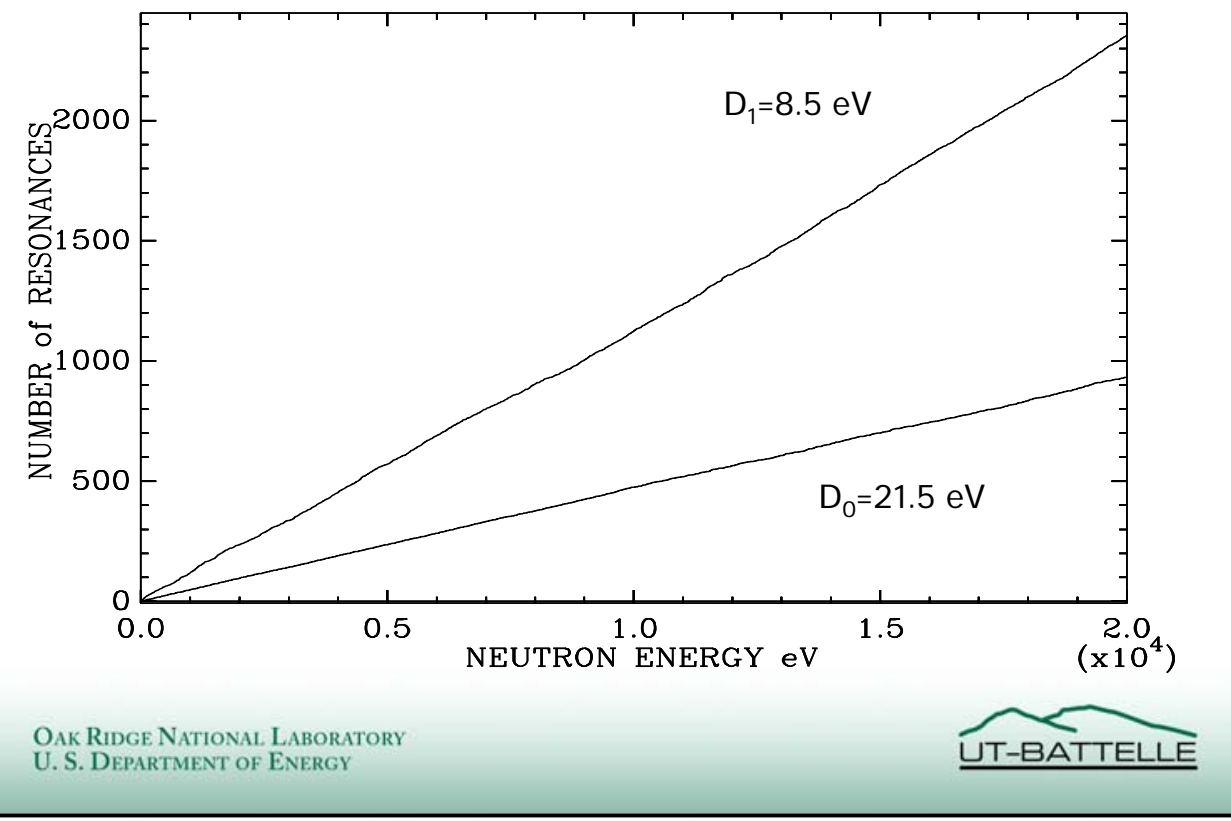




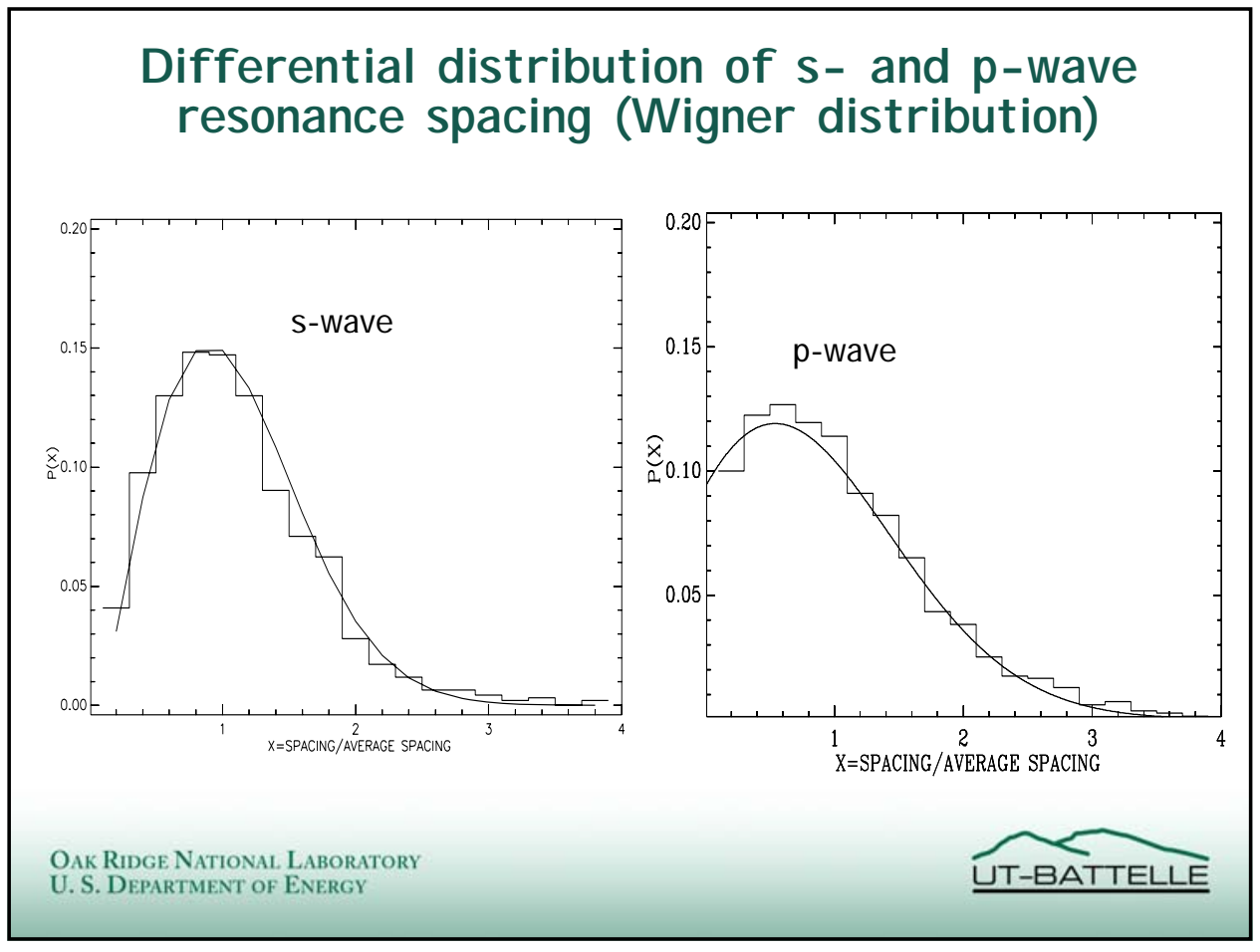

Distributions of The Reduced Neutron Widths (Porter-Thomas distribution)
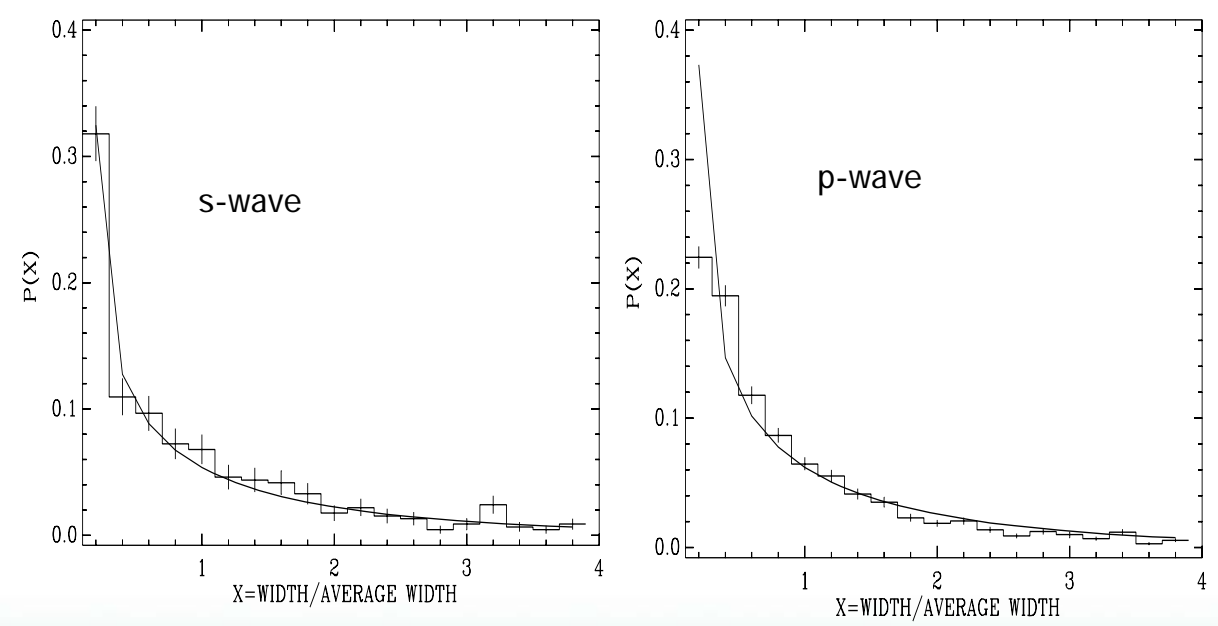

OAK Ridge National. LaBoratory

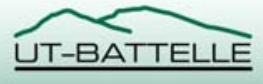


Much of the Old Neutron Data (on Which Current

Evaluations Are Based) Are Seriously Incorrect

- Some problems with the old data:

- Underestimated neutron sensitivity correction

- Low-energy cut off of 3 keV

- No figh energy (>100 keV) data

- Incorrect weighting function

- Poor resolution

- Poorly characterized samples, i.e. water in the sample

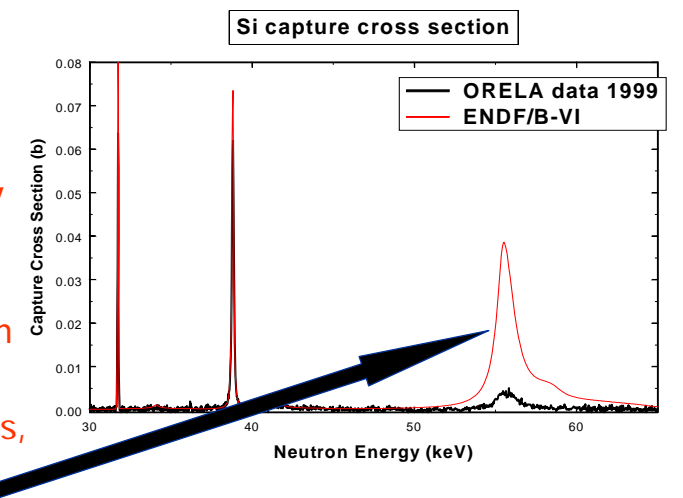

Ex: Large neutron sensitivity of older measurements led to many e rroneously-large resonance areas in current evaluations.

OAK Ridge National Laboratory

\begin{abstract}
$+2$
\end{abstract}

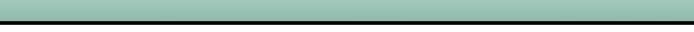

\title{
Neutron Sensitivity I
}

- Troublesome background that can result in measured capture Kernels being too large.

Caused by neutrons scattered from sample capturing in detector or environs within the time corresponding to the width of the resonance.

Sample dependent.

Very difficult to estimate or measure.

- "Me asured" for old $\mathcal{C}_{6} \mathcal{F}_{6}$ apparatus at $O R \mathcal{R E} \mathcal{L A}$ using carbon sample.

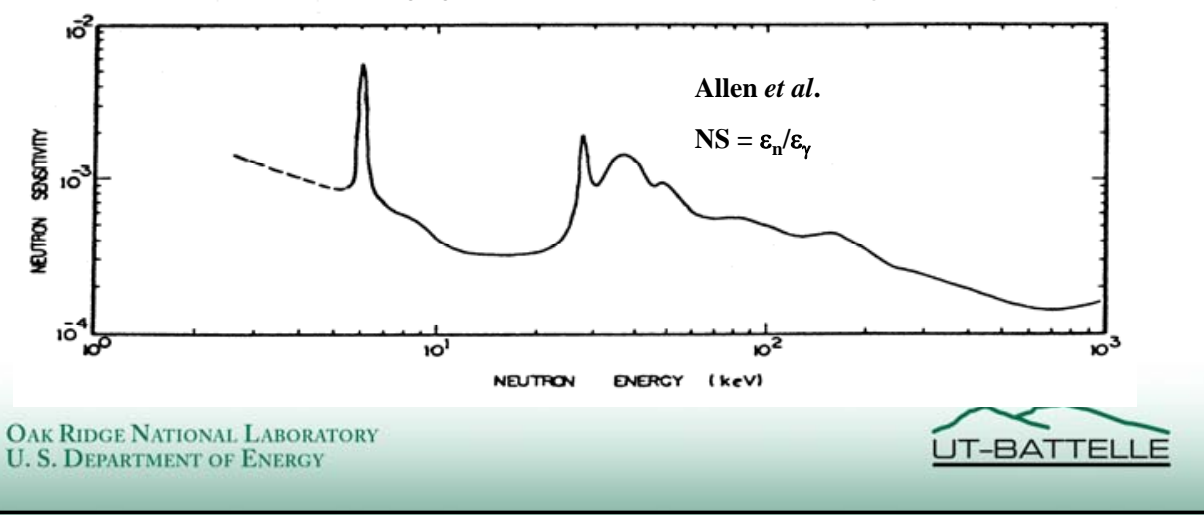


Modified capture data measurement system has significantly less structure

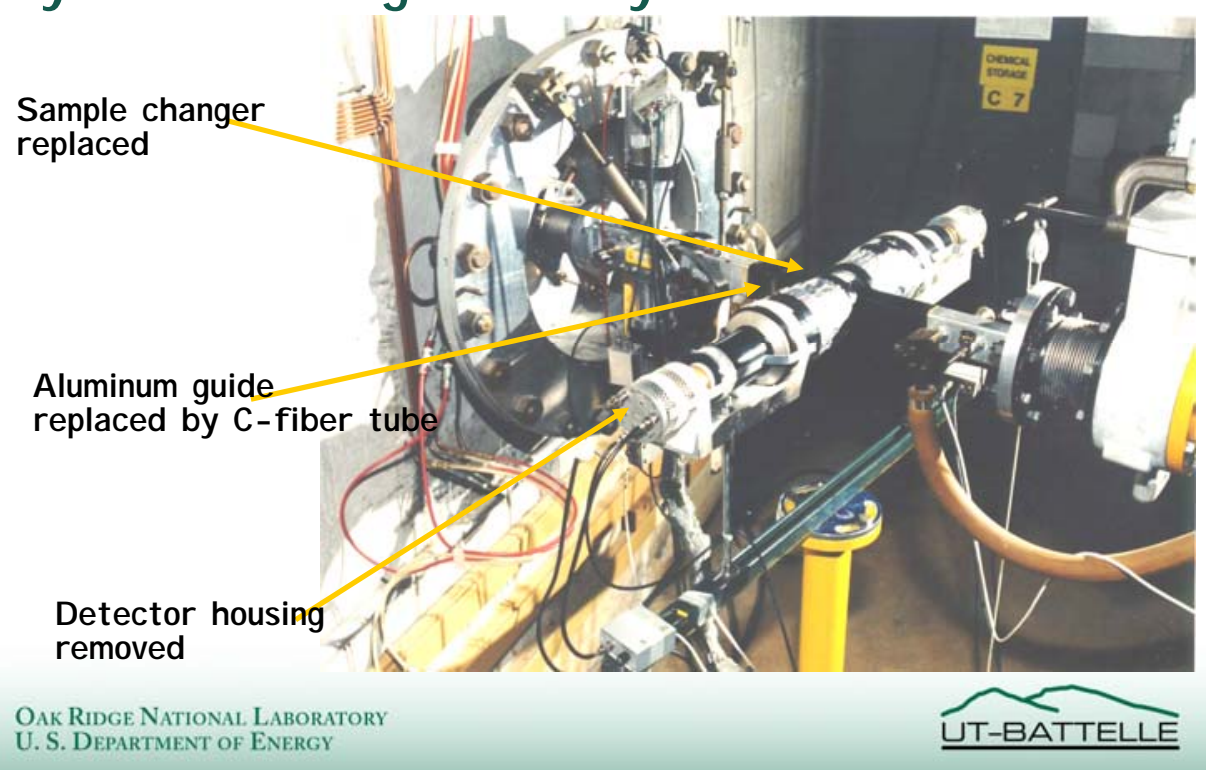

\section{Neutron Sensitivity I I}

- New ORELA data de monstrate that this correction was

underestimated in old $C_{6} \mathcal{F}_{6}$ data for resonances where $\Gamma_{n} \gg \Gamma_{\gamma}$.

- This background reduced in new ORELA $C_{6} \mathcal{D}_{6}$ apparatus to point where it is so far immeasurable.

- Ex: ${ }^{88} \mathcal{S} r$.

Resonances at 290,325 keV have $\Gamma_{n} / \Gamma_{\gamma}=18000$ and 20000 , respectively.

$\mathcal{N}$ ew resonance areas much smaller than previously reported even though new areas don't include a neutron sensitivity correction whereas old ones do.
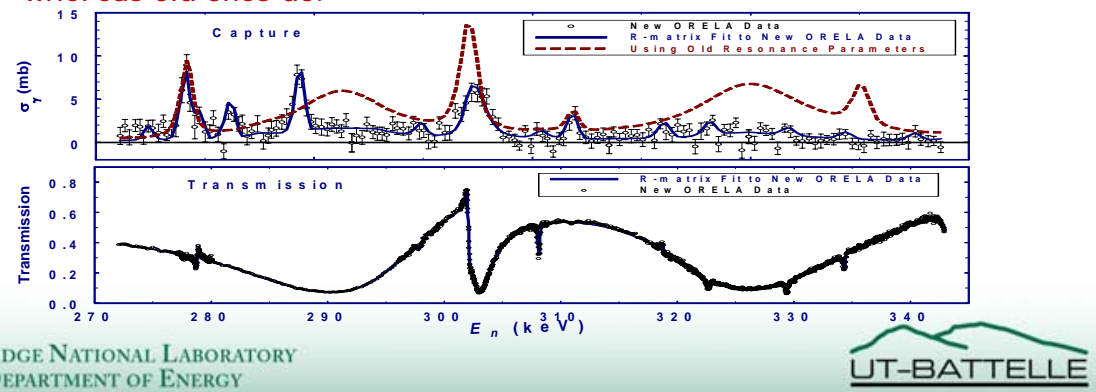


\section{Weigfting Function}

- Detector needs to have an efficiency independent of details of the capture cascade.

- $A 100 \%$ efficient $4 \pi$ calorimeter (e.g. a $\mathcal{B a F}_{2}$ ball) comes close to this ide al.

- But, it is expensive, complicated, and fias a larger ne utron sensitivity.

- $C_{6} \mathcal{D}_{6}$ detectors at $O R E \mathcal{R A}$ use the pulse height weighting technique.

- Simpler, cheaper, much reduced neutron sensitivity.

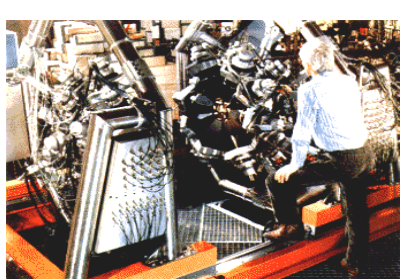

$4 \pi \mathrm{BaF}_{2}$ Detector

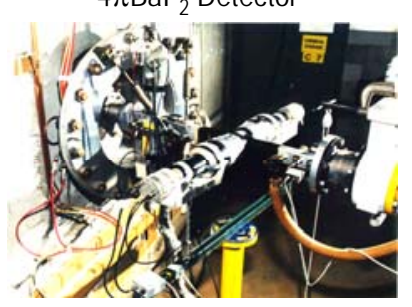

ORELA Pulse height weighting

Trouble with Weighting Functions for $\mathcal{C}_{6} \mathcal{D}_{6}$ Detectors

- $20 \%$ discrepancy found in neutron width of 1.15-KeV resonance in $\mathcal{F e}$ me asured with $C_{6} \mathcal{D}_{6}$ compared to transmission me as ure ments.

- Me asurements are done using a standard, us ually $\mathcal{A} u$ and $\mathcal{A g}$. Fe cascade is hard while those for Au and $\mathcal{A g}$ are soft.

- Corvi et al. (1988) showed that a me asured weighting function could solve the discrepancy.

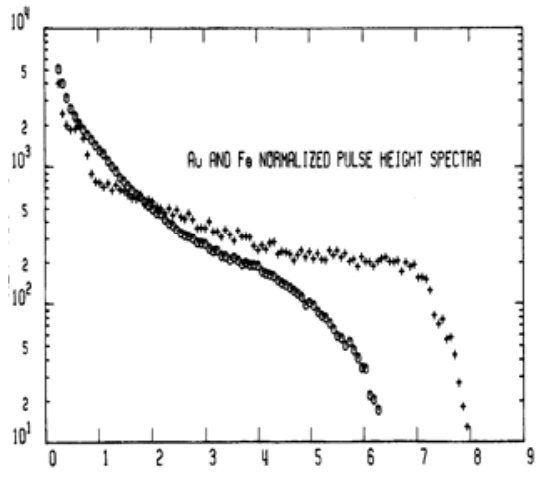

Perey et al. (1988) showed that weighting functions calculated using EGS 4 could solved $\mathcal{F e}$ discrepancy. Key finding: Sample and

surrounding material was not carefully described in previous weighting function calculations.

For each experiment the weighting function has to be calculated new. 
New ORELA Weighting Functions Demonstrated to be Accurate to Better Than $3 \%$
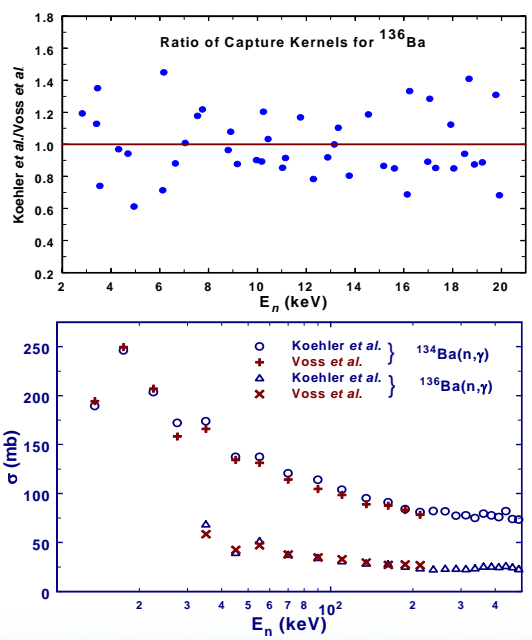

OAK RIDGe National. Laboratory

U.S. DEPARTMENT OF ENERGY
Excellent agreement between $O R \mathcal{R} \mathcal{A} C_{6} \mathcal{D}_{6}$ (Koefler et al.) and $\mathcal{F Z K B} \mathcal{B a F}_{2}$ (Voss et al.) $134,136 \mathcal{B a}(n, \gamma)$ me asure ments.

Hardness of cascade varies considerably from resonance. to-resonance, 6 ut no systematic difference between capture Kernels observed.

Excellent (<3\%) agreement for average cross sections.

\section{The Importance of Total Cross Section Data}

- More complete resonance parameter data will help improve nucle ar statistical model.

- Is indispensable for ob taining the most accurate $(n, \gamma)$ reaction rates. See resonances not visible in $(n, \gamma)$ data. Improved self-shielding and multiple scattering corrections.

- Lack of good total cross section data can le ad to serious errors in these corrections and hence in the cross sections.

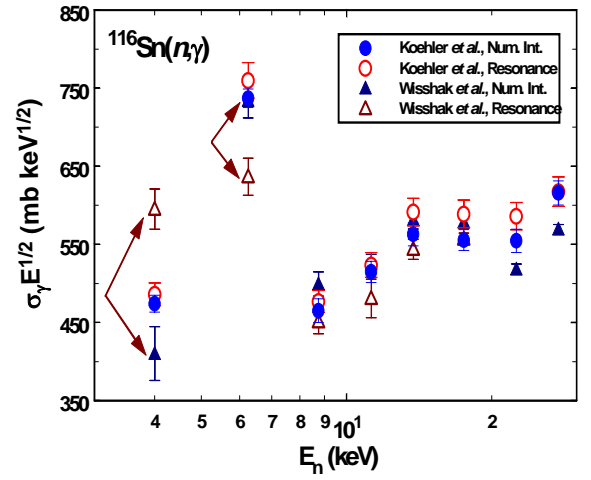

- Ex: ${ }^{116} S_{n}$ Use of incorrect neutron widths led to incorrect low-energy cross sections (Wisshak et al.). 
Simplified schematic of neutron transmission

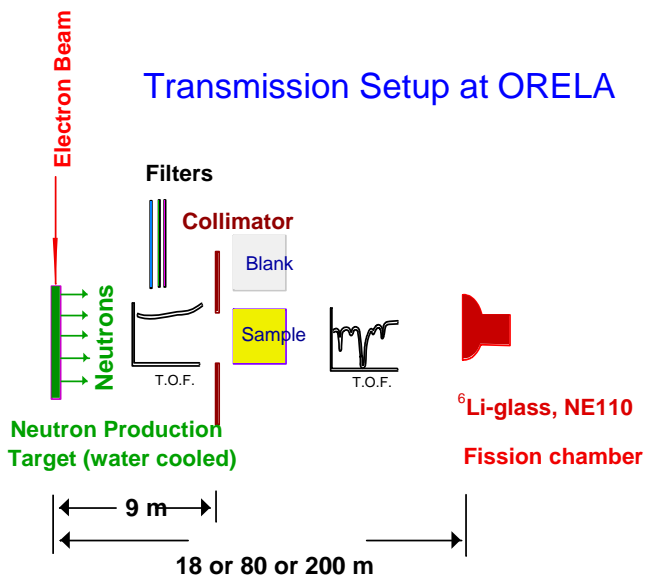

- For transmission, separate me asure ments of sample in and sample out:

$$
T=e^{-N \sigma_{T} d}
$$

- Additional background runs with filters are needed.

- Filters used to reduce frame-ove rlap background from lowenergy neutrons and to reduce $\gamma$-flash effects

Neutron Capture and Total Cross Section Experiments at a White $\mathcal{N e u t r o n ~ S o u r c e : ~ T y p i c a l ~ R e s u l t s ~}$
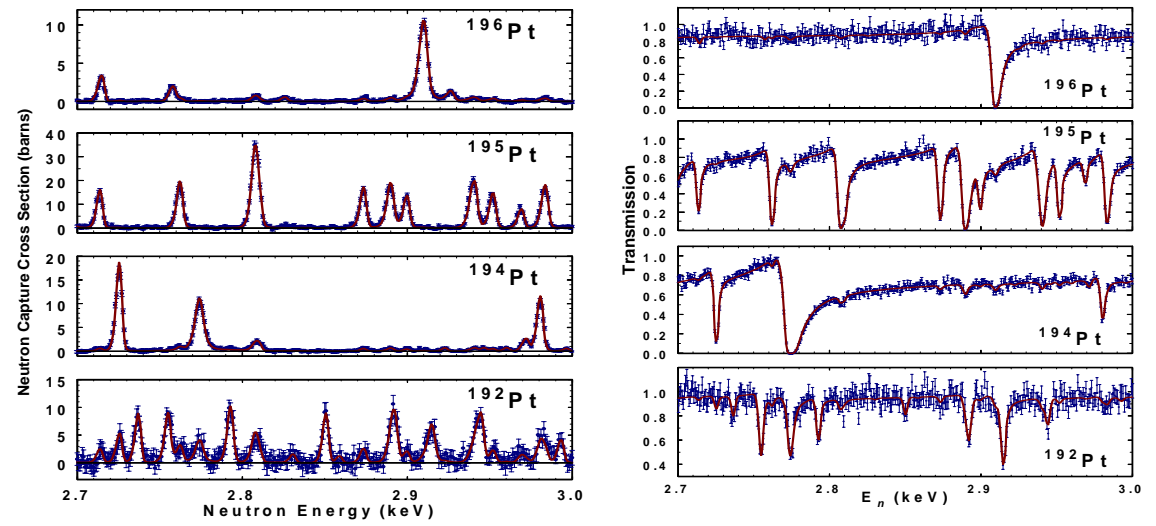

Only small part $(0.06 \%)$ of energy range of data shown.

Separate sample-out and background measurements needed. 
Excellent Resolution at ORELA Allows Better Resonance Analysis and Evaluations

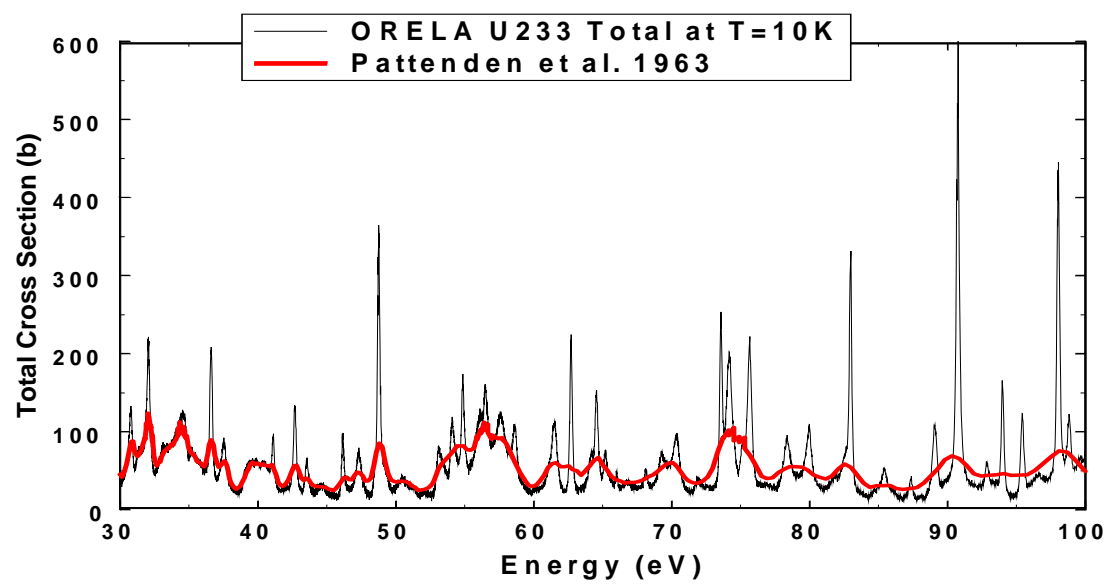

ORELA capture data compared to evaluations Several resonance areas are too large (neutron sensitivity).

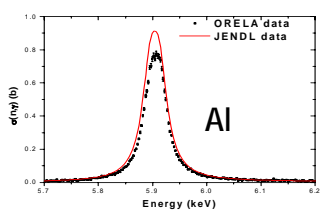

Energy (keV)

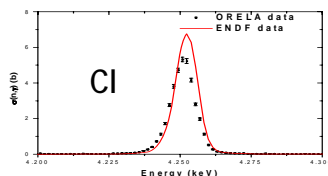

Energy (kev)

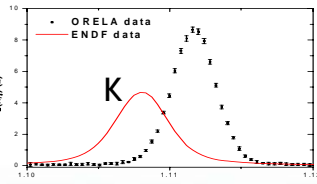

OAK Ridge National. LaBoratory

U.S. DEPARTMENT OF ENERGY
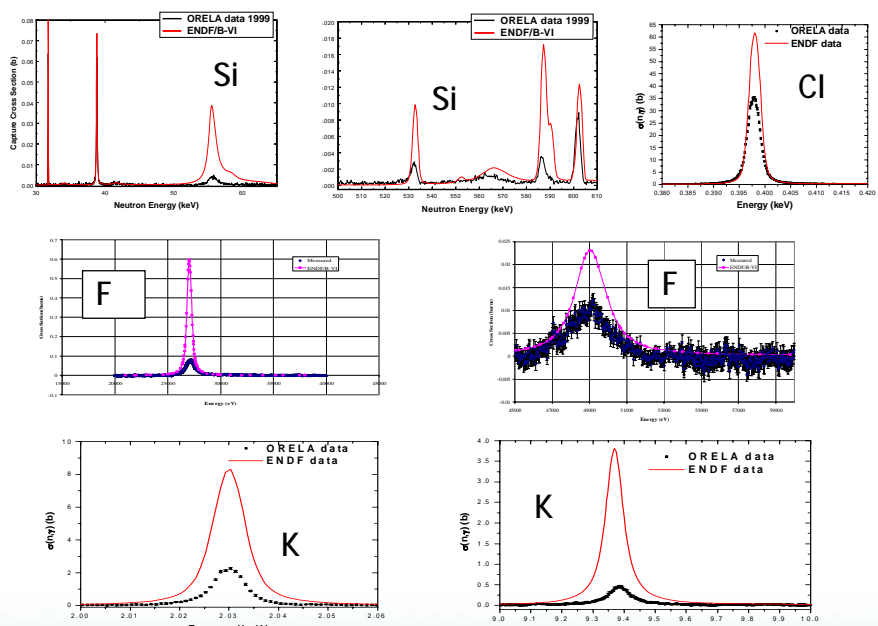

Energy (keV)
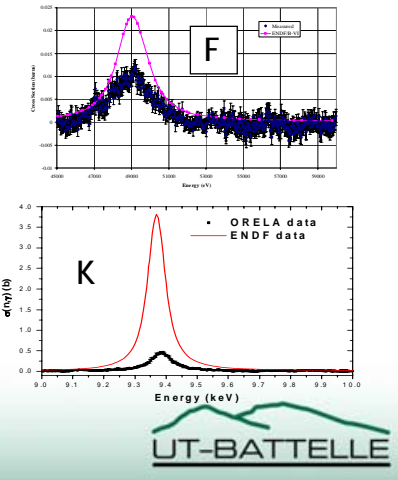


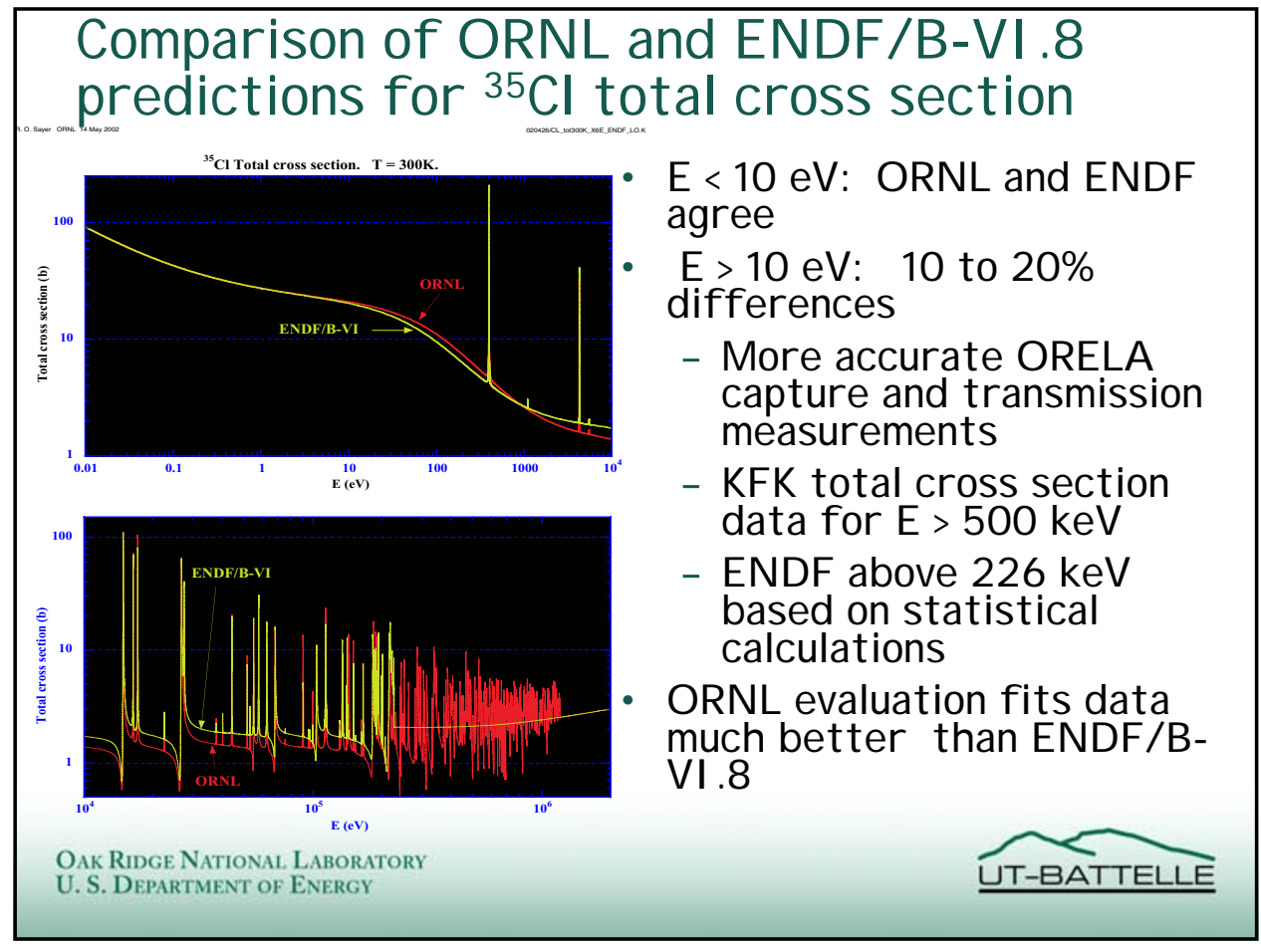

Comparis on of $S \mathcal{A M M Y}$ Fits with ORELA ${ }^{\text {nat }}$ Cl capture data
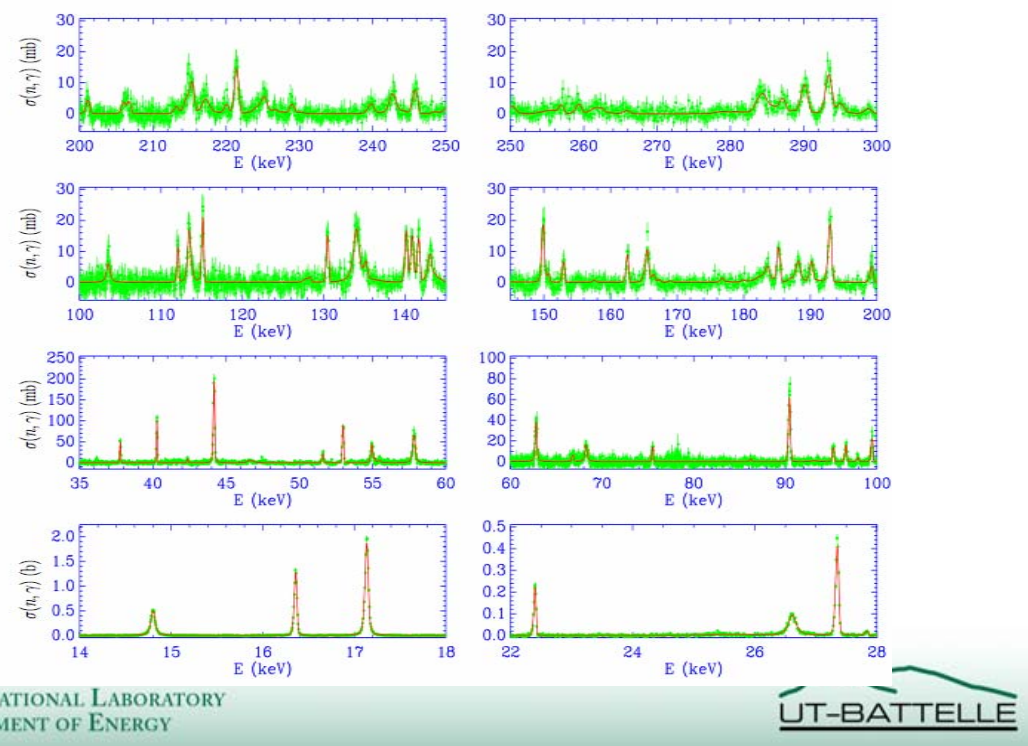

OAK RIDGE NATIONAL LABORATORY 


\section{Summary}

- Due to underestimated neutron sensitivity, incorrect weighting function, poor resolution, and limited energy range, some of the old capture data are in serious error. Old data are too large for many nuclides having small cross sections.

- New ORELA capture system has overcome these problems.

- Well known transmission set up produces accurate and reliable total cross section data.

Now possible to obtain highly precise and accurate nuclear data even for nuclides having very small cross sections. 



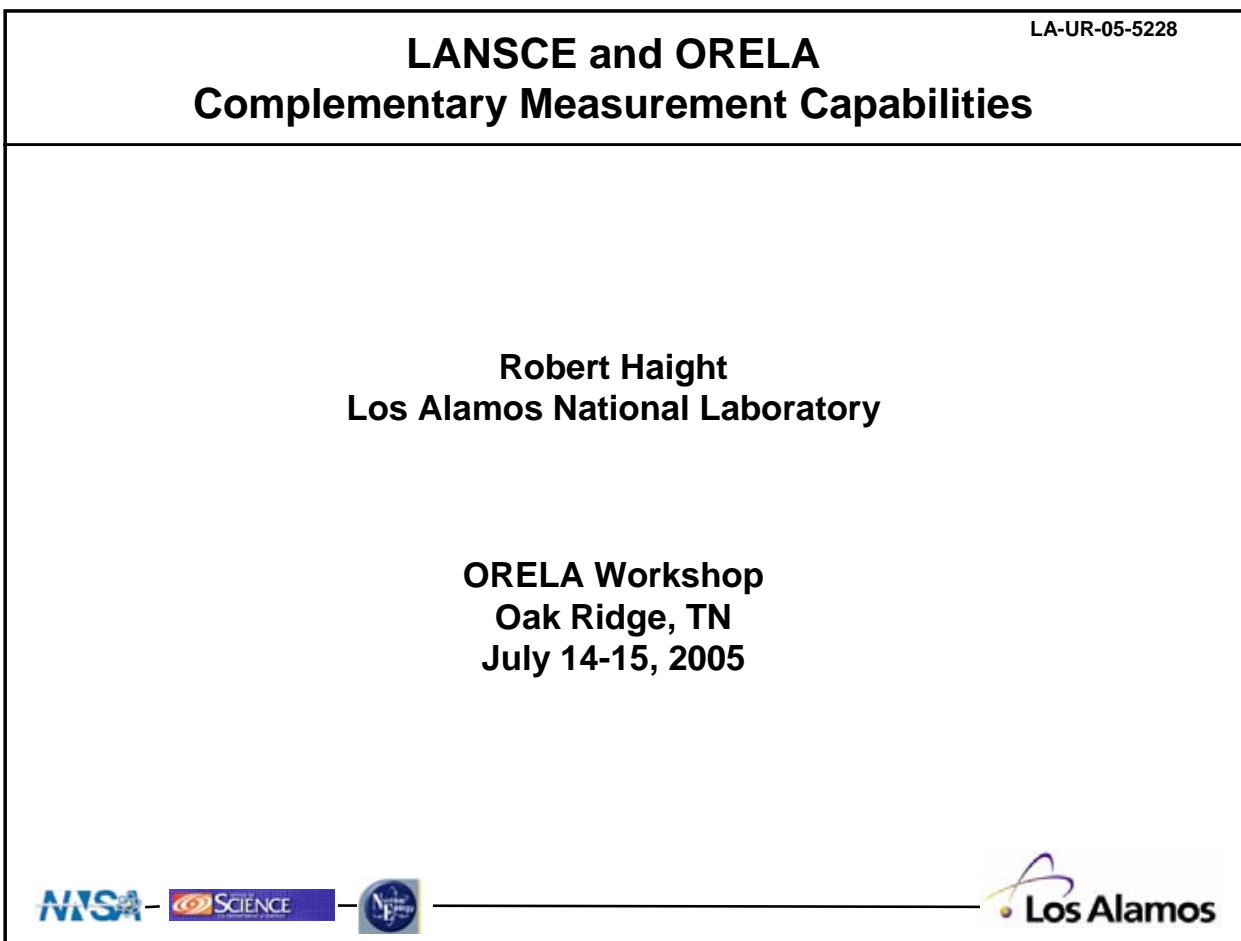

LA-UR-05-5228

\section{Outline}

- A little history

- Source comparisons

- Working together

- LANSCE-ORELA Complementarities

- Source characteristics

- Instruments

- People

- Missions (funding sources for LANSCE) 


\section{A little history}

- Studies of source characteristics

- Michaudon and Wender study of source characteristics (1990) - LA-UR-90-4355

- Peelle and Haight

- Koehler - NIM A460, 352 (2001) - for (n, $\gamma)$ on small samples

- Working together - some examples

- Haight (with Dickens) - Ta(n,xgamma)

- Keyworth - spins of resonances with polarized ${ }^{235} \mathrm{U}$

- Moore, deSaussure, Derrien - measurements/evaluations

- Nat Hill technology -e.g. PM tube bases

- Koehler @ LANSCE (Lujan)

- SAMMY - Larson

- People (Morgan $\longleftrightarrow$ Koehler)

Nuclear physics experiments at LANSCE use neutrons at four locations: Line B, Lujan Center, Target 2 and Target 4.

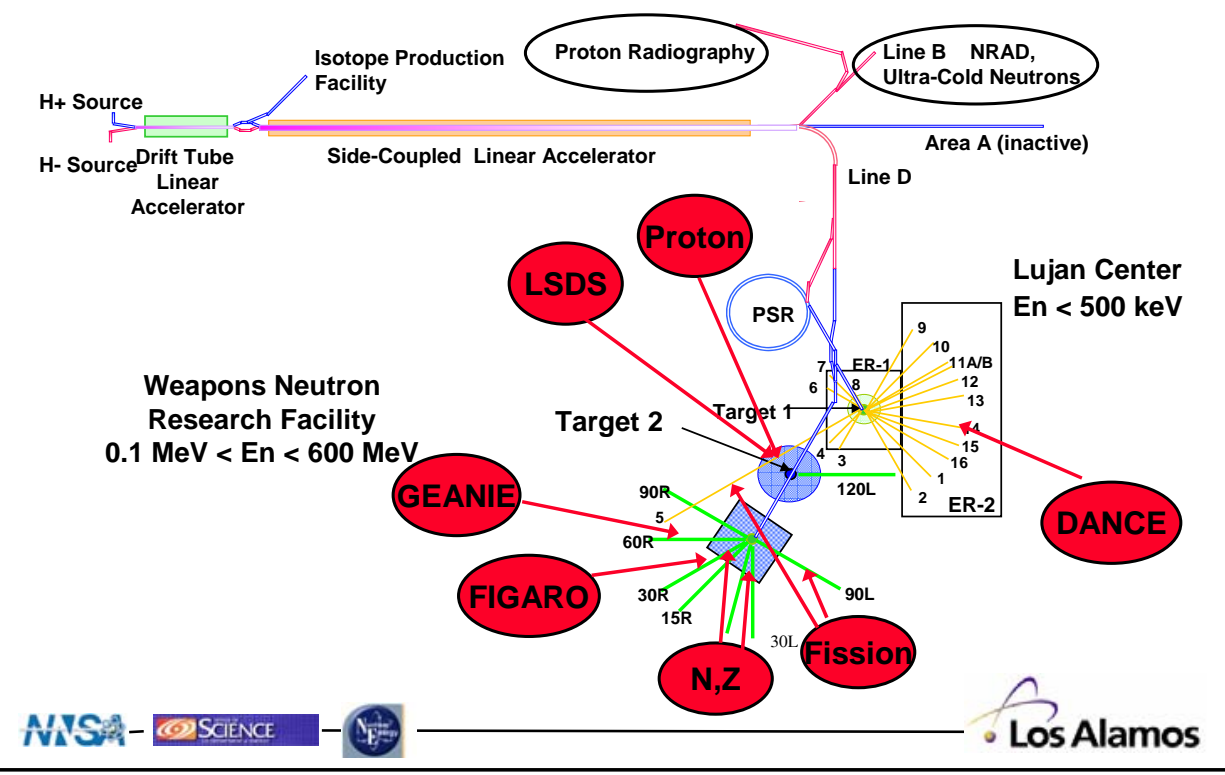


WNR -- Target Specifications

Target-2 (Blue Room)

Up to $\sim 1 \mu$ A proton beam

- Proton induced reactions

- 6 flight paths

- Proton irradiations

- Lead slowing-down

Target-4

spectrometer (LSDS)

$4 \mu \mathrm{A}$ proton beam for high-energy neutron spallation source. Typical operation is 35,000 pulses/sec with

$1.8 \mu \mathrm{sec}$ spacing.

- 6 neutron flight paths

- Neutron induced reactions

- Energy spectrum depends on flight-path angle

- Determine energy of neutron by time-of-flight

- Neutron irradiations

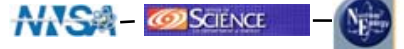
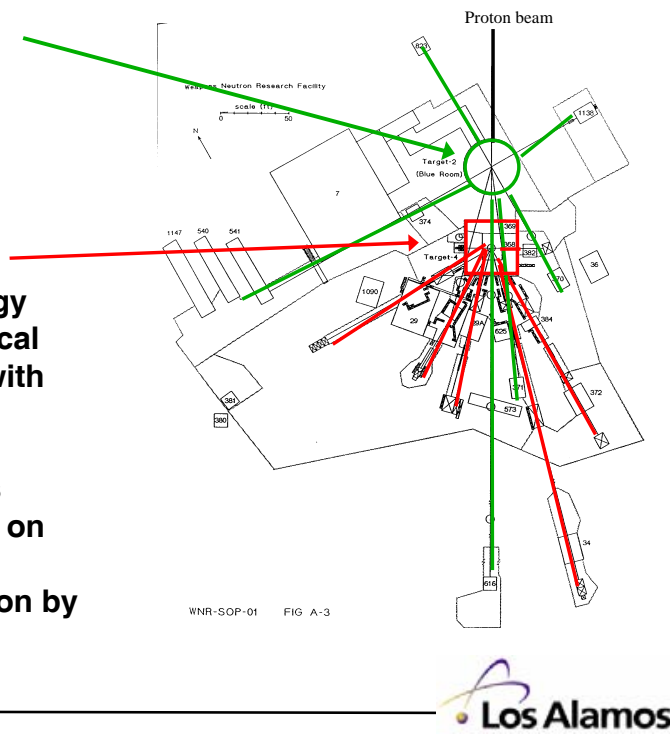

LA-UR-05-5228

\section{Lujan Center -- Moderated spallation source}

$800 \mathrm{MeV}$ protons + tungsten $->$ fast neutrons $(0.1$ to $800 \mathrm{MeV}$ )

$\ldots$ then fast neutrons $+\mathrm{H}_{2} \mathrm{O} \quad->$ slower neutrons (subthermal to $1 \mathrm{MeV}$ )

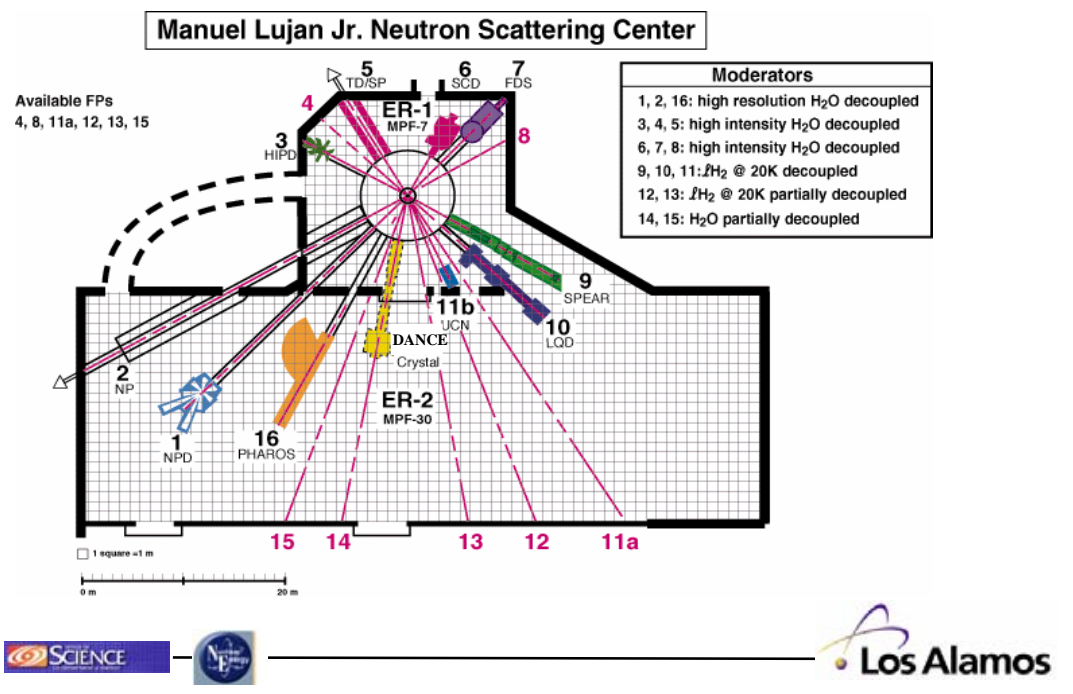


LANSCE neutron spectra cover energies from cold to several hundred MeV
Moderated source (Lujan)
Unmoderated source (WNR)
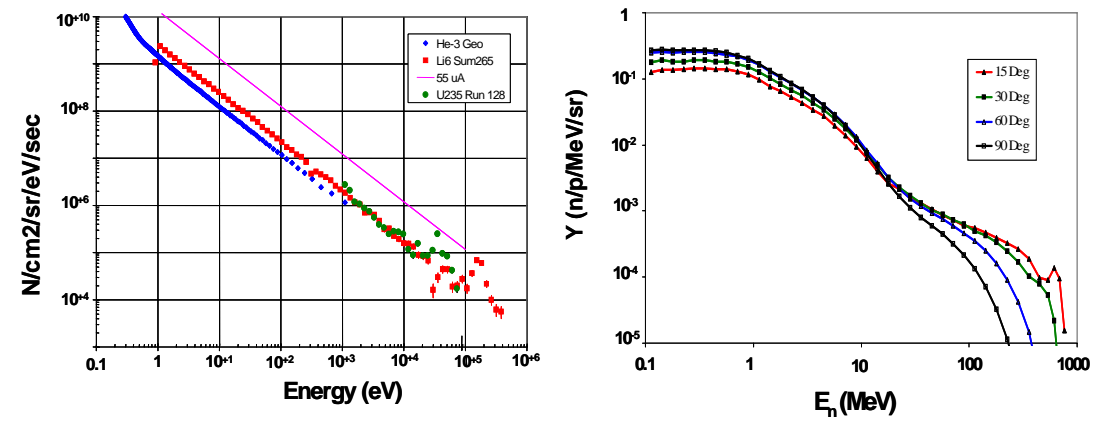

NNGA- @SAENCE

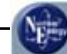

- Los Alamos

LA-UR-05-5228

Neutrons at LANSCE span 16 orders of magnitude in energy

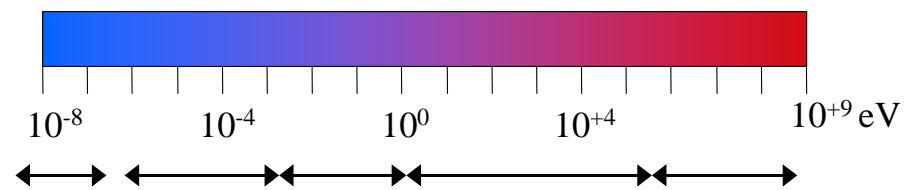

ultracold cold thermal epithermal fast

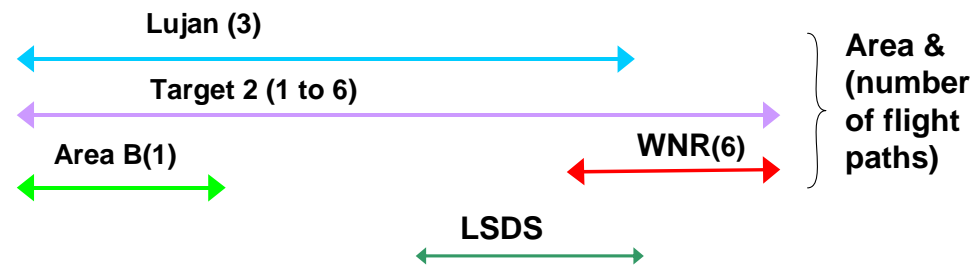




\section{Most measurements use the time-of-flight technique}

Time of flight over the flight path identifies the energy of the neutron that induces the reaction

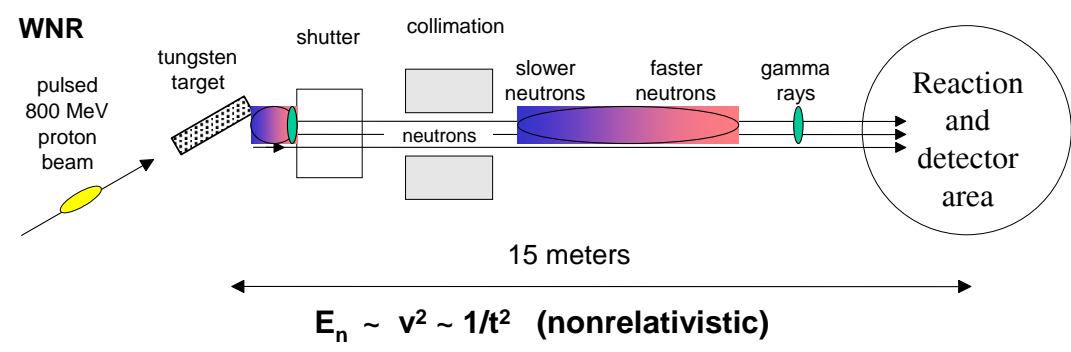

Important characteristics:

- Neutron flux at sample

- Repetition rate

- Flux spectrum

- "Gamma" flash

- Time resolution

- Available flight paths

- Backgrounds

\section{Comparisons show complementarity}

- Neutrons on sample

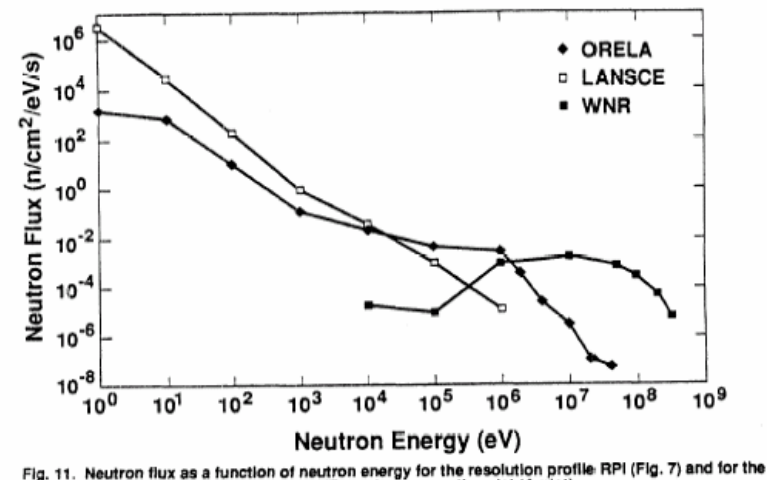

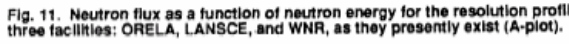

From: Michaudon and Wender (1990) 


\section{Resolution comparisons for epithermal neutrons}

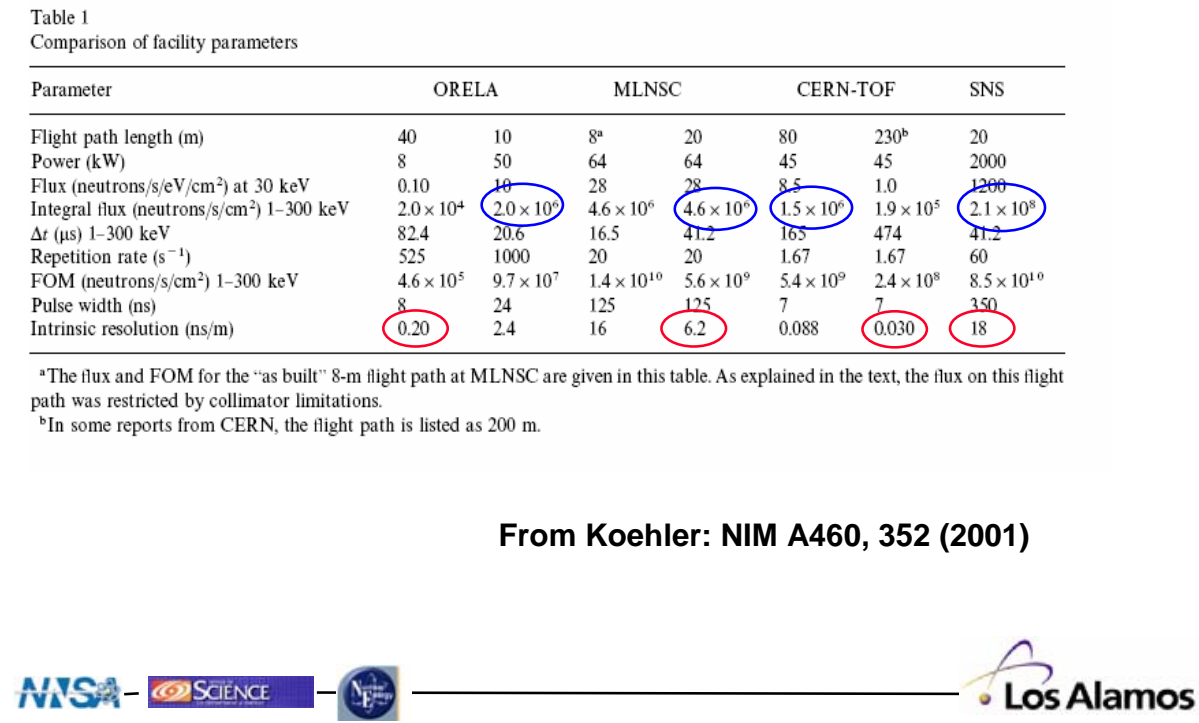

\begin{tabular}{|c|c|c|c|c|c|c|}
\hline 40 & 10 & $8^{a}$ & 20 & 80 & $230^{\mathrm{b}}$ & 20 \\
\hline 8 & 50 & 64 & 64 & 45 & 45 & 2000 \\
\hline 0.1 & & 28 & & & 1.0 & \\
\hline 2.0 & $\left(2.0 \times 10^{2}\right)$ & $4.6 \times 10^{6}$ & & $1.5 \times 10$ & $1.9 \times 10^{5}$ & \\
\hline 82.4 & 20.6 & 16.5 & 41.2 & 165 & 474 & 412 \\
\hline 525 & 1000 & 20 & 20 & 1.67 & 1.67 & 60 \\
\hline $4.6 \times 10^{5}$ & $9.7 \times 10^{7}$ & $1.4 \times 10^{10}$ & $5.6 \times 10^{9}$ & $5.4 \times 10^{9}$ & $2.4 \times 10^{8}$ & $8.5 \times 10^{10}$ \\
\hline 8 & 24 & 125 & 125 & 7 & & 2 \\
\hline 0.20 & 2.4 & 16 & & 0.088 & 0) & \\
\hline
\end{tabular}

"The flux and FOM for the "as built" 8-m flight path at MLNSC are given in this table. As explained in the text, the flux on this flight path was restricted by collimator limitations.

' In some reports from CERN, the flight path is listed as $200 \mathrm{~m}$.

From Koehler: NIM A460, 352 (2001)

ORELA and LANSCE Nuclear Physics Instruments

ORELA

Total cross section detectors

- ${ }^{6}$ Li glass

- Organic scintillators

Neutron capture

- $\mathrm{C}_{6} \mathrm{D}_{6}$

- $\mathrm{BaF}_{2}$-- 14 detector array

(n,alpha) -

- Gridded ion chamber -also used a compensated ion chamber

Others
LANSCE

Fast neutrons (En > $0.1 \mathrm{MeV}$ )

- GEANIE - gamma-ray detector array of 26 HPGe detectors

- FIGARO - neutron detector array

- Frisch-grid fission ion chamber

- $\quad(n, z)$ chamber - silicon + CsI(TI)

- Total cross section detectors (presently not active)

Epithermal

- DANCE - 4-pi calorimeter for neutron capture- $160 \mathrm{BaF}_{2}$ detectors

- Frisch-grid fission ion chamber

- LSDS: fission chambers, etc. 
Nuclear data measurements at LANSCE are made with several instruments

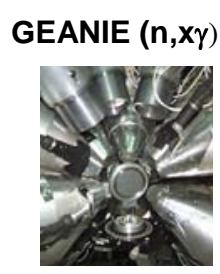

$\mathrm{N}, \mathrm{Z}$ (n,charged particle)

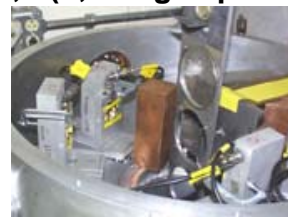

FIGARO $(n, x n+\gamma)$
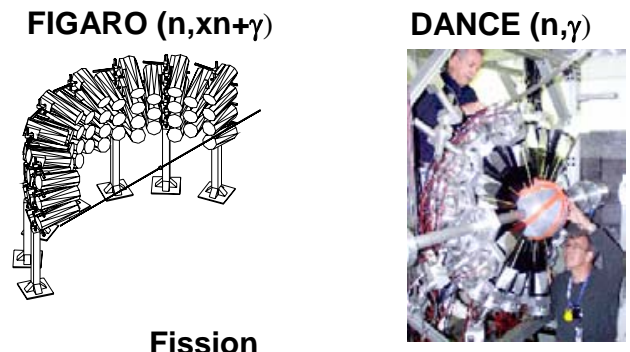

Double Frisch-gridded fission chamber; also standard fission ion chamber

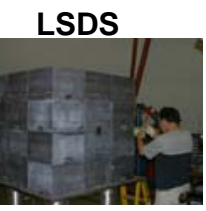

Total cross sections

NNG - OSSIENCE - BBF - Los Alamos

WNR neutron total cross sections were measured with $\sim 1 \%$ absolute uncertainty in the $5-560 \mathrm{MeV}$ range

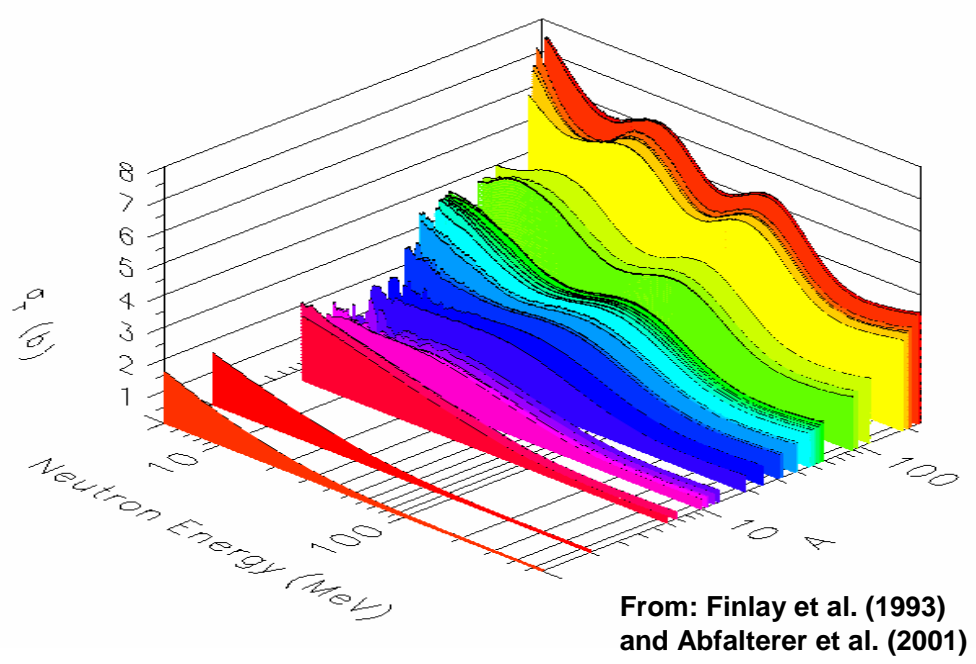




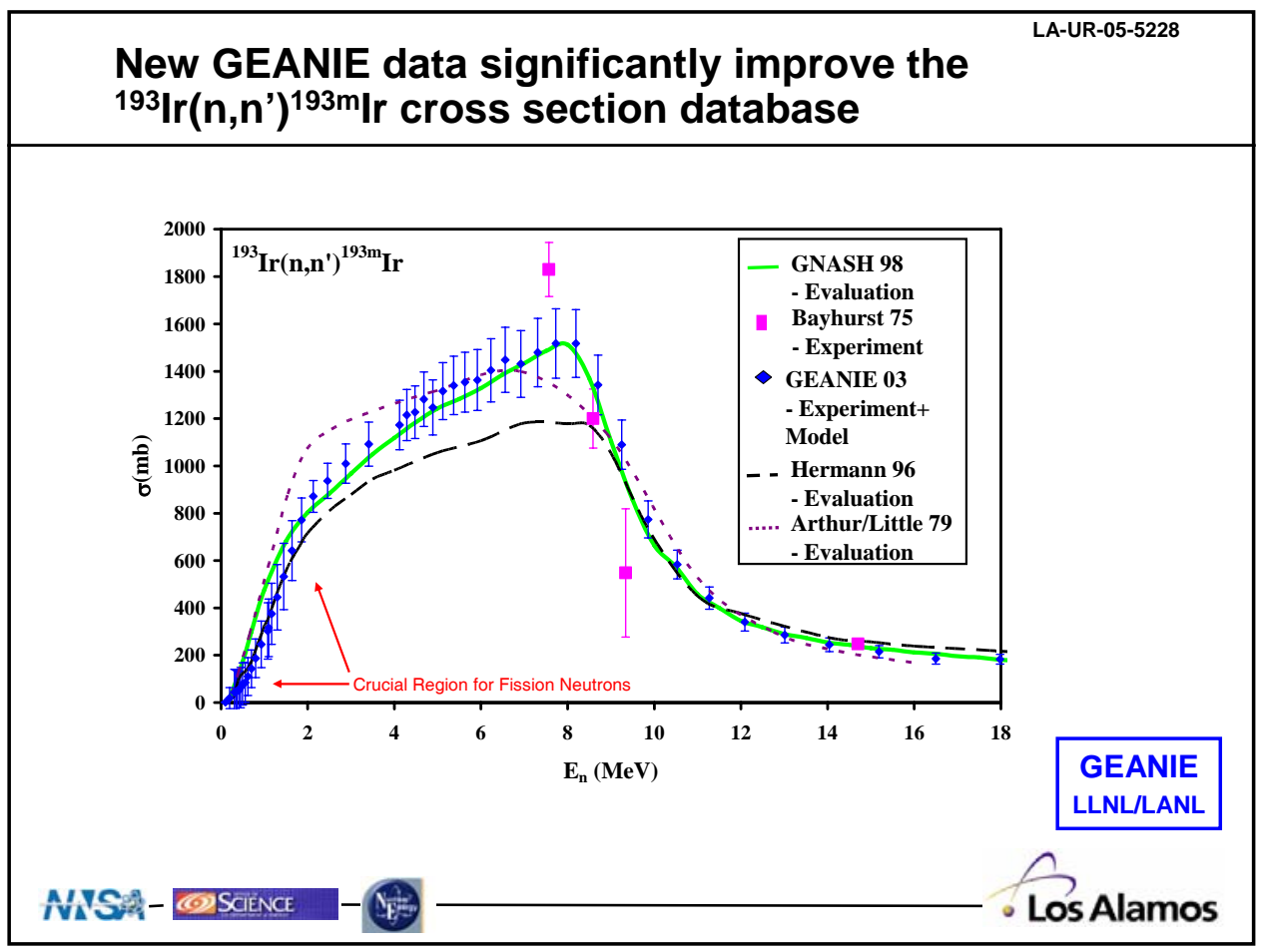

FIGARO data quantify how the fission neutron

LA-UR-05-5228 spectrum varies with incident neutron energy

${ }^{238} U(n, f)$ average neutron energy

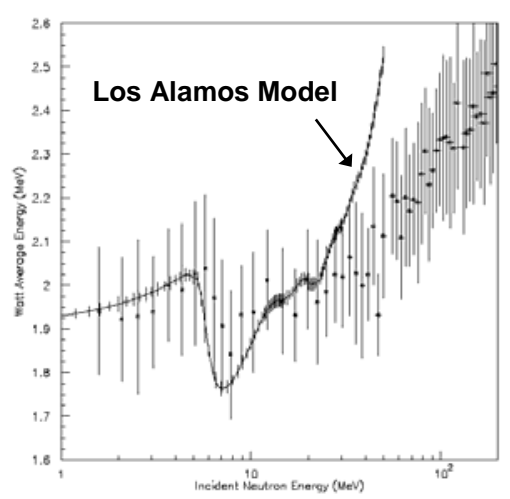

Agreement with Los Alamos Model is good below $20 \mathrm{MeV}$

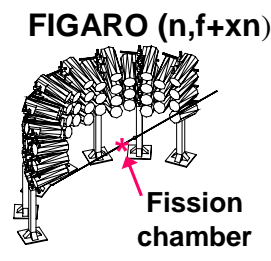

From: Ethvignot et al.,

Phys. Lett. B575, 221 (2003). 
Neutron emission spectra are measured by triggering on gamma rays
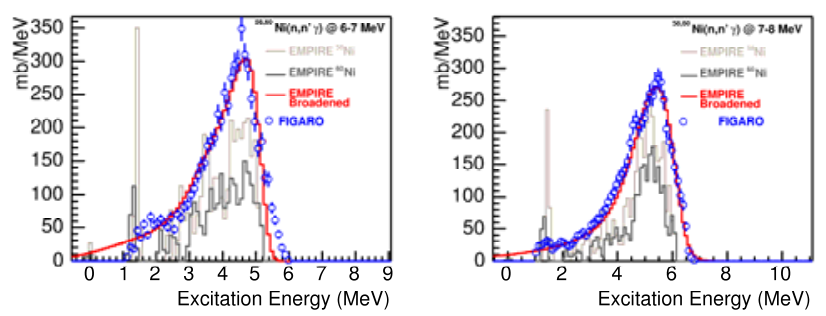

FIGARO $(n, x n+\gamma)$
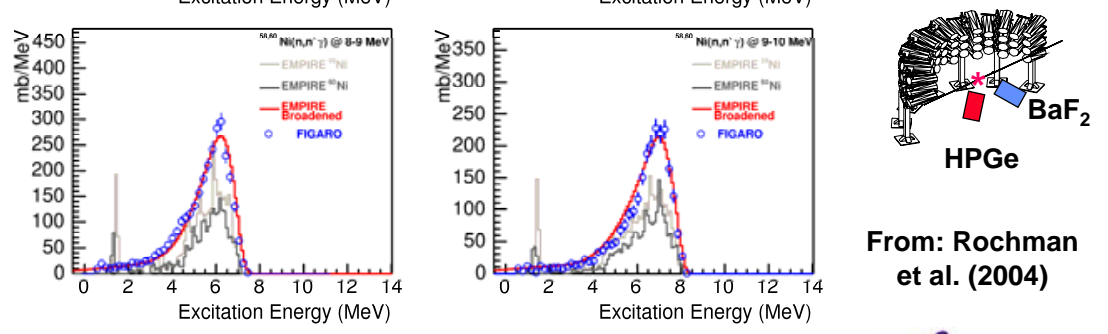

From: Rochman et al. (2004)

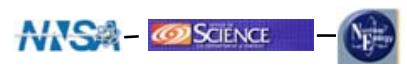
- Los Alamos

LANSCE $(n, z)$ data differentiate among different evaluations (guesses)

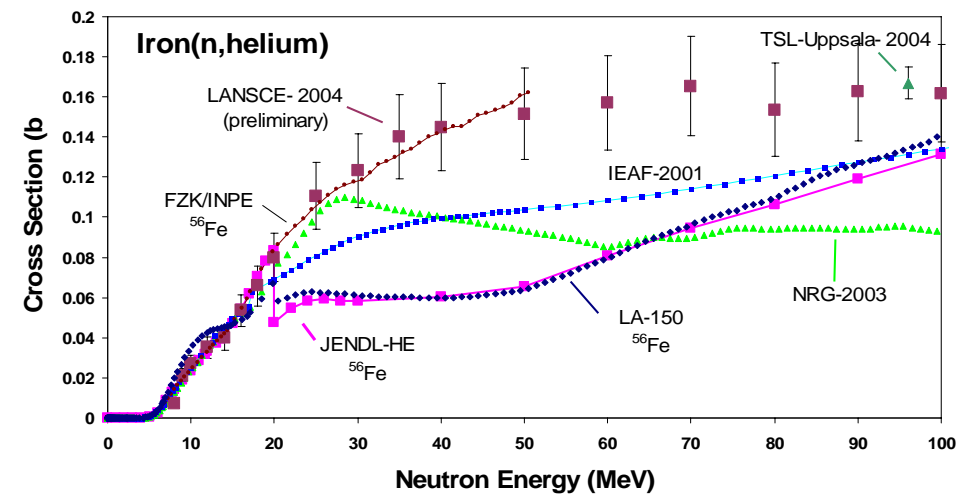

From: Haight, ANS meeting (2004) 
${ }^{237} \mathrm{~Np}(n, \gamma)$ data from DANCE is part of our AFCI program on actinides - sample is $0.44 \mathrm{mg}$

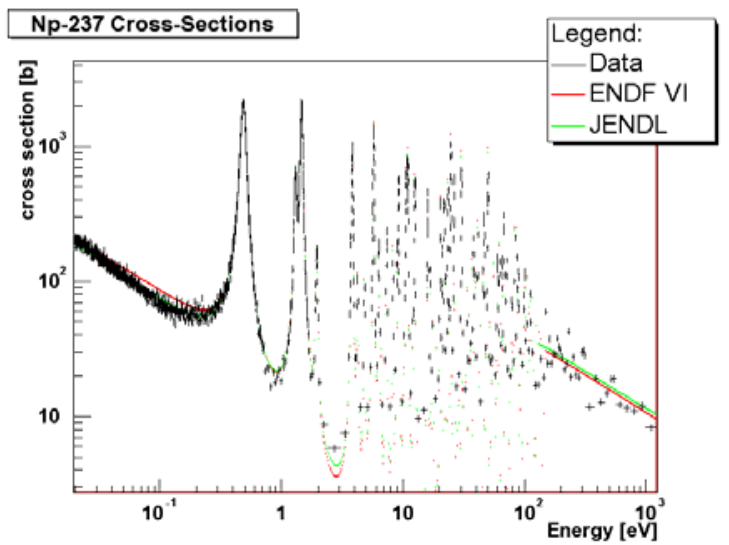

Target: $0.44 \mathrm{mg}{ }^{237} \mathrm{~Np}$ in $6.4 \mathrm{~mm}$ dia

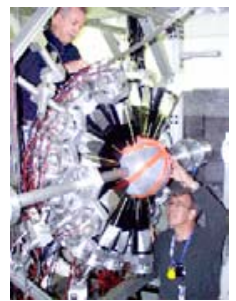

$\left(1.4 \mathrm{mg} / \mathrm{cm}^{2}\right)$

A Lead Slowing-Down Spectrometer is under development, driven by $800 \mathrm{MeV}$ protons from the PSR

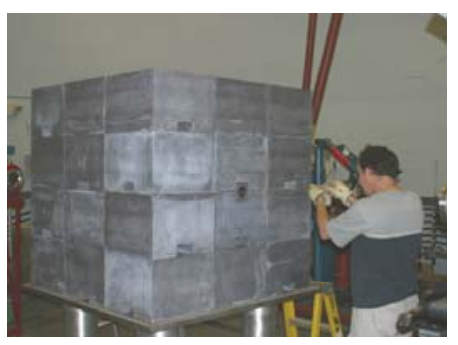

Neutron trajectories following the interaction of 1 proton with the tungsten target in the lead cube

development, driven by $800 \mathrm{MeV}$ protons from the PSR
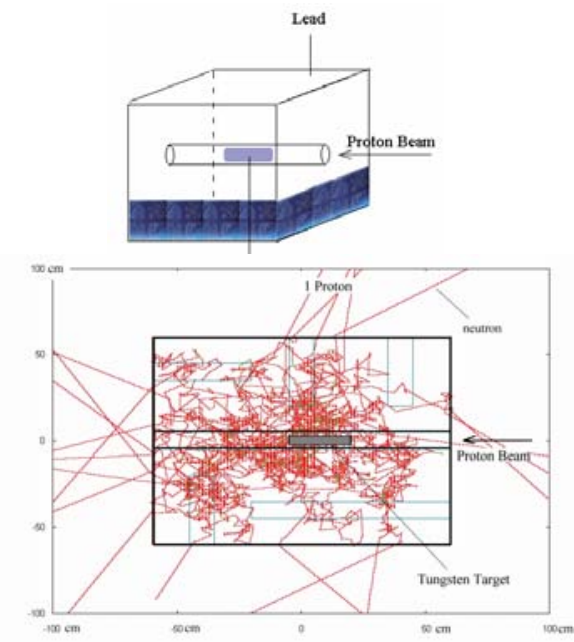


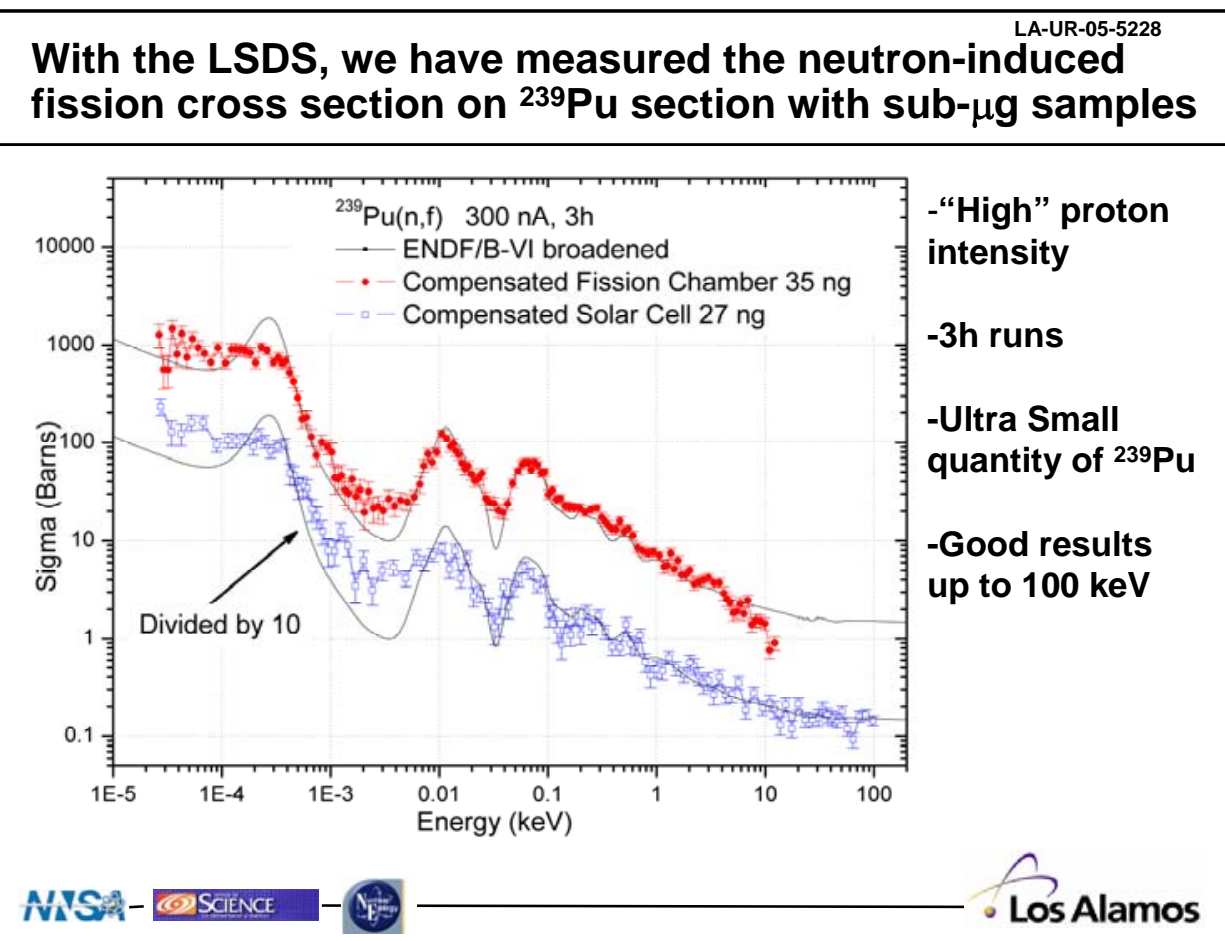

We can now measure fission cross sections with samples smaller than $10 \mathrm{ng}$

- Ran $1 \mu$ A of PSR beam to LSDS radiation levels $<2 \mathrm{mR} / \mathrm{hr}$ outside of Blue Room

$\rightarrow$ New shielding in BR hall

- Ran PSR at $30 \mathrm{~Hz}$ successfully

$\rightarrow$ First $30 \mathrm{~Hz}$ PSR beam

- Measured neutron-induced fission cross section of ${ }^{239} \mathrm{Pu}$ with a sample of $9.87 \mathrm{ng}$. Results agree with broadened ENDF/B-VI.

$\rightarrow$ This meets our goal for measuring the fission cross section of the ${ }^{235} \mathrm{U}$ isomer with a $10 \mathrm{ng}$ sample.

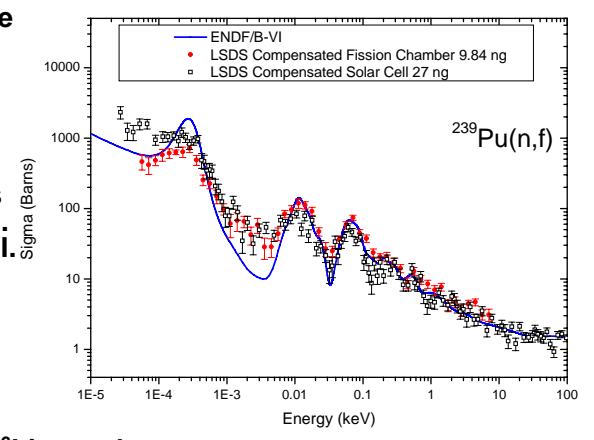

- Measured $(n, \alpha)$ cross section with small ${ }^{6} \mathrm{Li}$ sample

$\rightarrow$ Good prospects for other $(n, \alpha)$ measurements.

- DAQ works well in present configuration. Digital filtering under development. 
Fission cross section measurements are underway

- Extend program of Lisowski and that of Staples \& Morley

- Measurements both at WNR and at Lujan $\rightarrow$ data from thermal to $\sim 200 \mathrm{MeV}$

- Double Frisch-gridded ion chamber - from Hambsch (IRMM)

- Data soon

The LANSCE program in nuclear data involves many laboratories

- GEANIE - LANL, LLNL, Bruyères-le-Châtel, NC State

- FIGARO - LANL, Bruyères-le-Châtel

- N,Z - LANL, Ohio U

- DANCE - LANL, LLNL, ORNL, Colorado School of Mines, FZK Karlsruhe

- LSDS - LANL, LLNL, BNL, Bruyères-le-Châtel, RPI

- Fission - IRMM (Geel)

- Proton - LANL, Georgia Tech

- Others - MIT, Kentucky, Kyushu, Harvard,... 
The LANSCE program is supported by several funding agencies

- NNSA - accelerator and experiments

- DOE - Nuclear Energy - Advanced Fuel Cycle Initiative

- DOE - Office of Science - Nuclear Physics

- LANL internal funds (LDRD)

\section{ORELA and LANSCE have complementary capabilities}

- ORELA

- Excellent resolution in resonance region

- Well understood source term (SAMMY)

- Neutron flux larger in $\mathbf{5 0} \mathrm{keV}$ to a few $\mathrm{MeV}$ region

- Many well-characterized instruments

- Many flight paths

- (Ability to do classified experiments)

- LANSCE

- Excellent timing for fast neutrons ( $<1 \mathrm{~ns}$ at WNR)

- High intensity for En < $50 \mathrm{keV}$ (Lujan) and En > a few MeV (WNR)

- Energy range extends to $\sim 600 \mathrm{MeV}$

- Low gamma-flash for fast neutrons (WNR)

- Many well-characterized instruments

- Many flight paths - used simultaneously

- Ability to access proton beam directly (e.g. LSDS)

- (Ability to do classified experiments) 



\section{The Gaerttner LINAC Laboratory \\ Review and Current Activity}

Y. Danon and R.C. Block, M. Rapp and F. Saglime Rensselaer Polytechnic Institute, Troy, NY, 12180

and

J.A. Burke, N.J. Drindak, J.G. Hoole, G. Leinweber Lockheed Martin Corporation, Schenectady, NY 12301-1072

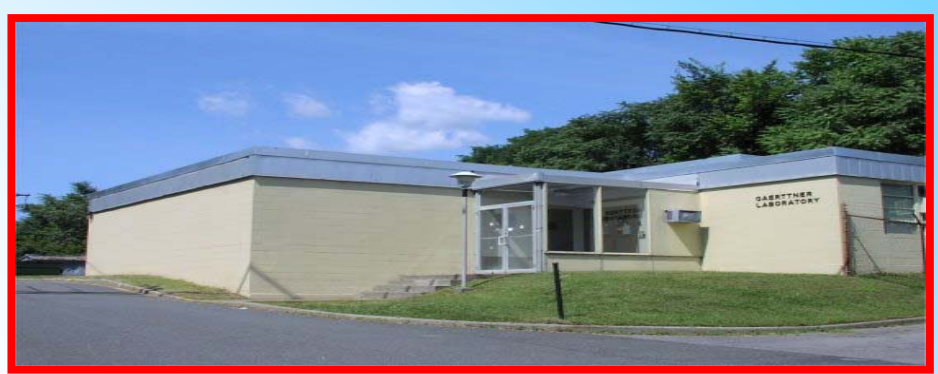

ORELA Workshop July 14-15, ORNL

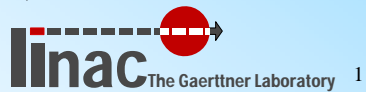

\section{History}

- The RPI LINAC started operation in December 1961

- Working "continuously" since
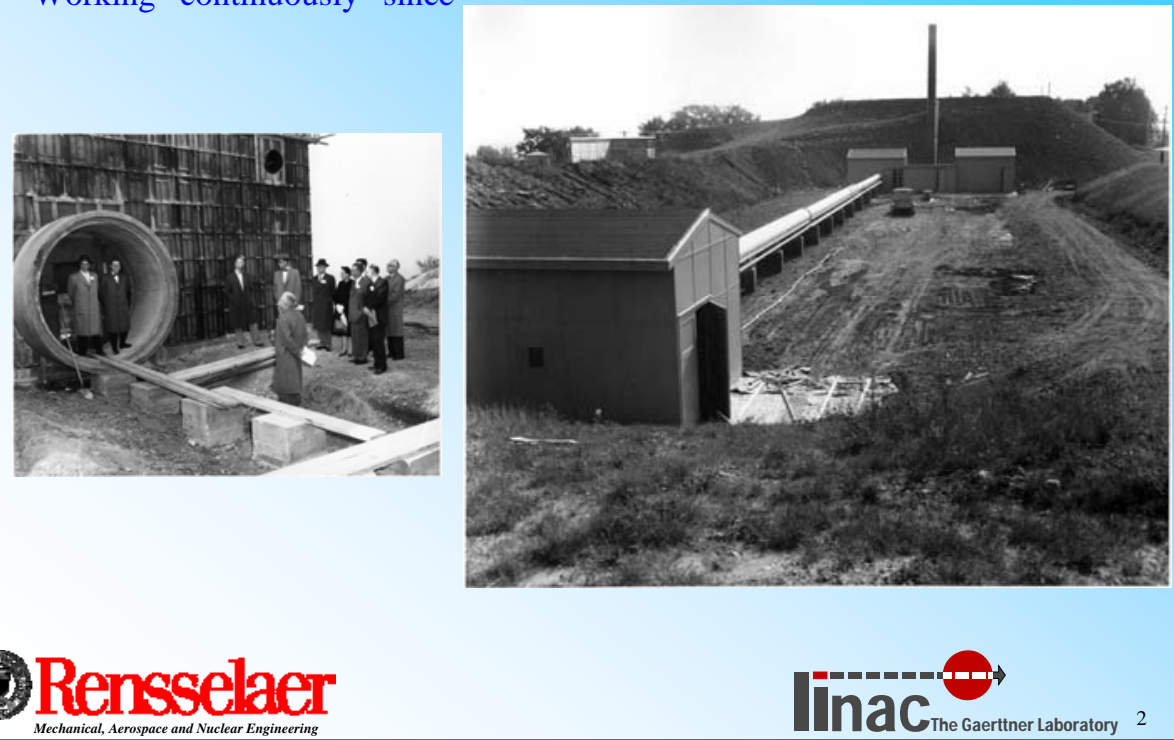


\section{Brief History of Past Activity}

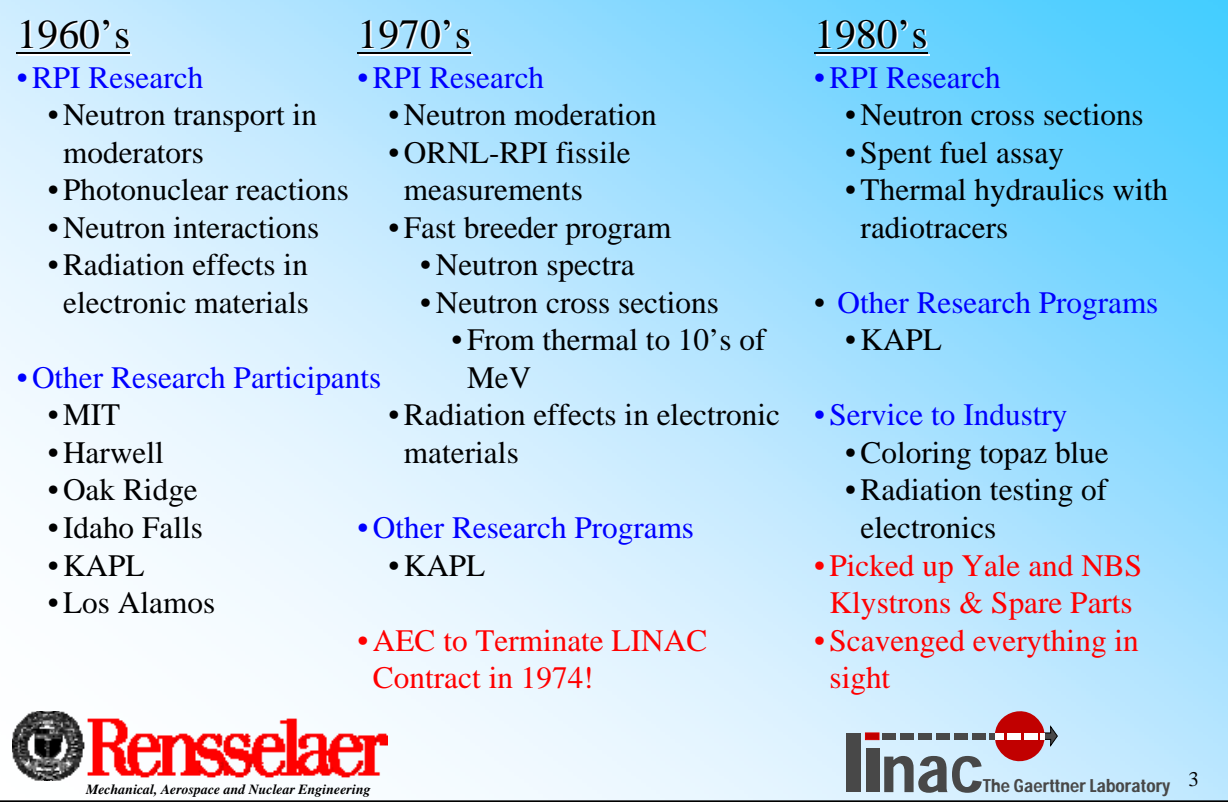

\section{Brief History of Past Activity cont.}

1990’s

-KAPL-RPI Research Program

- New state-of-the-art detectors

- New instrumentation

September 1997- LINAC Cited as

- Neutron cross sections

- Other Programs

- Supervoltage computed tomography

- Radiation effects in electronics

- Destruction of organic pollutants

- Service

- Detector calibration

- Radiation testing of electronics \& materials

\begin{abstract}
ANS Nuclear Historic Landmark "This was one of the first laboratories, utilizing a high-power electron linear accelerator, that generated accurate nuclear data for the design of safe and efficient nuclear power reactors."
\end{abstract}




\section{The 1990’s}

- Klystrons Wearing Out

- Picked up last klystron and parts from

Hanscom Air Force base

- Only a few more years left until klystrons fade away

- Facing "put up or shut down"

KAPL Investment

-LINAC Refurbishment

- Contract signed September of 1997

- \$1.4 million project

- New and rebuilt klystrons

- Improved RF system

- Reinstalled section \#9

- Completed in time for Y2K

\section{ORensselaer}

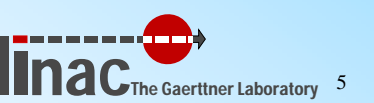

\section{Installation of Accelerator Section 9}
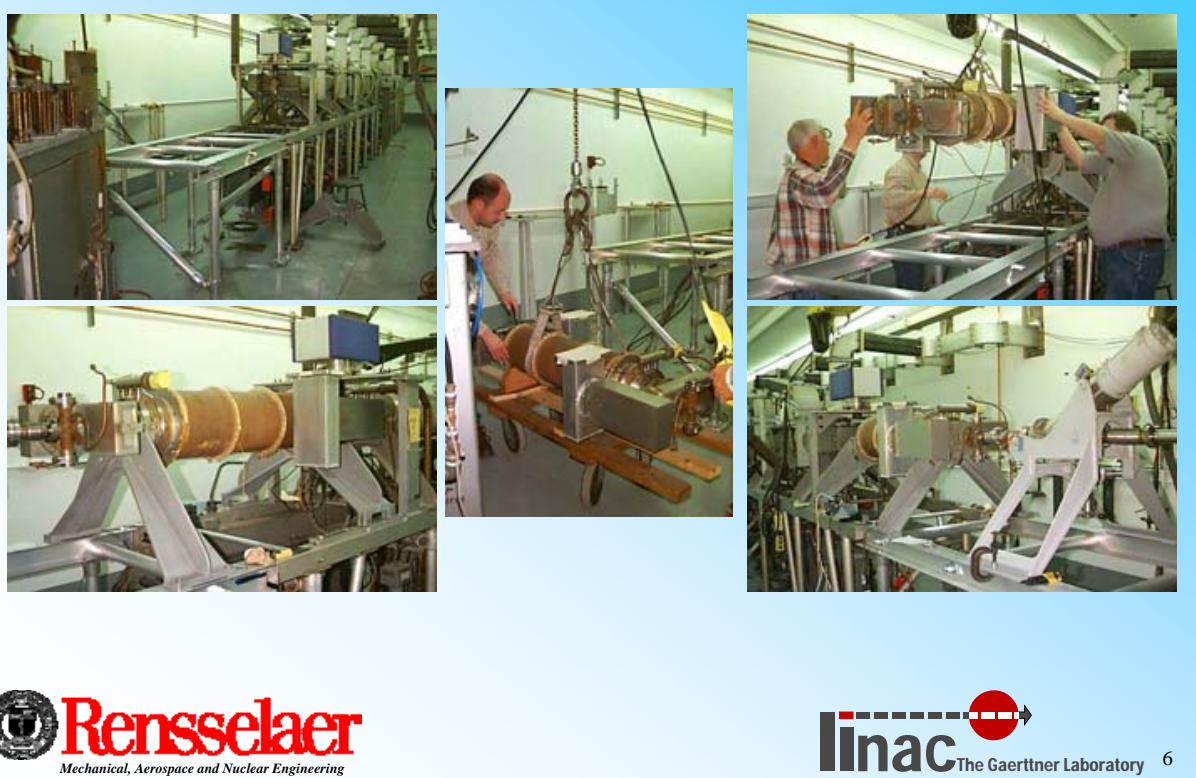


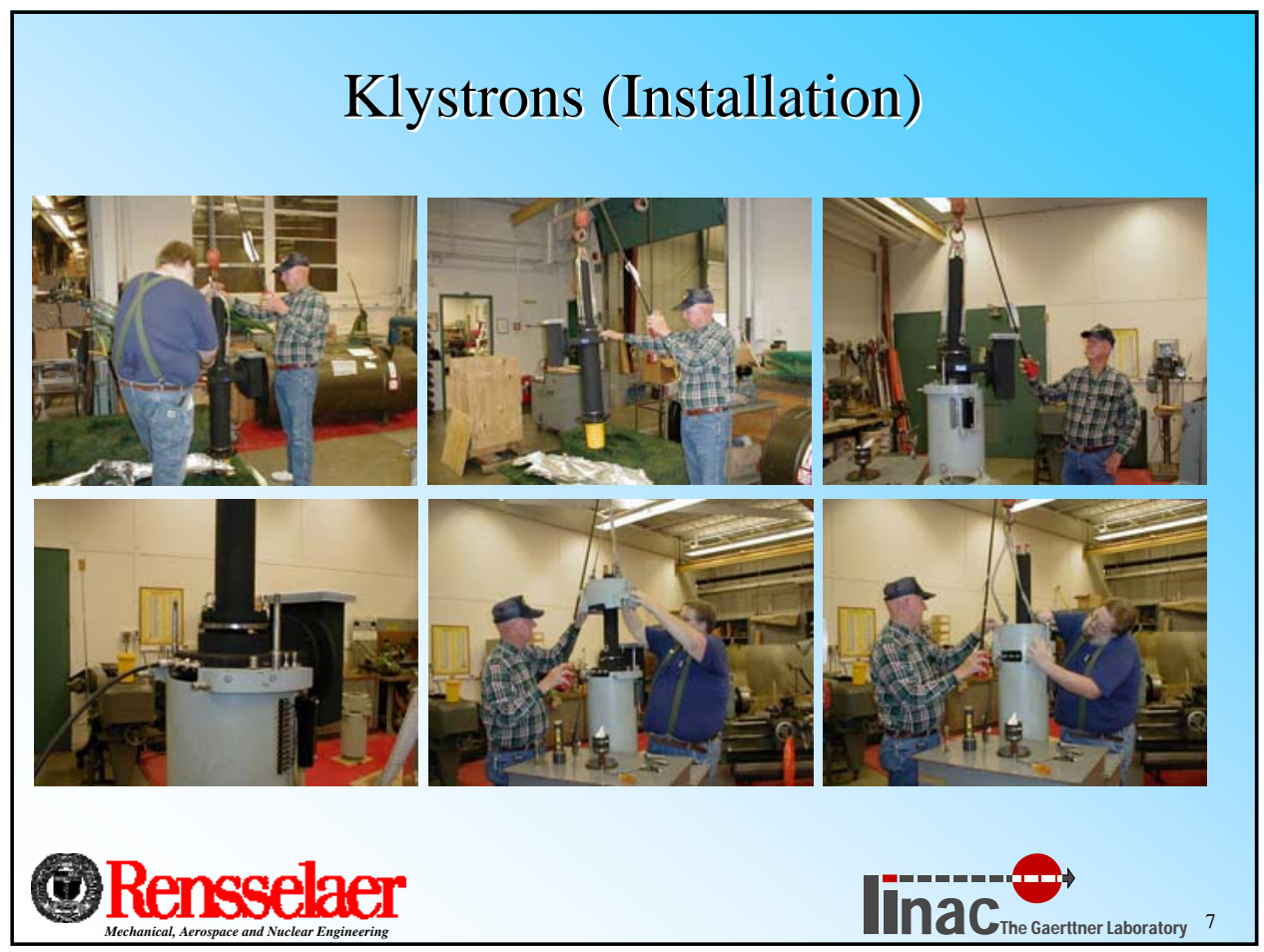

\section{Upgrade Summary (completed in 2000)}

-10 new klystrons were purchased and installed

- Service life extended by more than 10 years.

- Performance improvement:

- Overall beam power on target increased by $30 \%$ to $34 \%$

- Neutron production increased by $28 \%$ to $36 \%$

- Instrumentation:

- Network analyzer

- Beam measurement:

- Target pulse \& energy distribution

- Digital oscilloscope for klystron operation, tuning, and target pulse monitoring.

-Calibrated and balanced forward and reflected power monitors

- Arc detection and reverse power sensing of waveguide feedlines

\section{ORensselaer}

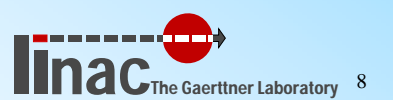



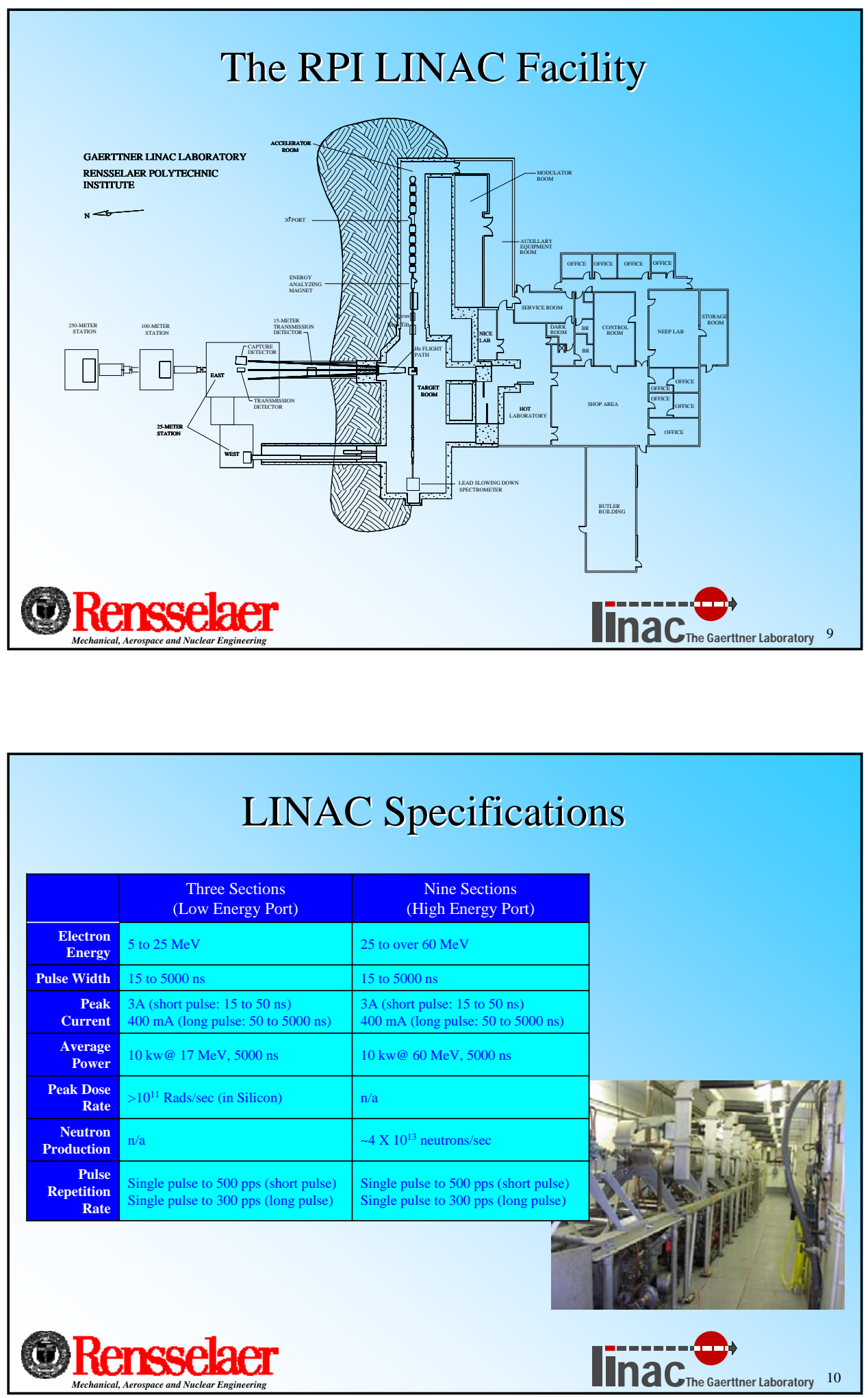


\section{Experimental Setups}

- Cross section measurements

- Flight Paths 15m, 25m, 100m and 250m

- Several Neutron Production Targets

- Lead Slowing Down Spectrometer

- Rabbit for sample activation

- X-Ray production target

- Low energy beam port for studies of radiation effects
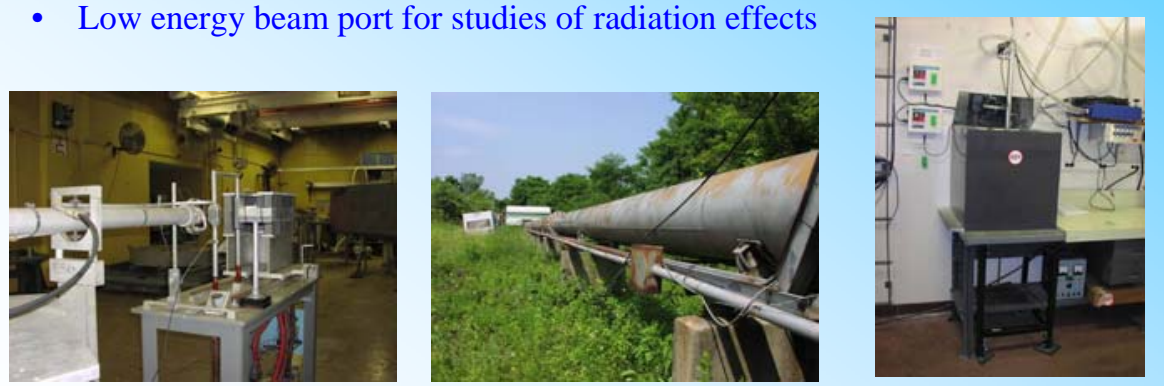

\section{ORensselaer}

\section{Setup For Cross Section Measurements}

- Li-Glass detectors for transmission measurements at $15 \mathrm{~m}$ and $25 \mathrm{~m}$

- 16 segment $\mathrm{NaI}(\mathrm{Tl})$ capture multiplicity detector for capture cross section measurements.

- Automated sample changers and beam blocks

- Time of flight clocks

- Neutron producing targets

- Lead slowing down spectrometer

- New experiments are being installed

- Data acquisition systems

- Data reduction systems

Many years of accumulated experience
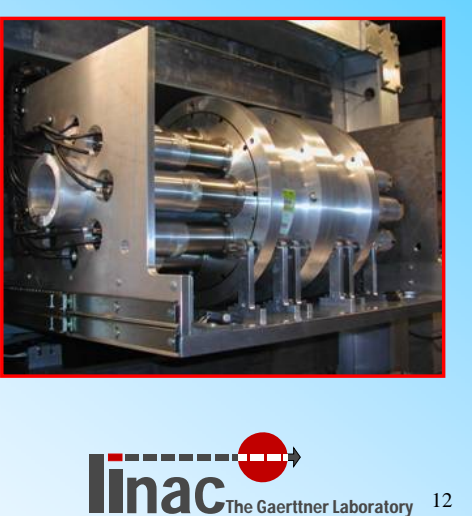


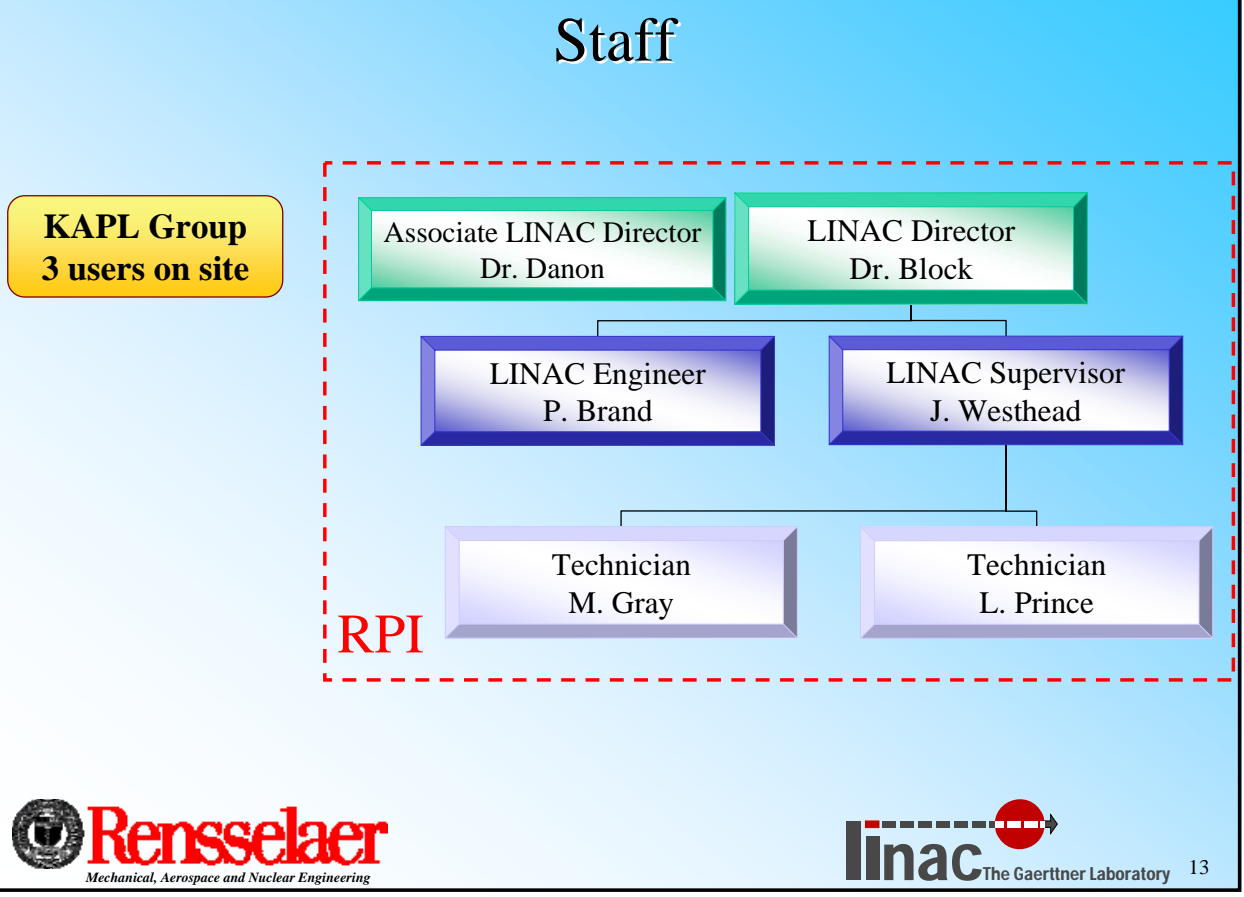

\section{Current Activity}

- Nuclear Data

- RPI-KAPL program

- Capture

- Transmission

- Capture to Fission ratio (alpha)

- New capabilities

- Scattering

- Transmission

- Upgrade the LINAC injector

- DOE-SSAA

- Simultaneous measurements of fission cross sections and fission fragment mass and energy distributions using the RPI lead slowing down spectrometer

- PXR generation

- Produced high intensity tunable X-rays

- Demonstrated imaging possibilities.

- Bubble fusion (NYSERDA/RPI)

- Service

- Radiation testing (JPL, SANDIA,ACTEL, Lockheed Martin, Ball Aerospace )

- Radioisotope production (Alpha Med, Harvard Medical School) 

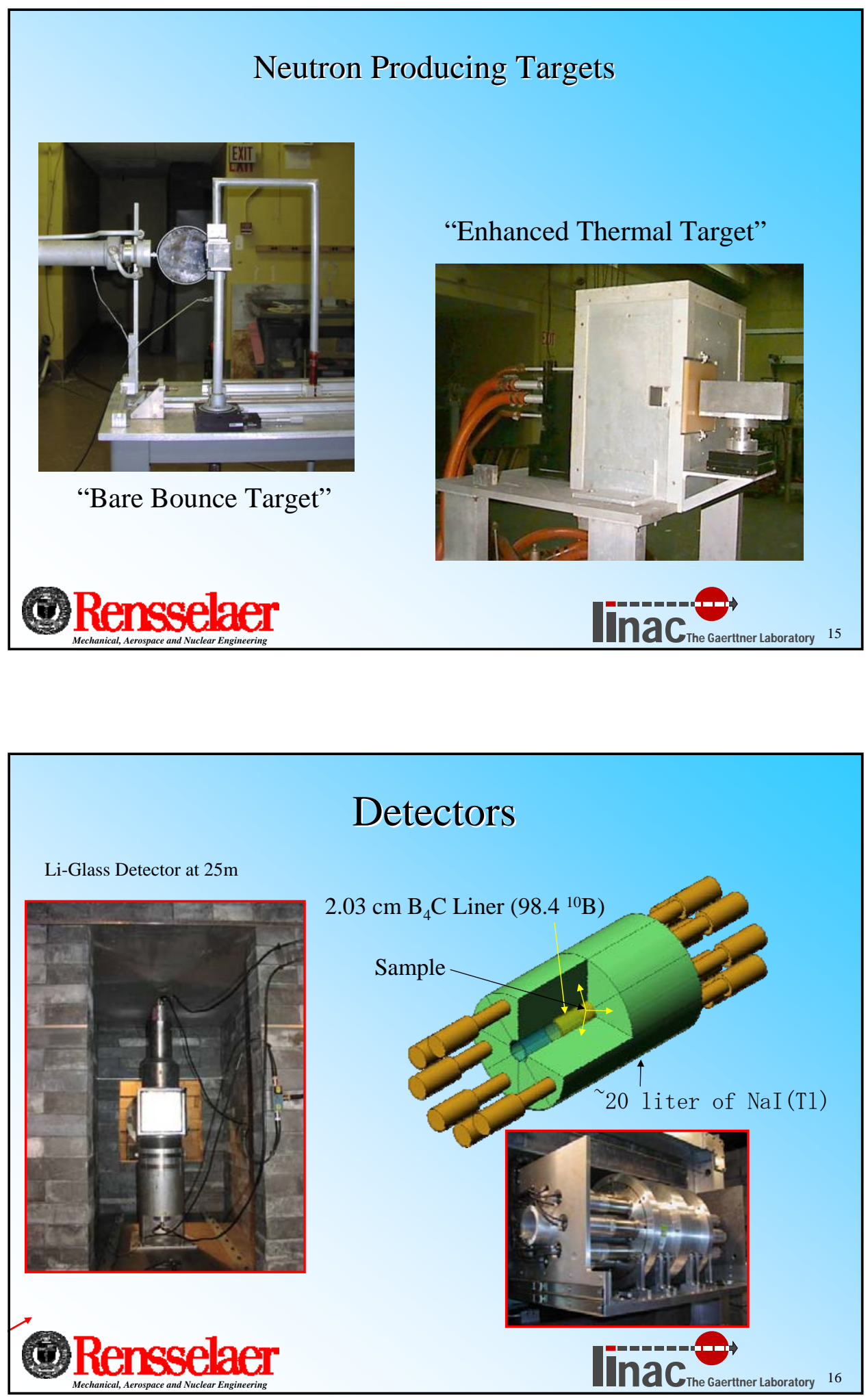

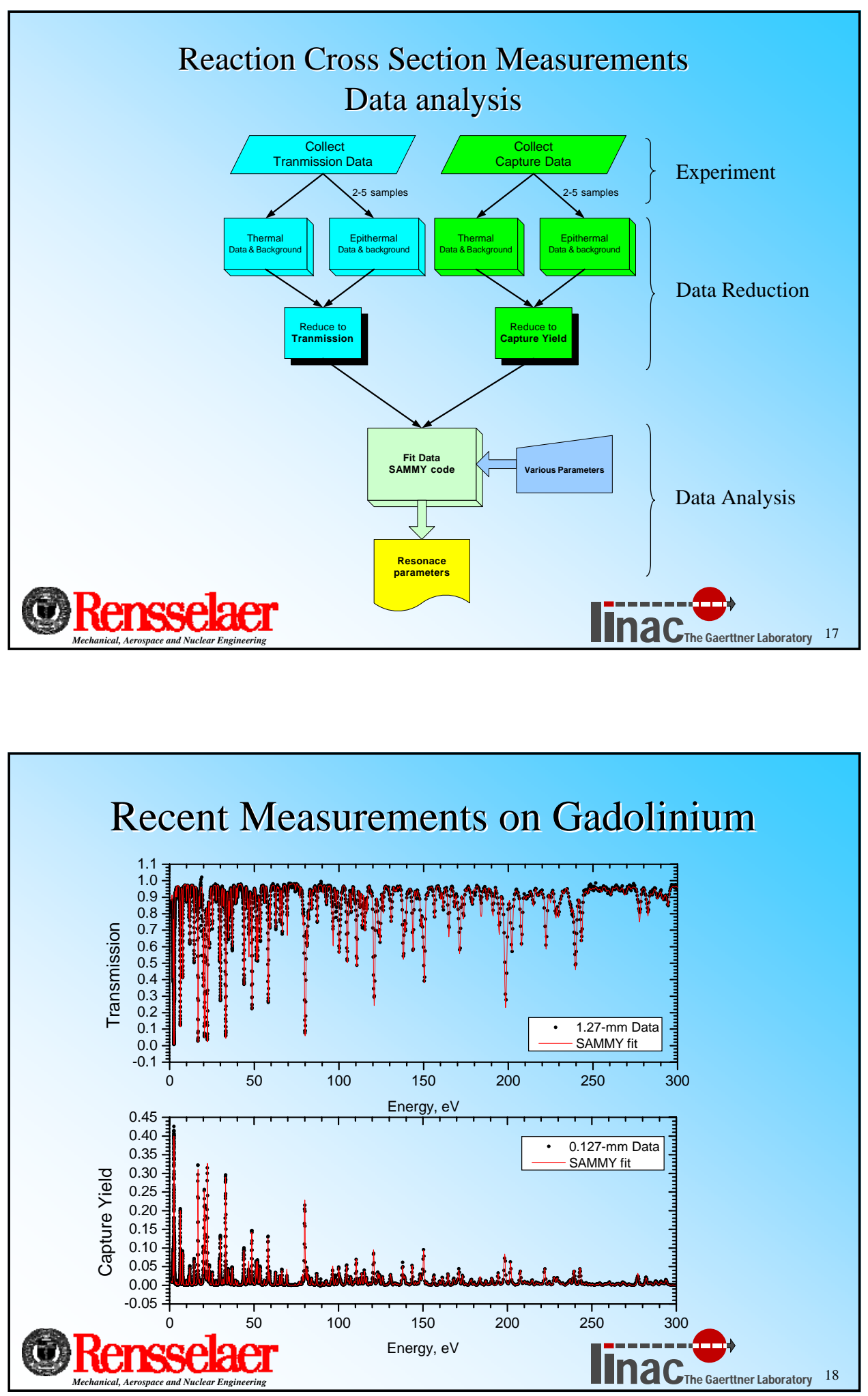

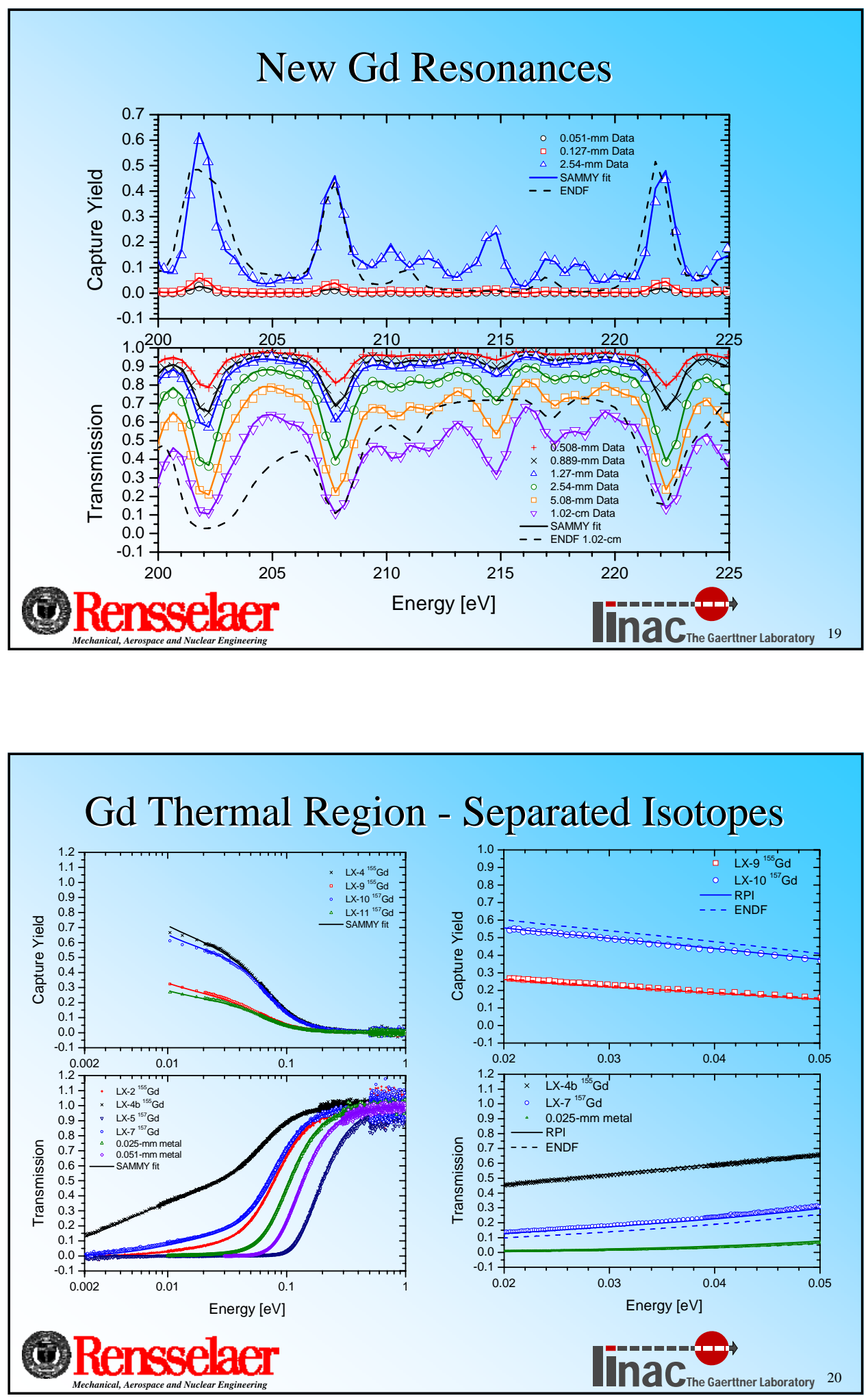


\section{In Process Development High Energy (0.2-10 MeV) Measurements}

- Build two new neutron detectors

- A modular detector at the $100 \mathrm{~m}$ station

- Higher energy transmission measurements

- Quasi integral scattering measurements

- Eight detectors and electronics for a differential scattering system.

- New injector system

- Provide short pulses (<10ns)

- Better beam focusing

- Spare parts

\section{ORensselaer}

\section{New Injector \& Gun}

- New $\sim \$ 0.75 \mathrm{M}$ gun-injector under construction

- ANL light source gun \& injector parts

- KAPL providing $\sim \$ 0.3 \mathrm{M}$
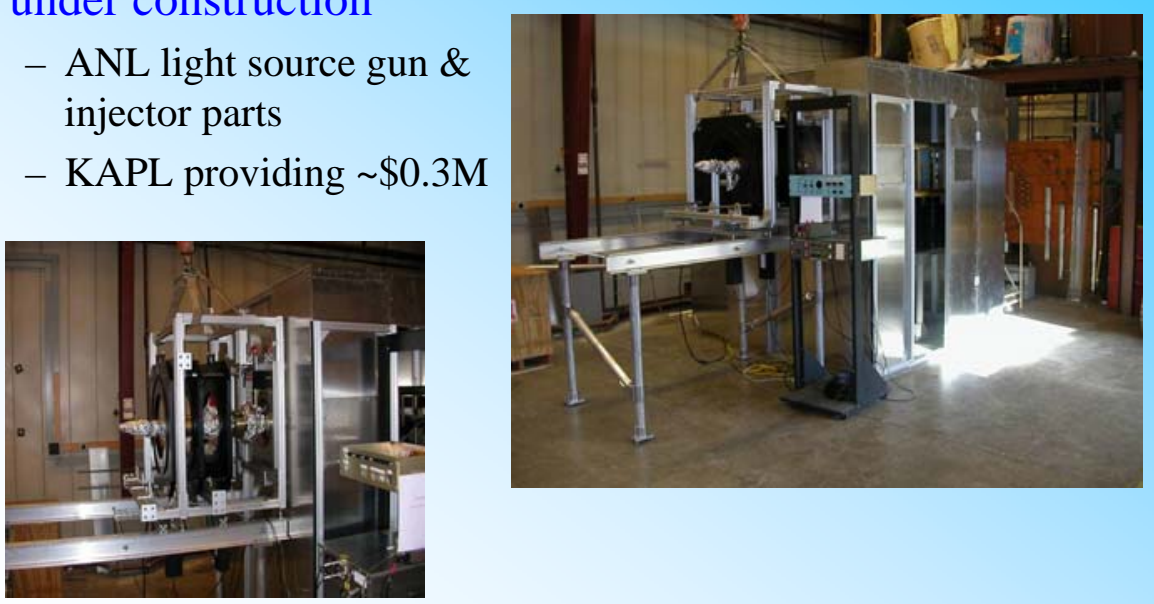


\section{Meter Modular Neutron Detectors}

- Extend the transmission measurements to higher energies (0.2-10 MeV).

- Perform quasi-integral measurements.

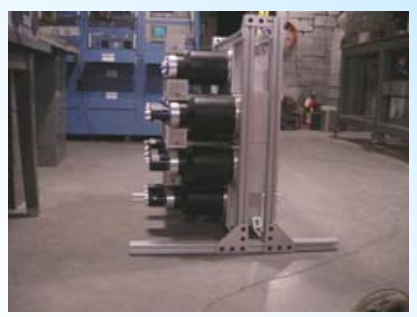

\section{ORensselaer}
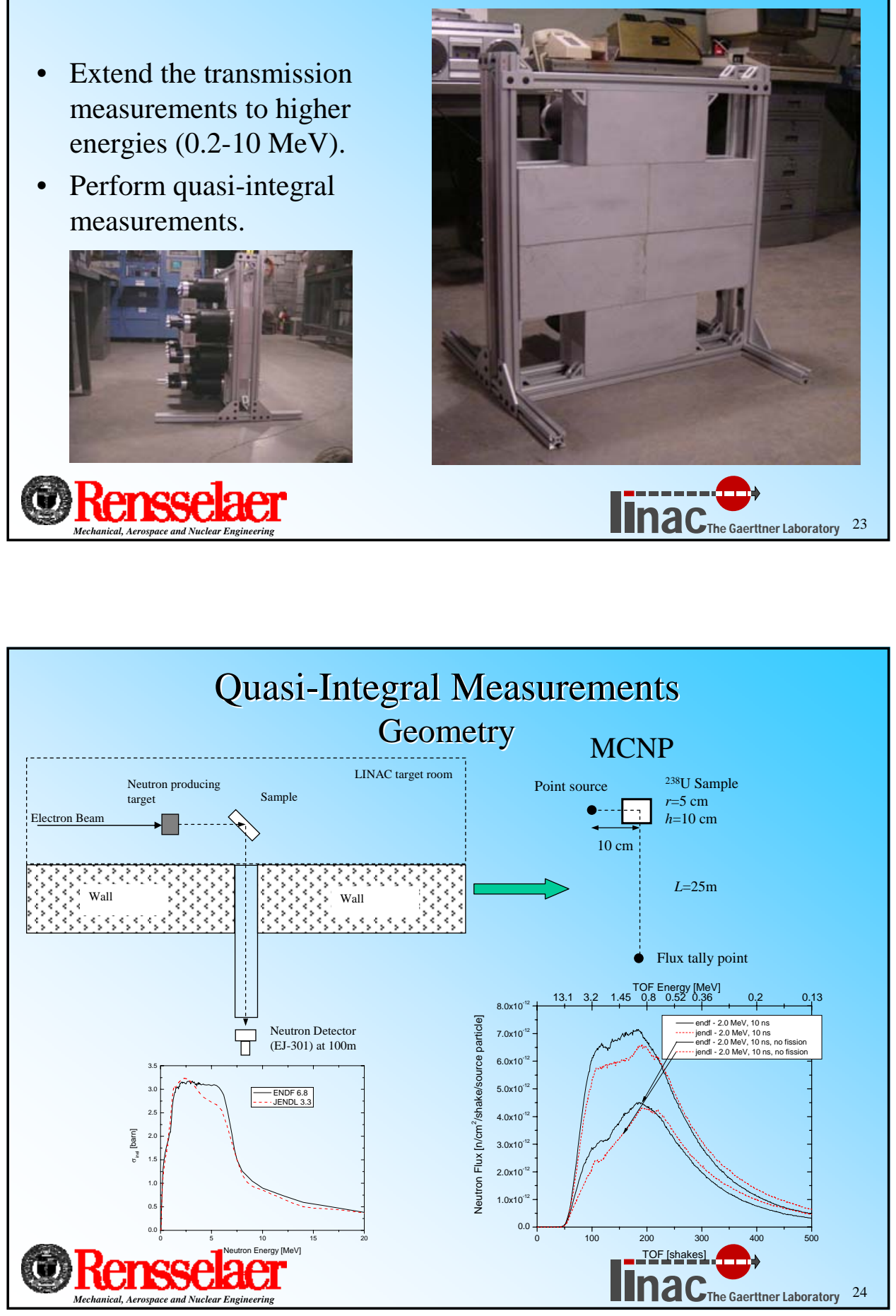


\section{Scattering Detector System (25 m)}

- Accurate measurements of neutron scattering as a function of energy and scattering angle.

- Energy range 0.3-10 MeV
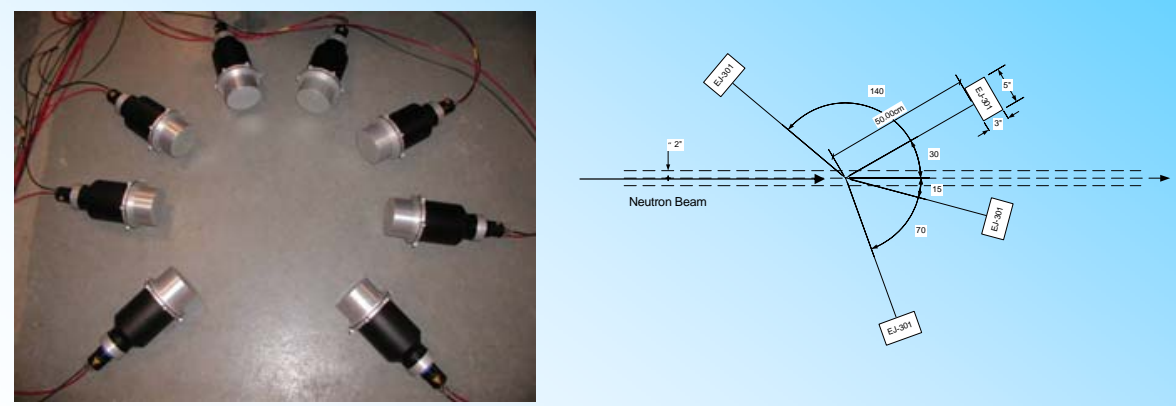

\section{ORensselaer}

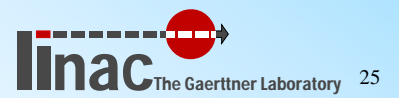

\section{${ }^{235}$ U Alpha Measurements}

- Measurements with ${ }^{235} \mathrm{U}$ Samples

- Multiplicity detector

- Looked above and below neutron binding energy

- Need for a ${ }^{235} \mathrm{U}$ Fission Chamber

- Determine detector response for ${ }^{235} \mathrm{U}$ resonances

- Developing a Solar Cell Fission Detector 


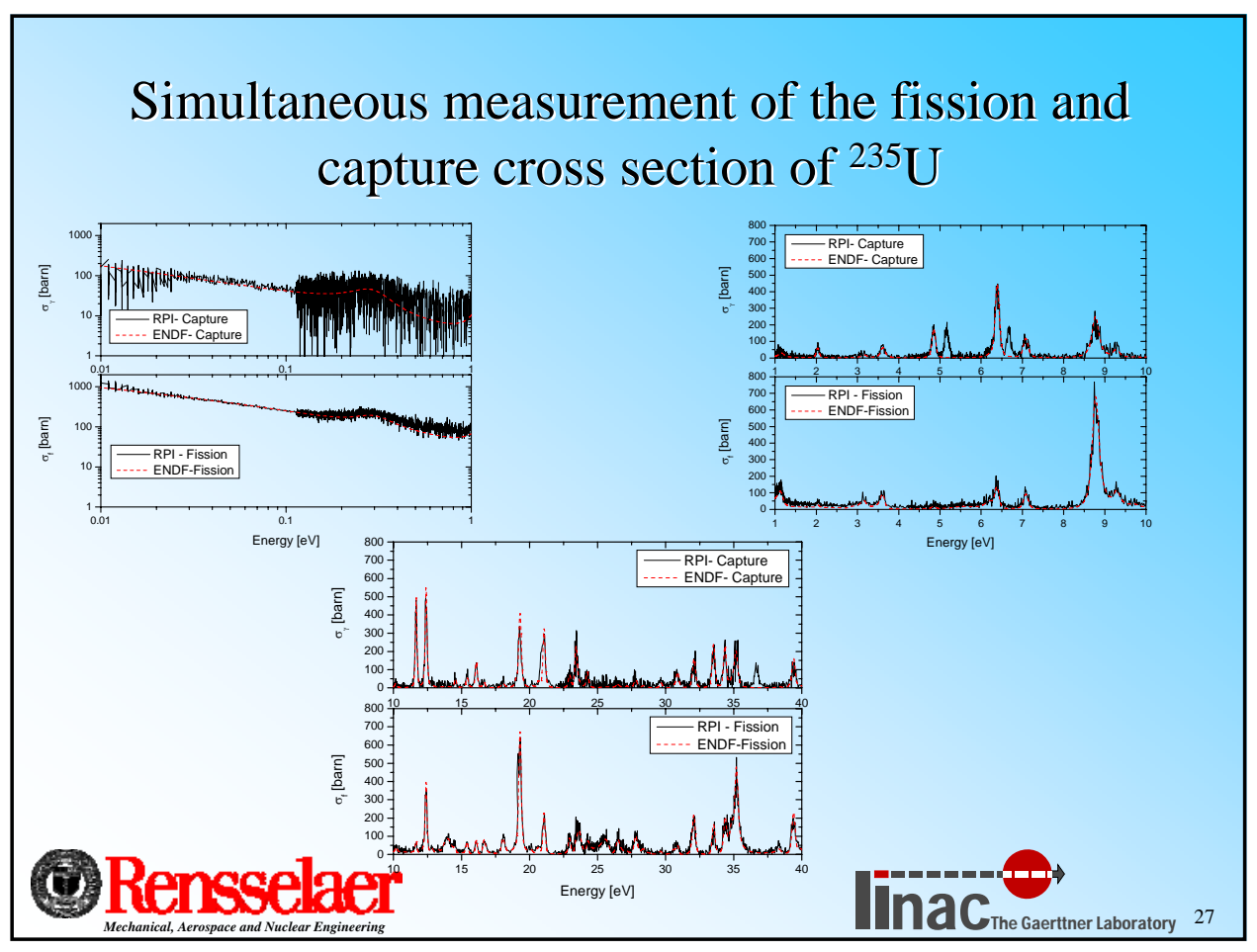

Fission Fragment Kinematics

- Objective

- Measurement of the fission cross section and fission fragment mass and energy distributions of small samples ( nanograms).

- Use the RPI lead slowing down spectrometer and a double gridded fission chamber.

- Samples are deposited on very thin backing (120 nm thick polyimide with $15 \mathrm{~nm}$ gold coating) 


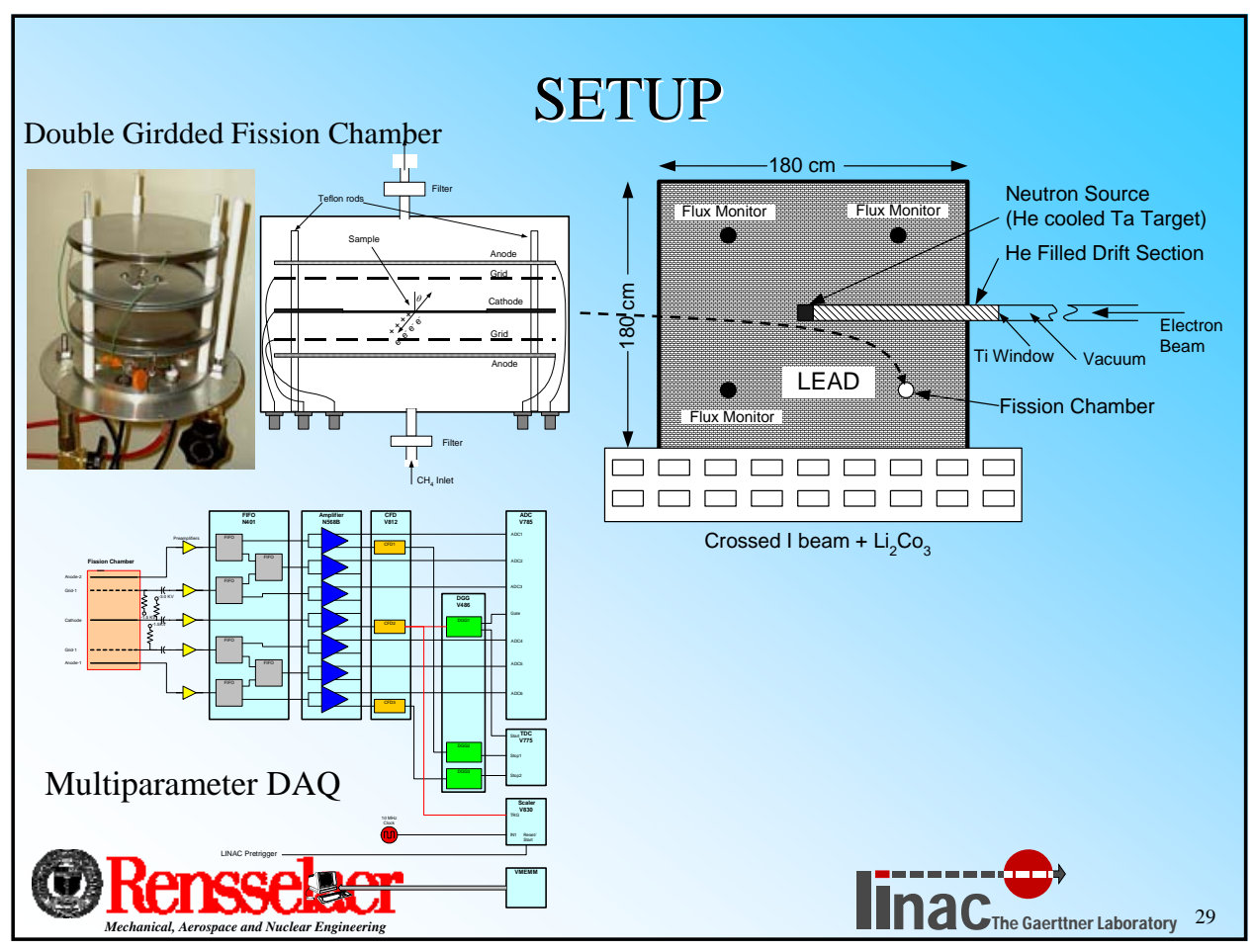

Test Data for a ${ }^{252} \mathrm{Cf}$ source on thick backing

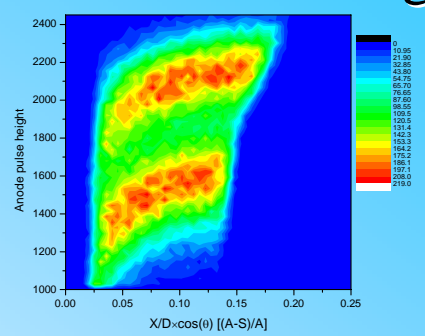

-... Anode
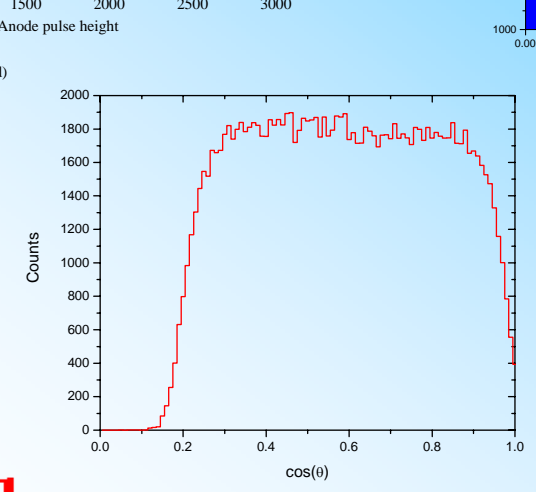

Mechanical, Aerospace and Nuclear Engineering 


\section{5 \\ The LINAC in Education}

- Use the LINAC in courses

- INEEP

- Photon activation experiment

- NEEP

- Neutron/Photon activation analysis

- Total cross section measurements

- Related courses

- Radiation Technology - Undergraduate Level

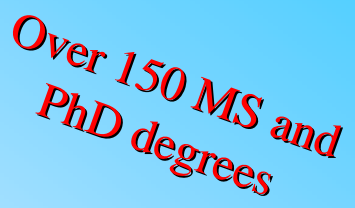

- Radiation Applications - Graduate Level

- Special Topics - Fission Physics - Graduate Level

- Senior design projects

- Positron production target (best project award)

- Production of medical isotopes

- Imaging using PXR (best project award)

- Electron beam position monitor.

- URP projects

- Low current (nA) beam monitor.

- Positron lifetime spectroscopy.

- Fission fragment spectroscopy.

- Positron annihilation Imaging

\section{ORensselaer}

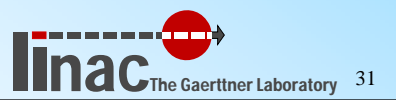

\section{Conclusions}

- Upgrades

- The RPI LINAC undergone a major ( $\sim 1.4$ million) completed in 2000

- The injector system is currently being upgraded

- Various other components upgrades (pulse transformers, thyratrons)

- Capabilities

- RPI houses a unique lead slowing down spectrometer and a multiplicity detector

- Measurements of high precision total and capture cross sections in the resonance region

- Established data reduction and analysis procedures (production mode).

- New capabilities

- Transmission and scattering measurements in the energy range from 0.2 $\mathrm{MeV}$ to $10 \mathrm{MeV}$.

- New Transmission detector located at the $100 \mathrm{~m}$ station

- New scattering detectors and DAQ located at the $25 \mathrm{~m}$ station 


\section{The Gaerttner LINAC Laboratory} Review and Current Activity

$$
\text { ORELA Workshop July 14-15, ORNL }
$$

Y. Danon and R.C. Block

Rensselaer Polytechnic Institute, Troy, NY, 12180

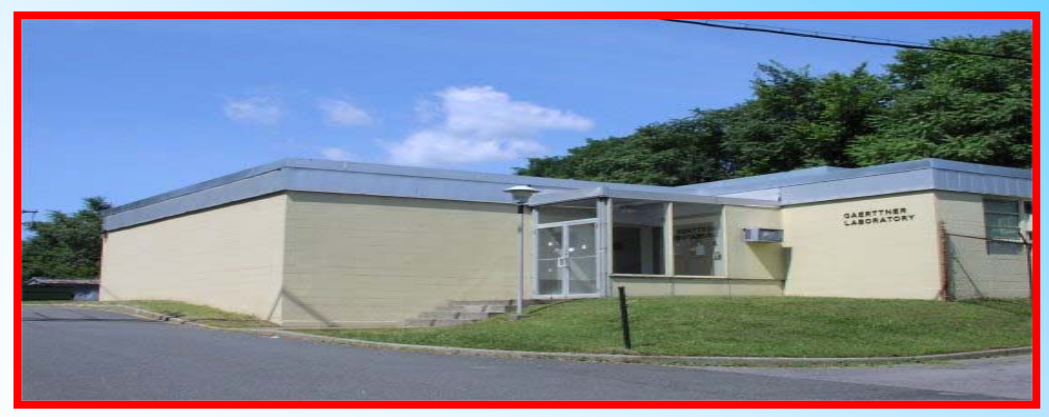

\section{(0) Rensselaer}

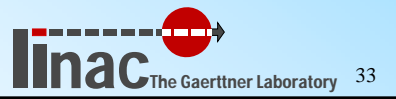

\section{Backup Slides}

\section{ORensselaer}

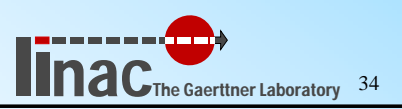




\section{New Injector and Gun}

- Required for High Energy Measurements

- High peak currents from electron gun

- Narrow electron pulse widths

- Existing Injector and Gun

- Limited peak current

- Relatively wide electron pulses

- Only adequate for thermal to low keV measurements

- Gun no longer manufactured or refurbished

- Need a new injector and gun

\section{Argonne Gun}

- Dr. Koontz Consulting Visit: January $12^{\text {th }} \& 13^{\text {th }}, 2004$

- Electron Gun Evaluation, Injector Enclosure Design, and Project Planning

- Installed cathode/grid was unusable (this was a used assembly).

- Gun cathode/anode gap needs modification to get higher emission

- Gun body needs some minor internal cleaning and bakeout

- Result: RPI developed/constructed a gun bake-out system
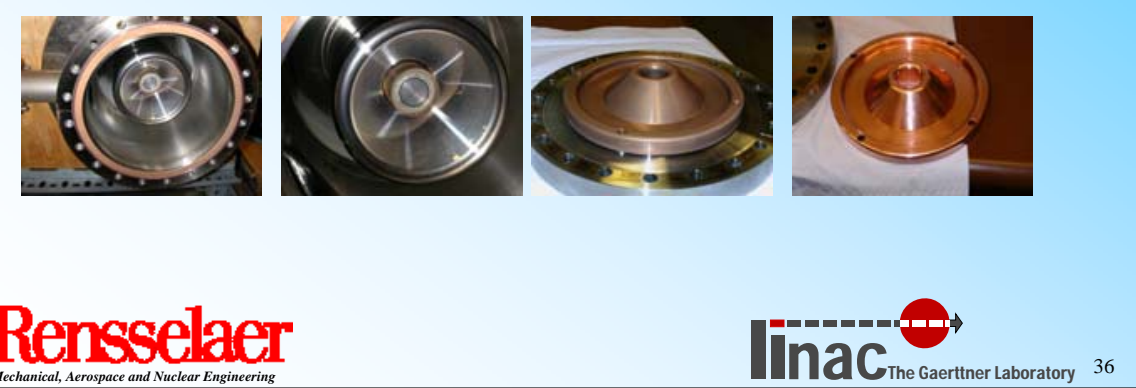


\section{Modeling of Injector \& Gun}

- Modeling of RPI LINAC: Peter Brand \& Rian Bahran (URP student)

- Accelerator Modeling (two different types of section used on RPI LINAC)

- First Section: Constant Gradient - 2П/3 (3 cavities/wavelength)

- Sections 2 - 9: Constant Impedance - I /2 (4 cavities/wavelength)

- Magnetic Field, Radio Frequency, and Beam Modeling Software

- Goals:

- Determine field parameters for our solenoidal focusing magnets and the overall injector assembly with magnetic lenses and helmholtz coils

- Determine the space harmonics for our accelerator sections

" This is a matrix or field plot that defines the vector accelerating potential within the aperture volume in the cavities of the accelerator section

- Create a model of our entire beam-line in PARMELA

" This utilizes the gun, magnetic lenses, and cavity modeling

- Assistance/Guidance from Roger Miller, Anatoly Krasnack, Ron Koontz, and Ron Aker at SLAC

- Result of Peter Brand's trip to SLAC in April '04

\section{ORensselaer}

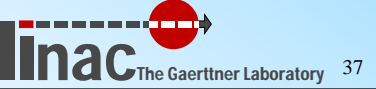

\section{New Injector \& Gun}

- Injector Test-Setup under construction
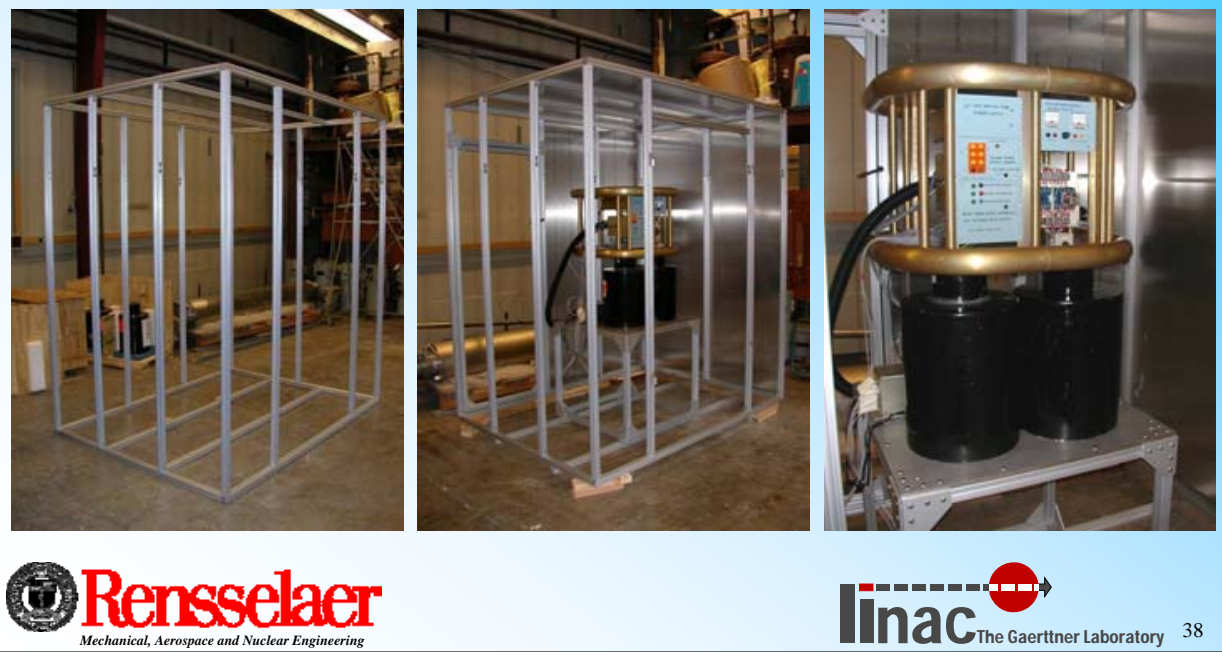


\section{Pulse Transformer: Replacement}

- New Pulse Transformer Manufactured by Stangenes Industries

- $\quad$ First Unit

- $\quad$ Ordered November 2002

- $\quad$ Delivered March 2003

- Installed and Tested in Modulator \#3 in April 2003

- $\quad$ Performed as expected (operation like original)

- Two Additional Units Received

Will be installed/tested in late summer/early fall

Seven More Units Needed to Complete All

Modulators and One Spare.

_ $\quad$ Cost : 2 @ \$11,600 each or $7 @ \$ 10,400$ each (plus shipping)

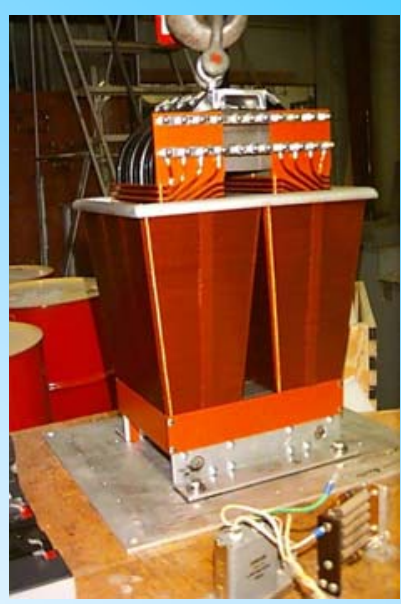

\section{ORensselaer}

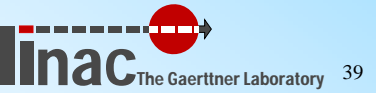

\section{Thyratrons}

- Thyratron in use now

- ITT Model KU-275

- No longer in production

- Last spare used in November of 2003

- Need one or more spares now

- Replace with L-4888

- Used by SLAC

- $\$ 15,000$ each

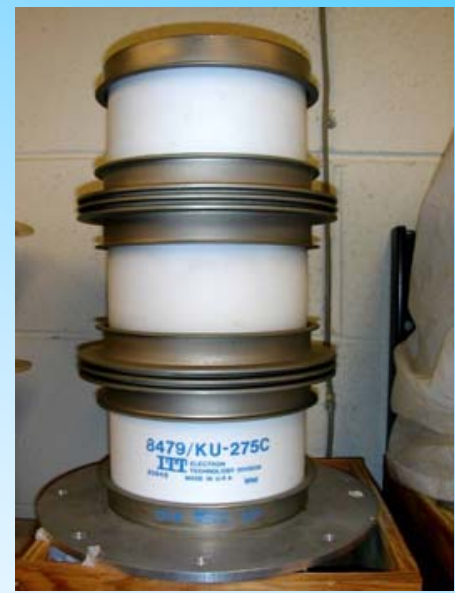




\title{
LANL - ORNL Nuclear Data Evaluation Collaborations for Supporting NCSP
}

\author{
M.B. Chadwick, R.C. Little, T. Kawano \\ Los Alamos National Laboratory
}

- Los Alamos

\section{Nuclear Data Evaluation for ENDF}

\section{ORNL/LANL Collaboration}

- ORNL : Resolved/Unresolved Resonance Regions

- ORELA experiments / SAMMY analysis

- ORNL resonance parameter evaluations

- LANL : Above Resonance Region

- LANL nuclear reaction modeling - GNASH/McGNASH

- Statistical Hauser-Feshbach model with pre-equilibrium emissions

- Combined Data

- Resonance parameters and cross-sections above the resonance region are combined and stored in the ENDF/B library.

- Benchmark Testing

- LOS Alamos

เม. $19 a 3$ 


\section{Unique Capability of ORELA for Neutron Capture}

Capture measurements at ORELA and data analysis at LANL for criticality safety study

- Experimental data in the unresolved resonance region

- The data for a natural element can be splitted into the isotope cross-section data by using the nuclear reaction theories.

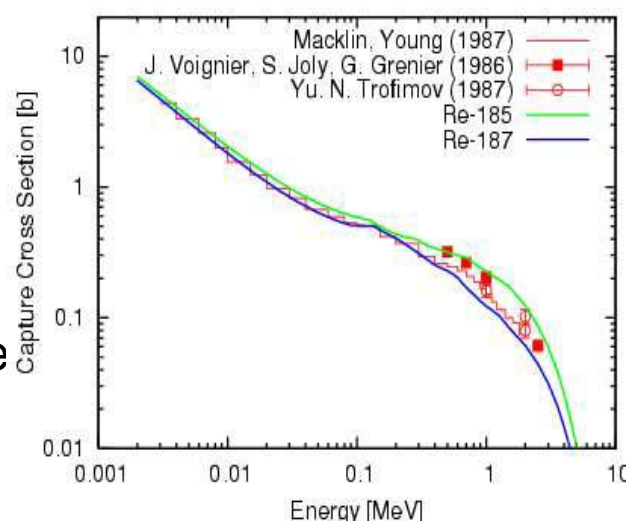

\section{Uncertainties in the Nuclear Data}

LANL-ORNL leads the covariance evaluations

- Resoance Region

- SAMMY analysis gives a covariance of resonance parameters

- Retroactive method for the existing nuclear data library

- Higher Energy Region

- Covariance evaluation using the KALMAN code

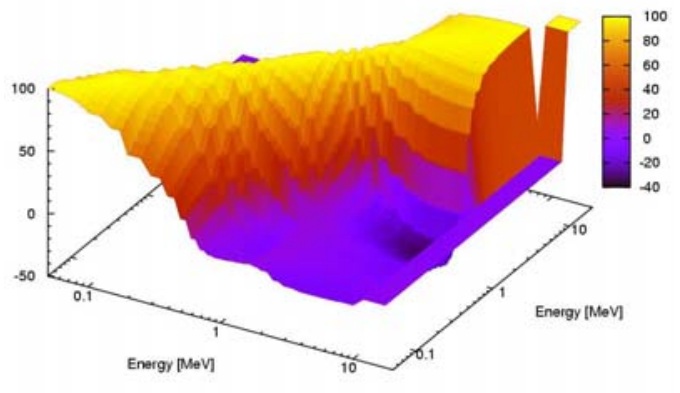

Correlation matrix for the capture cross section of $R e$

- Least-Squares method using the GLUCS and SOK codes

- LOSAlamos

เx.19sa 


\section{Covariance Evaluation for Gadolinium}

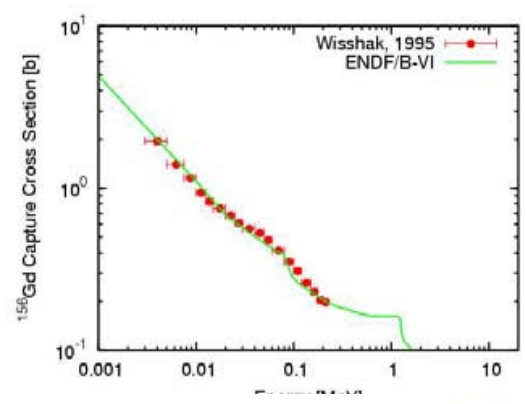

The KALMAN code was used to estimate the covariance of $\mathrm{Gd}$ capture cross sections in ENDF/B-VI.

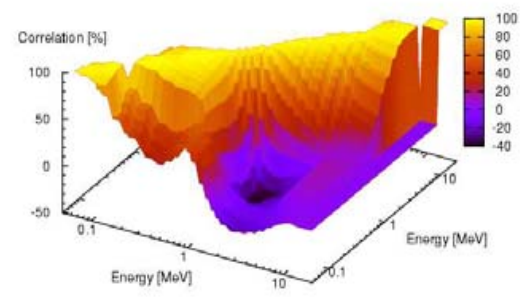

Correlation matrix for the capture cross section of Gd154

\section{New ENDF/B-VII Nuclear Data Library, Nov 2005. Actinides from Los Alamos evaluations}

- New ${ }^{232-241} \mathrm{U} ;{ }^{239} \mathrm{Pu} ;{ }^{237} \mathrm{~Np} ;{ }^{240-243} \mathrm{Am}$ evaluations

- Evaluation methods:

- experimental data where available

- nuclear theory predictions (GNASH, ECIS codes)

- systematic phenomenology of reactions on chains of isotopes (e.g. $\mathrm{n}+{ }^{237} \mathrm{U}$ requires fission barriers for ${ }^{238,237,236} \mathrm{U}$, but we have information on 238 barrier from $n+{ }^{238} \mathrm{U}$ second-chance fission data)

- $233,235,238 \mathrm{U} \longrightarrow$ strongly linked to experimental database

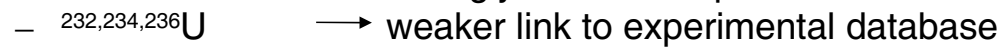

- $237,239,240,241 \mathrm{U} \longrightarrow$ based almost entirely on theory

- Integral data testing in critical assemblies, and pulsed spheres.

\section{- Los Alamos}

NATIONAL LABORATORY 


\section{Critical Assembly Integral Data Validation}

- In fast critical assemblies, we can model the assembly very accurately

- MCNP transport methods very accurate

- Geometries often very simple, e.g spheres, reflecting shells, etc.

- This allows us to:

- Validate nuclear data via comparisons against k-eff $(\operatorname{expt=1})$

- Test calculated integral reaction rates (fission, n2n, capture) within assemblies, since we know model the spectrum accurately

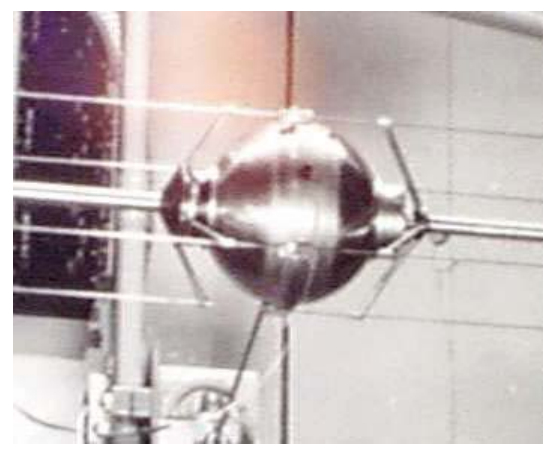

\section{Critical Assembly Integral Data Validation}

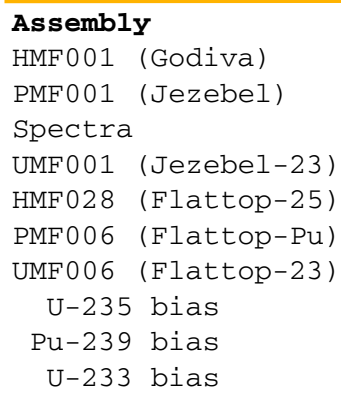

\section{- Los Alamos} Im.13as 


\section{Theory and Model Calculations}

- Coupled-channels optical model calculations with ECIS96 code

- Neutron transmission coefficients

- Total cross sections, $(n, n)$ and $\left(n, n^{\prime}\right)$ angular distributions

- Hauser-Feshbach / statistical / preequilibrium theory calculations with GNASH codes:

- (n, gamma), (n,2n), (n,3n), (n,4n), (n,n'continuum) cross sections

- Used with Kalbach systematics for continuum correlated energyangle distributions

- Los Alamos model used to calculate neutron emission spectra from fission of ${ }^{235} \mathrm{U}$ and ${ }^{238} \mathrm{U}$

\section{Optical Model Calculations}

- Used optical model potential developed at Los Alamos for ENDF/B-VI (plus variations)

- Coupled lowest 3-7 ground-state rotational band states

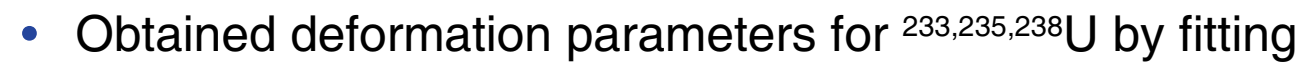
low-energy neutron scattering data

- Deformation parameters for other isotopes obtained by scaling $233,235,238 \mathrm{U}$ results using Moller-Nix calculations/compilation

- Performed extensive comparisons with calculations from other potentials by Vladuca et al., Maslov et al., Ignatyuk et al., and Sukhovitskij et al. 
Comparison of Various OM Potentials and Exp. $\sigma_{\text {tot }}$ Data

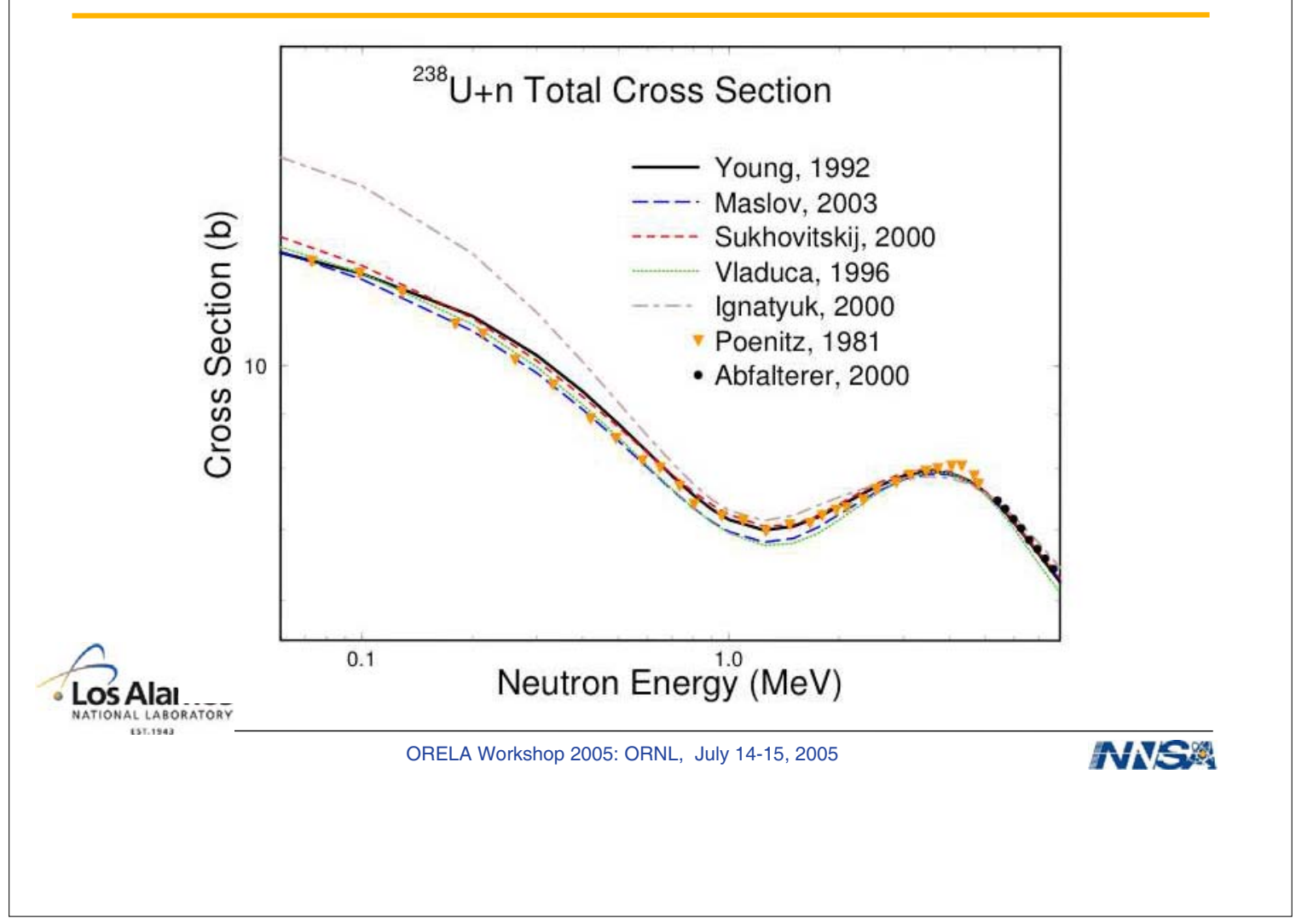

\section{Comparison of ${ }^{238} \mathrm{U}+\mathrm{n}$ Elastic Scattering Angular Distributions Calculated with Various OM Potentials}
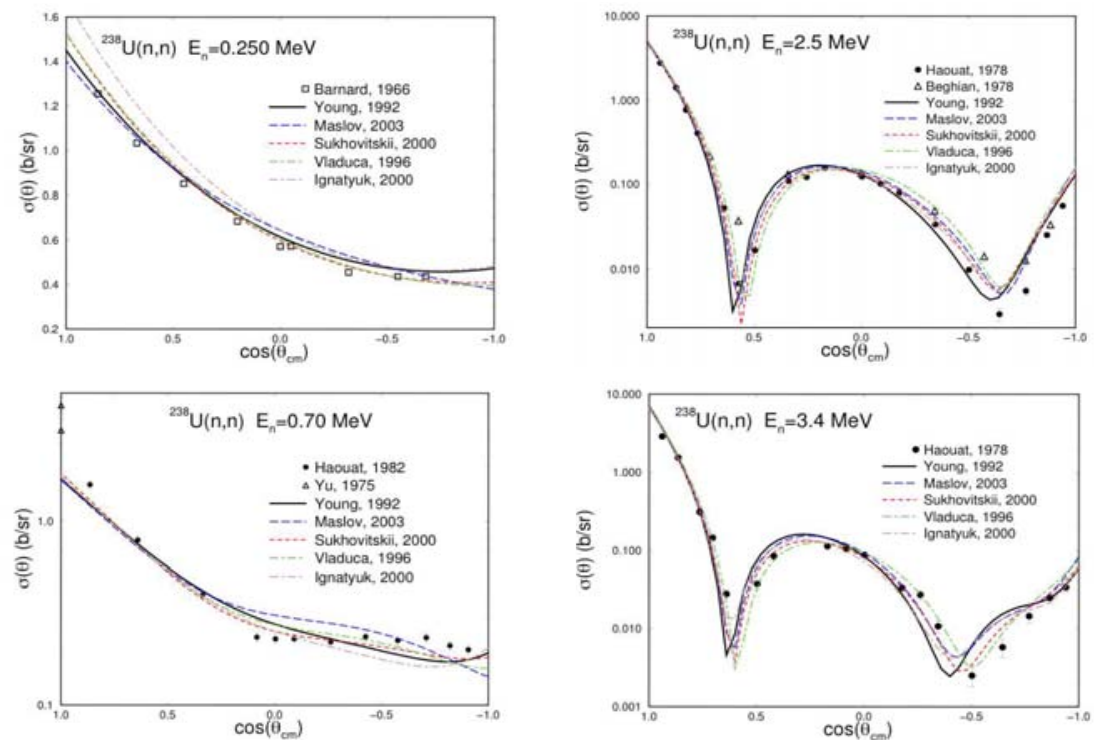

- Los Alamos 


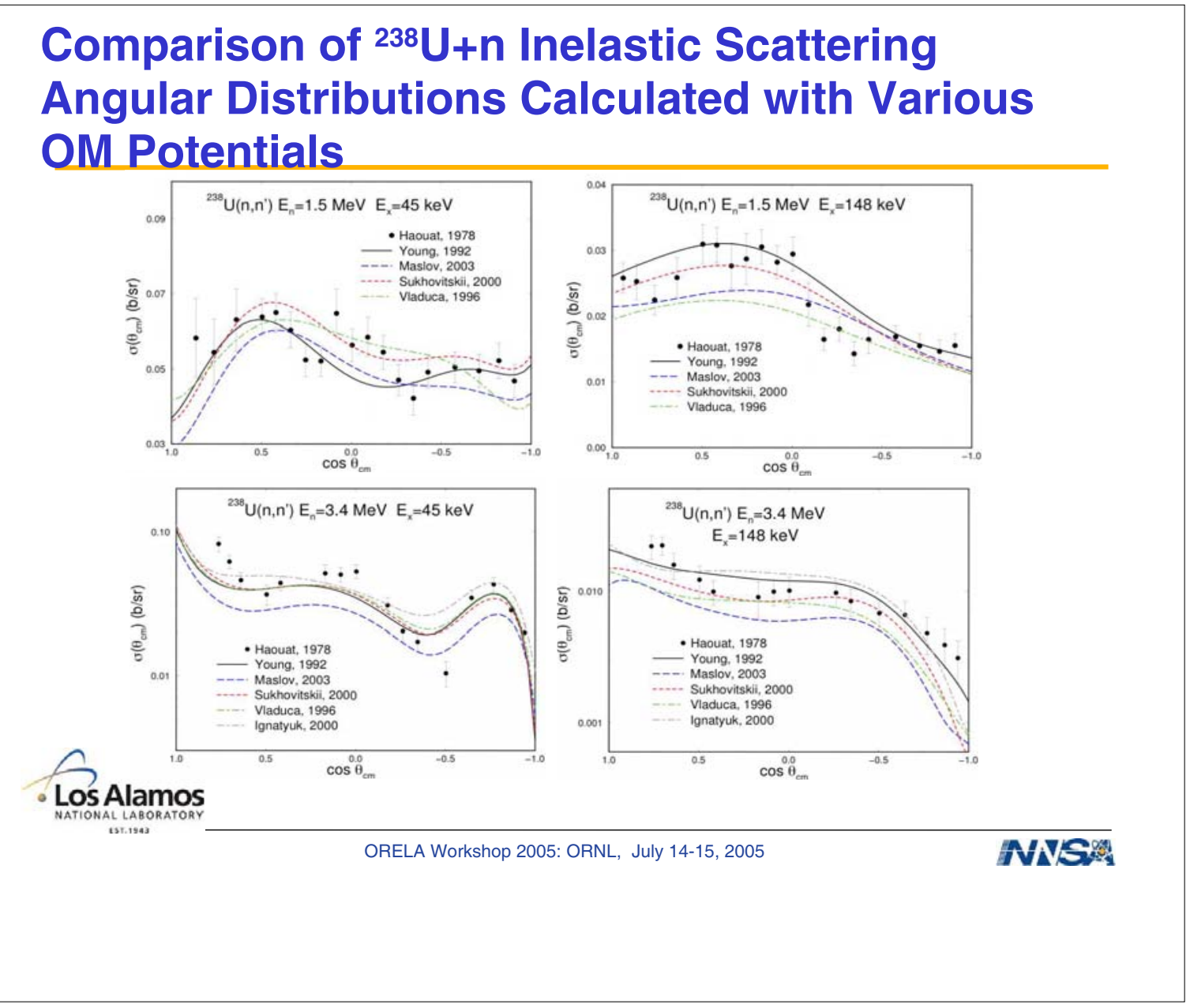

\section{Nuclear Data Standards (WPEC and IAEA)}

- Coordinated by A. Carlson and V. Pronyaev

- Re-evaluation / re-normalization of exp. data

- EDA R-matrix analysis for hydrogen

- R-matrix analysis for light elements

- Least-squares fitting for fission c.s.

- Combining two results
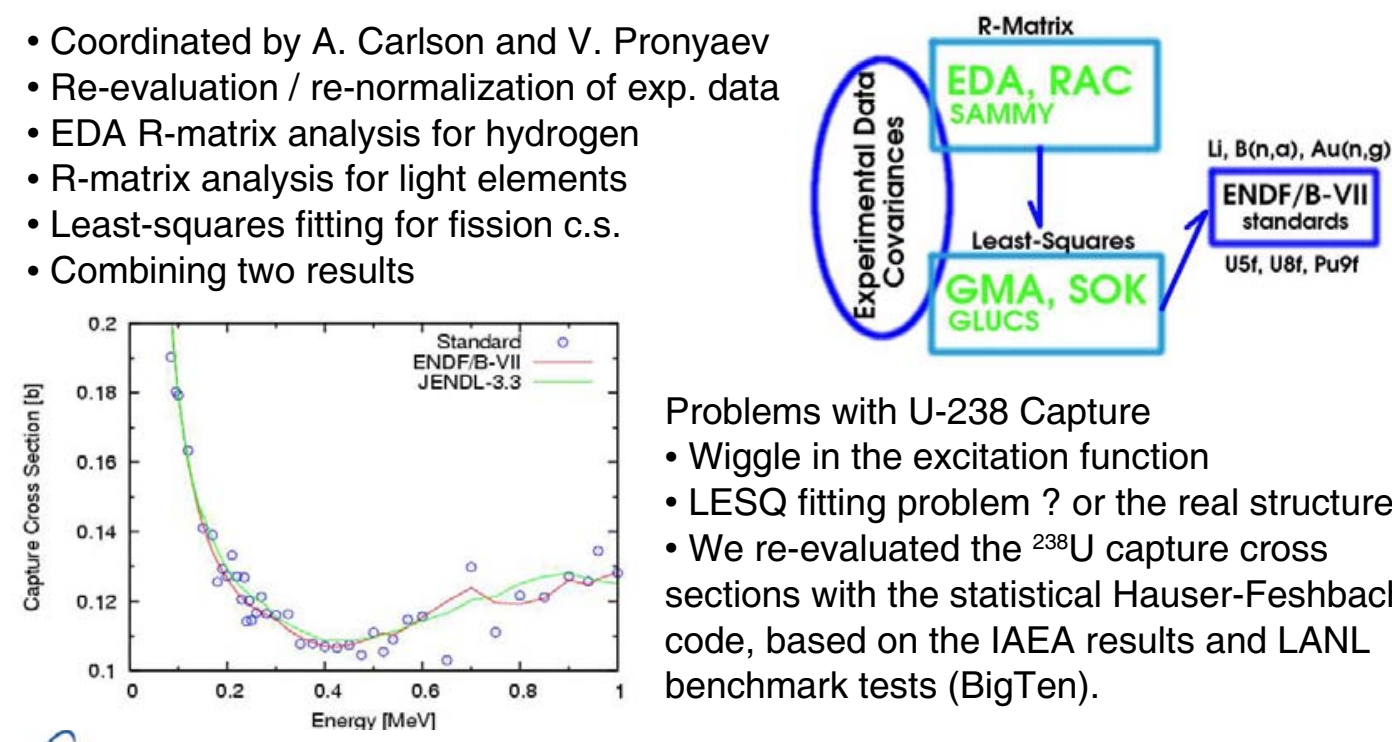

Problems with U-238 Capture

- Wiggle in the excitation function

- LESQ fitting problem ? or the real structure ?

- We re-evaluated the ${ }^{238} \mathrm{U}$ capture cross sections with the statistical Hauser-Feshbach code, based on the IAEA results and LANL benchmark tests (BigTen).

$\int_{\text {- LOSAONAL LABORATORY }}$ 


\section{Project Plans for FY05, FY06, and FY07}

- FY05

- Worked on ${ }^{9} \mathrm{Be}$ evaluation

- Problem reported for criticality with $\mathrm{Be}$ reflector. The total cross section was decreased to fix the problem.

- $\mathrm{Pb}$ evaluation

- Koning et al. made a new evaluation for JEFF-3.1, with careful modeling for the direct process.

- The elastic / inelastic scattering cross sections evaluated by Koning can be

- FY06 adopted

- ${ }^{235} \mathrm{U}$ capture, Complete ${ }^{9} \mathrm{Be}$ with R-matrix

- $\mathrm{FY07}$

- Np, correlated fission spectra, ${ }^{235} \mathrm{U}$ inelastic scattering(prelim.)
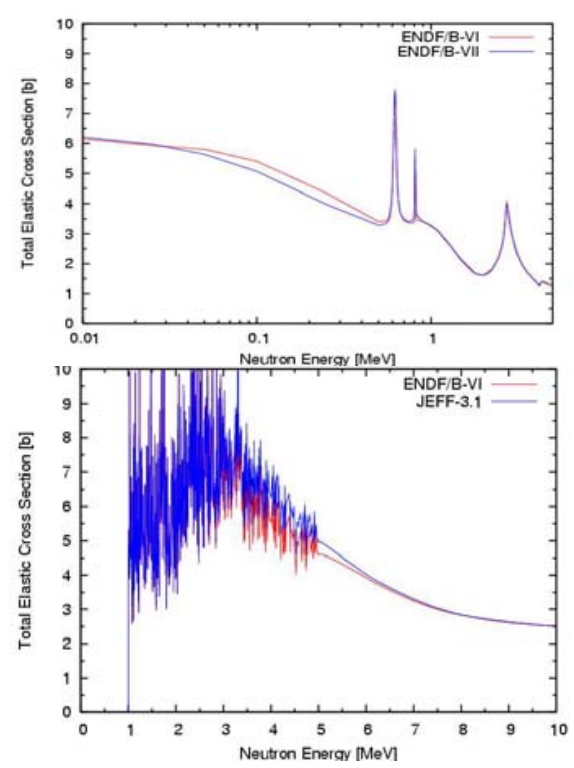

- Los Alamos

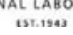

\section{Concluding Remarks}

- LANL - ORNL nuclear data evaluation collaborations

- Cross section evaluations

- ORNL : Resonance parameters

- LANL : High energy region

- Covariance evaluation

- ORNL : Resonance parameter covariance by SAMMY

- LANL : Cross section covariance by KALMAN / SOK

- Benchmark testing

- ORNL : Sentitivity analysis

- LANL : Criticality benchmarks

- LOSAlamos

15.194s 


\title{
Low-energy neutron total and capture cross sections at ORELA and nuclear reaction models
}

\author{
Frank S. Dietrich
}

ORELA Workshop

Oak Ridge, July 14-15, 2005

This work was carried out under the auspices of the U.S. Department of Energy by the University of California, Lawrence Livermore National Laboratory under contract No. W-7405-Eng-48.

N-Division / Physics and Applied Technologies

\section{Introduction}

Importance of low-energy region accessible at ORELA

- Physics still not well enough understood after $\sim 50$ years, but needed for applications

- Interplay between resonance structure and energy-averaged quantities

Optical model

Radiative capture

Doorway states

New high-precision data from ORELA can significantly improve understanding of these topics - recent data show this 


\section{$\mathrm{S}_{0}, \mathrm{~S}_{1}, \mathrm{R}$ - from resonances to the optical model}

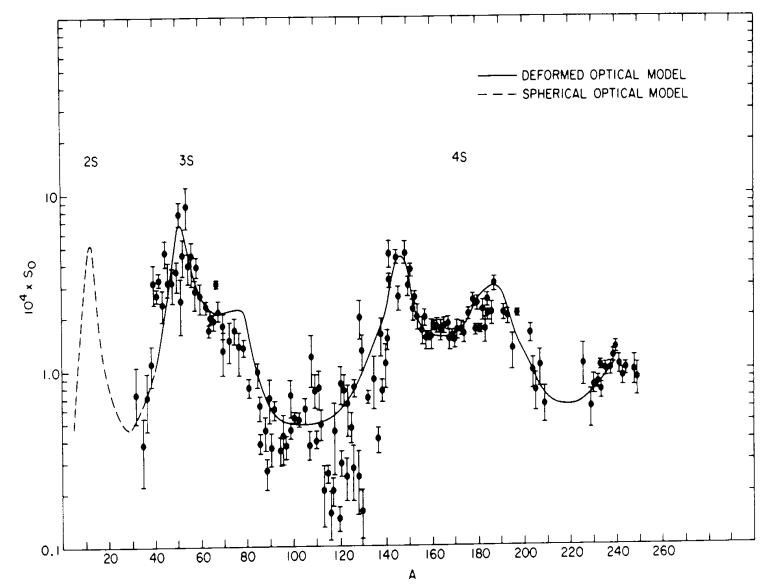

Measured strength functions $S_{0} \sim<\Gamma>/ D$ are optical model observables - why is there so much scatter?

Potential scattering radius $\mathrm{R}^{\prime}$ is also an optical model observable

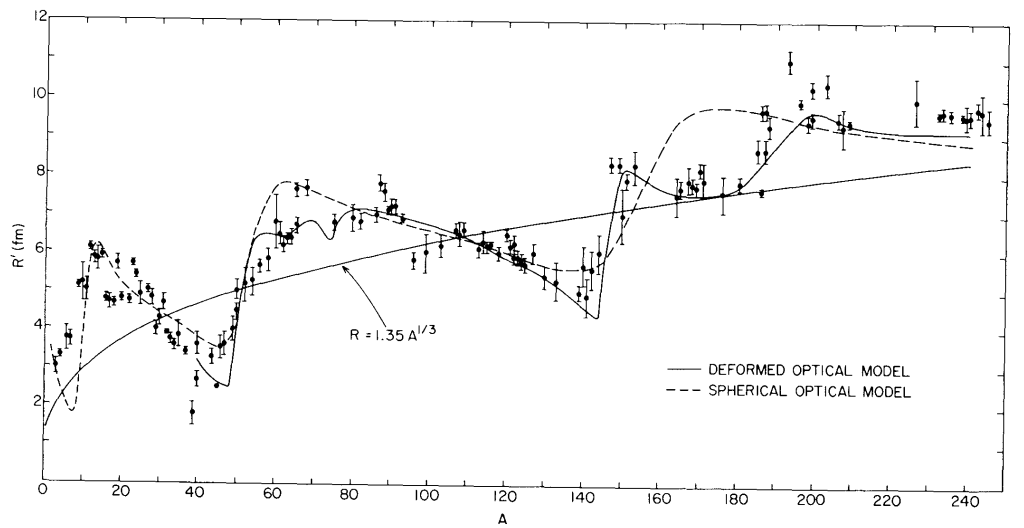

Determined mainly by interference between background and s-wave resonances

Significant deviations from optical model predictions 


\section{Intermediate structure ruins simple picture!}

Correspondence with smooth ( $E$ and $A$ ) optical model assumes slowlyvarying distributions of level spacings, partial widths

In many cases, experiment shows that this assumption is wrong

Models for intermediate structure (doorway states) were developed in the '60s (Feshbach, Kerman, Lemmer; Lane; many others)

Assumption: Path to compound nucleus goes through $2 \mathrm{p}-1 \mathrm{~h}$ states

Strength function becomes sum over doorways:

$$
\frac{\langle\Gamma\rangle}{D} \approx \frac{1}{2 \pi} \sum_{d} \frac{\Gamma_{d}^{\downarrow} \Gamma_{d}^{\uparrow}}{\left(E-E_{d}\right)^{2}+\frac{1}{4} \Gamma_{d}^{2}}
$$

\section{Examples of intermediate structure}

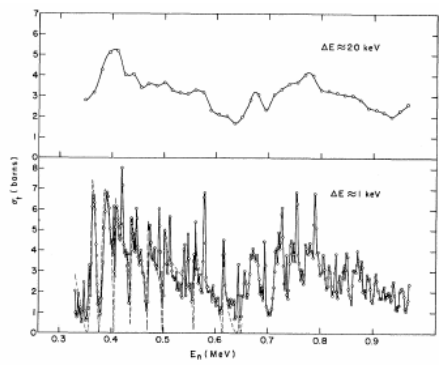

Neutron total cross sections on ${ }^{56} \mathrm{Fe}$ (Monahan/Elwyn)

Recent analysis of s-wave resonances for $\mathbf{n + 3 5 C l}$ (ORELA; R. O. Sayer)

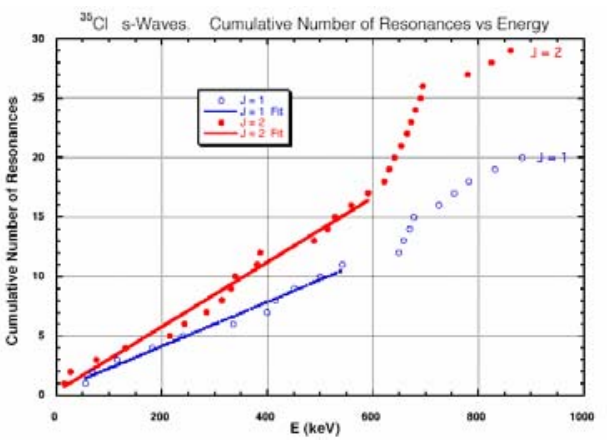




\section{Early calculations point in right direction}

- Shakin (1963) qualitatively reproduced some S0 results in the $\mathrm{Sn}$ region

- Initial state neutron couples to 3-quasiparticle doorway states in compound system

- Width of doorway states is a parameter

- How reliable are the experimental data?

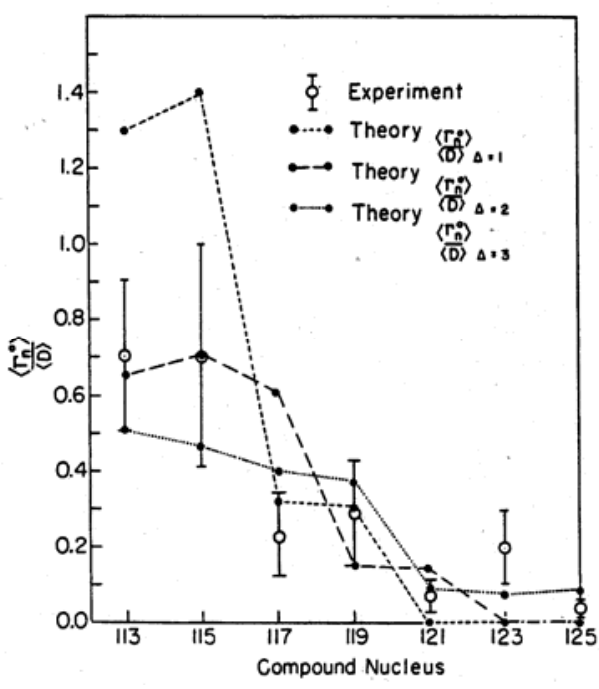

N-Division / Physics and Applied Technologies

\section{Challenges to experiment and theory}

Most experiments are now very old. Can ORELA significantly improve them?

- Better determination of strength functions

- Better characterization of anomalies in energy dependence of resonance parameters

Early doorway-state calculations used fairly crude structure models. Damping widths of the doorway configurations have not been calculated. Can modern shell model or HFIRPA treatments do better?

- Realistic treatment of damping (coupling to 3-particle 2-hole configurations)

- Adequate treatment of antisymmetry with incident neutron 
$(n, \alpha)$ reactions determine alpha optical potential (or do they?)

Alpha optical potential is important for astrophysics, but poorly known

$147 \mathrm{Sm}+\mathrm{n}$

Recent ORELA experiment measured alpha widths of resonances in an attempt to measure a strength function

Experiment showed a pronounced step in the cumulative $\alpha$ width distribution

How pervasive are these anomalies? Do they have a doorway-state explanation?

P.E. Kohler et al, PRC 2004
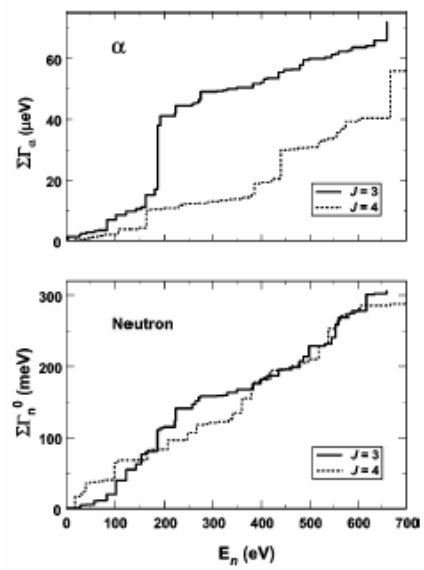

N-Division / Physics and Applied Technologies

Better $(n, \gamma)$ measurements needed to separate experimental and modeling uncertainties

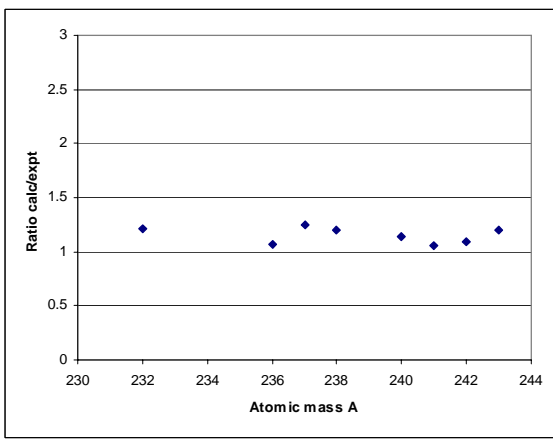

In actinides, simple $\mathrm{H}-\mathrm{F}$ model for capture using local systematics shows scatter of $\sim 10 \%$ for nonfissile targets

N-Division / Physics and Applied Technologies
Total $(n, \gamma)$ at $10 \mathrm{keV}$ on rare earths and actinides

In rare earths, similar calculation shows spread of $\sim 50 \%$

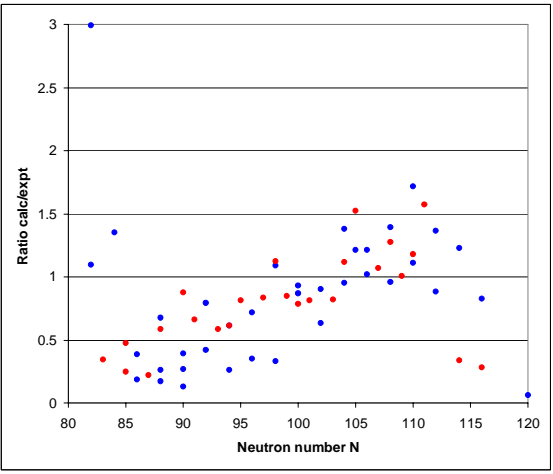




\section{Direct (and semidirect) radiative neutron capture}

Better understood at high (>1 MeV) than at low energies

Direct capture model needed at low energies for astrophysics, data evaluation

Theory ingredients not fully agreed on in background region between resonances

$$
\begin{aligned}
& \text { Lane/Lynn } \\
& \text { Lane/Mughabgab } \\
& \text { Cugnon/Mahaux }
\end{aligned}
$$

Measuring the background is difficult, has not yet been done. Can ORELA do it?

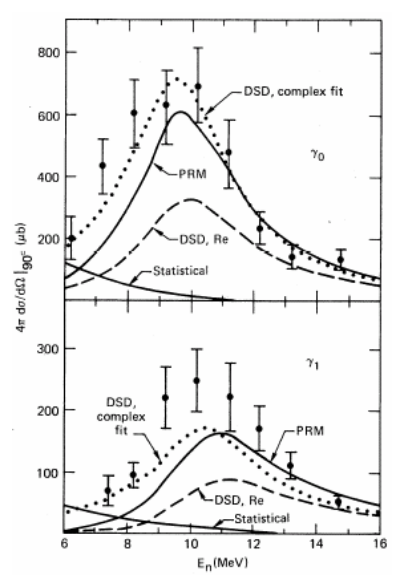

6 to $15 \mathrm{MeV}$ neutrons on ${ }^{208} \mathrm{~Pb}$

\section{Semidirect (GDR) term should not be neglected}

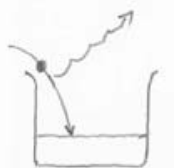

2 interfering terms in direct-semidirect capture:

Projectile radiates and is captured in well

DIRECT

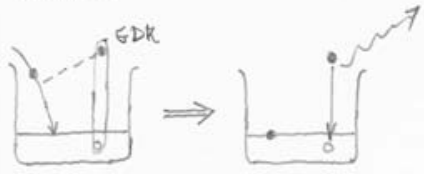

SEMIDIRECT

Semidirect term rarely used in low-E capture; it can be important

Effective radial electromagnetic operator:

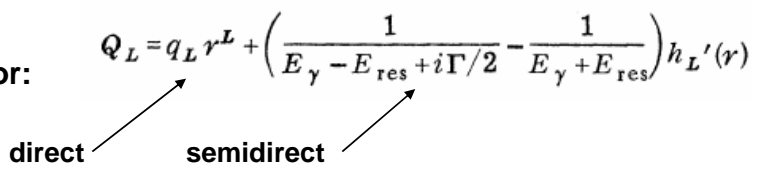

1) Projectile excites giant dipole resonance and is captured;

2) Giant dipole collapses and emits the gamma ray 


\section{Exact vs. approximate electromagnetic operator}

Interaction of nuclear system with electromagnetic field is

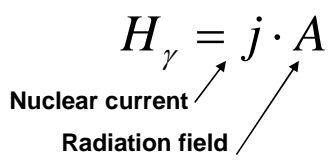

Standard approximation for electric multipoles: Use Siegert's theorem to replace nuclear current by nuclear density

Siegert's theorem is violated if initial and final state nuclear potentials are not identical. In fact, they are almost never identical

It requires very little extra work to use the exact (current) form in direct calculations; a good approximation is

$$
r^{L} \rightarrow r^{L}\left(1+\frac{V_{f}^{*}-V_{i}}{E_{\gamma}}\right)
$$

Using the exact form can be important - corrections up to factor of 2

N-Division / Physics and Applied Technologies

Thermal capture in s-d shell illustrates some of the problems

Direct capture calculations undertaken as part of evaluation of ORELA data

\begin{tabular}{|l|r|r|r|r|r|r|r|r|r|r|}
\hline & \multicolumn{1}{c|}{${ }^{19} \mathrm{~F}$} & ${ }^{27} \mathrm{Al}$ & ${ }^{28} \mathrm{Si}$ & ${ }^{29} \mathrm{Si}$ & ${ }^{30} \mathrm{Si}$ & ${ }^{35} \mathrm{Cl}$ & ${ }^{37} \mathrm{Cl}$ & ${ }^{39} \mathrm{~K}$ & ${ }^{41} \mathrm{~K}$ & $238 \mathrm{U}$ \\
\hline $\begin{array}{l}\text { Our } \\
\text { work }\end{array}$ & 6.5 & 60 & 151 & 111 & 98 & 430 & 418 & 799 & 544 & 2 \\
\hline TEDCA & ---- & ---- & 65 & & 67 & 160 & 310 & ---- & --- & ---- \\
\hline $\begin{array}{l}\text { Raman- } \\
\text { Lynn }\end{array}$ & 4.6 & ---- & 134 & 116 & 100 & ---- & ---- & ---- & --- & ---- \\
\hline $\begin{array}{l}\text { Lynn- } \\
\text { Lane }\end{array}$ & 4.7 & 108 & 107 & 70 & 64 & ---- & 400 & 753 & 1,320 & 80 \\
\hline Exp. ${ }^{\dagger}$ & 9.5 & 231 & 169 & 119 & 107 & 43,600 & 433 & 2,100 & 1,460 & 2,680 \\
\hline
\end{tabular}

* Real part of Koning-Delaroche opt. pot.; Error bars $\pm \sim 20-30 \%$

${ }^{\dagger}$ Error bars $\pm \sim 2 \%$

Limitations: s-wave only, resonant contribution not separable 


\section{Doorway states cause mischief to simple theory}

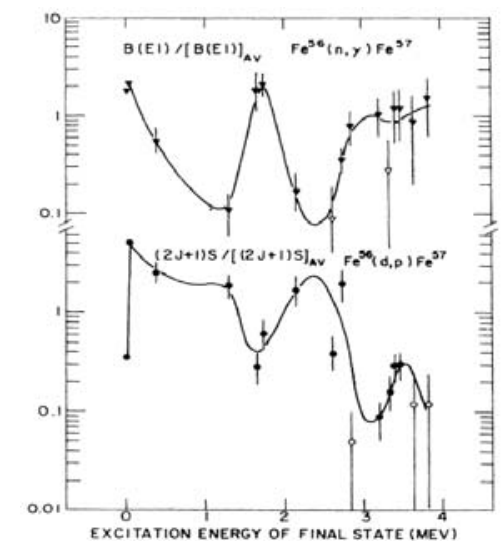

Example: ${ }^{56} \mathrm{Fe}(\mathrm{n}, \gamma)$ at thermal energy

Recall 56Fe+n showed evidence for intermediate structure

Direct capture csec should be proportional to $\mathrm{d}, \mathrm{p}$ spectroscopic factor

Correlation between $(n, \gamma)$ to specific final states and $(d, p)$ spectroscopic factors is disastrous

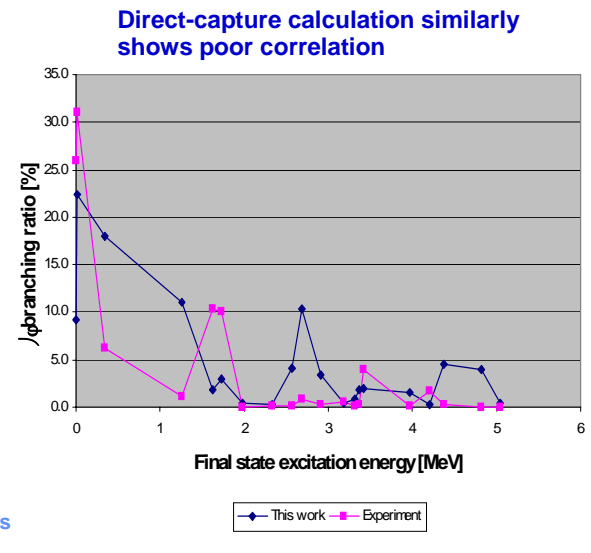

\section{Needed: good experimental values for capture between} resonances

Thermal results useful test, but subject to contamination from nearby resonances

Experimental backgrounds are hard to control, may mask the nonresonant direct component

Can adequate experiments be devised for a few good test cases?

- Subtract ORELA measurement of capture through resonances from activation measurement (Karlsruhe) for complete capture?

- Measure capture to specific final states with high-resolution detector? 


\section{Summary and conclusions}

Doorway states, 40 years old, need to be taken seriously

- Fundamental limitation on validity of optical model at low energies

- Fundamental limitation on ability to extrapolate off valley of stability

- Both neutron scattering and radiative capture affected

Reliable (total) radiative capture cross sections hard to find

- Often find either 1 measurement or several discrepant ones

Direct capture models need to be tested between resonances

A carefully focused program of precision measurements at ORELA is needed to address these issues

N-Division / Physics and Applied Technologies 



\title{
Nuclear Cross Sections for Stockpile Stewardship Applications at Livermore
}

\author{
Presented to: \\ ORELA Workshop \\ Oak Ridge National Laboratory

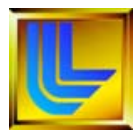

M. G. Mustafa

AX Division

Lawrence Livermore National laboratory

July 14, 2005

This work was performed under the auspices of the U.S. Department of Energy by the Univ ersity of Califormia Law rence Liv ermore National Laboratory under Contract No. W-7405-Eng-48.

Law rence Liv ermore National Laboratory, P.O. Box 808, Liv emore, CA 94551-0808

\section{Outline}

- Introductory remarks

- Nuclear data needs at Livermore

- Radiochemical Diagnostics

- Actinide cross sections

- Lighter masses $(A<10)$

- Measurements to assist in modeling

- Accuracy Accuracy Accuracy Accuracy

- Cross sections for stable nuclei

- Cross sections for unstable targets and isomers

- Fission product data

Accurate nuclear data and cross sections are central to Stockpile Stewardship. 


\section{Radiochemical Diagnostics}

- 23+ detectors (only 4 are reliable)

- Current and proposed experiments

- (n,gamma) measurements

- Total cross section measurements

- Isomer measurements

- Fission product measurements

- Surrogate measurements

- Extensive modeling and evaluations

We need cross-section data on stable, unstable and isomeric targets.

ORELA WORKSHOP - 3

\section{Uncertainties of measured and modeled} cross sections

- $(n, x n)$ reactions: 3-7\% (measured); 10-15\% (modeled)

- (n,gamma) reactions: 10-30\% (measured); 30-50\% (modeled)

- $(p, x n)$ reactions: 3-7\% (measured); 10-25\% (modeled)

- $(d, x n)$ reactions: 3-7\% (measured); 15-30\% (modeled)

Uncertainty reduction and quantification is the job. 


\section{Radiochemistry Needs (some specifics)}

- Capture cross sections in the europium and lutetium regions. (151Eu, 153Eu, 151Gd, 175Lu)

- Total cross sections (keV range) around mass 50. (48Ti, 48V)

- Nuclear data and cross sections on unstable targets and isomeric states (radiochemical detectors). (88Y, $89 \mathrm{Zr}, 170 \mathrm{Tm}, 171 \mathrm{Tm}, 173 \mathrm{Lu}$, 174Lu)

- More accurate fission and capture cross sections of actinides (uranium, plutonium, americium, neptunium).

\section{Radiochemical detectors: Sm-Eu-Gd}

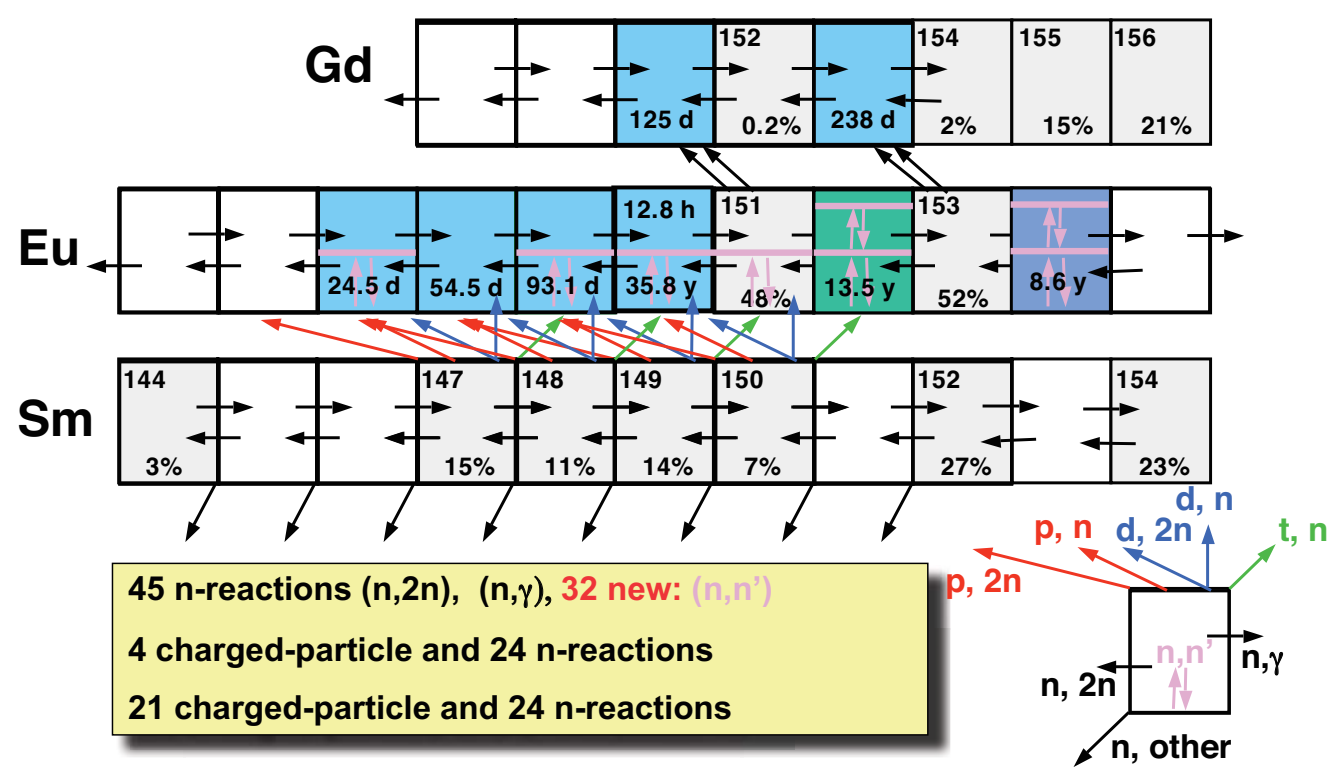




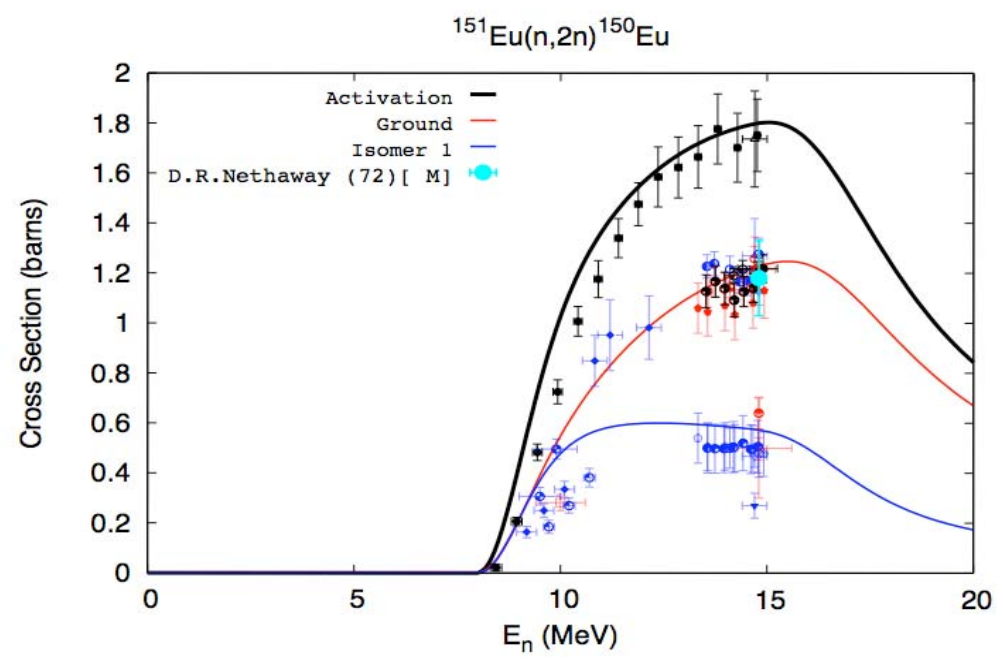

ORELA WORKSHOP - 7

\section{Large scattering in the (n,gamma) measurements.} Modeling has broad range.

\section{experiment}

Kononov 1977

Macklin 1987

Yiiun 1994 (exp)

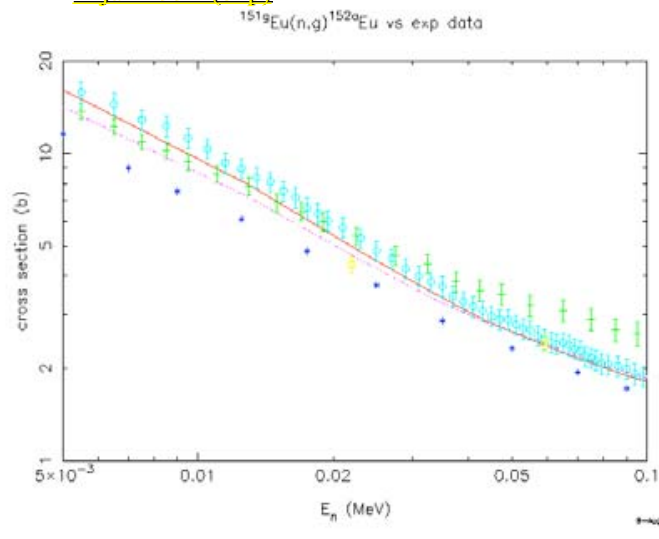

151Eu(n,gamma)152Eu calculations

Vibrational OM

Rotational OM

Rom

\section{Eu(n,2n) 150}


${ }^{151} \mathrm{Gd}(\mathrm{n}, \mathrm{gamma}){ }^{152} \mathrm{Gd}$ modeled cross sections and ratios

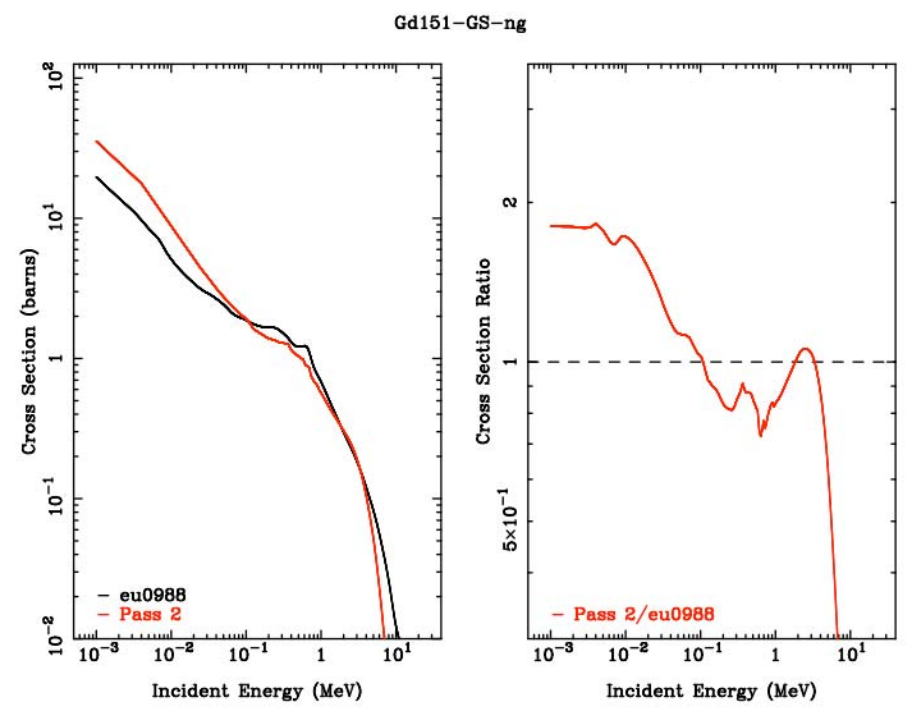

ORELA WORKSHOP - 9

Titanium-Vanadium (Ti-V) reaction network

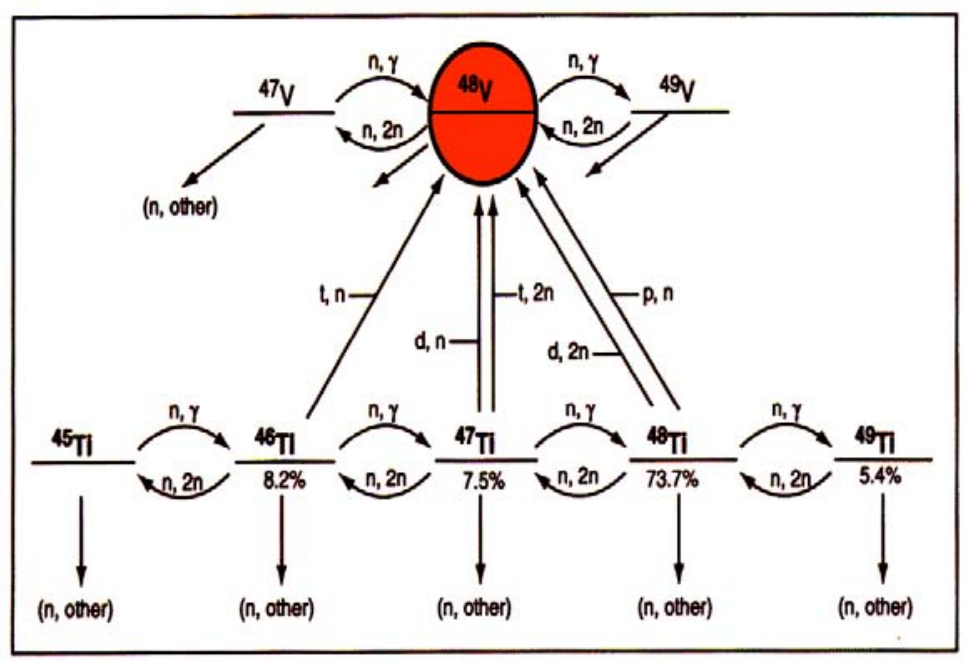

$$
(\mathrm{n}, \text { other })=(n, p)+(n, n p)+(n, \text { alpha })+\ldots
$$


Modeled destruction reactions for ${ }^{48} \mathrm{~V}$ $\left({ }^{48} \mathrm{~V}(\mathrm{n}, \mathrm{p}){ }^{48} \mathrm{Ti}\right.$ dominates at lower energies)

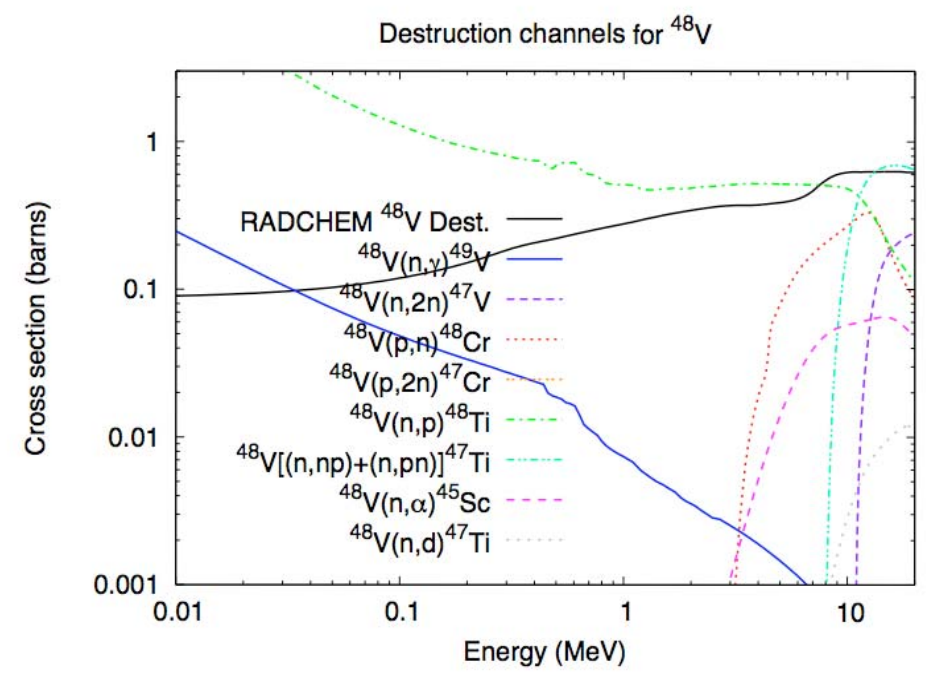

ORELA WORKSHOP - 11

\section{Yttrium (Y) reaction network shows need for} measurements on unstable targets

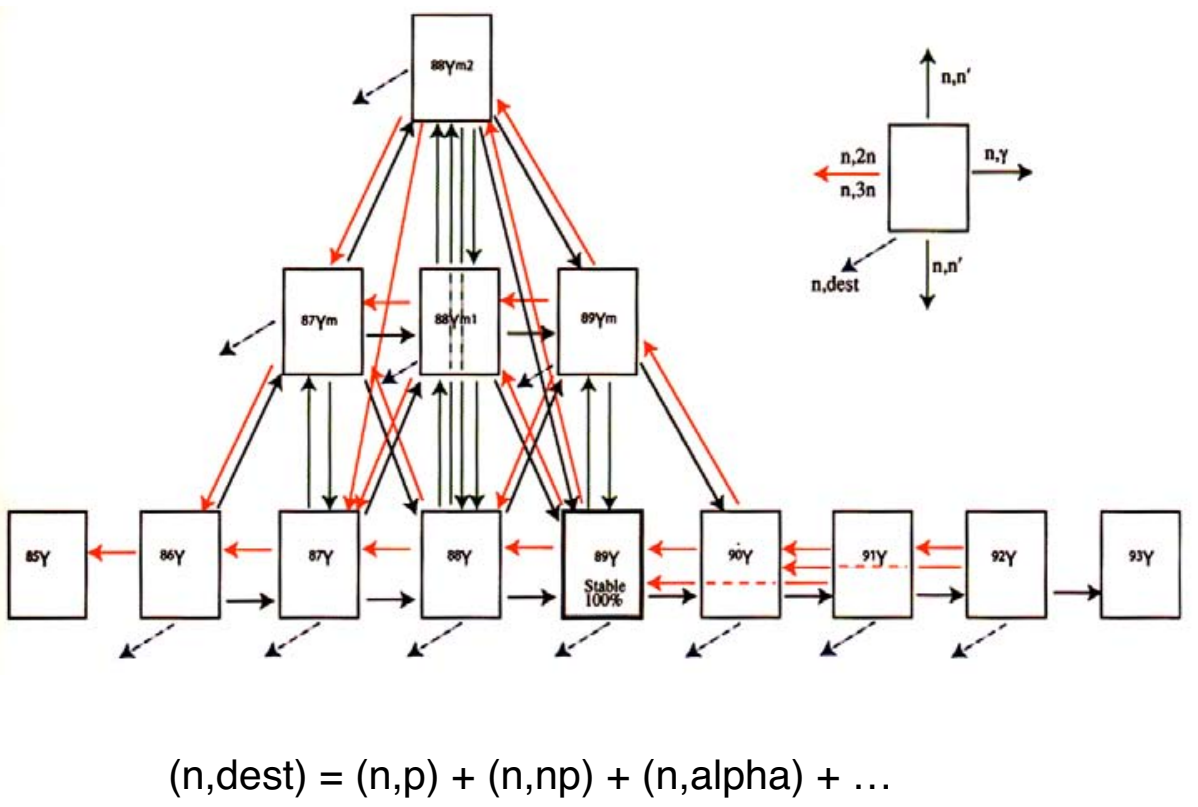

ORELA WORKSHOP - 12 
The nuclear physics goal of the stockpile stewardship program is to bring the cross-section uncertainties to $2-3 \%$ or better for major energy producing reactions and for reactions which are diagnostically useful.

(We recognize that high accuracy for (n,gamma) reactions is a difficult task.)

Needs more refined data and evaluations

${ }^{236} \mathrm{U}(\mathrm{n}$, Fission $)$

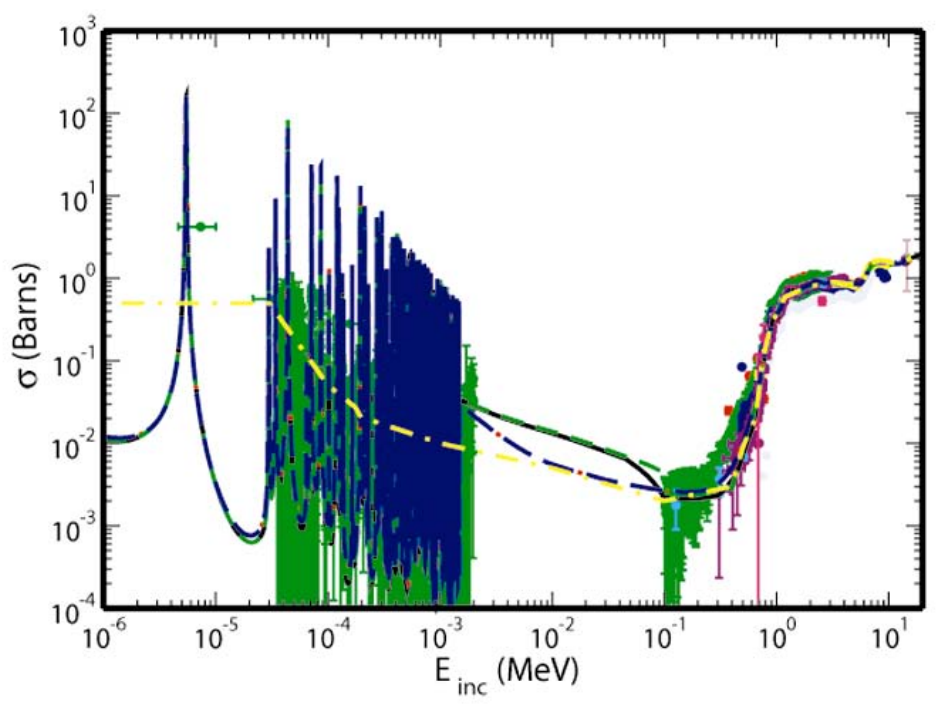


Large scattering in data and evaluations

${ }^{236} \mathrm{U}(\mathrm{n}$, gamma $)$

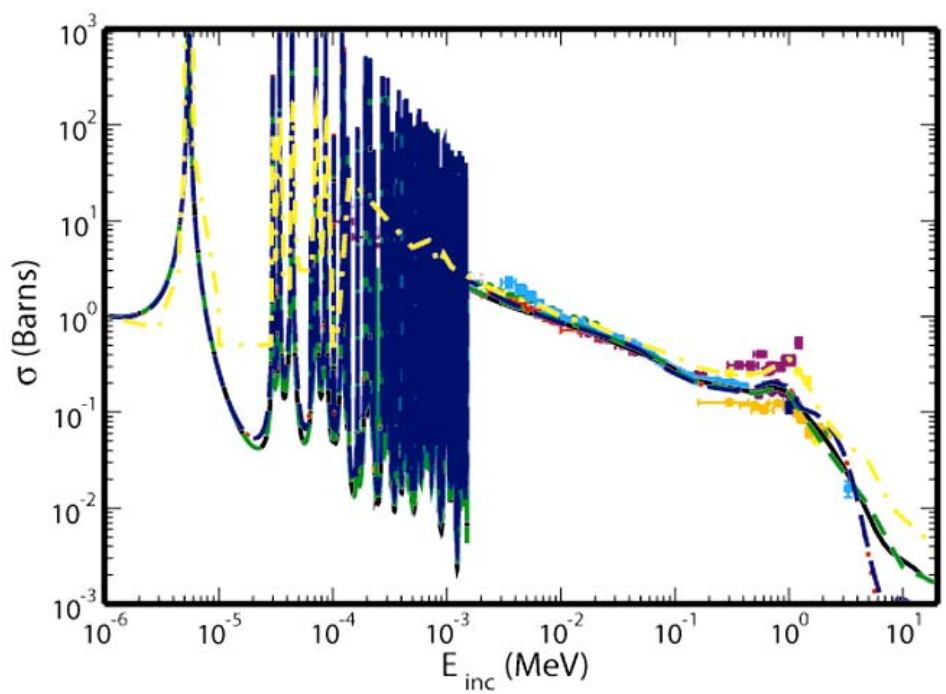

ORELA WORKSHOP - 15

Unacceptable data and evaluations

${ }^{237}$ U(n,Fission)

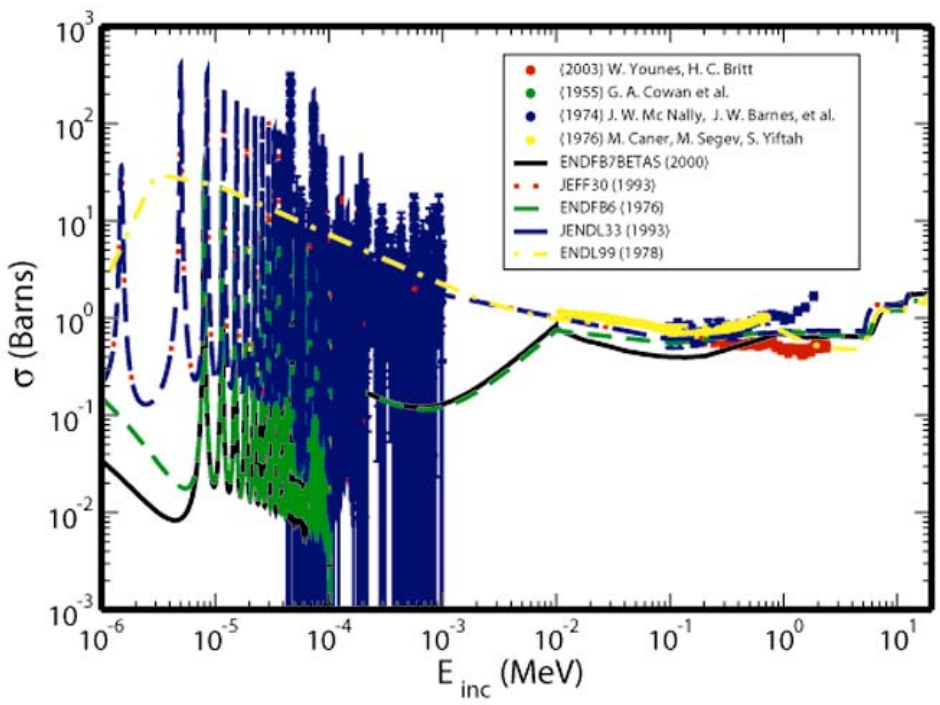


Unacceptable data and evaluations

$$
{ }^{237} \mathrm{U}(\mathrm{n}, \text { gamma })
$$

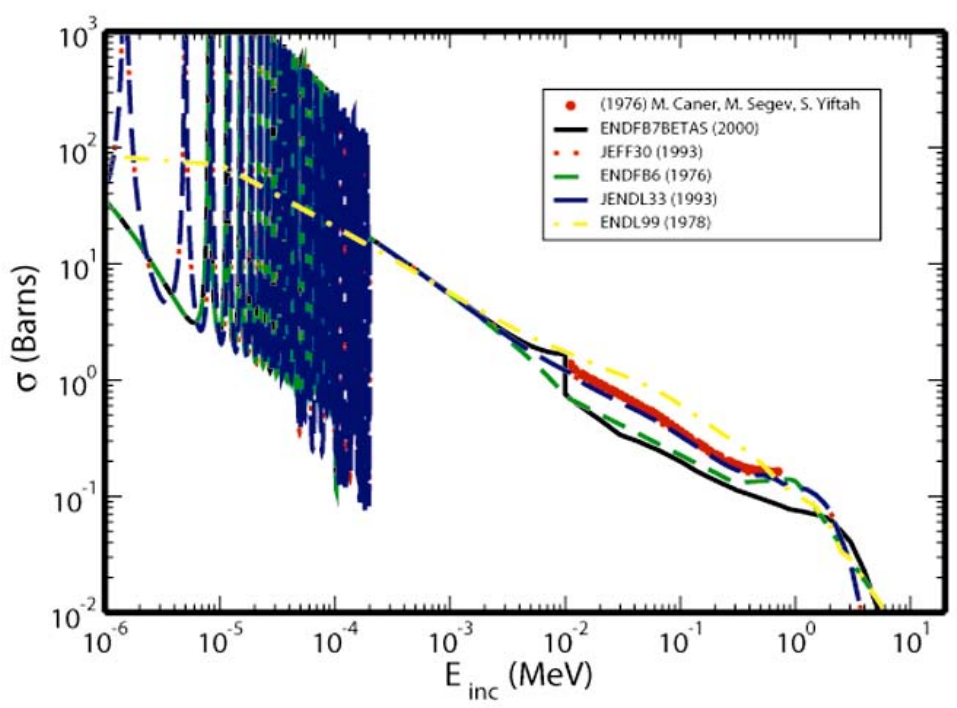

ORELA WORKSHOP - 17

$(n, x)$ cross sections on unstable nuclei can be deduced using decay probability measurements - SURROGATE METHOD (Lee Bernstein et al.)

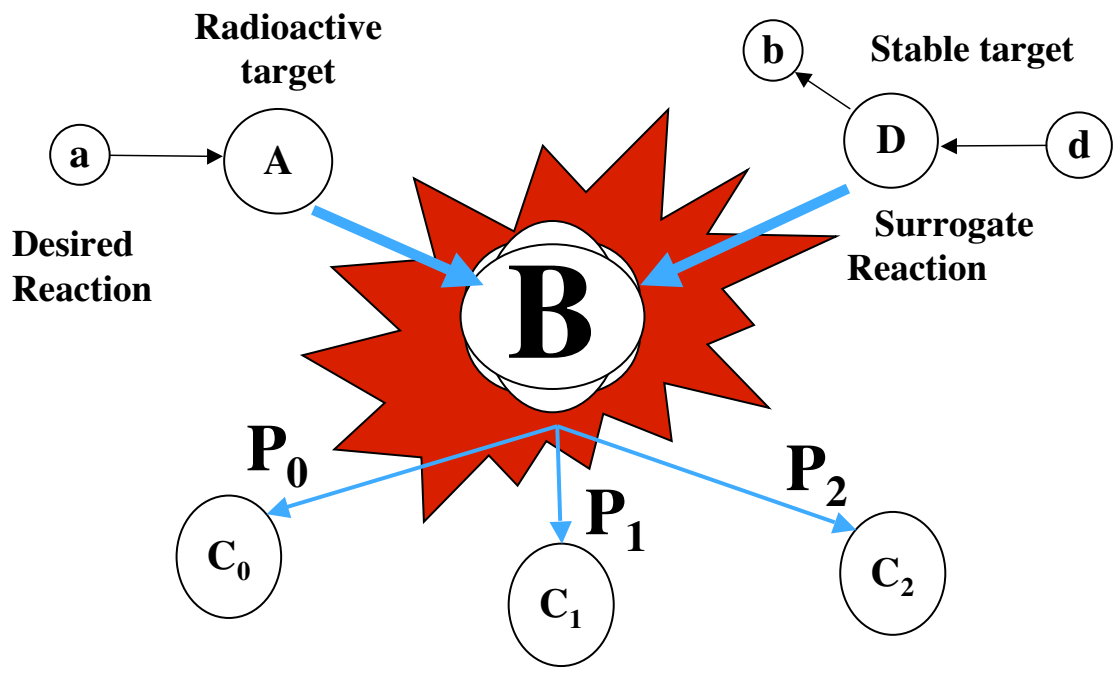


Relative decay probability measurements, when appropriate, provide a more robust result
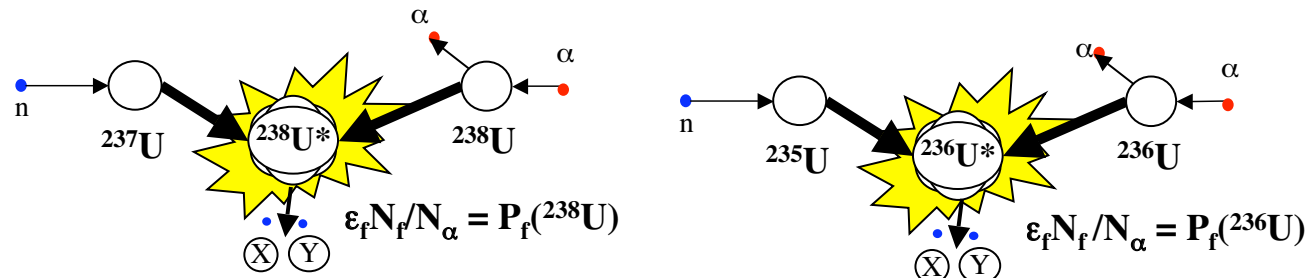

\section{Actinides are ideal for this approach}

ORELA WORKSHOP - 19

${ }^{237} \mathrm{U}(\mathrm{n}, \mathrm{f})$ extracted from the STARS + LIBERACE ${ }^{238} U(\alpha, \alpha f) / 236 U(\alpha, \alpha f)$ ratio

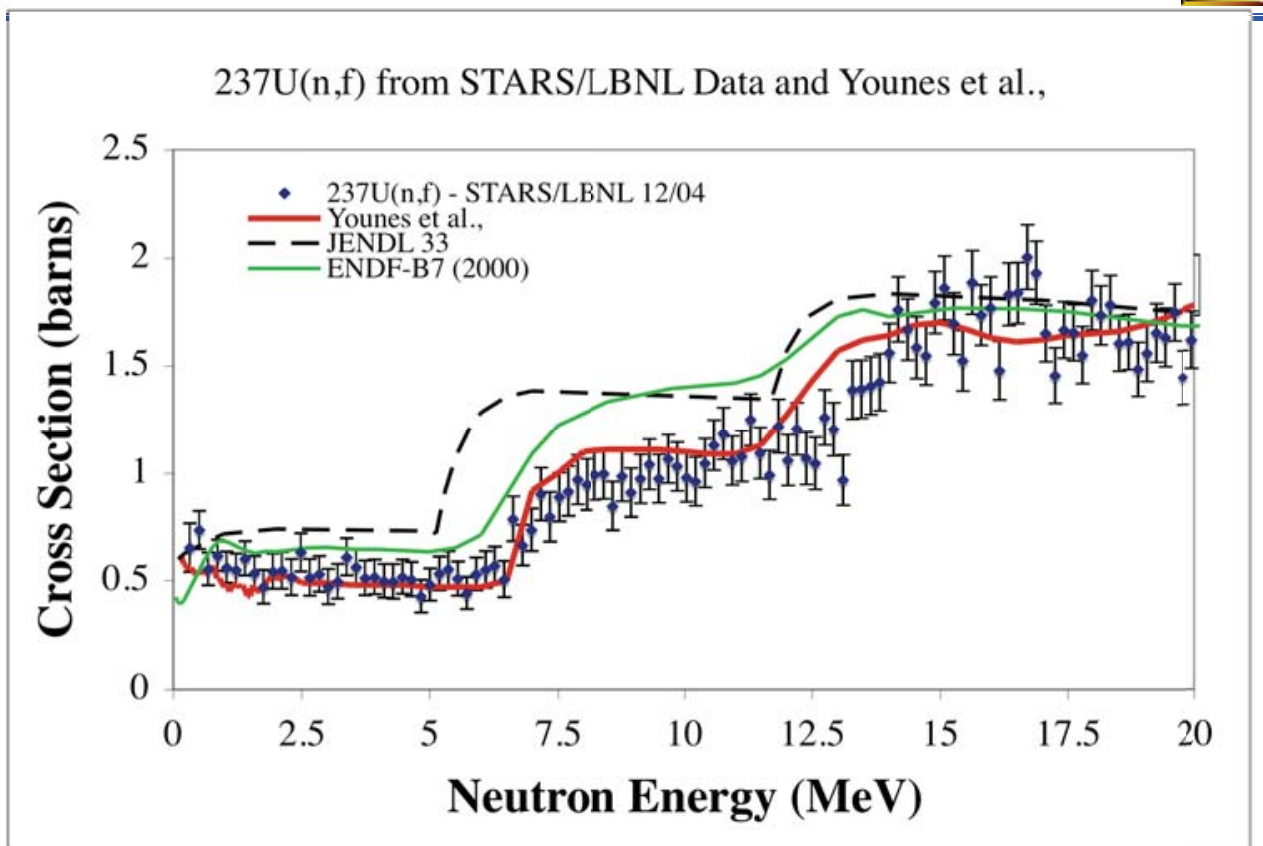

ORELA WORKSHOP - 20 
NA-22 Cross Section Measurement Program

\begin{tabular}{|c|c|c|c|c|}
\hline Reaction & Surrogate & Facility & Status & P.I. \\
\hline $236,238 \mathrm{U}(\mathrm{d}, \mathrm{pf})$ & $236,238 U(n, f)$ & Yale & Published & Plettner (Yale) \\
\hline $236,238 \mathrm{U}\left(\mathrm{d}, \mathrm{d}^{\prime} \mathrm{f}\right)$ & 235,237U(n,f) & Yale & Published & Plettner (Yale) \\
\hline${ }^{236,238} \mathrm{U}\left(\mathrm{d}, \mathrm{d}^{\prime} \mathrm{f}\right)$ & ${ }^{235,237} U(n, f)$ & Yale & Published & Plettner (Yale) \\
\hline${ }^{236,238} \mathrm{U}\left(\alpha, \alpha^{\prime} f\right)$ & ${ }^{235,237} U(n, f)$ & LBNL & Write-up & Burke (LLNL) \\
\hline${ }^{236,238} \mathrm{U}\left(\alpha, \alpha^{\prime} \times n\right)$ & 235,237U(n,xn) & LBNL & Analysis & Burke (LLNL) \\
\hline${ }^{234,238} \mathrm{U}\left({ }^{7} \mathrm{Li},{ }^{5} \mathrm{Li}\right)$ & ${ }^{235,239} U(n, x n)$ & LBNL & Fielded & Ly les (UCB-NE) \\
\hline${ }^{236,238} \mathrm{U}\left({ }^{3} \mathrm{He}, \alpha^{\prime}\right)$ & $234,236 \mathrm{U}(\mathrm{n}, \mathrm{x})$ & LBNL & Proposed & Burke (LLNL) \\
\hline${ }^{239} \mathrm{Pu},{ }^{237} \mathrm{~Np}\left({ }^{6} \mathrm{Li}, \alpha\right)$ & ${ }^{241} \mathrm{Am},{ }^{239} \mathrm{Pu}$ & LBNL & Proposed & New P.D. \\
\hline
\end{tabular}

ORELA WORKSHOP - 21

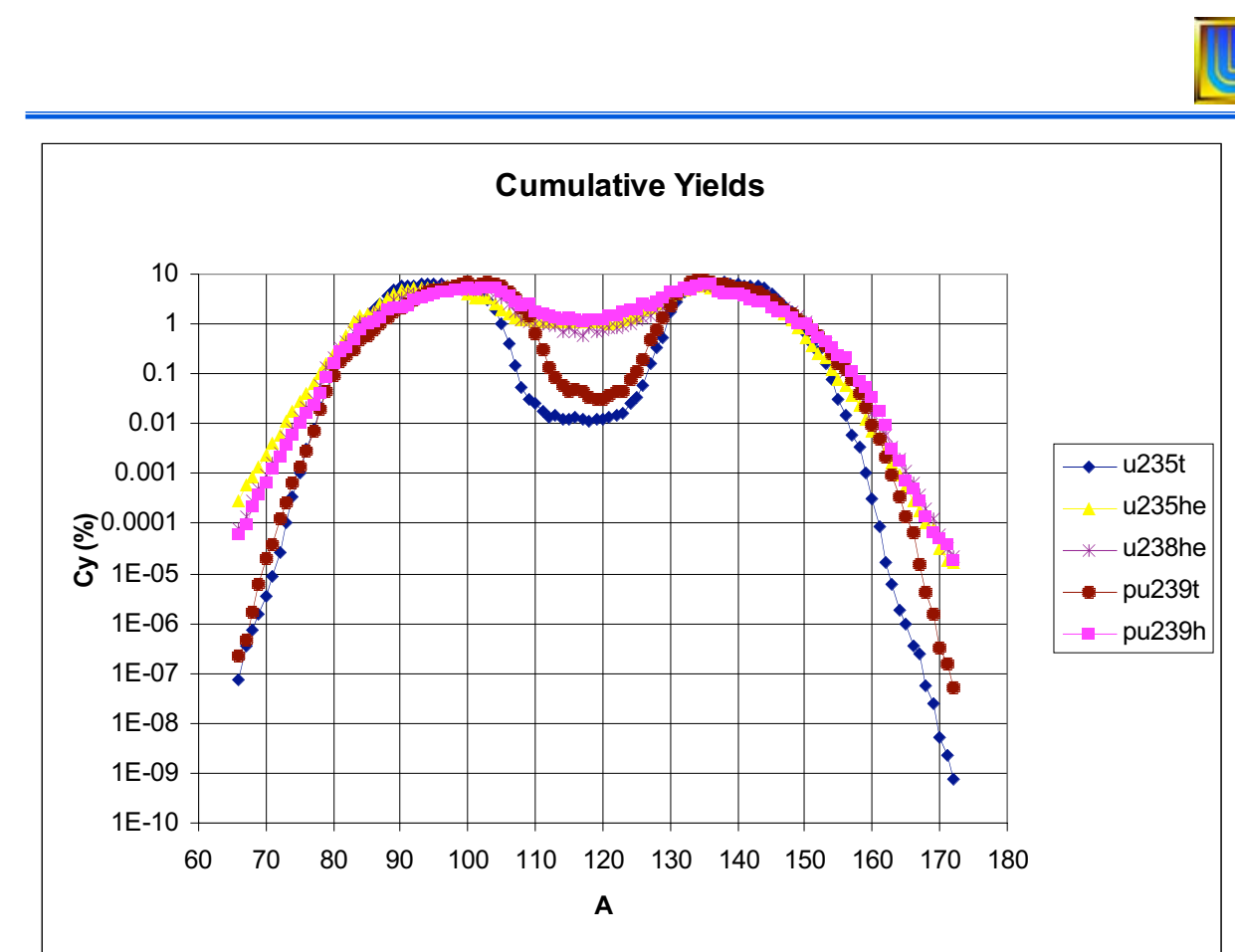

ORELA WORKSHOP - 22 
- More accurate fission and capture cross sections of actinides (uranium, plutonium, americium, neptunium).

- Capture cross sections in the europium and lutetium regions. (151Eu, 153Eu, 151Gd, 175Lu)

- Total cross sections (keV range) around mass 50. (48Ti, 48V)

- Angular distributions(elastic and inelastic) on weapons materials for neutron and charged-particle transport in codes.

- Nuclear data and cross sections on unstable targets and isomeric states (radiochemical detectors). (88Y, $89 \mathrm{Zr}, 170 \mathrm{Tm}, 171 \mathrm{Tm}, 173 \mathrm{Lu}$, 174Lu)

- Measure and model/evaluate $n+$ Li reactions

ORELA WORKSHOP - 23

\section{Final Words}

- Our needs are broad, but uncertainty reduction and quantification are the central task in nuclear data and cross section work. This applies not only to measurements but modeling as well.

- ORELA could certainly contribute to fission, capture and total cross-section measurements and evaluations for both weapons physics and diagnostics. 


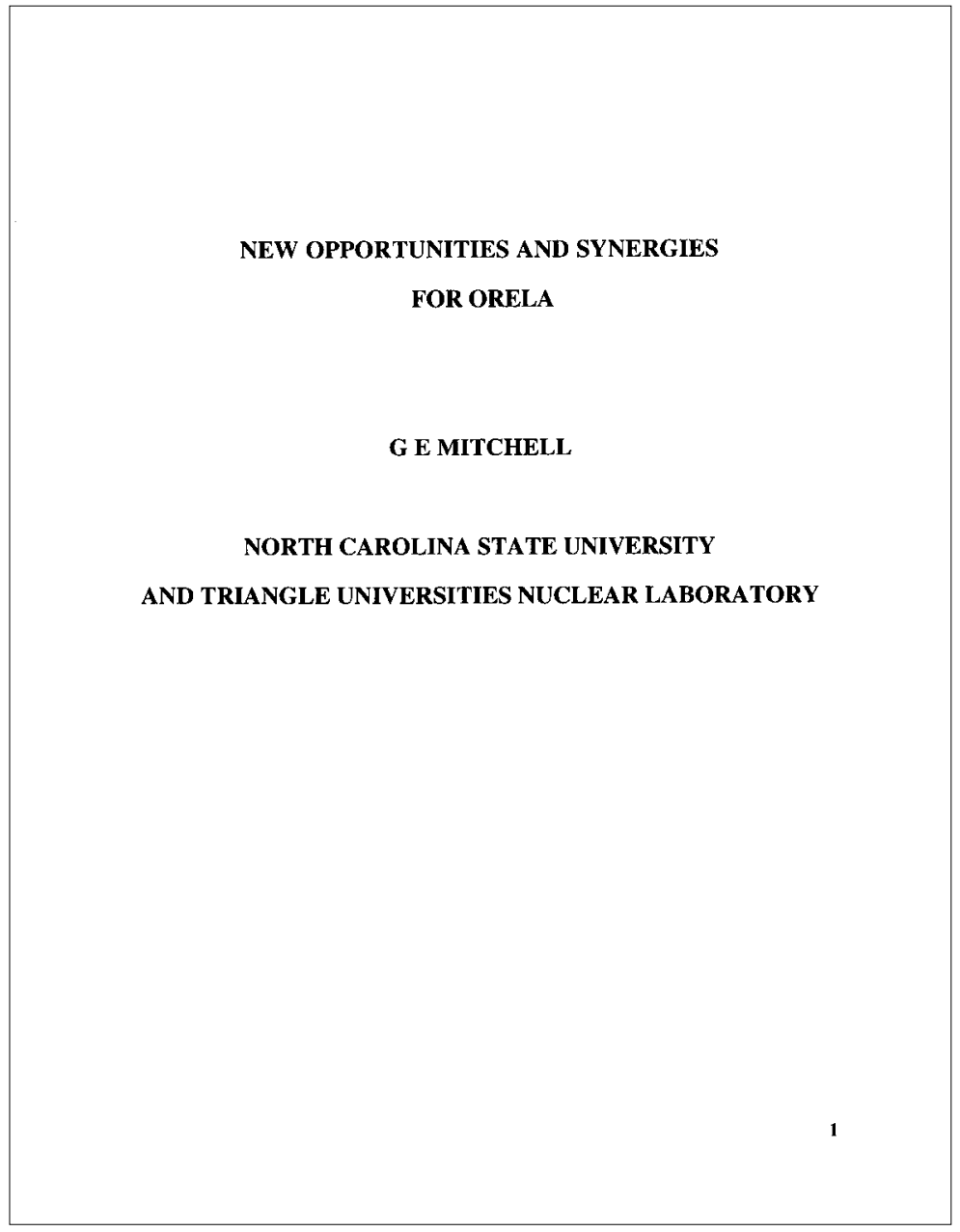

\begin{tabular}{|c|}
\hline \\
OUTLINE \\
HISTORY - PAST AND PRESENT SIGNIFICANCE OF \\
NEUTRON RESONANCES \\
PRESENT NEUTRON RESONANCE DATA \\
A -- MISSING LEVEL CORRECTIONS \\
B - IMPLICATIONS FOR DATA QUALITY \\
COMPLEMENTARY STUDIES \\
A - NEUTRON TOTAL CROSS SECTIONS AT ORELA \\
B - NEUTRON CAPTURE CROSS SECTIONS AT \\
DANCE \\
SUMMARY AND CONCLUSIONS \\
\\
\end{tabular}



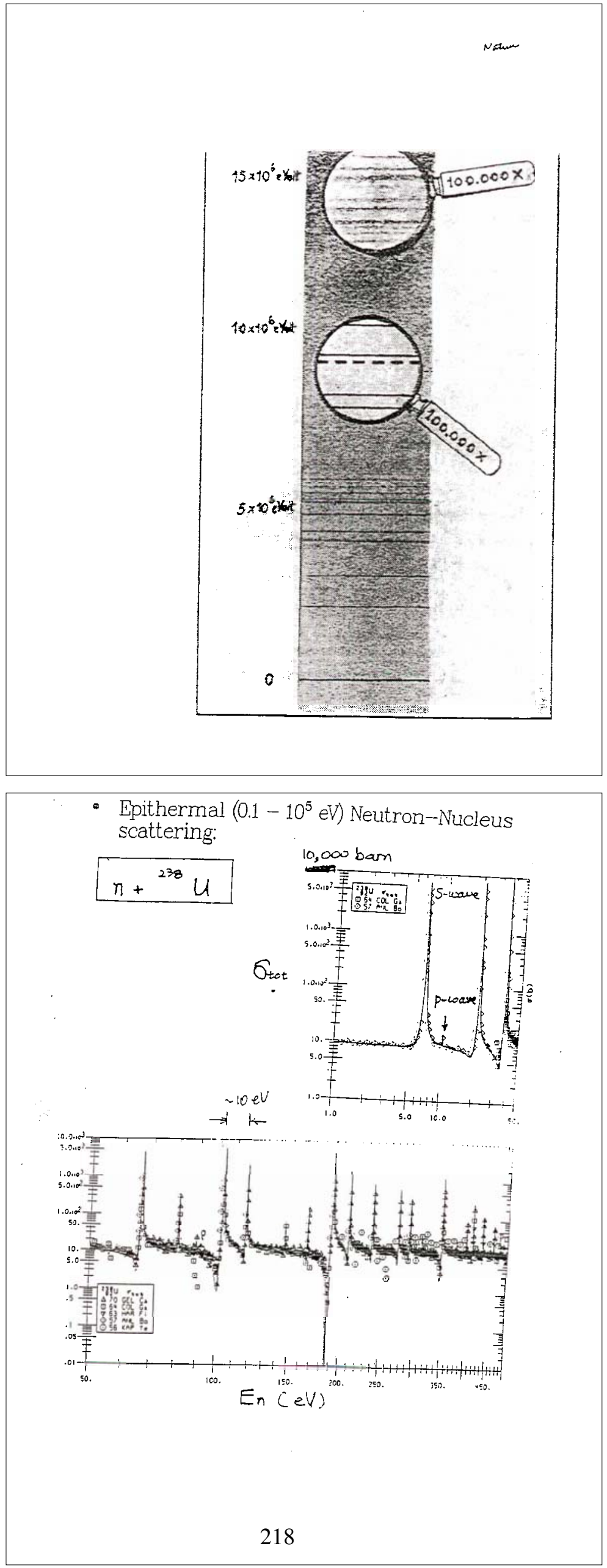

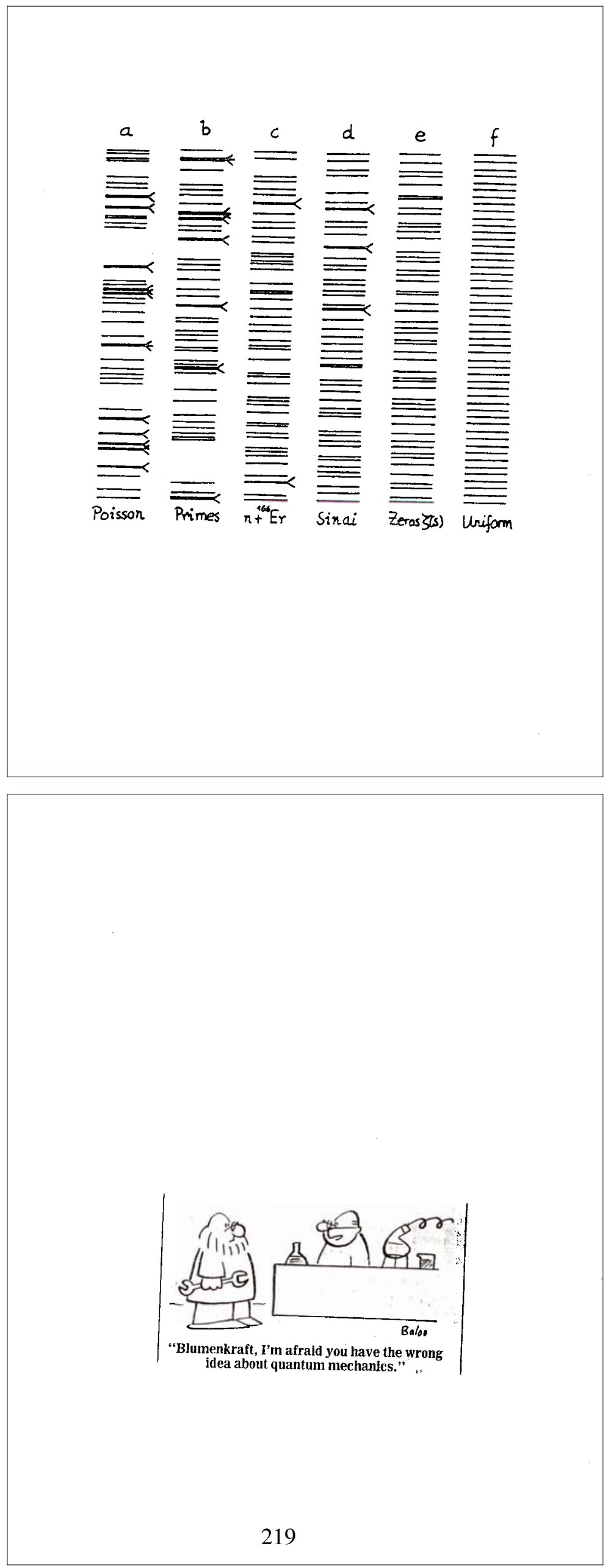
STATISTICAL THEORY OF THE ENERGY

LEVELS OF COMPLEX SYSTEMS. V

M. L. MEHTA AND F. J. DYSON

J. MATH. PHYS. 4 (1963)

PROBLEM H. STATISTICAL EFFECTS

OF MISSING AND SPURIOUS LEVELS

...DESIRABLE TO MAKE THE RESULTS ... MORE PRECISE BY CALCULATING QUANTITATIVELY THE EFFECTS OF MISSING AND SPURIOUS LEVELS. TO CARRY THROUGH SUCH CALCULATIONS WOULD NOT BE DIFFICULT, ONLY RATHER LABORIOUS.

Random Matrix Theory (RMT)

$\Rightarrow$ Assume RMT

1. Reduced widths obey Porter-Thomas distribution

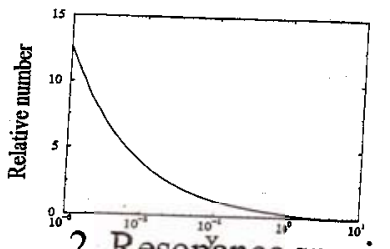

$$
P(y) d y=\frac{1}{\sqrt{2 \pi y}} \exp \left(-\frac{y}{2}\right) d y
$$

$$
y=\frac{\gamma^{2}}{\left\langle\gamma^{2}\right\rangle}
$$

2. Resonance spacings are distributed according to Wigner distribution

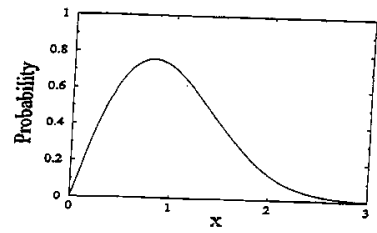

$$
P(x) d x=\frac{\pi x}{2} \exp \left(-\frac{\pi x^{2}}{4}\right) d x
$$$$
x=\frac{D}{\langle D\rangle}
$$ 
Levels with $y<y_{0}=\frac{\gamma_{0}^{2}}{\left\langle\gamma^{2}\right\rangle}$ are undetectable

Standard method:

$\left\{\gamma_{i}^{2}\right\}, i=1, N_{0} \Rightarrow\left\langle\gamma^{2}\right\rangle=\frac{\Sigma \gamma_{i}^{2}}{N_{0}}$

$----\rightarrow y_{\min }=\frac{\gamma_{\min }^{2}}{\left\langle\gamma^{2}\right\rangle}$

$\mathbf{M F}=$ Missing fraction $=\int_{0}^{y_{\min }} P(y) d y$

MS = Missing strength $=\int_{0}^{y_{\min }} y P(y) d y$

$\leftarrow--\left\langle\gamma^{2}\right\rangle=\frac{\sum \gamma^{2}+\mathrm{MS}}{N_{0} /(1-\mathrm{MF})}$

$\langle\Gamma D\rangle=0 \Rightarrow$ use spacing analysis as independent test

Spacing: minimal effect due to nonstatistical phenomena. However, levels missed at random therefore analysis is harder to formulate

Question:

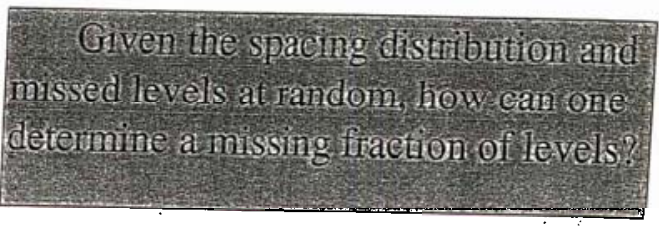


A. Perfect sequence $=$ all levels are observed.

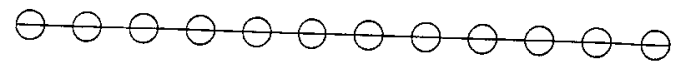

Number of spacings type $0=11$

$(0+1) 11=11$

B. Imperfect sequence $=$ levels are missing.

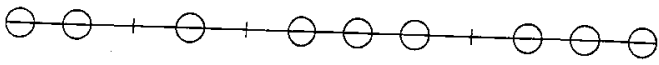

Number of spacings type $0=5$

$(0+1) 5+(1+1) 3=11^{\text {type } 1=3}$

C. Imperfect sequence = levels are missing.

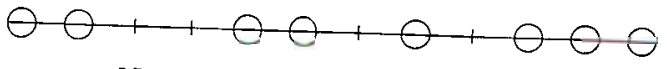

Number of spacings type $0=4$

type $1=2$

type $2=1$

$(0+1) 4+(1+1) 2+(2+1) 1=11$

Expand the spacing distribution in terms of higher order distributions:

$$
\mathrm{P}(z) d z=\sum_{k} a_{k} \lambda \mathrm{p}(k, \lambda z) d z
$$

\section{Normalizations}

$$
\begin{array}{ll}
\int_{0}^{\infty} \mathrm{P}(z) d z=1 & \int_{0}^{\infty} z \mathrm{P}(z) d z=1 \\
\int_{0}^{\infty} \mathrm{p}(k, z) d z=1 & \int_{0}^{\infty} z \mathrm{p}(k, z) d z=k+1
\end{array}
$$

lead to conditions:
(a). $\sum_{k=0}^{\infty} a_{k}=1$
(b). $\sum_{k=0}^{\infty} a_{k}(1+k)=\lambda$ 

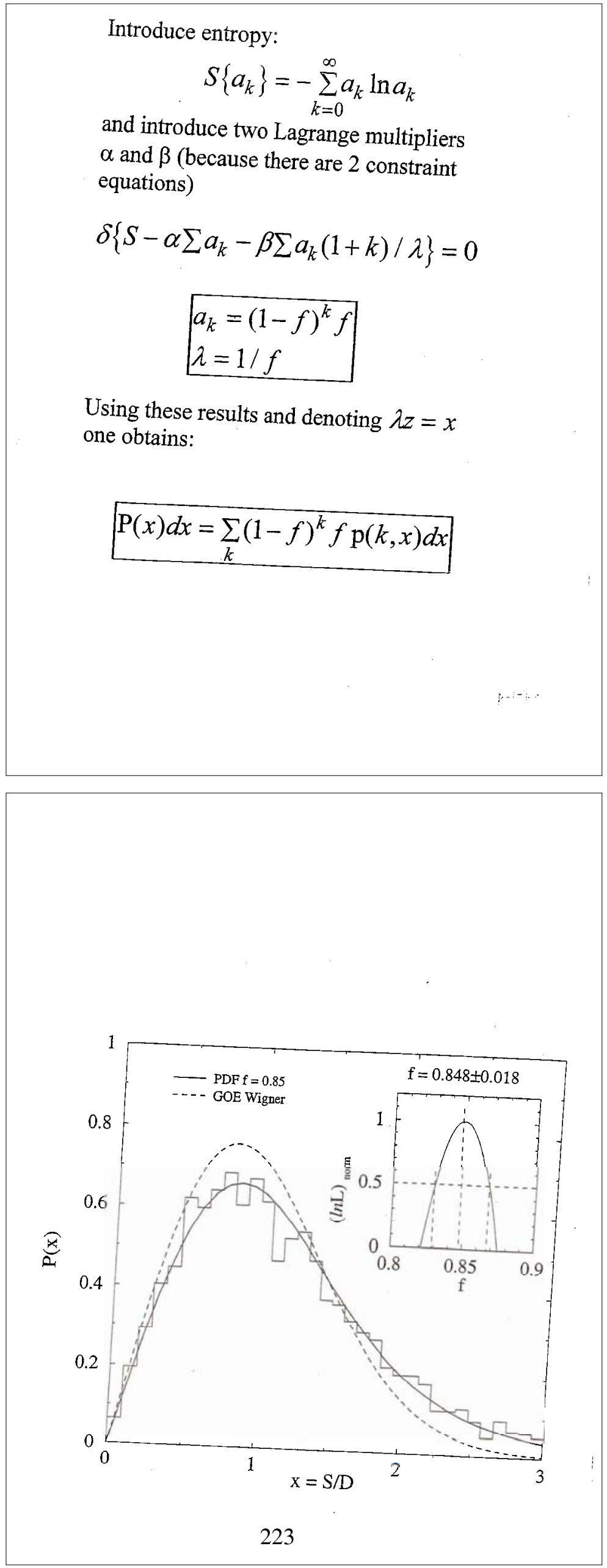

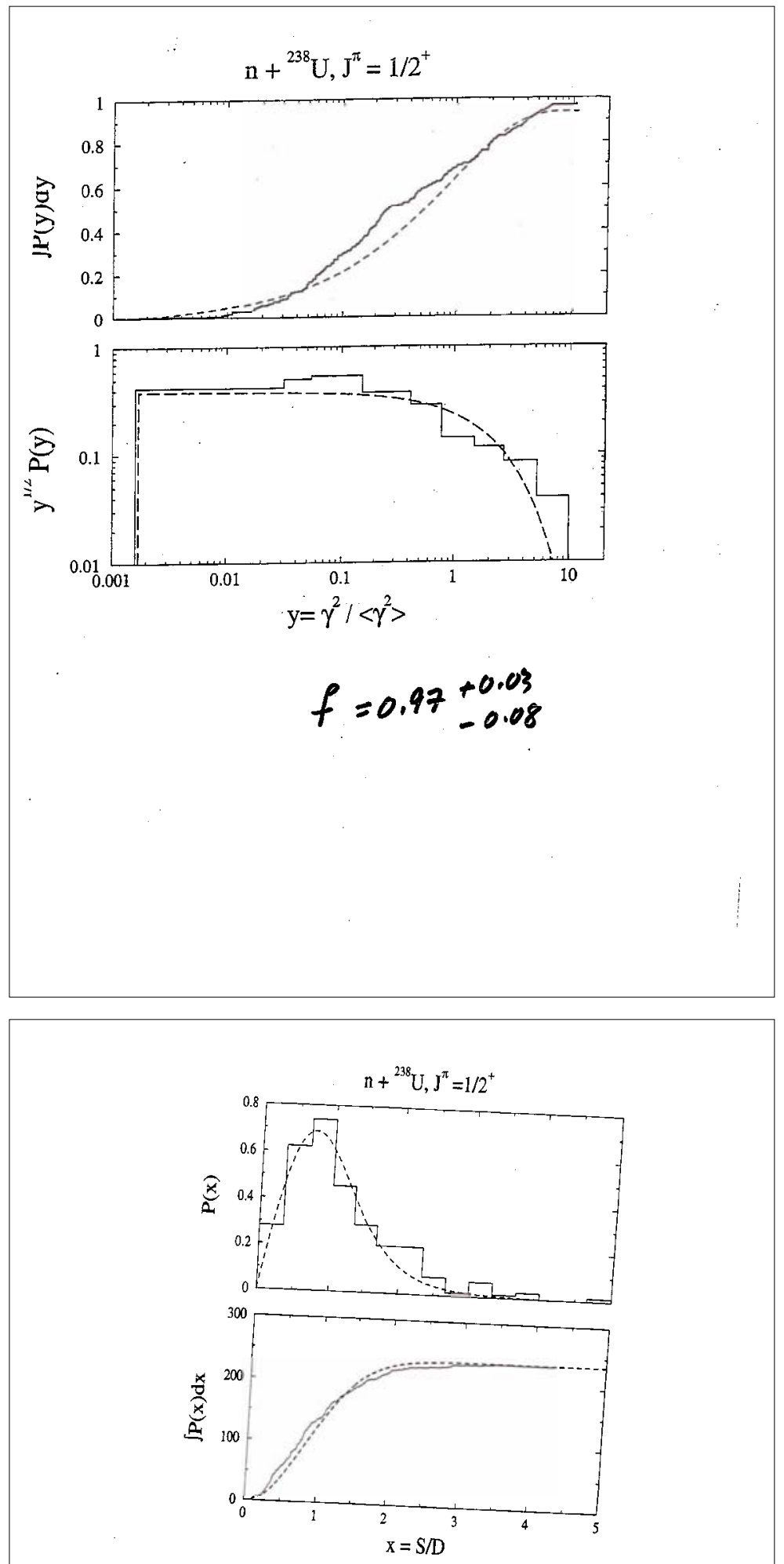

Method 2: Spacing analysis $f=0.89 \pm 0.06$ less than $f=0.97_{-0.08}^{+0.03}$ from method 1 . 
EVALUATION OF PRESENT DATA

f IS FRACTION OF TRUE LEVELS OBSERVED

S-WAVE RESONANCES

LARGE SAMPLE SIZES $(N>100)$

I FOR TWO METHODS DIFFERS BY 5\%

\section{S-WAVE RESONANCES}

SMALL SAMPLE SIZES $(\mathbf{N}=\mathbf{3 0 - 6 0})$

I FOR TWO METHODS DIFFERS BY 8\%

P-WAVE RESONANCES

SMALL SAMPLE SIZES $(\mathbf{N}<\mathbf{5 0})$

f FOR TWO METHODS DIFFERS BY 14\%

(NOTE THAT THE DIFFERENCES IN THE CORRECTION (1 - f) IS MUCH LARGER:

$20 \%-60 \%$

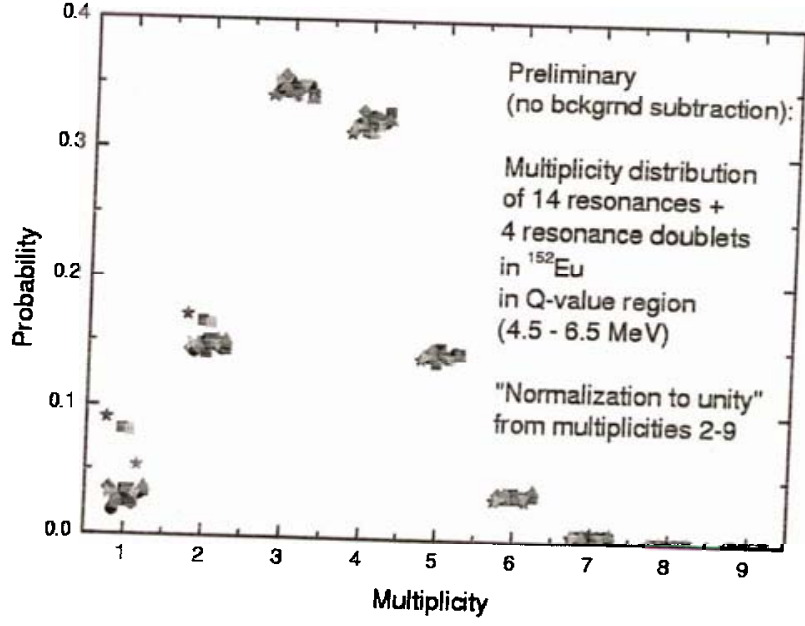




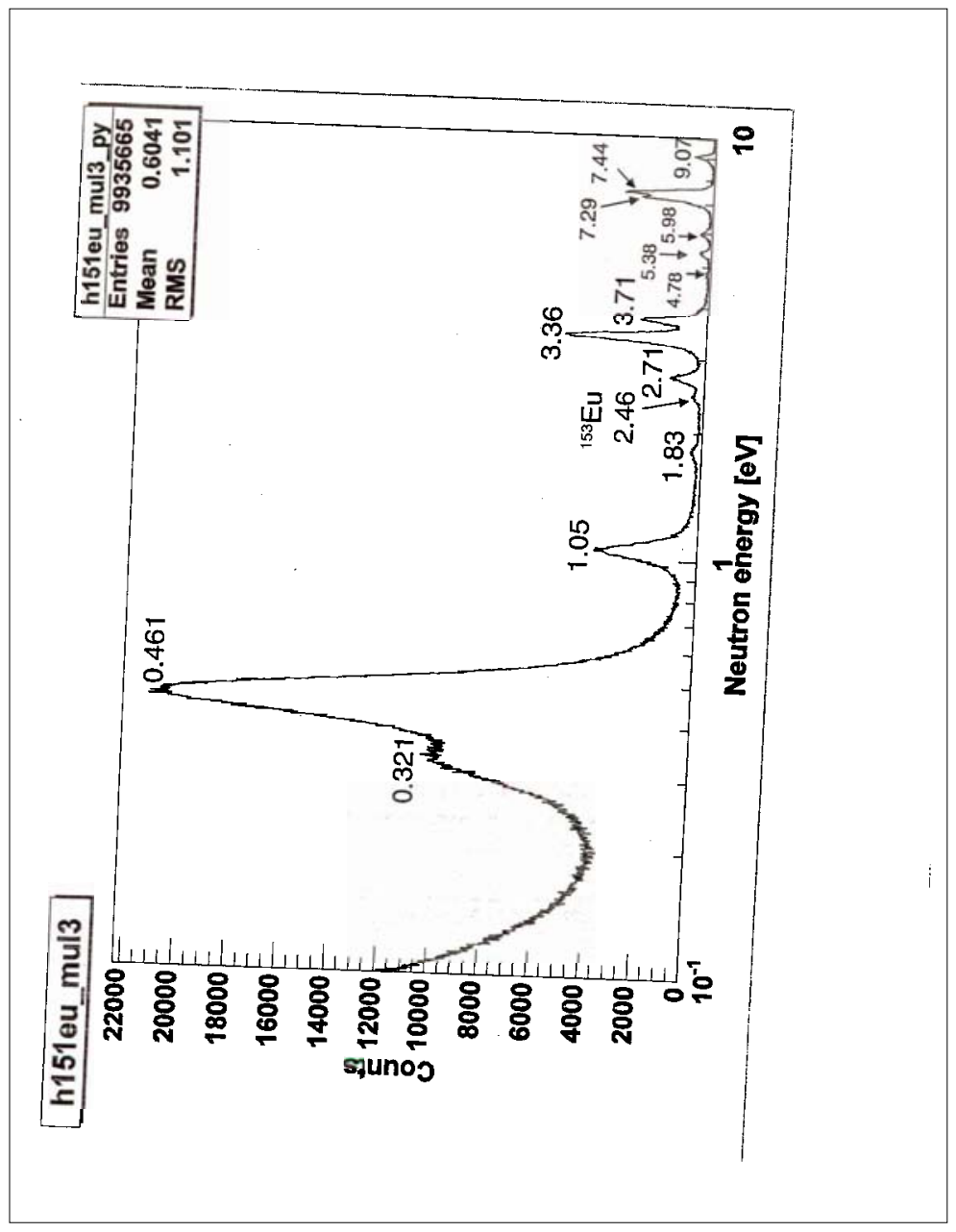

\begin{tabular}{|c|}
\hline SYNERGY BETWEEN ORELA AND DANCE \\
\hline $\begin{array}{c}\text { (DETECTOR ARRAY FOR NEUTRON CAPTURE } \\
\text { EXPERIMENTS) }\end{array}$ \\
\hline $\begin{array}{c}\text { LARGE ARRAY OF BARIUM FLUORIDE CRYSTALS } \\
162 \text { SEGMENTS AND } 160 \text { DETECTORS }\end{array}$ \\
\hline $\begin{array}{c}\text { VERY HIGH EFFICIENCY } \\
\text { THEREFORE CAN USE VERY SMALL SAMPLES } \\
\text { INCLUDING RADIOACTIVE TARGETS }\end{array}$ \\
\hline $\begin{array}{c}\text { THESE CAPTURE DATA COMBINED WITH } \\
\text { NEUTRON TOTAL CROSS SECTION } \\
\text { MEASUREMENTS PRODUCE BEST POSSIBLE DATA } \\
\text { SET }\end{array}$ \\
\hline $\begin{array}{l}\text { SPECIFIC CASE EUROPIUM } 151 \text { AND } 153 \\
\text { HAVE CAPTURE DATA - } \\
\text { WILL MEASURE TOTAL CROSS SECTION } \\
\text { AT ORELA }\end{array}$ \\
\hline 226 \\
\hline
\end{tabular}




\section{UT-BATTELLE \\ Future of Nuclear Energy in the United States \\ Jim Rushton and Jeff Binder \\ Presentation to ORELA Workshop \\ July 14, 2005

\section{Future of Nuclear Energy}

Drivers for Building New Nuclear Plants

- Need for nuclear power plants

- Meet electricity demand

- Limit GHG emissions

- Nuclear is cost competitive

- Industry performance

- Life extension/License renewals

- Industry restructuring

- Gas price increase

- New plant construction/planning

- DOE/industry path forward 


\section{Why Nuclear Energy?...}

We Depend on it Today
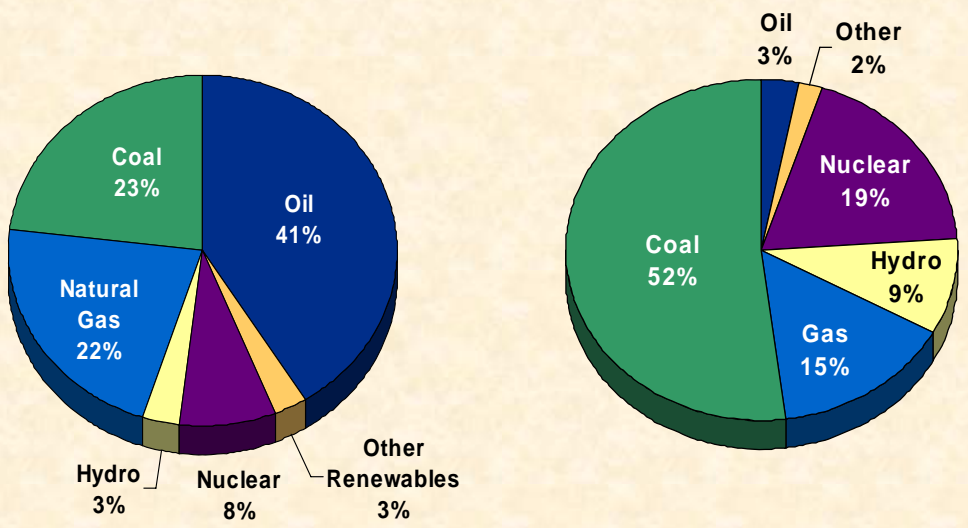

Energy

Production

U.S. Electricity Production

Source: EIA

OAK RIDGE NATIONAL LABORATORY

U. S. DEPARTMENT OF ENERGY

\section{Economic, Energy, and Electricity Growth (1973-2025)}

DOE/Industry goal for 2020 to maintain $\sim 30 \%$ clean generation

$\bullet 10,000 \mathrm{MWe}$ upgrade to existing plants

-50,000 MWe new nuclear plants

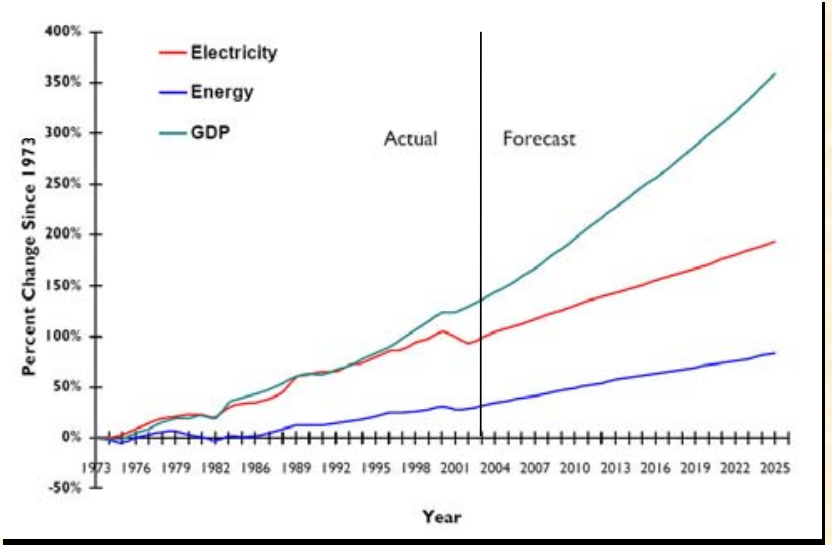




\section{U.S. Generation Capacity Additions}

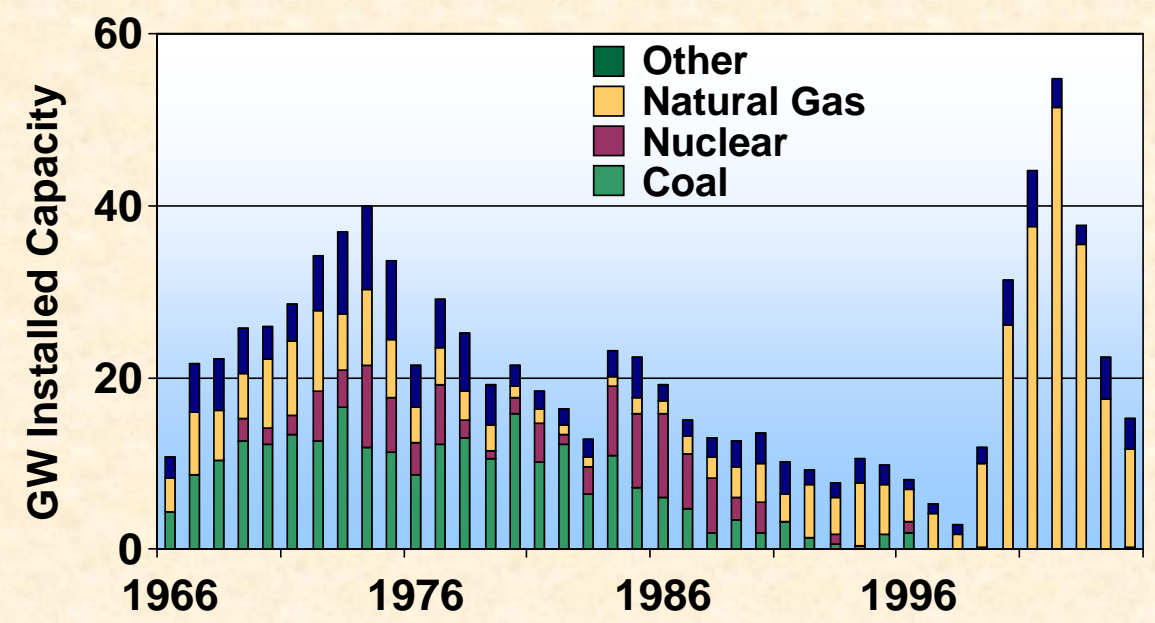

OAK RIDGE NATIONAL LABORATORY

Greenhouse Gas Emissions from Electricity Production Chains ( $\mathrm{gCO}_{2}$ equiv./kWh)

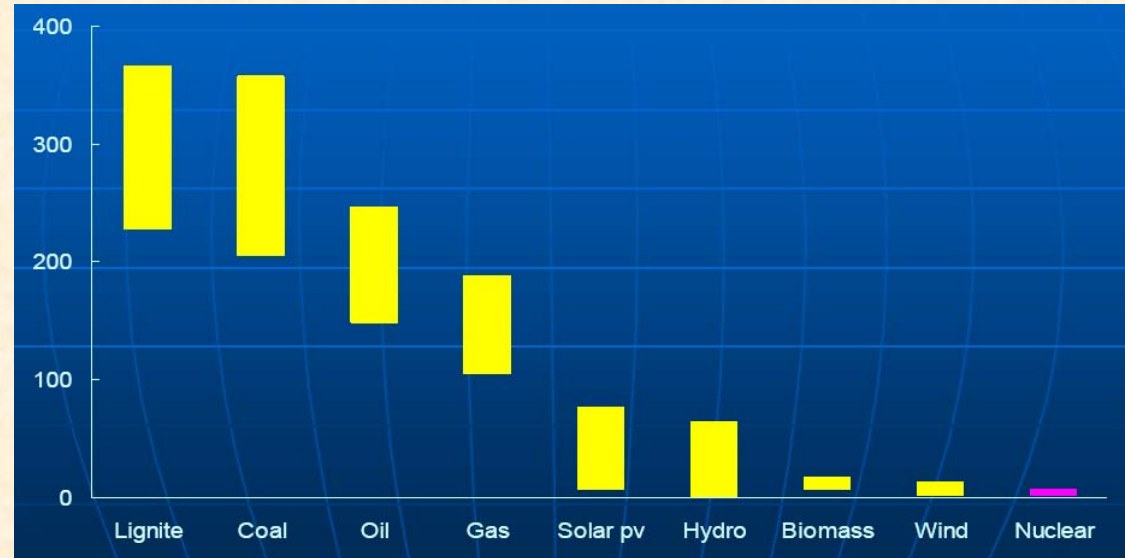




\section{Perspective on USA Nuclear Power and Carbon Emissions (2002)}

Electric sector carbon emissions would be $29 \%$ larger without nuclear power

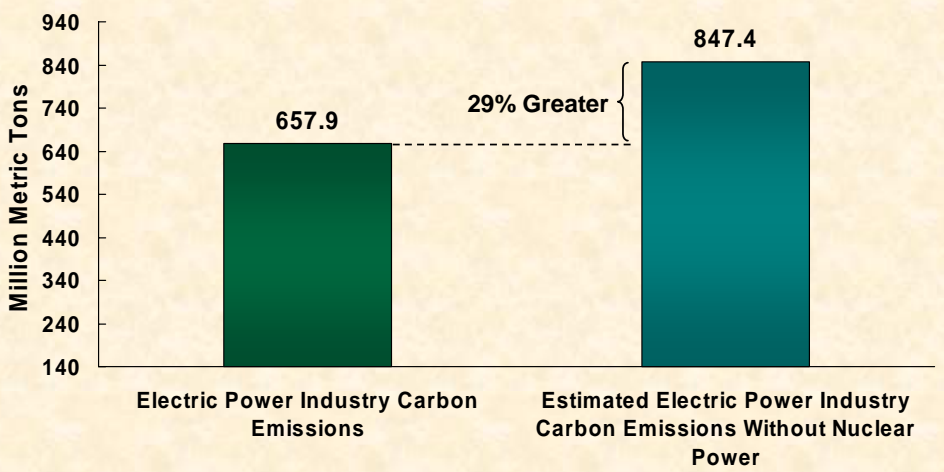

Emissions avoided by nuclear power are calculated using regional fossil fuel emissions rates from EPA CEMS data and individual plant generation data from EIA. Total Emissions are calculated from EPA CEMS data. Last updated 9/15/03.

OAK RIDGE NATIONAL LABORATORY

U. S. DEPARTMENT OF ENERGY

\section{Existing Nuclear Power is Clearly Cost Competitive}

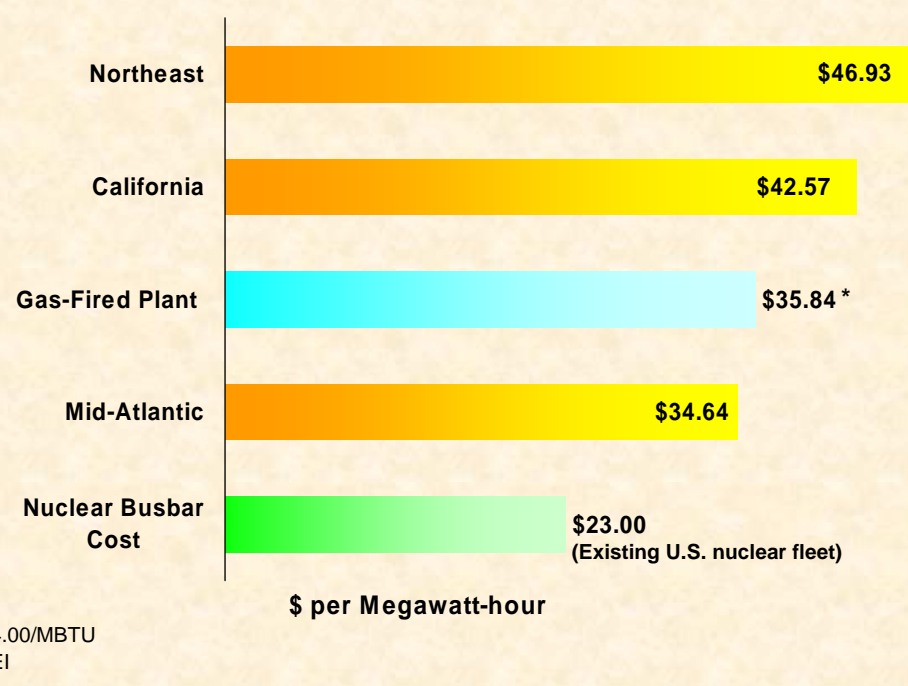

*Gas @ \$4.001

OAK RIDGE NATIONAL LABORATORY

U. S. DEPARTMENT OF ENERGY 


\section{Nuclear Capacity Factors Have Improved Dramatically}

(Equivalent to 23-1000 MWe Plants)

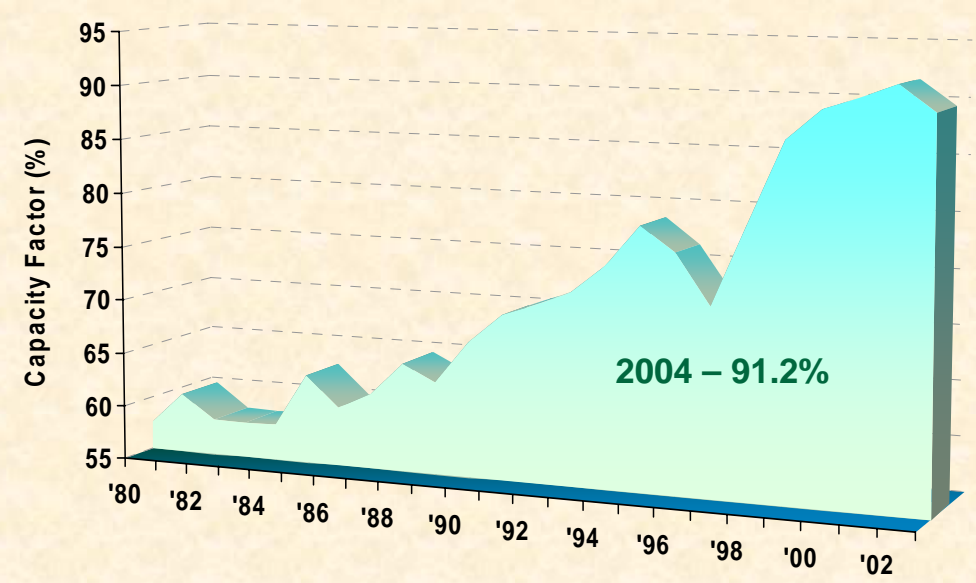

Source: NEI

OAK RIDGE NATIONAL LABORATORY

U. S. DEPARTMENT OF ENERGY

\section{Rising Natural Gas Prices Emphasize} Price Stability of Nuclear Electricity

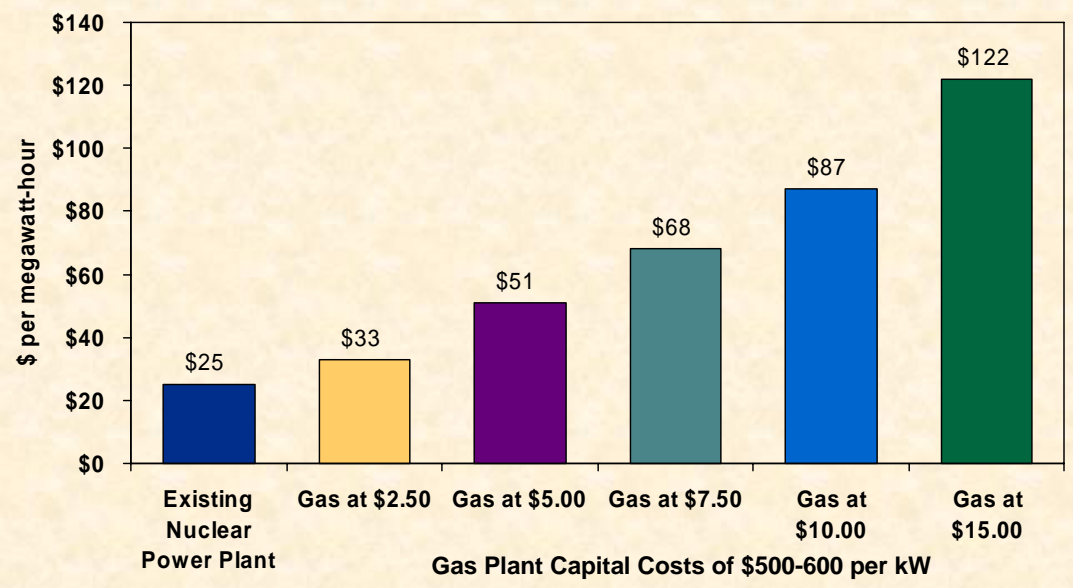

Source: NEI

OAK RIDGE NATIONAL LABORATORY

U. S. DEPARTMENT OF ENERGY 


\section{Most Operating U.S. Nuclear Plants Will Renew Their Licenses - Adds 20 years}

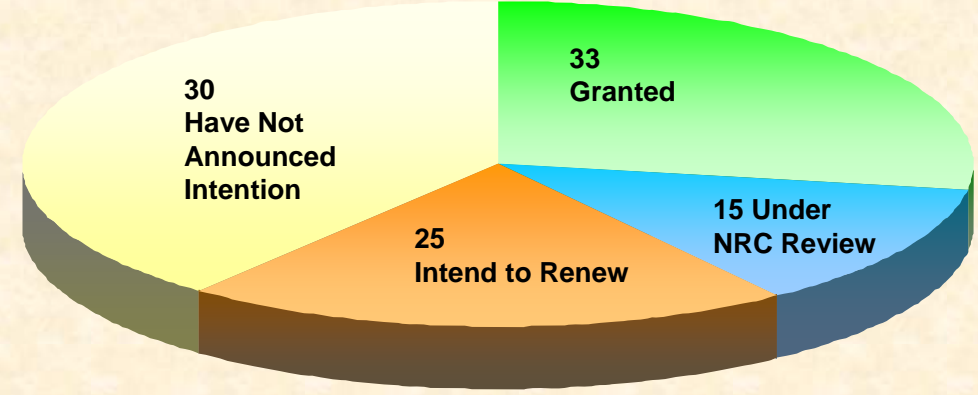

License Renewal Status

Source: NE

OAK RIDGE NATIONAL LABORATORY

U. S. DEPARTMENT OF ENERGY

\section{Restructuring of U.S. Nuclear Industry}

\section{Consolidation of Owners/Operators}

- 12 owners operate $74 \%$ of U.S. nuclear plants

- 4 utilities formed a nuclear management company to operate 8 plants at 6 sites

\section{Vendors}

- 3 nuclear plant vendors [Westinghouse (BNFL), Framatome, GE]

- 3 nuclear fuel vendors [Westinghouse (BNFL), Framatome, Global Fuels (GE)]

- Canadian vendor (AECL) has advanced CANDU (ACR-700) 


\section{International Perspective:}

Competitiveness of Alternatives in France (8\% Discount Rate - €2001)

\begin{tabular}{|c|c|c|c|}
\hline Investment & 口O\&M & Fuel & $\begin{array}{c}\text { R\&D } \\
31.3 \in / M W h\end{array}$ \\
\hline 26.4 €/MWh & \multirow{3}{*}{\multicolumn{2}{|c|}{21.4}} & \\
\hline 4.4 & & & 11 \\
\hline 5.1 & & & 6.3 \\
\hline 16.3 & $\begin{array}{l}4.2 \\
6.1\end{array}$ & & 14 \\
\hline Vuclear & Gas & & Boal \\
\hline
\end{tabular}

Source: French Ministry of Industry 2003

OAK RIDGE NATIONAL LABORATORY

International Perspective:

Competitiveness of Alternatives in Finland (5\% Discount Rate - €2001)

\begin{tabular}{|c|c|c|c|c|c|c|}
\hline €/MWh & Nuclear & Coal & Gas & Peat & Wood & Wind \\
\hline Investment & 13.8 & 7.6 & 5.3 & 10.2 & 13.0 & 40.1 \\
\hline O\&M & 7.3 & 7.4 & 1.5 & 6.5 & 8.2 & 10.0 \\
\hline Fuel & 3.0 & 17.1 & 23.7 & 15.8 & 18.4 & - \\
\hline TOTAL & 24.1 & 32.1 & 30.5 & 32.5 & 39.6 & 50.1 \\
\hline
\end{tabular}

Source: Lappeenrenta University 2001

OAK RIDGE NATIONAL LABORATORY

U. S. DEPARTMENT OF ENERGY 


\section{U.S. Generation Cost Comparison}

University of Chicago Study - August 2004

"The Economic Future of Nuclear Power"

\begin{tabular}{|lcc|}
\hline & \multicolumn{2}{c|}{ LCOE* $^{*}$ \$/MWh (2003) } \\
\cline { 2 - 3 } & No GHG Control & GHG Control \\
\hline Coal & $33-41$ & $83-91$ \\
\hline Gas & $35-45$ & $58-68$ \\
\hline Nuclear & $41-47$ & $41-47$ \\
\hline $\begin{array}{l}\text { Nuclear } \\
\text { (with Incentives) }\end{array}$ & $26-35$ & $26-35$ \\
\hline
\end{tabular}

*LCOE - Levelized Cost of Electricity

**Financing Incentives

- 20\% Investment Tax Credit

- 8 year Production Tax Credit, \$18/MWh

Assumptions $\quad \underline{\text { Coal }}$ Gas $\underline{\text { Nuclear }}$

Capital (\$/MWe) $1200 \quad 590 \quad 1200$ (NOAK)

Fuel (\$/MWh) $\quad 11.26 \quad 23.60^{\star *} \quad 4.35$

$\begin{array}{llll}\text { O\&M (\$/MWh) } & 7.73 & 2.60 & 13.50\end{array}$

Construction (years) $44 \quad 3 \quad 5$

Efficiency (\%) $\quad 30-35 \quad 55-60 \quad-30$

U. S. DEPARTMENT OF ENERGY

UT-BATTELLE

\section{U.S. DOE Nuclear Power 2010 and Generation IV Programs are Addressing Near-Term Regulatory and Long-Term Viability Issues}

\section{NP-2010 Program}

- Eliminate regulatory uncertainties/demonstrate 10CFR52 process (early site permitting and a combined operating license)

- Complete design and engineering of Generation III+ Reactor

Generation IV Nuclear Energy Systems

Program

- Generation IV International Forum

- Concept screening and technology roadmap

- Broad spectrum of advanced system concepts

OAK RIDGE NATIONAL LABORATORY

U. S. DEPARTMENT OF ENERGY

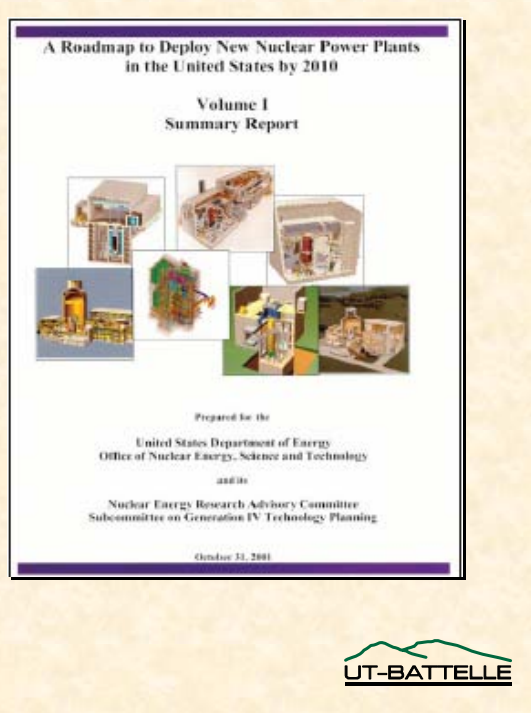




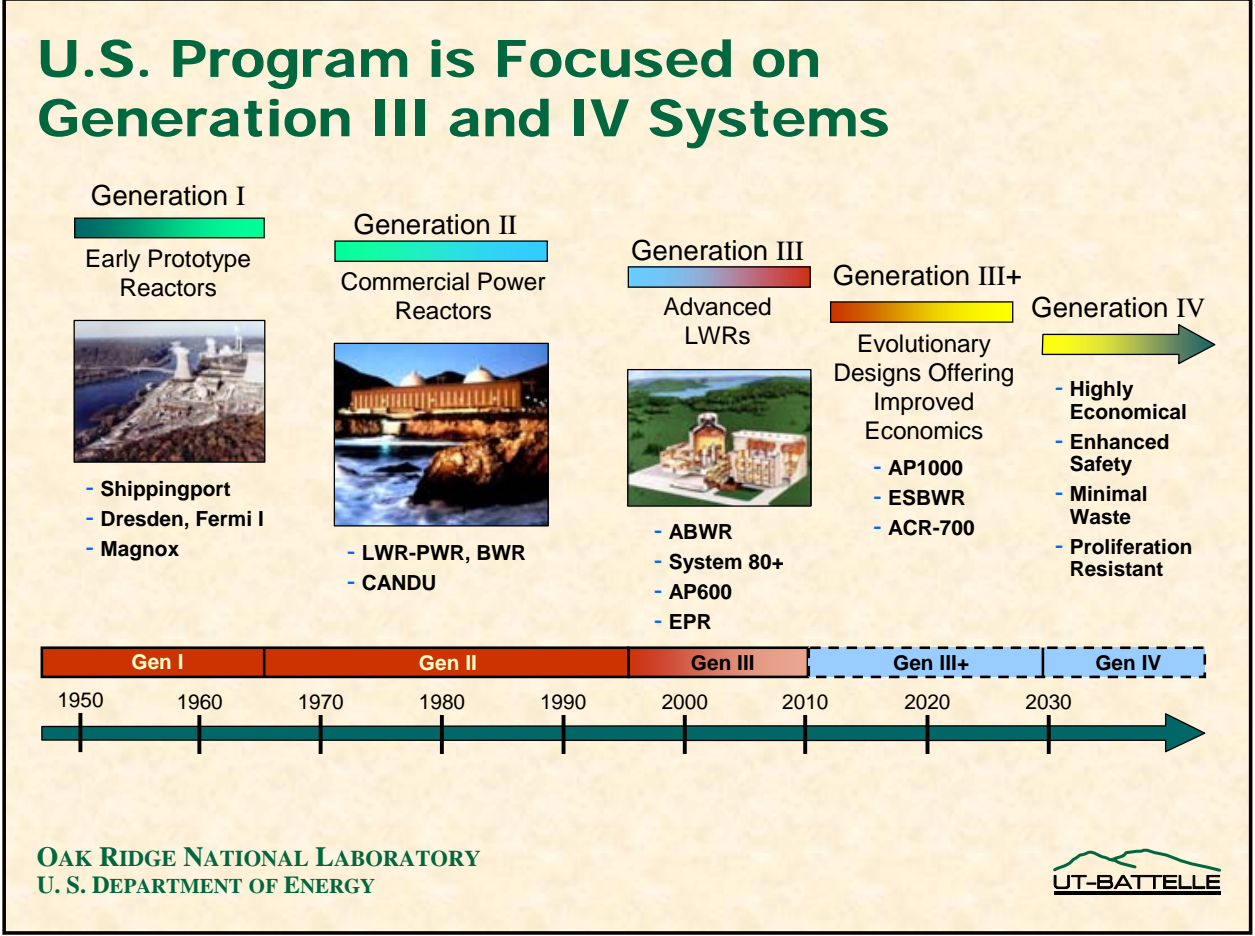

\section{Improved Designs Validating New Licensing Process}

- New process created in 1992 Energy Policy Act

- Regulatory approvals up front, before major investment

- Design certification

- Three reactors (AP600, ABWR, System 80+) already certified

- AP1000 completed U.S. Nuclear Regulatory Commission review; ESBWR, EPR, AECL-700 in pre-review

- Early site approval

- Dominion, Exelon, Entergy have filed applications with the U.S. NRC for early site permits

- Combined construction/operating license

- DOE selected two industry teams to demonstrate process

- TVA evaluating process for advanced reactor/Bellefonte site

- First combined license application expected in 2007 (NuStart Energy Development, LLC)

- First new plant in service approximately 2014 - 2015

OAK RIDGE NATIONAL LABORATORY 


\section{AP1000 Design - Passive Safety and Reduced Numbers of Components}

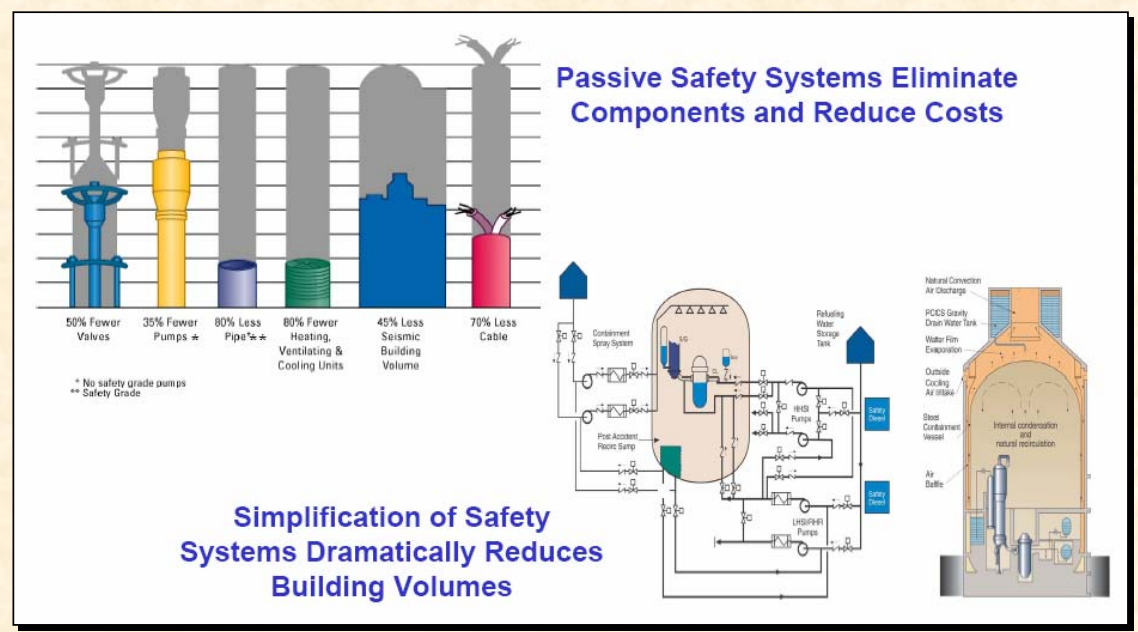

Source: Westinghouse

OAK RIDGE NATIONAL LABORATORY

U. S. DEPARTMENT OF ENERGY

UT-BATTELLE

\section{EPR Safety Features}

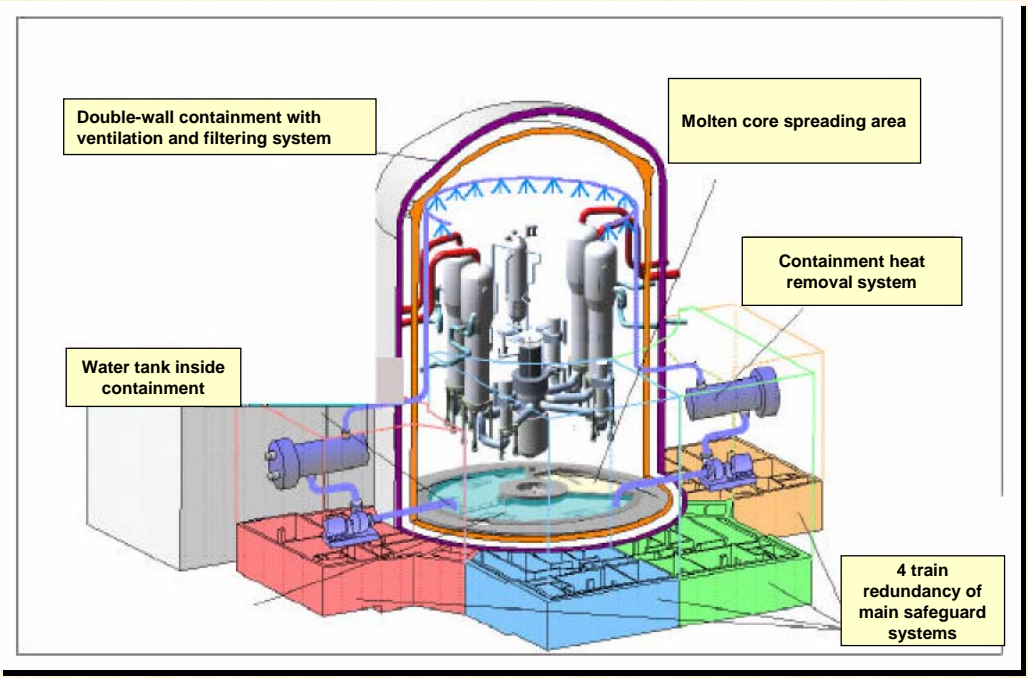

Source: Framatome ANP

OAK RIDGE NATIONAL LABORATORY

U. S. DEPARTMENT OF ENERGY

UT-BATTELLE 
Nuclear Reactor Technology Options

- Medium-to-long term (>2030) - GEN IV Reactors

- Enhanced safety

- Higher efficiency/temperature

- Coolants: Inert Gas, Liquid Metal, or Salt

- Brayton Cycle

- Hydrogen Production w/o $\mathrm{CO}_{2}$

- Closing of the fuel cycle

- Reduce waste

- Proliferation resistant fuels

\section{Generation IV Systems}

- Very-High-Temperature Reactor System

- Lead-Cooled Fast Reactor System

- Gas-Cooled Fast Reactor System

- Supercritical-Water-Cooled Reactor System

- Molten Salt Reactor System

- Sodium-Cooled Fast Reactor System

\section{Each system has R\&D challenges ahead -} none are certain of success 


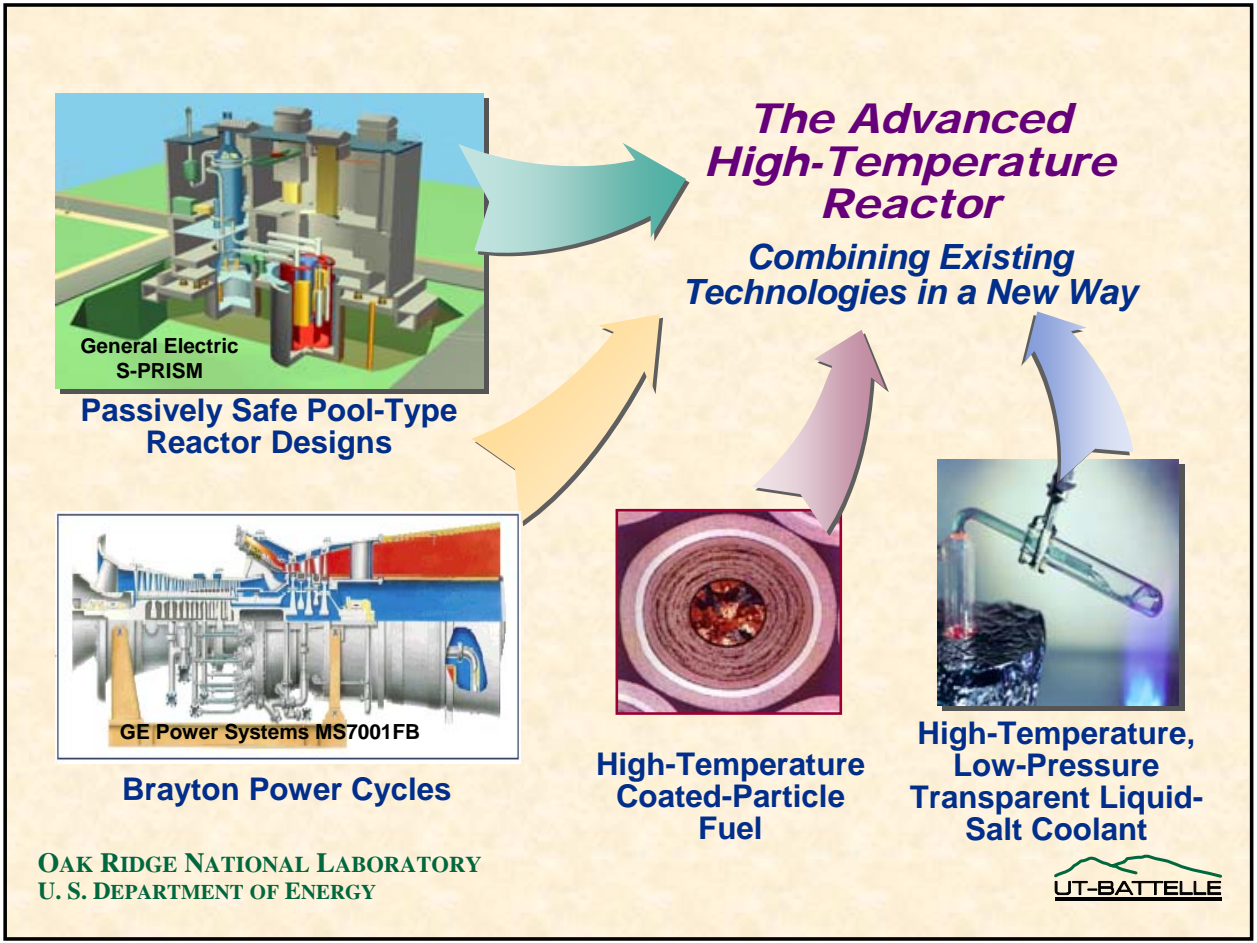

\section{There is New Interest in High-Temperature} Reactors Because of Brayton Technologies

- High-temperature heat for a utility is only useful if it can be converted to electricity

- Steam turbines (with a $550^{\circ} \mathrm{C}$ peak temperature) have been the only efficient, industrial method to convert heat to electricity

- Development of large efficient high-temperature Brayton cycles in the last decade makes hightemperature heat useful for electricity production

- Potential for economic high-temperature reactors

OAK RIDGE NATIONAL LABORATORY

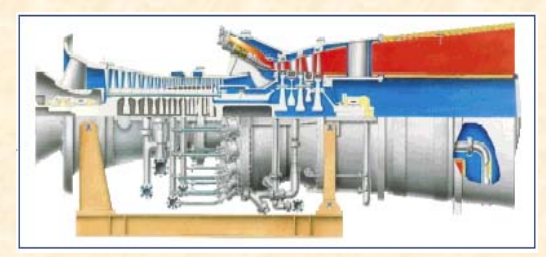

GE Power Systems

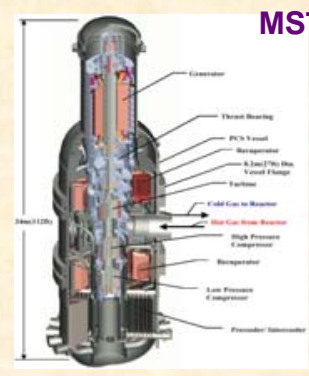

General Atomics GT-MHR Power Conversion Unit (Russian Design)

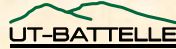




\section{Expansion of the Nuclear Energy Supply}

By 2050, with robust technology development:

- $50 \%$ of U.S. electricity production could be nuclear

- $25 \%$ of U.S. transportation could use hydrogen from nuclear energy

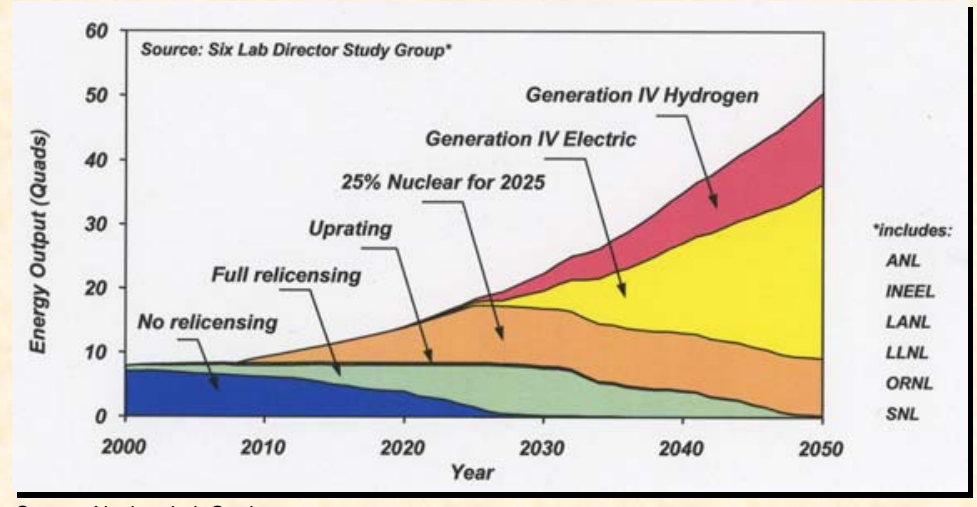

Source: Nuclear Lab Study
OAK RIDGE NATIONAL LABORATORY

U. S. DEPARTMENT OF ENERGY 



\section{UT-BATTELLE \\ ORELA Improvement Plan}

\section{Michael Dunn}

Nuclear Data Group Leader Nuclear Science \& Technology Division

ORELA Workshop

July 14-15, 2005

OAK RIDGE NATIONAL LABORATORY

U.S. Department of Energy

\section{ORELA Facility Improvement Needs}

Required for stable operation at any power level

Immediate improvement needs:

- Electron gun program revival

Staff expertise needed

- Vacuum, cooling upgrades

- Computer control of ORELA

- Thyratrons

Improvements to avoid single-point failures:

- Klystron

- Replacement target lift

- Remove positron target

*No spare, 6 month downtime in case of failure.

OAK RIDGE NATIONAL LABORATORY

U. S. DEPARTMENT OF ENERGY
Required for $50 \mathrm{~kW}$ operation

Desirable improvements:

- Add'l Thyratrons

- Add'I Klystrons

- Linac parts 


\section{ORELA Immediate Improvement Needs}

$>$ Electron Gun Program

- Guns manufactured in-house- “off-the-shelf” guns cannot be used

- Currently, ORELA does not have an inventory of parts needed to manufacture stable of guns

- Expect 1000 hours of operation/gun

- Need inventory of 3-4 spare guns

V Vacuum System

- New guard vacuum pumps

- Vac-ion pumps,

- Pump for target room

- Improved rough pump system with broad measurement capabilities

$>$ Cooling System

- Improved temperature control

- Increased heat removal capacity

- Better control of coolant flow to individual sections of the accelerator

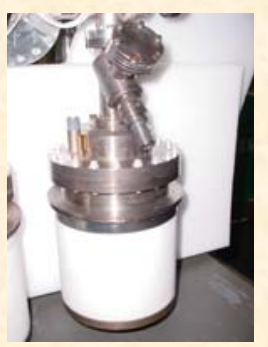

OAK RIDGE NATIONAL LABORATORY
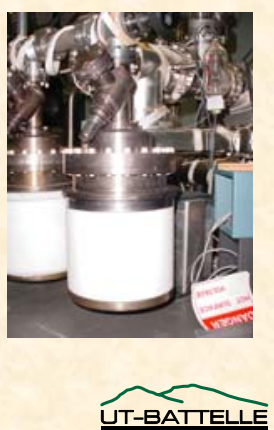

\section{ORELA Immediate Improvement Needs}

\section{Computer Control Improvements}

- Operator staff resources shared between Holifield Radioactive Ion Beam Facility (HRIBF) and ORELA

- Cannot provide 24-hour manned operation at both facilities

- Computer control with web capabilities needed for

- Monitoring

- Diagnosis

- Adjustment and resetting of systems
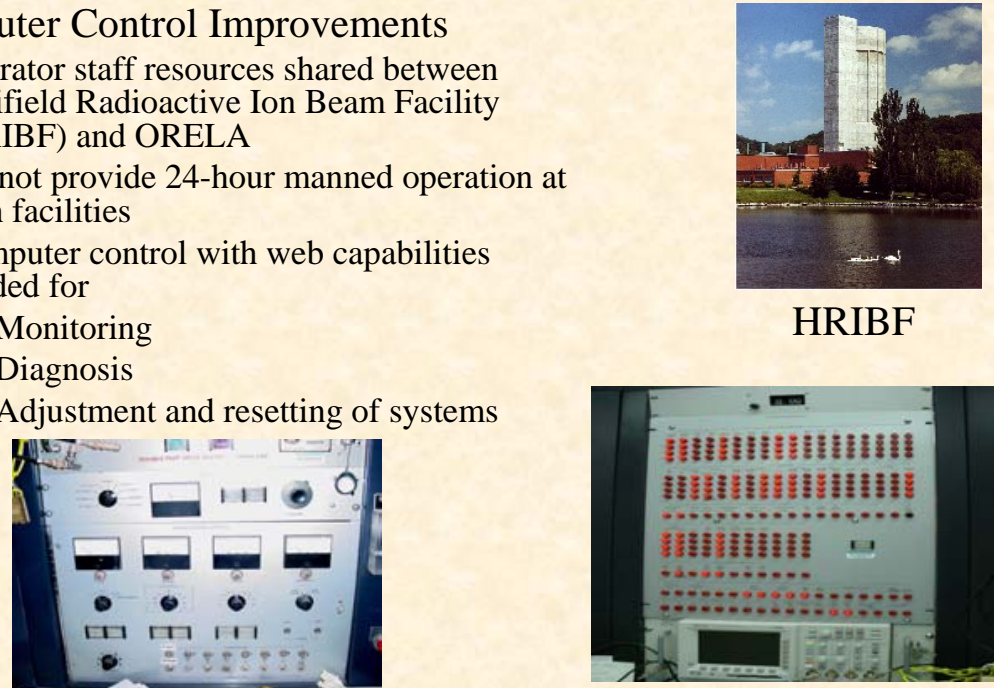

HRIBF

RF Control Panel

OAK RIDGE NATIONAL LABORATORY

U. S. DEPARTMENT OF ENERGY

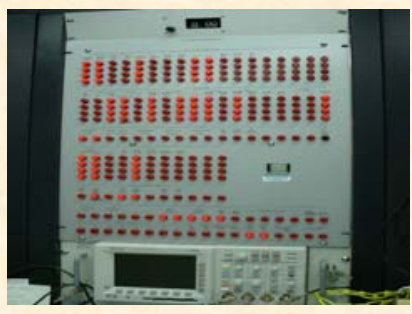

Interlock \& View Panel

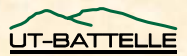




\section{ORELA Immediate Improvement Needs}

\section{Thyratrons and Klystrons}

- Have finite but unpredictable lifespan

- Need to replace in near-term to maintain reliable service

- All spare klystrons have been usedno inventory of spare klystrons

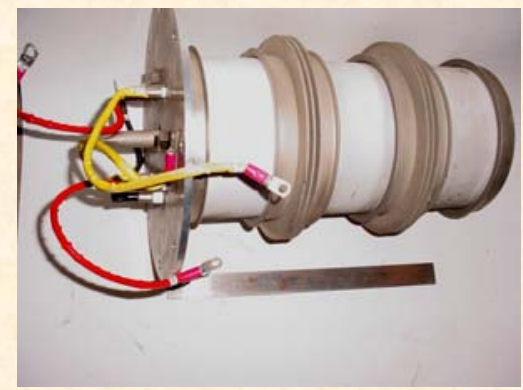

Thyratron

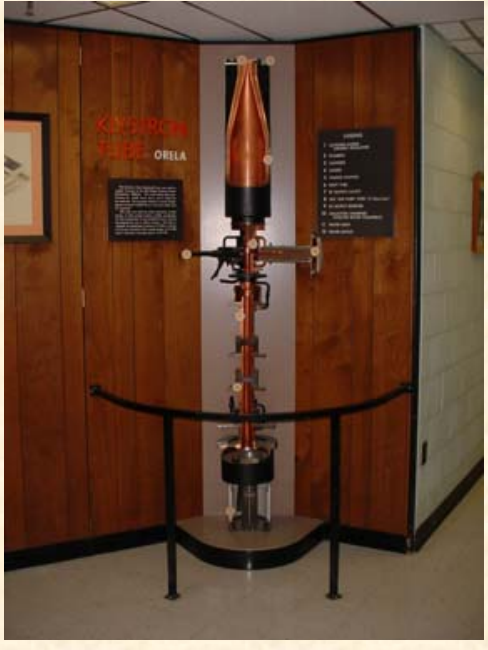

Klystron

OAK RIDGE NATIONAL LABORATORY

\section{ORELA Immediate Improvement Needs:} Improvements to Avoid Single-Point

\section{Failures}

\section{Target Lift}

- Lift has been in service for more than 30 years in very high radiation environment (ORELA Target Room)

- ORNL does not have spare target lift

- Target lift failure:

- minimum 6 month down time to acquire new lift and complete repairs

- Not off-the-shelf item-must fabricate new lift

- ORNL Physics Division planning measurements that will require access to target room and use of target lift system—reliability of target lift operation unknown

\section{Remove Positron Target}

- Located behind ORELA target-no longer needed

- Requires operational cooling systems because of beam interception

- Removal is needed to prevent shutdown maintenance of these systems that are located in the ORELA target room 


\section{ORELA Desirable Improvements}

ORELA full-power capacity: $50 \mathrm{~kW}$

Currently operated between a maximum of 7-15 kW

Benefit of higher power operation

- Smaller sample sizes for transmission and capture measurements-reduced sample costs

- Enables measurements on very rare isotopes as well as radioactive samples

- Position ORELA to respond to emerging measurement needs

Improvement Items

- Additional thyratrons and klystrons

- Need 4 additional klystrons over current configuration

- Higher consumption rate of klystrons and thyratrons due to higher power operation

- Linear accelerator parts

- Focusing coils and associate power supplies need to be replace or upgraded

- These components have deteriorated over the years

- Upgrades to modulators needed to accommodate additional thyratrons for $50 \mathrm{~kW}$ operation

OAK RIDGE NATIONAL LABORATORY

\section{ORELA Facility Improvement Plan}

Facility upgrades needed to increase ORELA reliability and maintain long-term operational readiness

- $\$ 1.5 \mathrm{M}$ crucial improvements

- $\$ 1.6 \mathrm{M}$ desirable improvements

$>$ Improvement Plan

- Immediate improvements: FY2006 \& FY2007

- Desirable improvements: FY2008-FY2010

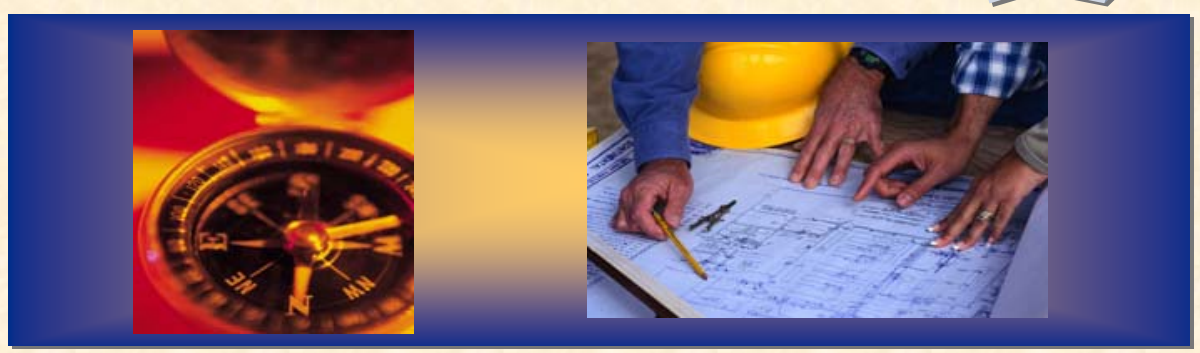

OAK RIDGE NATIONAL LABORATORY

U. S. DEPARTMENT OF ENERGY 


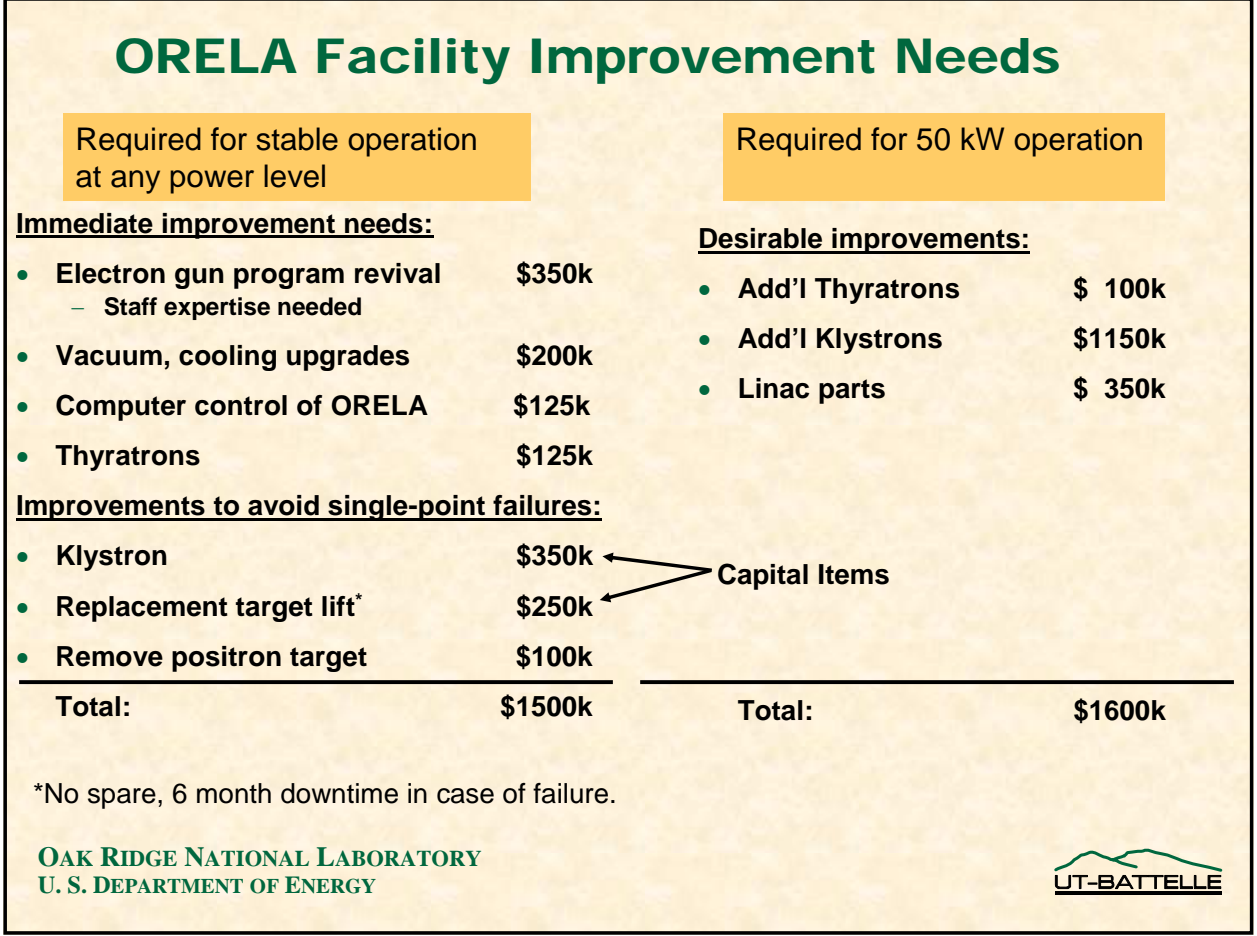



ORNL/TM-2005/272

\title{
INTERNAL DISTRIBUTION
}

\author{
1. Goran Arbanas \\ 5700, MS-6171 \\ 2. James R. Beene \\ 6000, MS-6358 \\ 3. Michelle V. Buchanan \\ $4500 N$, MS-6230
4. Herve Derrien 5700, MS-6171
5. Michael E. Dunn 5700, MS-6170
6. Edward C. Fox $4500 N$, MS-6248
7. Jess C. Gehin 5700, MS-6172
8. N. Maurice Greene 5700, MS-6171
9. Sherrell R. Greene 5700, MS-6162 \\ 10. Klaus H. Guber \\ 6010, MS-6356
11. John A. Harvey 6010, MS-6356
12. Calvin M. Hopper 5700, MS-6170
13. Daniel T. Ingersoll 5700, MS-6162
14. Jeffrey O. Johnson 5700, MS-6172
15. Sandra B. Kennedy 6010, MS-6372
16. Bernadette L. Kirk 5700, MS-6171
17. Paul E. Koehler 6010, MS-6356
18. Nancy M. Larson 5700, MS-6171
19. Luiz C. Leal 5700, MS-6171
20. Gordon E. Michaels 4500N, MS-6248
21. Cecil V. Parks 5700, MS-6170
22. Davis A. Reed 4500S, MS-6127
23. James B. Roberto 4500N, MS-6240
24. James E. Rushton 5700, MS-6152
25. Royce O. Sayer 5700, MS-6170
26. Brian A. Tatum 6000, MS-6368
27. Timothy E. Valentine 4500N, MS-6248
28. John C. Wagner 5700, MS-6170
29. Dorothea Wiarda 5700, MS-6171
30. R. Michael Westfall 5700, MS-6170
31. Mark L. Williams 5700, MS-6170
32. Glenn R. Young 6025, MS-6369
33. ORNL OTIC-RC 6011, MS-6283

\section{EXTERNAL DISTRIBUTION}

34. Richard Anderson, Los Alamos National Laboratory, P.O. Box 1663, Los Alamos, NM 87545

35. Joseph B. Briggs, Idaho National Laboratory, 2525 N. Fremont Avenue, Idaho Falls, ID 83415-3860

36. Lucia Cartegni, Universita Degli Studi Di Milano, via Illirico, 18, 20133 Milano, Italy

37. Linda Carter, 9702 Garden Row Drive, Sugar Land, TX 77478 USA

38. Mark B. Chadwick, Los Alamos National Laboratory, B283, PADNWP, LANL, Los Alamos, NM 87545

39. Yaron Danon, Rensselaer Polytechnic Institute, 110 8th Street, Troy, NY 12180

40. Jerrad Deason, Prairie View A\&M University, 803 Copeland, Lufkin, TX 76904

41. Frank Dietrich, Lawrence Livermore National Laboratory, MS L-414, PO Box 808, Livermore, CA 94550

42. Madeline Feltus, NE-20/Germantown Building, U.S. Department of Energy, 1000 Independence Ave., S.W., Washington, D.C. 20585-1290 
43. James R. Felty, Science Applications International Corporation, 2418 Dickerson Street, Arlington, VA 22207

44. Ivon E. Fergus, SP-44/Germantown Building, U.S. Department of Energy, 1000 Independence Ave., S.W., Washington, D.C. 20585-1290

45. Rodolfo M. Ferrer, Penn State University, 1013 South Allen Street, \#109, State College, PA 16801

46. Phil Finck, Argonne National Laboratory, 9700 S. Cass Avenue, Argonne, IL 60439

47. Edward K. Fujita, Argonne National Laboratory, 9700 S. Cass Avenue, Argonne, IL 60439

48. Adolf S. Garcia, Idaho National Laboratory, P.O. Box 1625, Idaho Falls, ID 83415

49. Marc A. Garland, University of South Carolina, 1136 Autumn Circle, Columbia, SC 29206-4986

50. Daniel F. Gill, Penn State University, 219 Waupelani Drive, Apt. 10, State College, PA 16801

51. Frank Goldner, NE-20/Germantown Building, U.S. Department of Energy, 1000 Independence Ave., S.W., Washington, D.C. 20585-1290

52. Reza R. Gouw, Bechtel Bettis, Inc, PO Box 79, West Mifflin, PA 15122

53. Robert C. Haight, Los Alamos National Laboratory, MS H855, Los Alamos, NM 87545

54. Ayman Hawari, Associate Professor and Director of Nuclear Reactor Program, Burlington Nuc Labs 2116, Box 7909, NCSU Campus, Raleigh, NC 27695

55. James C. Hawkins, SC-26.2/Germantown Building, U.S. Department of Energy, 1000 Independence Ave., S.W., Washington, D.C. 20585-1290

56. David P. Heinrichs, Lawrence Livermore National Laboratory, 7000 East Avenue, PO Box 808, L-198, Livermore, CA 94002

57. Eugene A. Henry, SC-26.1/Germantown Building, U.S. Department of Energy, 1000 Independence Ave., S.W., Washington, D.C. 20585-1290

58. Michal W. Herman, Brookhaven National Laboratory, Bldg. 197D, Upton, NY 11973

59. Jeffrey G. Hoole, Knolls Atomic Power Laboratory, PO Box 1072, Schenectady, NY 12301

60. Albert C. Kahler, III, Bechtel Bettis, Inc, PO Box 79, West Mifflin, PA 15122

61. Toshihiko Kawano, Los Alamos National Laboratory, MS B283, Theoretical Division, Los Alamos, NM 87545

62. Arthur K. Kerman, Massachusetts Institute of Technology, 5 Cambridge Center, $4^{\text {th }}$ Floor, Cambridge, MA 02142

63. Susan M. King, 90 Madrid Ct., Knoxville, TN 37923

64. Jesse J. Klingensmith, Penn State University, 1238 S. Pugh, State College, PA 16801

65. Dennis G. Kovar, SC-26/Germantown Building, U.S. Department of Energy, 1000 Independence Ave., S.W., Washington, D.C. 20585-1290

66. Sebastien Lemaire, Los Alamos National Laboratory, MS B243, Los Alamos, NM 87545

67. Kun Li, University of Illinois at Urbana - Champaign, 502 W. Main St., \#117, Urbana, IL 61801

68. Robert C. Little, Los Alamos National Laboratory, MS F663, PO Box 1663, Los Alamos, NM 87545

69. Henry A. Lovett, University of South Carolina, 835 Ohio Street, Columbia, SC 29201

70. Cecil Robert Lubitz, Knolls Atomic Power Laboratory, PO Box 1072, Schenectady, NY 12301-1072

71. Robert E. MacFarlane, Los Alamos National Laboratory, MS B243, Los Alamos, NM 87545 
72. Jerry N. McKamy, National Nuclear Security Administration, 1000 Independence Ave., SW, NA-117, Germantown Bldg., Washington, DC 20585-1290

73. Richard D. McKnight, Argonne National Laboratory, 9700 S. Cass Avenue, Argonne, IL 60439

74. Thomas P. McLaughlin, Los Alamos National Laboratory, Mail Stop F691, PO Box 1663, Los Alamos, NM 87544-0600

75. Gary E. Mitchell, North Carolina State University, Dept. of Physics, Box 8202, Raleigh, NC 27695

76. James Morman, Argonne National Laboratory, 9700 S. Case Avenue, Argonne, IL 60439

77. Mohammed G. Mustafa, Lawrence Livermore National Laboratory, 7000 East Avenue, Livermore, CA 94550

78. David W. Nigg, Idaho National Laboratory, PO Box 1625, Idaho Falls, ID 83415

79. Pavel Oblozinsky, Brookhaven National Laboratory, PO Box 5000, Upton, NY 11973

80. Megan Pritchard, 509A East $24^{\text {th }}$ Street, Bryan, TX 77803

81. Thomas Reilly, 1800 Robinson Drive, North Augusta, SC 29841

82. Rahn H. Ross, Washington Safety Management Solutions, 2131 Centennial Drive, Aiken, SC 29803

83. Jehanne E. Simon-Gillo, SC-26.2/Germantown Building, U.S. Department of Energy, 1000 Independence Ave., S.W., Washington, D.C. 20585-1290

84. Rachel N. Slaybaugh, Penn State University, 305 Ghaner Drive, State College, PA 16803

85. Thomas M. Sutton, Knolls Atomic Power Laboratory, PO Box 1072, MS-112, Schenectady, NY 12301-1072

86. Hans Toffer, FFS, 1200 Jadwin Avenue, MSIN A0-26, P.O. Box 1050, Richland, WA 99352-1050

87. Alexander Y. Urashkin, Texas A\&M University, 4110 College Main, Apt. 46, Bryan, TX 77801

88. Susan N. Williams, 2706 Parside, McKinney, TX 75070

89. Robert E. Wilson, U.S. Department of Energy, 1000 Independence Ave., S.W., EM-22, Washington, DC 20585

90. Eric L. Wright, Faculty and Student Team Program (FaST), 13411 Blackstock Lane, Houston, TX 77083

91. Richard Q. Wright, 104 W. Newcomb Road, Oak Ridge, TN 37831 